\title{
DESIGN AND OPERATION OF A GEOPRESSURED-GEOTHERMAL HYBRID CYCLE POWER PLANT
}

FINAL REPORT

FEBRUARY 1991

PREPARED FOR

EATON OPERATING COMPANY, INC.

AND

UNITED STATES DEPARTMENT OF ENERGY

UNDER

DOE CONTRACT NO. DE-AC07-85ID12578

BY

THE BEN HOLT CO.

PASADENA, CALIFORNIA 


\section{DISCLAIMER}

This report was prepared as an account of work sponsored by an agency of the United States Government. Neither the United States Government nor any agency Thereof, nor any of their employees, makes any warranty, express or implied, or assumes any legal liability or responsibility for the accuracy, completeness, or usefulness of any information, apparatus, product, or process disclosed, or represents that its use would not infringe privately owned rights. Reference herein to any specific commercial product, process, or service by trade name, trademark, manufacturer, or otherwise does not necessarily constitute or imply its endorsement, recommendation, or favoring by the United States Government or any agency thereof. The views and opinions of authors expressed herein do not necessarily state or reflect those of the United States Government or any agency thereof. 


\section{DISCLAIMER}

Portions of this document may be illegible in electronic image products. Images are produced from the best available original document. 


\section{RECEIVED JUN 211993 OSTI}

\section{APPENDIX B-1 \\ PLEASANT BAYOU SITE}

THE BEN HOLT CO.

FINAL REPORT

OF

THE HYBRID POWER SYSTEM (HPS)

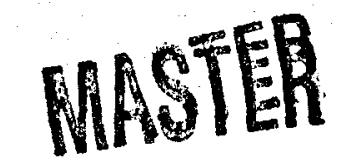

DISTFEITLON OF THU DOOUMENT E UNLIMITED 
DESIGN AND OPERATION OF A GEOPRESSURED-GEOTHERMAL HYBRID CYCLE POWER PLANT

\author{
FINAL REPORT \\ VoluE:!! \\ February 1991 \\ by \\ Richard G. Campbell \\ and \\ Mai M. Hattar \\ for \\ EATON OPERATING COMPANY, INC. \\ AND
}

UNITED STATES DEPARTMENT OF ENERGY

The Ben Holt Co.

Pasadena, CA.

DOE Contract No. DE-AC07-85ID12578

Holt Project No. 30008 


\section{INDEX}

A Process Flow Diagram

B Piping and Instrumentation Diagram

C New Equipment Specifications

D Main Single Line Diagram

E Shutdown \& Start-Up Procedures

F Data Sheets for Tests

G Plant Outages

- H Detailed Process Equations

I Computer Program and Sample Output

J Chemical Analysis and Scanning Electron

Microscopy Results

K Management Report Data Sheets

January 5, 1990 - May 29, 1990 
APPENDIX A

PROCESS FLOW DIAGRAM

THE BEN HOLT CO. 


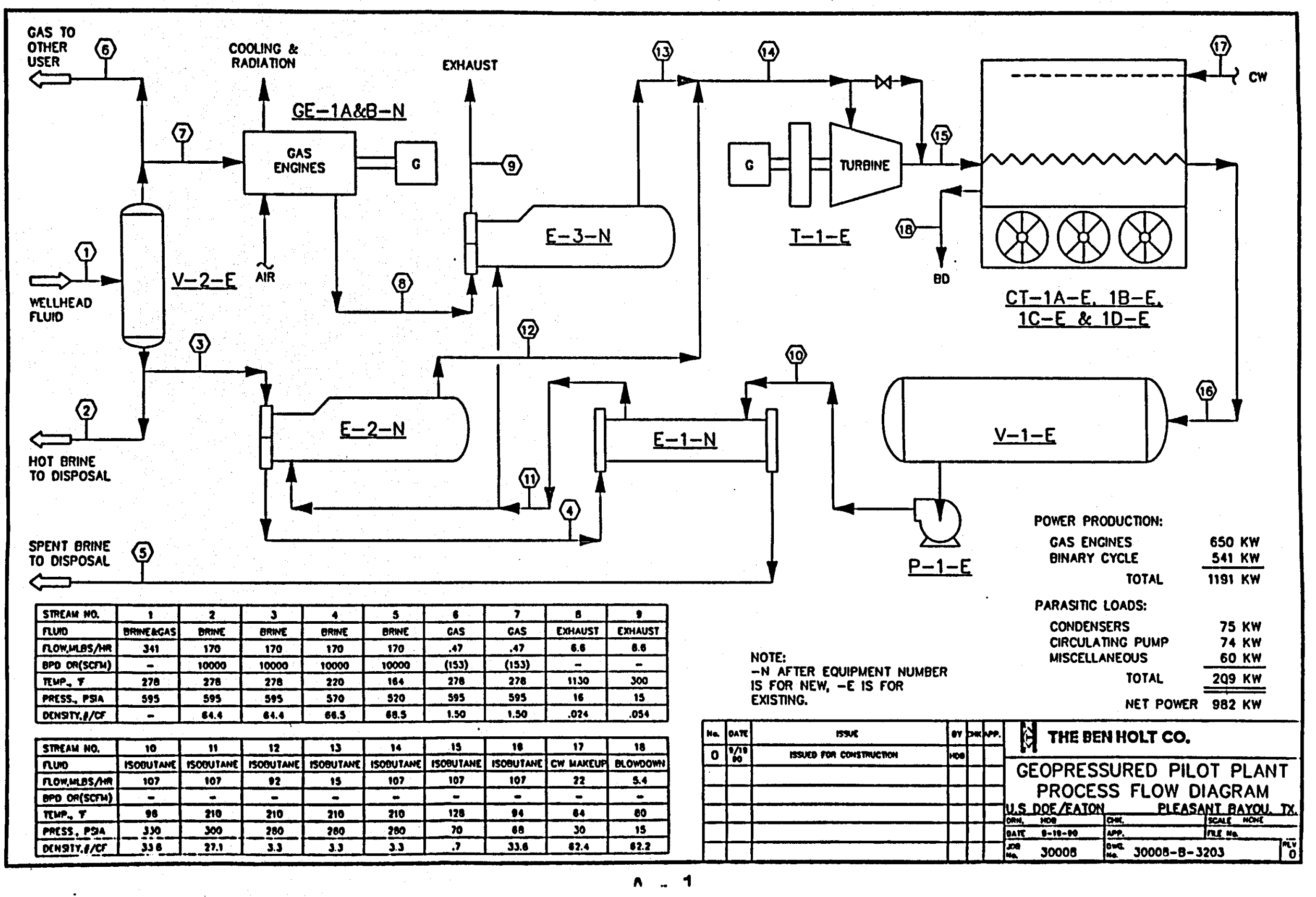




\section{APPENDIX B}

PIPING AND INSTRUMENTATION DIAGRAM

THE BEN HOLTCO. 


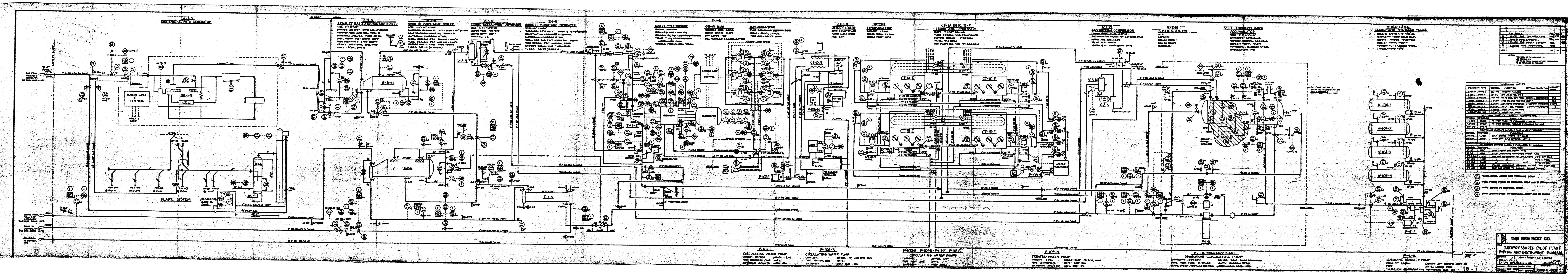




\section{APPENDIX C \\ SPECIFICATIONS OF NEW EQUIPMENT}

INDEX

C. 1 Geothermal Brine to Isobutane Preheat Heat Exchanger, E-1-N

C- 2 Geothermal Brine to Boiling Isobutane Heat Exchanger, E-2-N

C- 3 Exhaust Gas to Boiling Isobutane Heat Exchanger, E-3-N

C- 4 Isobutane Separator, V-2-N

C- 5 Utility Cooler

C. 6 Gas-Freeing Compressor

C. 7 Cooling Water Controller 


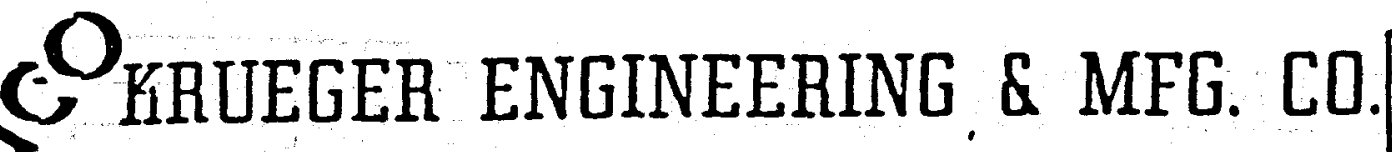
EXCHANGER SPECIFICATION SHEET

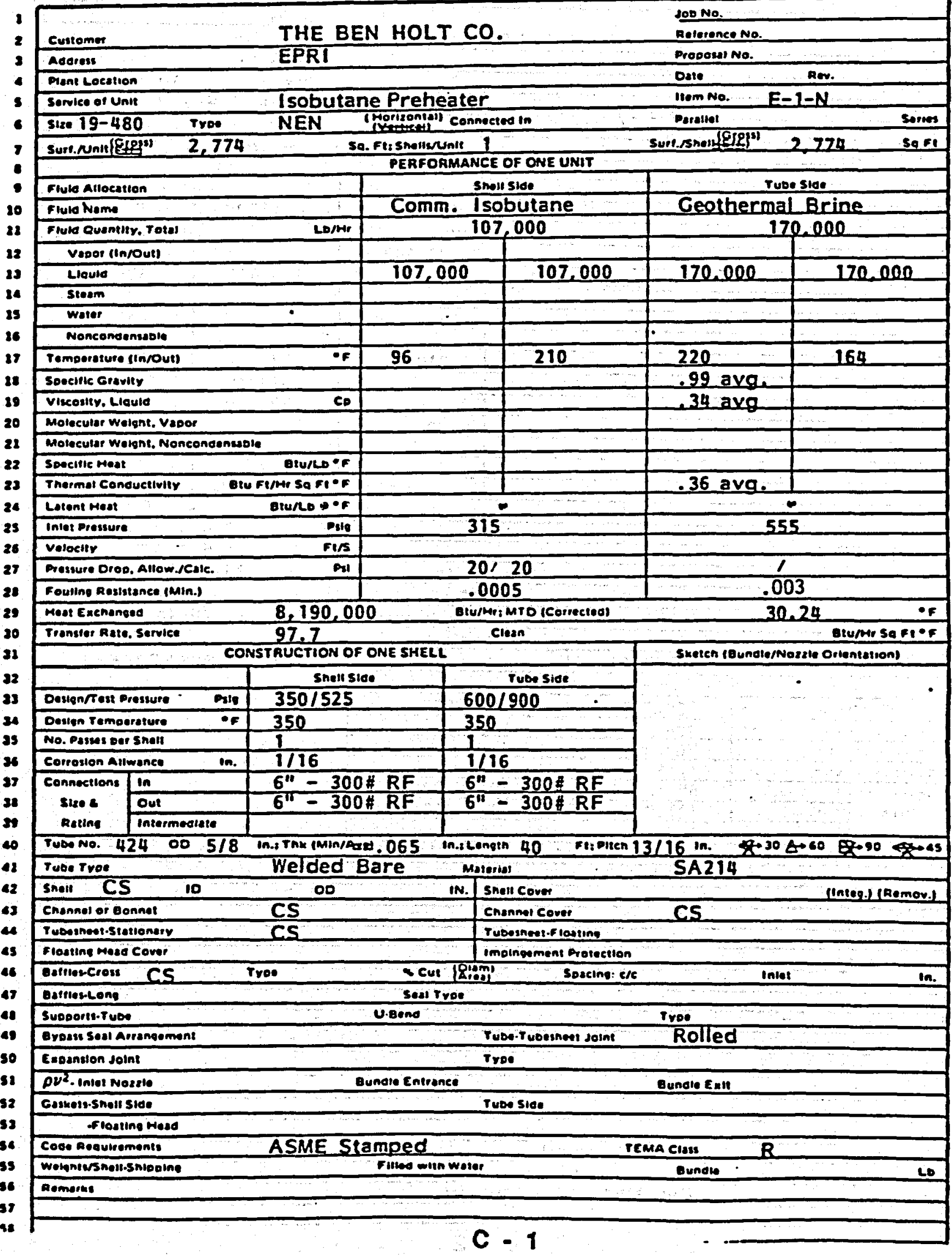




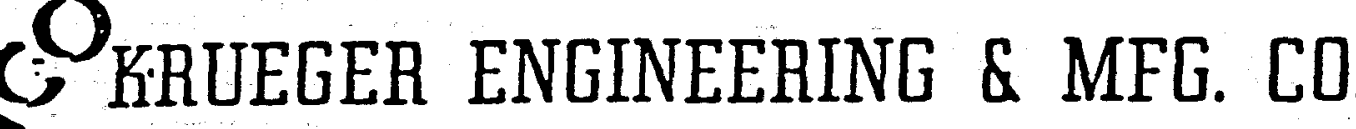

\section{EXCHANGER SPECIFICATION SHEET}

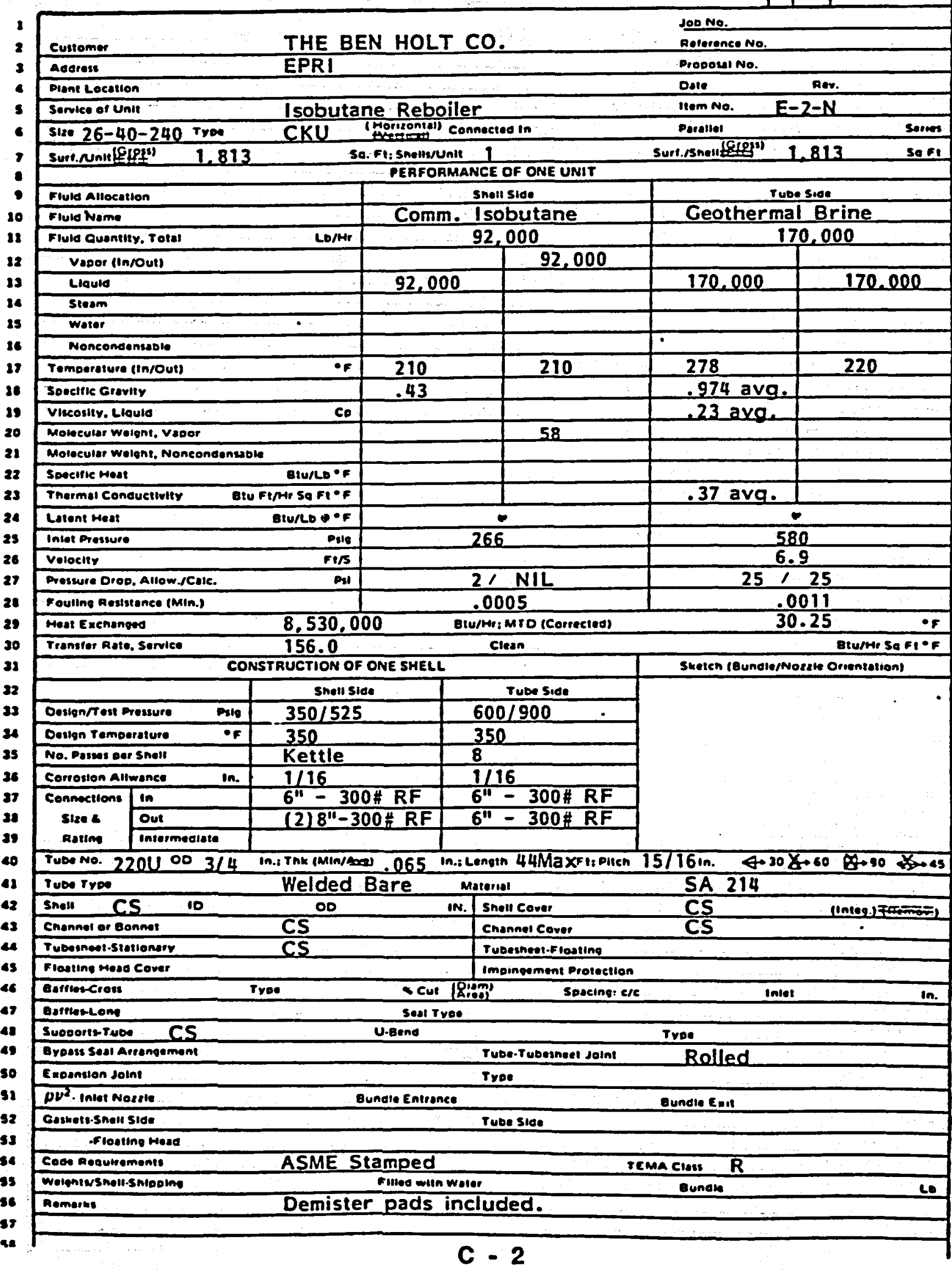




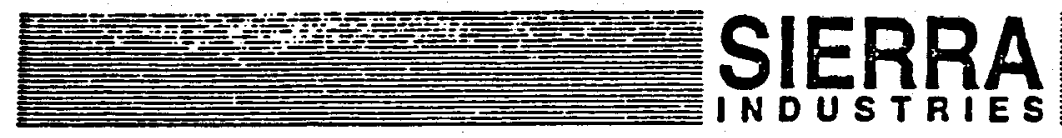

\section{HEAT EXCHANGER SPECIFICATIONS}

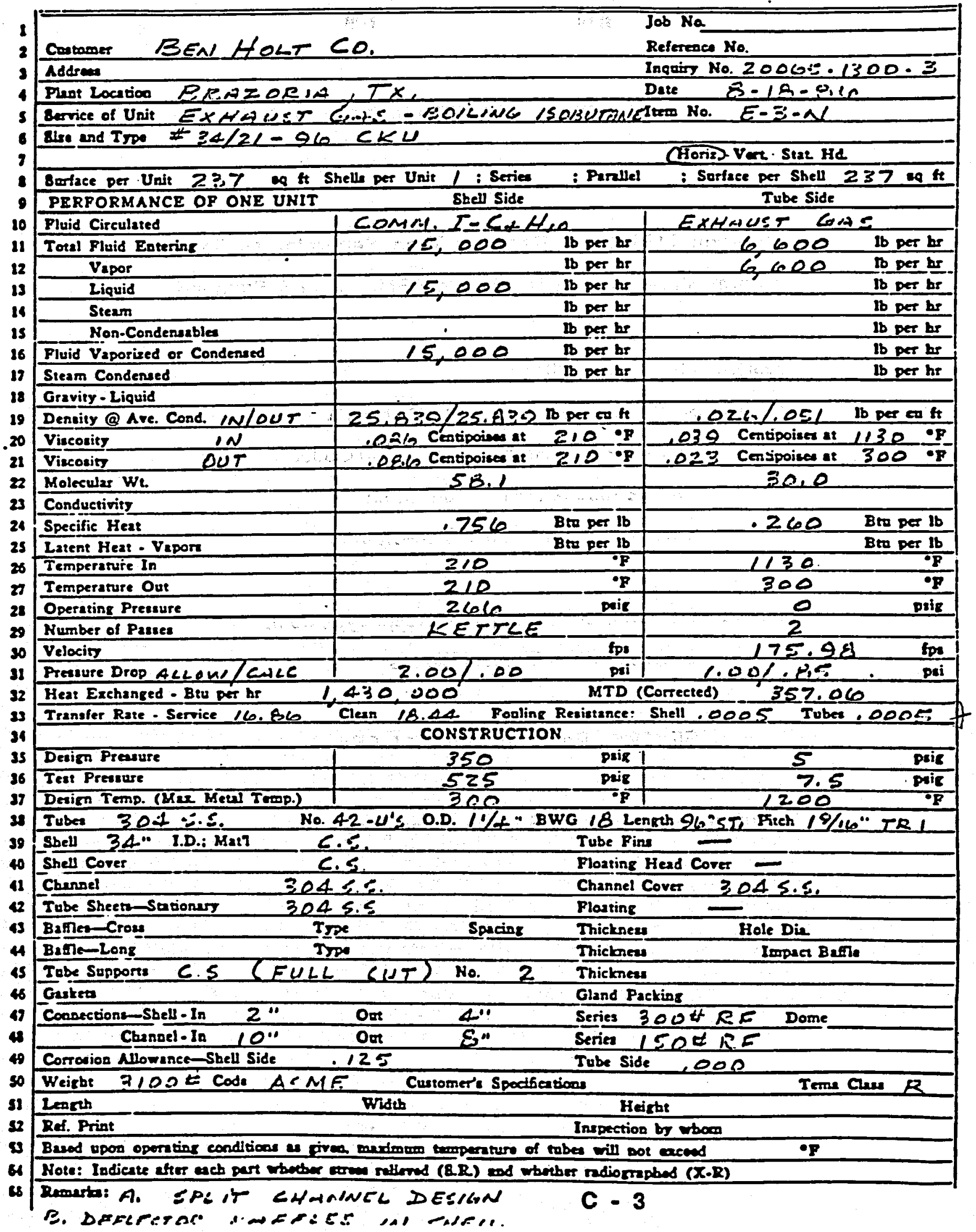




\section{ISOBUTANE SEPARATOR, V-2-N}

Anderson LUS-8-300 Receiver Type Separator similar to Form Sheet Type LRU. Built to ASME Code \& Stamped.

Design: 300 PSIG $300^{\circ} \mathrm{F}$

Physical Size: $16^{n}$ O.D. X 59" Overall Height

Nozzles: Two 8"-300\# RF Side Inlet \& Top Outlet

One 2"-3000\# NPT Bottom Drain

One 1 1/2"-3000\# NPT Side-Drain

Material: Carbon Steel Throughout Except Type 304L

Stainless Steel Element Blades

Eff. 99\%, Pressure Drop 1.14 PSI

Manufacturer: Anderson Separator Company 


\section{UTILITY COOLER}

Baltimore Aircoil Closed Circuit Industrial Fluid Cooler Model VX1 36-3. Capacity to cool 275 GPM.

Furnished with one $15 \mathrm{HP}, 230 / 460$ volt, 3 phase, 60 cycle fan motor.

Also furnished with one $1 \mathrm{HP}, 230 / 460$ volt, 3 phase, 60 cycle spray water pump.

Approximate shipping weight of the unit is $\mathbf{5 1 4 0}$ lbs. The unit ships in two pieces, with the heaviest section being $3720 \mathrm{lbs}$.

$$
\text { C }-5
$$




\section{GAS-FREEING COMPRESSOR}

One Corken model D390-109A two stage, base mounted, non lubricated gas compressor. The compressor is mounted on a base with the following equipment:

- 7.5 HP, 460 VAC, TEFC electric motor.

- V-belt drive with enclosed belt guard.

- Air cooled interstage cooler.

- Liquid trap on suction with explosion-proof high liquid level switch.

- Pressure Gauges. 


\section{APPLICATIONS}

Custom prefabricated package including a centrifugal separator. The separator operates on a bypass to remove suspended particles from the sample flow and the cooling water - heat exchanger loop. A motorized ball valve blows down the separator with the normal cooling water blowdown. The effectiveness of the separator depends on the suspended particle size and other factors. One installation in a steel plant effectively removed heavy sludge from the cooling water.

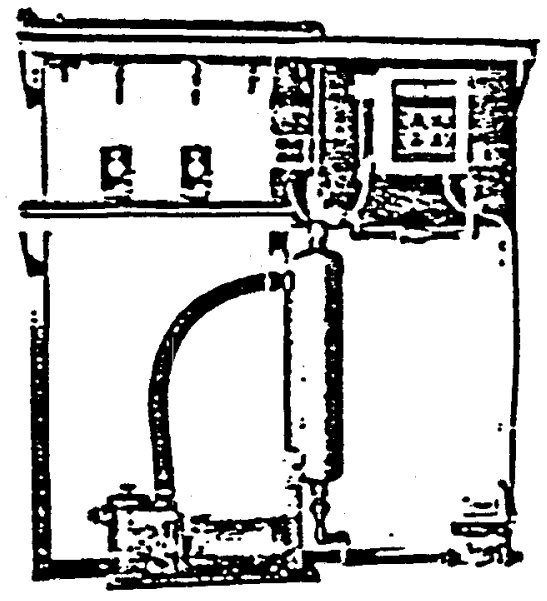

Another custom system included two Model 6100 modular controllers on a single floor supported wall rack. This saved floor space and the requirement for two sets of chemical drums. Two separate recirculation water loops could not be combined and space was limited. The custom prefabricated system reduced installation and chemical handling costs.

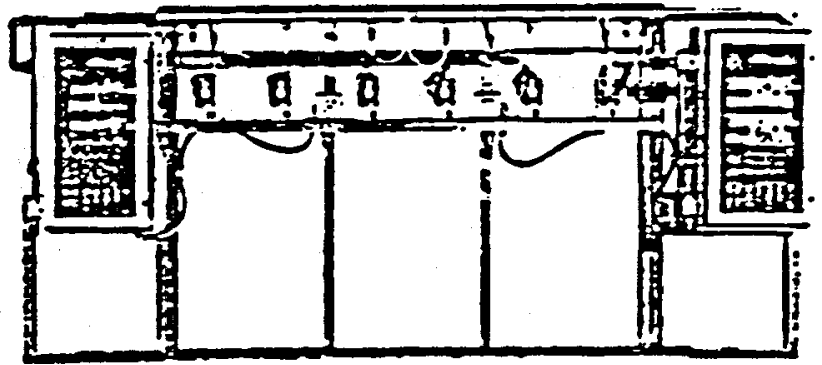

\section{GENERAL SPECIFICATIONS}

\section{MODEL $21-412$}

Dimensions

Material

Enclosure

Shipping weight

Plumbing

Acid firting

Dimensions

Material

Finish

Plumbing

Acid litting $30^{\circ} \mathrm{H} \times 48^{\circ} \mathrm{W} \times 12^{\circ} \mathrm{D}$

Epoxy coated steel

Window door with key lock

\%:" Schedule 80 PVC

\%"* FNPT injector. CPVC

\section{MODELS 9301 - 9305}

9301

9302 and 9303

$64^{\circ} \mathrm{H} \times 84^{\circ} \mathrm{W} \times 15^{\circ} \mathrm{D}$

9304

9305

$64^{\circ "} \mathrm{H} \times 112^{\circ} \mathrm{W} \times 15^{\circ} \mathrm{D}$

$64^{\circ} \mathrm{H} \times 142^{\circ} \mathrm{W} \times 15^{\circ} \mathrm{D}$

$1 \%$ " square welded tube

Epoxy coated

$y_{4}$ " Schedule 80 PVC

$\%$ \%NPT injector. CPVC
MODEL 25-412

$31^{\circ \circ} \mathrm{H} \times 38^{\circ} \mathrm{W} \times 12^{\circ} \mathrm{O}$

UV stabilized polyethylene

316 SS hardware

Sliding plexiglass safety panels

$y_{2}$ " Schedule 80 PVC

\\%" FNPT injector. CPVC

MODELS 9310 - 9312

$9310.30^{\circ} \mathrm{H} \times 68^{\circ} \mathrm{W} \times 12^{\circ \circ} \mathrm{D}$

$9311.30^{\circ} \mathrm{H} \times 68^{\circ} \mathrm{W} \times 12^{\circ} \mathrm{O}$

9312, $30^{\circ} \mathrm{H} \times 68^{\circ} \mathrm{W} \times 12^{\circ} \mathrm{D}$

12 gauge steel

Epoxy coated

\%." Schedule 80 PVC

y.* FNPT injector. CPVC 
APPENDIX D

MAIN SINGLE LINE DIAGRAM

THE BEN HOLT CO. 


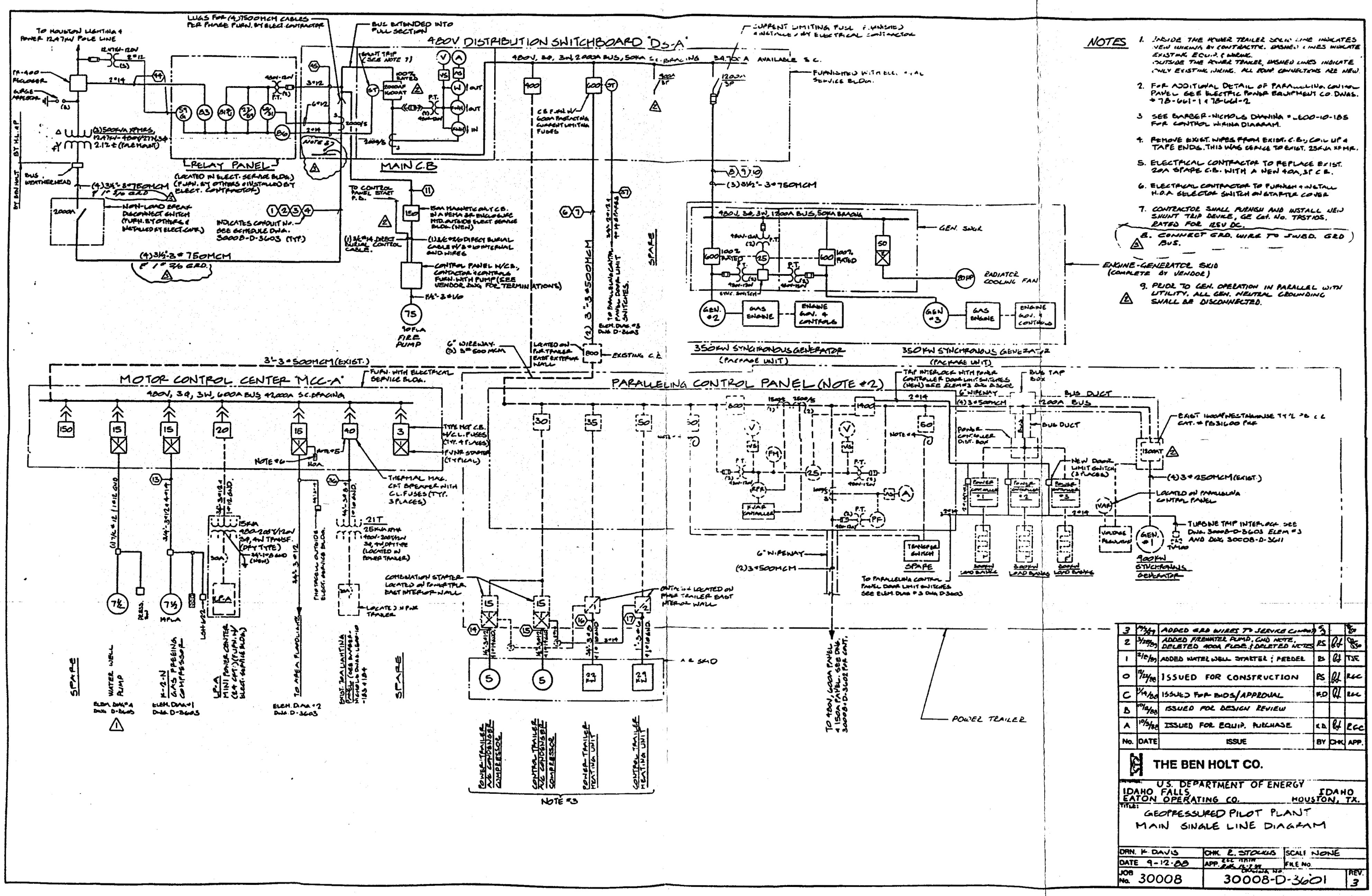

D - 1 


\section{APPENDIX E}

\section{SHUTDOWN AND START-UP PROCEDURES}




\section{PLEASANT BAYOU HPS}

\section{SHUTDOWN AND STARTUP CHECKLIST PROCEDURES}

\section{TABLE OF CONTENTS}

Paragraph

Page

1.0 SHUTDOWN AFTER TURBINE TRIP.................... 2

2.0 STARTUP AFTER TURBINE TRIP................... 4

3.0 SHUTDOWN AFTER ELECTRICAL TRIP................. 6

4.0 STARTUP AFTER ELECTRICAL TRIP ................ 


\subsection{SHUTDOWN AFTER TURBINE TRIP}

1.1. Silence only, Annunciator No. 1, left side alarm panel.

Acknowledge only, Annunciator No. 2, right side alarm panel if it alarms.

1.2. Monitor vibration monitor as turbine rolls down.

Step 1.3. should be in progress while monitoring vibrations.

1.3. Reduce brine rate until $\mathrm{Pl}-116$ is between 270-280 psig. This should be $85-90 \mathrm{M} \mathrm{lbs} / \mathrm{hr}$ if both Gas Engines are on line to $E-3-N$. Monitor E-3-N level while reducing brine rate.

1.4. Switch Level 1 Shutdown to OFF.

1.5. Switch Vanes PY-105 (P1C-109 Turbine Inlet Governor) to Manual and close to $0 \%$.

1.6. Switch Bypass $\mathrm{CV}-100$ (P1C-103 Turbine Inlet Bypass) to Manual and open to $100 \%$.

1.7. Shut off Lube 0il cooling water outlet block valve HV-661 (Lower valve).

1.8. Switch Voltage Regulator to Manual.

1.9. Investigate cause of trip, $\log$ results, and report to supervisor the following items:

1.9.1. Generator and Utility breaker positions.

1.9.2. Lube 0il temperatures and pressures.

1.9.3. Vane position at turbine.

1.9.4. Trip valve position at turbine.

1.9.5. Gas Engines conditions.

1.9.6. Electrical Building breaker positions.

1.9.7. Annunciator alarm conditions.

1.9.8. Vibration monitor alert and danger conditions, and vibration during roll down if detected. 
1.10. Lube Oil circulation can be maintained if start-up is anticipated within 2 hours. If Lube oil circulation is to be shut down, ensure turbine has come to a complete stop.

1.11. Condenser fans and Utility Cooling system can remain running if start-up is anticipated within a few hours.

If turbine is to remain down, shut of $f$ condenser fans to maintain PI-104 ( $V-I-E$ contents) between 60-70 psig. Shut off utility cooler fan, circulating pumps $(P-106$ and $P-107)$ and water conditioner $p H$ and conductivity controllers.

1.12. If the isobutane flow $(F /-102)$ does not stabilize causing $\mathrm{CV}-117$ (FIC-102 isobutane flow from $P-1-E$ ) to open, then put on Manual and open to $100 \%$. 


\subsection{START-UP AFTER TURBINE TRIP}

2.1. Prior to start-up the cause of turbine trip should have been determined and remedial action taken.

2.2. Turn on Utility Cooler pumps (P-106 and $P-107)$, fan, and put water conditioner on control.

2.3. Turn on all condenser fans.

2.4. Turn on one Lube $0 i 1$ pump and put the other one on standby.

2.5. Ensure that Voltage Regulator is on Manual.

2.6. Ensure that turbane vanes are closed at the turbine and turbine case is warm. Drain liquid from turbine to warm if required.

2.7. Ensure nitrogen pressure to Emergency Lube Oil Accumulator bladder is properly set and Air to Gearbox at 2 psig.

2.8. Ensure that cooling water is circulating properly to load banks.

2.9. Reset annunciator alarm panels, shutdown alarm (yellow button) and vibration monitor.

2.10. Adjust brine rate to maintain PI-116 at 275 psig.

2.11. Switch bypass CV-100 (PIC-103 Turbine Inlet Bypass) to Automatic. Set point should be at or near actual pressure (about $7 \overline{0} \%$ ) so that bypass controller just begins to indicate closing. Immediately proceed to next step so that controller does not go to (or near) $0 \%$ causing overpressuring of system when Level 1 shutdown is enabled. Remember that bypass control valve only moves to controller position after enabling Level 1 shutdown.

Note:

If next step is delayed or another start-up attempt is required, switch $\mathrm{CV}-100$ to manual and open to $100 \%$, then repeat step 2.11 .

2.12. Switch Level 1 Shutdown to Enable.

2.13. Push Level 1 Shutdown "push button". This will enable Bypass. Control Valve and open trip valve.

Note:

Turbine Speed (RPM) should bounce to a few hundred and return to zero. If not switch Level 1 Shutdown to OFF.

2.14. Begin opening vanes manually after Turbine Speed has returned to zero. Increase speed to about 22,000 PRM within 45 seconds. 
2.15. Continue opening vanes at a slightly slower rate until governor controls speed at around 25,000 RPM.

2.16. Continue opening vanes increasing load on Generator until vibrations stabilize at a point below $3.0 \mathrm{mils}$ (normally around 300 $\mathrm{KW}$ on $\mathrm{J} 1-100)$.

NEVER RE-ROLL TURBINE UNTIL SPEED HAS RETURNED TO ZERO

2.17. Adjust vanes to maintain PI-116 around 275 psig, then switch vanes controller PY-105 to Automatic with a set point around $67 \%$.

Ensure pressure and vibrations have stabilized.

2.18. Open manual block valve HV-661 for cooling water to Lube Oil Cooler.

2.19. Parallel Turbine-Generator to HL \& P.

2.19.1. Switch Synchronizing knob to pos. 1 (Gen. to Util.)

2.19.2. Ajust voltage to match HL \& .

2.19.3. Adjust frequency to slowly rotate sync scope.

2.19.4: Close generator breaker when scope at 12:00.

2.19.5. Record synchronizing time.

2.20. Switch Voltage Regulator to Automatic, and ensure controlling at around 1.0 power factor.

2.21. Monitor Lube $0_{i l}$ temperatures and adjust as required.

2.22. Check Gas Engines power factors and adjust as required.

2.23. Increase brine rate and load Generator per orders.

2.24. Monitor vane position while loading generator, keeping $T-1-E$ inlet pressure $(P I-107)$ between 260 and 275 psig.

2.25. When vanes are $100 \%$ open (or per orders) adjust brine rate to. maintain PI- 107 between 260 and 270 psig.

2.26. Monitor and record vibrations as you load generator.

2.27. Manually close $\mathrm{CV}-117$ (FIC-102 Isobutane Flow from $P-1-E$ ) and switch to Automatic with a set point of $30 \%$.

2.28. Check Turbine area for isobutane leaks.

$$
\mathbf{E}-\mathbf{5}
$$


7.n. SHUTDOWN AFTER ELECTRICAL TRIP

3.1. Silence Annunciator alarm panels.

3.2. Switch Level 1 Shutdown to OFF.

3.3. Manual1y close $\mathrm{CV}-632$ (Exhaust Gas to E-3-N) to $0 \%$

3.4. Switch vanes PY-105 (PIC-109 Turbine Inlet Governor) to Manual and close to $0 \%$.

3.5. Switch Bypass CV-100 (PIC-103 Turbine Inlet Bypass) to Manual and open to $100 \%$.

3.6. Switch CV-104 (LIC-604 Isobutane Level E-3-N) to Manual and close to $0 \%$.

3.7. Switch CV-116 (FIC-601 Brine Flow Out) to Manual and close to $0 \%$. Needle Valve on half inch tubing bypass to be closed if extended shutdown anticipated.

3.8. Switch CV-117 (FIC-102 Isobutane Flow P-1-E) to Manual and open to $100 \%$.

3.9. Shut off Lube Oil Cooling Water outlet block valve HV-66l (lower valve).

3.10. Switch Voltage Regulator to Manual.

3.11. Investigate cause of trip, $\log$ results and report to supervisor the following:

3.11.1. Turbine Generator breaker position.

3.11.2. Turbine Utility breaker position.

3.11.3. Power Trailer outside breaker position.

3.11.4. Gas Engine Generator breakers positions.

3.11.5. Electrical Building breakers positions.

- Main Breaker

- Power Trailer Switchgear Breaker

- MCC-A Breaker

- Gas Engines Breaker

3.11.6. Main Breaker Fault Trip Annunciator positions.

3.11.7. 86 Lockout relay position.

$$
E-6
$$


3.11.8. Flags on any relays on Westinghouse Panel.

3.11.9. Line Voltage from HL\&P.

3.12. If no line voltage from HL \& P, call to have them reset their Reclosure Switch. Our Main Breaker must be open (the OFF position) prior to HL \& P resetting their Reclosure Switch.

NEVER HAVE HL \& P CLOSE THEIR RECLOSURE WHEN OUR MAIN BREAKER IS CLOSED (ON POSITION).

NEVER CLOSE OUR MAIN BREAKER WHEN ANY PART OF PLANT IS IN OPERATION. 


\subsection{START-UP AFTFR ELFCTRICAL TRIP}

NEVER HAVE HL \& P CLOSE THEIR RECLOSURE WHEN OUR MAIN BREAKER IS CLOSED (ON POSITION).

NEVER CLOSE OUR MAIN BREAKER WHEN ANY PART OF PLANT IS IN OPERATION.

4.1. Prior to start-up the cause of electrical trip should have been determined and remedial action taken.

4.2. Shut off all fans and pump motor switches in power trailer (including Lube Oil pumps) with the exception of Instrument Air. Switch Water Conditioners controllers to off and unplug chlorine pump.

4.3. HL \& P should have reset their reclosure switch if necessary. Ensure our Main Breaker is open.

4.4. Ensure that Turbine Generator, Gas Engine No. 1 and No. 2 Generator breakers are open.

4.5. Reset relay flags.

4.6. Close any tripped breakers except Main Breaker, three Generator Breakers or Power Trailer Utility Breakers.

4.7. Reset Main Breaker fault buttons.

4.8. Close Main Breaker.

4.9. Close Power Trailer Utility Breaker,

4.10. Ensure Instrument Air Compressor operating properly.

4.11. When Instrument Air Pressure builds up enough pressure (about 40 psig) to open CV-612 (Gas Engine Gas Valve), start up and parallel Gas Engines.

4.12. When Instrument Air pressure builds up to normal (80-100 psig), check Isobutane Loop for normal line up.

4.13. Reset FV-636 and ensure CV-123 is open $100 \%$.

4.14. Open $\mathrm{CV}-130$ for isobutane heat up.

4.15. Start all condenser pumps and single fans, and put water conditioner on control. Plug in chlorine pump.

$$
E-8
$$


4.16. Start-up Isobutane pump until level re-established in $E-2-N$. E-3-N Level can also be re-established at this time.

4.17. Shut down Isobutane pump.

4.18. Re-open CV-116 (Brine Control Valve) bypass needle valve if closed. Continue this slow heat-up until $\mathrm{E}-2 \mathrm{~N}$ has warmed up. Re-establish E-2-N level with isobutane pump as required.

4.19. E-3-N can also be slowly heated up at this time. CV-104 (LIC-604 Isobutane Level E-3-N) should be on Automatic control.

4.20. When $E-2-N$ heat-up is re-established, start up isobutane pump, slowly open CV-116 (Brine Control Valve) and slowly establish normal turbine bypass conditions. When brine flow rate is near normal rates, line up set point to process flow and switch to Automatic.

4.21. When isobutane is at normal temperatures, close cV-130.

4.22. Check isobutane system for isobutane leaks.

4.23. Check V-I-E, E-I-N and E-2-N for water. (Minimize Isobutane losses). PROCEED TO 2.0 START-UP AFTER TURBINE TRIP. 
APPENDIX F

DATA SHEETS FOR TESTS 


\section{GEOPRESSURED HYBRID POWER SYSTEM - MANAGEMENT REPORT DATA \\ TEST \#1}

November

\begin{tabular}{|c|c|c|c|c|c|c|c|c|c|c|c|c|}
\hline & UNITS & INST.\# & 12-Nov & 12-Nov & 12-NovI & 12-Nov & & & & & & \\
\hline & & & $7: 30$ & $7: 45$ & $8: 00$ & $8: 15$ & & & & & & \\
\hline AMBIENT TEMP & ${ }^{\circ} \mathrm{F}$ & & 68 & 68 & 69 & 71 & & & & & & \\
\hline WET BULB TEMP & ${ }^{\circ} \mathrm{F}$ & & 66 & 66 & 66 & 68 & & & & & & \\
\hline \multicolumn{13}{|l|}{ POWER } \\
\hline T-1-N GROSS & $\mathrm{KW}$ & $J 1-100$ & 464 & 463 & 467 & 459 & & & & & & \\
\hline GE-1.N GROSS & KW & & 310 & 310 & 310 & 310 & & & & & $\cdot$ & \\
\hline GE-2-NGROSS & $\mathrm{KW}$ & & 310 & 310 & 310 & 310 & & & & & & \\
\hline PARASITIC & $\overline{K W}$ & $\mathrm{J1.101}$ & 234 & 234 & 235 & 223 & & & & & & \\
\hline NET SALE TO HL\&P & $\mathrm{KW}$ & & 780 & 780 & 780 & 780 & & & & & & \\
\hline \multirow{2}{*}{ NET SALE TO HL\&P } & $\mathrm{PDG}$ & & 259 & 259 & 0259 & 0259 & & & & & & \\
\hline & $\mathrm{KW}-\mathrm{HR}$ & 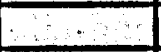 & & & & & & & & & & \\
\hline & $\square$ & & & & & & & & & & & \\
\hline \multicolumn{13}{|l|}{ BRINE } \\
\hline TO E-2.N & PSIG & $\mathrm{PI} .606$ & $5+0$ & 510 & 510 & 510 & & & & & & \\
\hline TO E-1-N & PSIG & $\mathrm{P} \mid-611$ & 510 & 510 & 510 & 510 & & & & & & \\
\hline FROM E-1-N & PSIG & $P \mid-106$ & 510 & 510 & 510 & 510 & & & & & & \\
\hline \multicolumn{13}{|l|}{ ISOBUTANE } \\
\hline IN $V-1-E$ & PSIG & PG-130 & 52 & 54 & 54 & 55 & & & & & & \\
\hline OUT OF P.1.E & PSIG & $P 1.105$ & 314 & 316 & 313 & 315 & & & & & & \\
\hline OUT OF E-1-N & PSIG & $P \mid-602$ & 290 & 290 & 290 & 290 & & & & & & \\
\hline OUT OF E-2-N & PSIG & $\mathrm{PG}-114$ & 285 & 285 & 285 & 285 & & & & & & \\
\hline OUT OF E-3-N & PSIG & PI.615 & 295 & 295 & 295 & 295 & & & & & & \\
\hline INTO T-1-E & PSIG & PI.107 & 265 & 265 & 265 & 265 & & & & & & \\
\hline OUT OF T-1-E & PSIG & $P \mid-108$ & 59 & 59 & 59 & 59 & & & & & & \\
\hline TO OA 102 & PSIG & PG-104 & 310 & 310 & 310 & 310 & & & & & & \\
\hline FROM OR 102 & PSIG & PG-105 & 150 & 150 & 150 & 150 & & & & & & \\
\hline \multicolumn{13}{|l|}{ GAS } \\
\hline TO E-3-N & PSIG & $P \mid-614$ & 0.4 & 0.4 & 0.4 & 0.4 & & & & & & \\
\hline & & & & & & + & & & & & & \\
\hline \multicolumn{13}{|l|}{ BRINE } \\
\hline TO E-2-N & ${ }^{\circ} \mathrm{F}$ & $T I .101$ & 297 & 297 & 297 & 297 & & & & & & \\
\hline TO E-1-N & ${ }^{\circ} \mathrm{F}$ & TR.113 & 207 & 207 & 207 & 206 & & & & & & \\
\hline FROM E-1-N & ${ }^{\circ} \mathrm{F}$ & TR-102 & 148 & 148 & 148 & 148 & & & & & & \\
\hline & & & & & & & & & & & & \\
\hline
\end{tabular}




\section{GEOPRESSURED HYBRID POWER SYSTEM - MANAGEMENT REPORT DATA \\ TEST \#1}

November

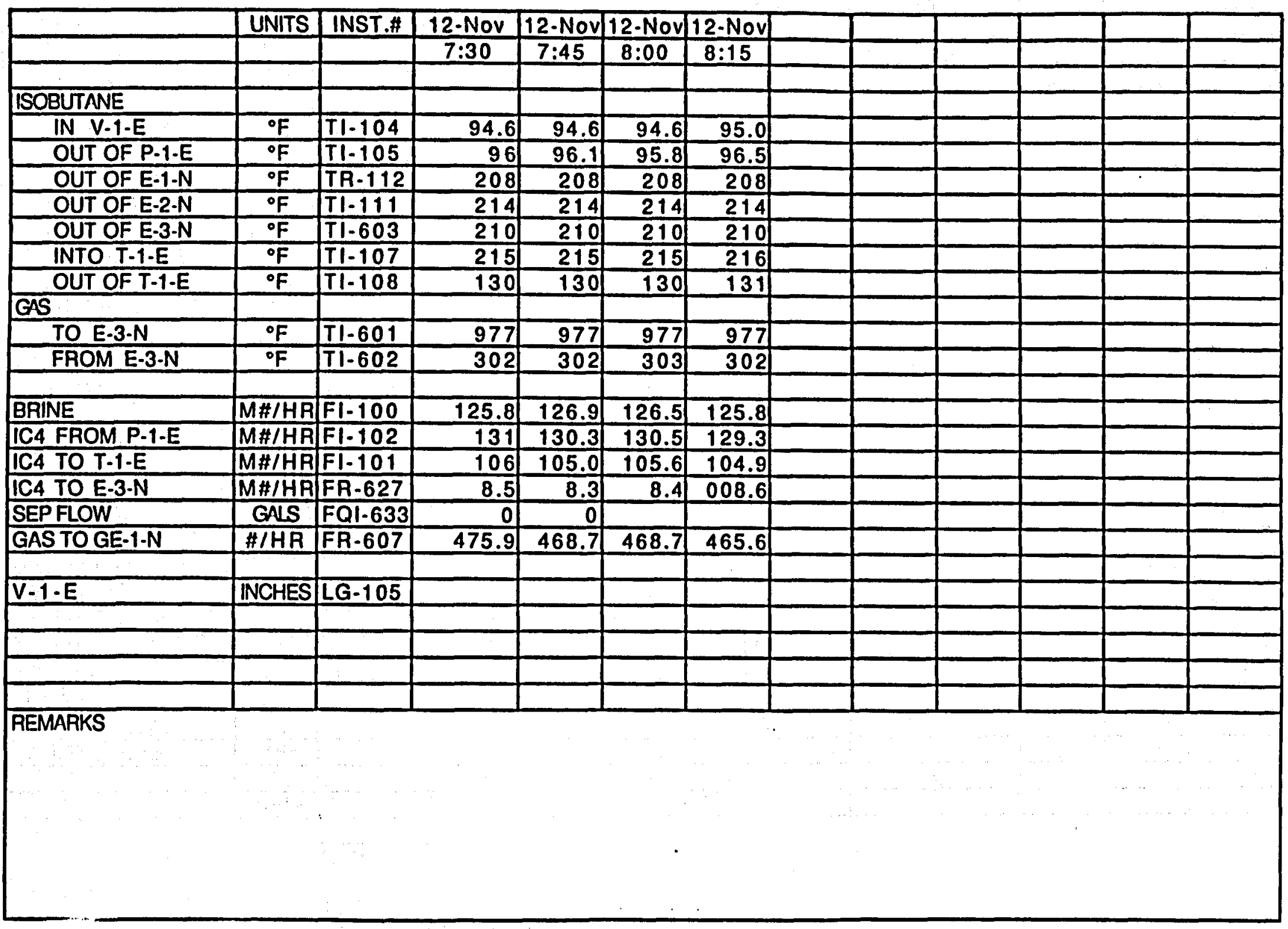


GEOPRESSURED HYBRID POWER SYSTEM - MANAGEMENT REPORT DATA TEST \#3

December 29, 1989

\begin{tabular}{|c|c|c|c|c|c|c|c|c|c|c|c|}
\hline & UNITS & INST.\# & 29-Dec & 29-Dec & 29-Dec & 29-Dec & 29-Dec & 29-Dec & 29-Dec & 29-Dec & 29-Dec \\
\hline & & & 1900 & 1915 & 1930 & 2000 & 2015 & 2030 & 2045 & 2100 & 2115 \\
\hline AMBIENT TEMP & ${ }^{\circ} \mathrm{F}$ & & 63 & 62 & 61 & 60 & 61 & 61 & 61 & 61 & \\
\hline WET BULB TEMP & ${ }^{\circ} \mathrm{F}$ & & 62 & 61 & 61 & 60 & 60 & 60 & 61 & 61 & \\
\hline \multicolumn{12}{|l|}{ POWEA } \\
\hline T.1-N GROSS & $\mathrm{KW}$ & $J 1-100$ & 502 & 501 & 504 & 501 & 499 & 496 & 494 & 494 & \\
\hline GE-1-N GROSS & $\mathrm{KW}$ & & 5 & 5 & 5 & 5 & 5 & 5 & 15 & 15 & \\
\hline GE-2-N GROSS & $\mathrm{KW}$ & & 260 & 260 & 260 & & & & 15 & 15 & \\
\hline PARASITIC & $\mathrm{KW}$ & $J 1-101$ & 242 & 242 & 252 & 243 & 242 & 242 & 252 & 242 & \\
\hline NET SALE TOHL\&P & $\mathrm{KW}$ & & 200 & 500 & 500 & 300 & 300 & 300 & 240 & 240 & \\
\hline \multirow[t]{2}{*}{ NET SALE TO HL\&P } & FDG & & 813 & 0813 & 0813 & 0813 & 0813 & 0813 & 0813 & 0813 & \\
\hline & $\mathrm{KW} \cdot \mathrm{HA}$ & & & & & & & & 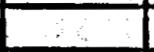 & & \\
\hline & & & & & & & & & & & \\
\hline \multicolumn{12}{|l|}{ BRINE } \\
\hline TO E-2-N & PSIG & $\mathrm{Pl} .606$ & 500 & 500 & 500 & 500 & 500 & 500 & 500 & 500 & \\
\hline TO E-1-N & PSIG & $P \mid-611$ & 490 & 490 & 490 & 490 & 490 & 490 & 490 & 490 & \\
\hline FAOM E-1.N & PSIG & $P \mid-106$ & 491 & 490 & 490 & 490 & 489 & 489 & 490 & 488 & \\
\hline \multicolumn{12}{|l|}{ ISOBUTANE } \\
\hline IN V.1.E & PSIG & PG-130 & 56 & 56 & 55 & 54 & 54 & 54 & 54 & 53 & \\
\hline OUT OF P-1.E & PSIG & PI.105 & 357 & 362 & 362 & 363 & 361 & 364 & 365 & 364 & \\
\hline OUT OF E-1-N & PSIG & PI.602 & 290 & 290 & 290 & 290 & 290 & 290 & 290 & 290 & \\
\hline OUT OF E-2-N & PSIG & PG-114 & 275 & 275 & 275 & 270 & 275 & 272 & 275 & 278 & \\
\hline OUT OF E-3-N & PSIG & $P \mid-615$ & 290 & 290 & 290 & 290 & 290 & 290 & 292 & 290 & \\
\hline INTO T-1-E & PSIG & $P \mid-107$ & 261 & 262 & 261 & 262 & 262 & 261 & 261 & 262 & \\
\hline OUT OF T-T-E & PSIG & $P /-108$ & 58 & 57 & 57 & 56 & 56 & 56 & 55 & 55 & \\
\hline TO OR 102 & PSIG & PG-104 & 62 & 61 & 61 & 60 & 60 & 60 & 60 & 61 & \\
\hline FROM OA 102 & PSIG & PG.105 & 48 & 45 & 45 & 45 & 45 & 45 & 45 & 45 & \\
\hline \multicolumn{12}{|l|}{ GAS } \\
\hline TO E-3-N & PSIG & Pl-614 & 0.6 & 0.1 & 0.1 & 0.1 & 0.1 & 0.1 & 0 & 0 & \\
\hline & & & & & & & & & & & \\
\hline \multicolumn{12}{|l|}{ BRINE } \\
\hline TO E-2-N & $\circ F$ & $\mathrm{~T} 1-101$ & 297 & 297 & 297 & 297 & 297 & 297 & 297 & 297 & \\
\hline TO E-1.N & ${ }^{\circ} \mathrm{F}$ & TR-113 & 214 & 214 & 214 & 214 & 214 & 214 & 214 & 214 & \\
\hline FROM E-1-N & ${ }^{\circ} \mathrm{F}$ & TR.102 & 150 & 150 & 150 & 150 & 151 & 151 & 152 & 151 & \\
\hline & & & & & & & & & & & \\
\hline
\end{tabular}


GEOPRESSURED HYBRID POWER SYSTEM - MANAGEMENT REPORT DATA

TEST \#3

December 29, 1989

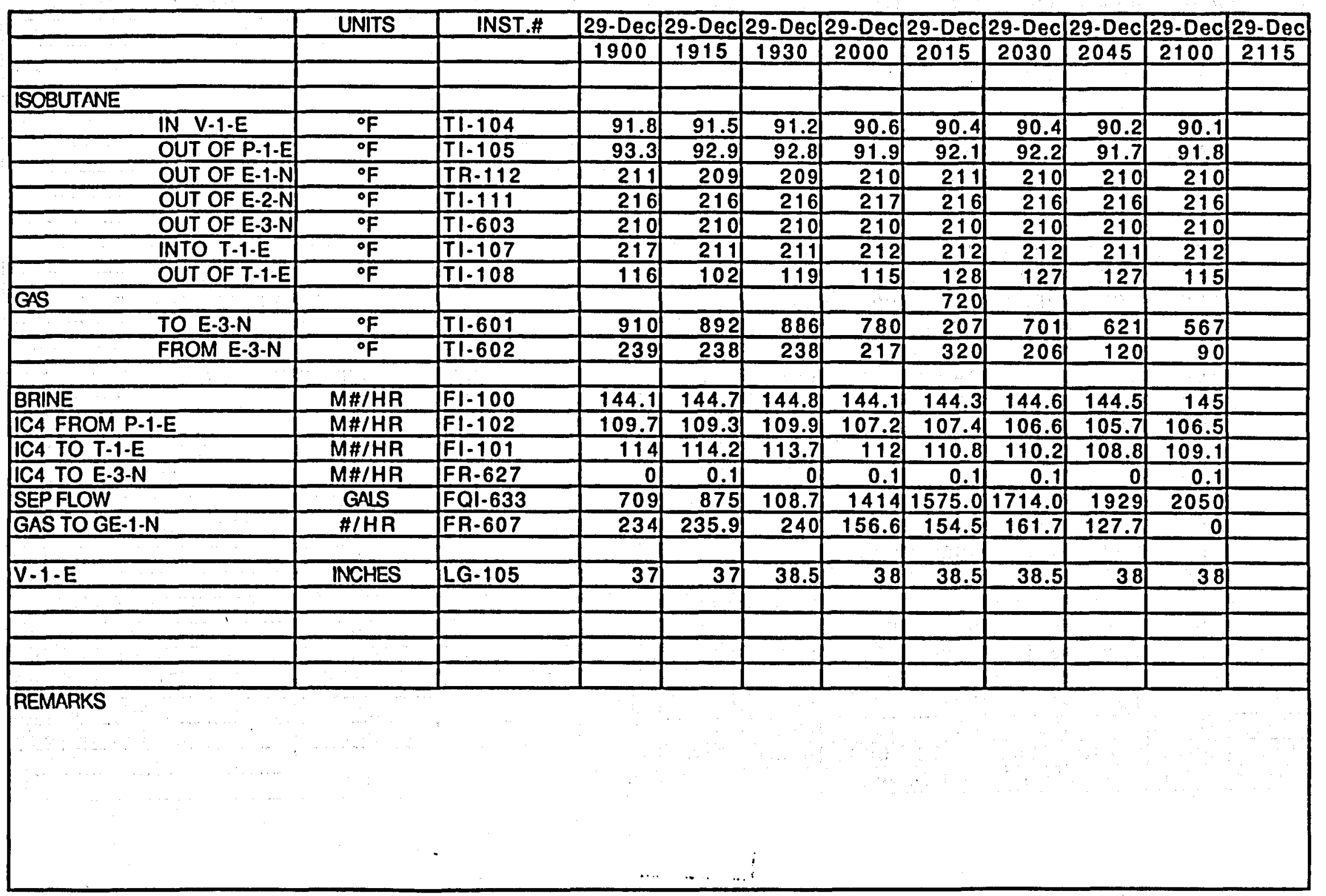


GEOPRESSURED HYBRID POWER SYSTEM - MANAGEMENT REPORT DATA

TEST \#3

January 3, 1990

\begin{tabular}{|c|c|c|c|c|c|c|c|c|c|c|c|c|}
\hline & UNITS & INST.\# & 3-Jan & $3-\operatorname{Jan}$ & $3-\mathrm{Jan}$ & $3-\mathrm{Jan}$ & & & & & & \\
\hline & & & $10: 30$ & $11: 00$ & $11: 30$ & $12: 00$ & & & & & & \\
\hline AMBIENT TEMP & of & & 64 & 64 & 64 & 64 & & & & & & \\
\hline WET BULB TEMP & ${ }^{\circ} \mathrm{F}$ & & 64 & 64 & 64 & 64 & & & & & & \\
\hline POWER & & & & & & & & & & & & \\
\hline T-1-N GROSS & $\mathrm{KW}$ & $J 1.100$ & 513 & 513 & 509 & 508 & & & & & & \\
\hline GE-1-N GROSS & $\mathrm{KW}$ & & 340 & 340 & 340 & 340 & & & & & & \\
\hline GE-2-NGROSS & $\mathrm{KW}$ & & 340 & 340 & 340 & 340 & & & & & & \\
\hline PARASITIC & $\mathrm{KW}$ & $\$ 1-101$ & 241 & 252 & 253 & 253 & & & & & & \\
\hline NET SALE TO HL\&P & $\mathrm{KW}$ & & 910 & 920 & 920 & 920 & & & & & & \\
\hline NET SALE TO HL\&P & PDG & & 0919 & 0920 & 0920 & 0921 & & & & & & \\
\hline & KW.HF & & & & & & & & & & & \\
\hline & & & & & & 4 & & & & & & \\
\hline BRINE & & & & & & & & & & & & \\
\hline TO E-2.N & PSIG & $P 1-606$ & 500 & 500 & 505 & 500 & & & & & & \\
\hline TO E-1.N & PSIG & $P \mid-611$ & 490 & 495 & 495 & 495 & & & & & & \\
\hline FROM E-1-N & PSIG & $P I-106$ & 490 & 495 & 489 & 495 & & & & & & \\
\hline ISOBUTANE & & & & & & & & & & & & \\
\hline IN V-1-E & PSIG & $P G-130$ & 56 & 56 & 56 & 56 & & & & & & \\
\hline OUT OF P.1-E & PSIG & $\mathrm{Pl} 1.105$ & 365 & 361 & 354 & 358 & & & & & & \\
\hline OUT OF E-1.N & PSIG & $P \mid-602$ & 295 & 295 & 295 & 295 & & & & & & \\
\hline OUT OF E-2-N & PSIG & $P G-114$ & 280 & 280 & 280 & 280 & & & & & & \\
\hline OUT OF E-3.N & PSIG & $\mathrm{P} 1.615$ & 295 & 295 & 295 & 295 & & & & & & \\
\hline INTO T-1-E & PSIG & $P 1.107$ & 265 & 264 & 265 & 265 & & & & & & \\
\hline OUT OF T-1-E & PSIG & $\mathrm{PI} / 108$ & 59 & 59 & 59 & 59 & & & & & & \\
\hline TO OR 102 & PSIG & PG-104 & 65 & 65 & 65 & 65 & & & & & & \\
\hline FROM OR 102 & PSIG & PG.105 & 45 & 45 & 45 & 45 & & & & & & \\
\hline GAS & & & & & & & & & & & & \\
\hline TO E-3-N & PSIG & $P 1.614$ & 0.7 & 0.7 & 0.7 & 0.7 & & & & & & \\
\hline & & & & & & & & & & & & \\
\hline BRINE & & & & & & & & & & & & \\
\hline TO E-2-N & ${ }^{\circ} \mathrm{F}$ & $T 1.101$ & 296 & 296 & 296 & 296 & & & & & & \\
\hline TO E-1.N & ${ }^{\circ} \mathrm{F}$ & TR-113 & 216 & 217 & 215 & 215 & & & & & & \\
\hline FROM E-1.N & ${ }^{\circ} \mathrm{F}$ & TR.102 & 151 & 151 & 151 & 152 & & & & & & \\
\hline & & & & & & & & & & & & \\
\hline
\end{tabular}


GEOPRESSURED HYBRID POWER SYSTEM - MANAGEMENT REPORT DATA TEST \#3

January 3,1990

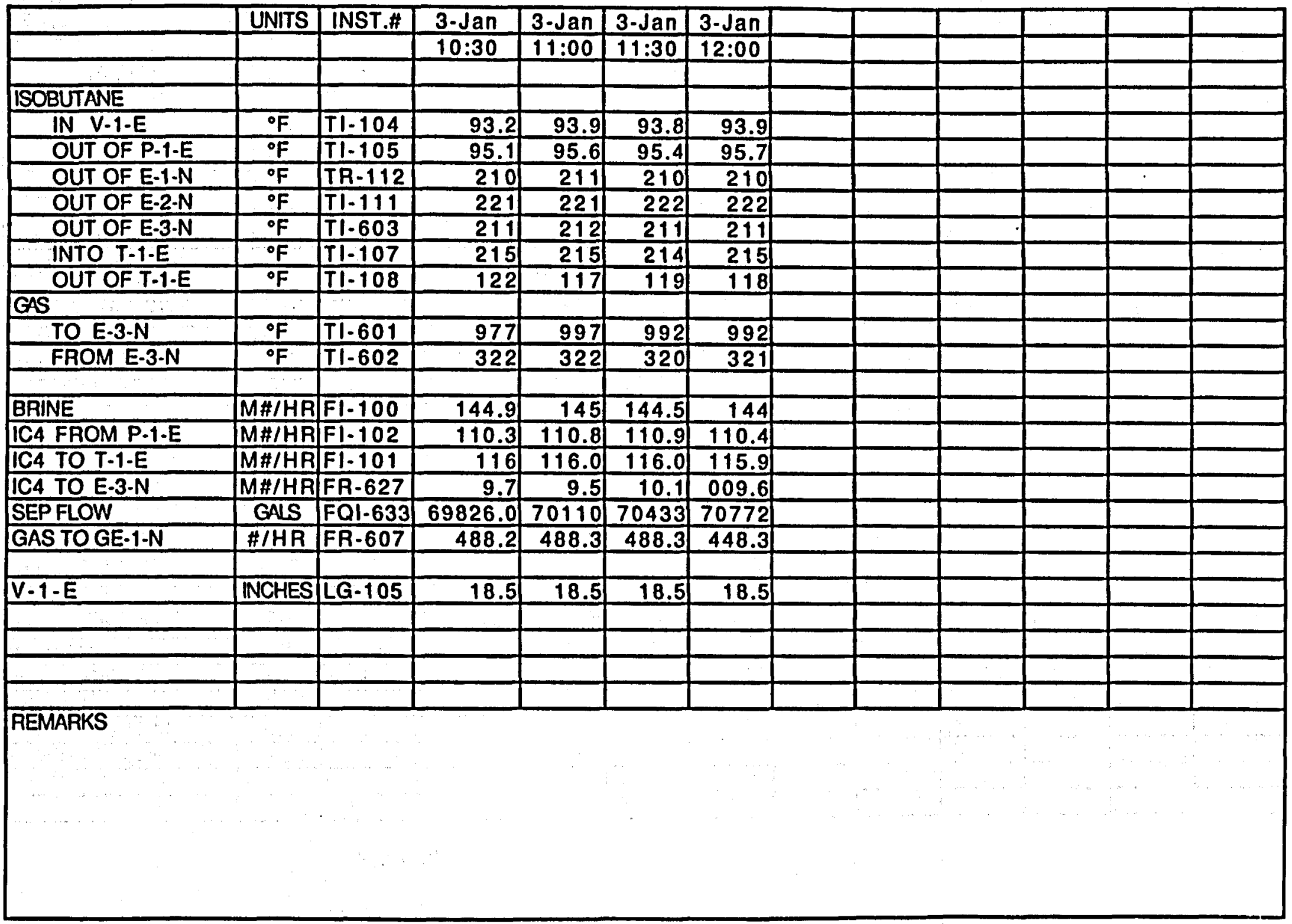


LOW EXHAUST GAS TEST

GEOPRESSURED HYBRID POWER SYSTEM - MANAGEMENT REPORT DATA

TEST 4

May 9, 1990

\begin{tabular}{|c|c|c|c|c|c|c|c|c|c|c|c|c|c|}
\hline & UNITS & INST.\# & & $05: 00$ & $07: 00$ & $08: 00$ & & 09:00 & 09:30 & $10: 00$ & & & \\
\hline & & & $\because$ & $100 \%$ & $100 \%$ & $100 \%$ & & $75 \%$ & $75 \%$ & $75 \%$ & & & \\
\hline & & & & & & & & & & & & & \\
\hline AMBIENT TEMP & ${ }^{\circ} \mathrm{F}$ & & & 64 & 65 & 72 & & 78 & 82 & 80 & & & \\
\hline WET BULB TEMP & ${ }^{\circ} \mathrm{F}$ & $\ldots$ & . & 63 & 63 & 68 & & 73 & 72 & 73 & & & \\
\hline \multicolumn{14}{|l|}{ POWEA } \\
\hline T-1-N GROSS & $\mathrm{KW}$ & $\$ 1.100$ & $\ldots$ & 529 & 528 & 500 & & 500 & 495 & 500 & & & \\
\hline GE-1-N GROSS & $\mathrm{KW}$ & \begin{tabular}{|l}
$\ldots \ldots \ldots$ \\
\end{tabular} & $\cdots$ & 310 & 310 & 300 & & 100 & 100 & 100 & & & \\
\hline GE-2-N GROSS & $\mathrm{KW}$ & & $\cdots$ & 340 & 340 & 340 & & 340 & 340 & 340 & & & \\
\hline PARASITIC & $\mathrm{KW}$ & J1-101 & & 242 & 252 & 238 & & 250 & 248 & 249 & & & \\
\hline NET SALE TOHL\&P & $\mathrm{KW}$ & & $\ldots \ldots$ & 900 & 900 & 870 & & 650 & 650 & 645 & & & \\
\hline NET SALE TO HL\&P & FDG & -1 & 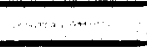 & 3169 & 3171 & 3172 & & 3172 & 3173 & 3163 & & & \\
\hline 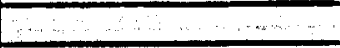 & KW-HR & & & $-\ldots$ & 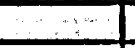 & $\quad 1$ & & $\cdots$ & $\therefore \cdots$ & +1 & & & \\
\hline$\overline{2}$ & & & $\cdots$ & & & & & $\because$ & & c & & & \\
\hline \multicolumn{14}{|l|}{ BAINE } \\
\hline TO E-2-N & PSIG & P/.606 & & 505 & 505 & 505 & & 505 & 505 & 509 & & & \\
\hline TO E-1-N & PSIG & $\mathrm{Pl}-611$ & & 495 & 495 & 500 & & 495 & 495 & 492 & & & \\
\hline FROM E-1-N & PSIG & $P \mid-106$ & $-n$ & 482 & 479 & 480 & & 490 & 490 & 475 & & & \\
\hline \multicolumn{14}{|l|}{ ISOBUTANE } \\
\hline IN $V-1-E$ & PSIG & PG-130 & & 60 & 60 & 62 & & 62 & 62 & 63 & & & \\
\hline OUT OF P-1-E & PSIG & P/.105 & 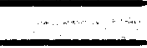 & 340 & 340 & 347 & & 356 & 358 & 355 & & & \\
\hline OUT OF E-1-N & PSIG & $P \mid-602$ & $\ldots$ & 310 & 310 & 310 & & 309 & 310 & 309 & & & \\
\hline OUT OF E-2-N & PSIG & PG.114 & 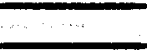 & 280 & 280 & 280 & & 280 & 280 & 280 & & & \\
\hline OUT OF E-3-N & PSIG & P/.615 & $2 \ldots$ & 290 & 290 & 290 & & 285 & 285 & 288 & & & \\
\hline INTO T-1.E & PSIG & PI-107 & & 268 & 267 & 266 & & 261 & 262 & 262 & & & \\
\hline OUT OF T-1.E & PSIG & $P \mid-108$ & & 54 & 54 & 57 & & 55 & 57 & 57 & & & \\
\hline TO OA 102 & PSIG & PG-104 & . & 60 & 60 & 60 & & 60 & 60 & 61 & & & \\
\hline FROM OA 102 & PSIG & PG-105 & & 60 & 60 & 60 & & 60 & 60 & 61 & & & \\
\hline \multicolumn{14}{|l|}{ GAS } \\
\hline TO E-3-N & PSIG & PI.614 & & .2 & .2 & .2 & & .2 & .2 & .2 & & & \\
\hline 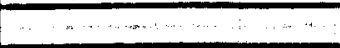 & & & & & & & & & & & & & \\
\hline \multicolumn{14}{|l|}{ BRINE } \\
\hline TO E-2.N & of & $\mathrm{Tl}-101$ & & 298 & 298 & 298 & & 297 & 297 & 297 & & & \\
\hline TO E.1-N & ${ }^{\circ} \mathrm{F}$ & TR-113 & & 218 & 218 & 218 & & 216 & 216 & 217 & & & \\
\hline FROM E-1.N & ${ }^{\circ} \mathrm{F}$ & TR-102 & & 150 & 149 & 151 & & 151 & 152 & 152 & & & \\
\hline & & & & & & & & & & & & & \\
\hline & & & & & & & & & & & & & \\
\hline
\end{tabular}

$F \cdot 7$ 
LOW EXHAUST GAS TEST

GEOPRESSURED HYBRID POWER SYSTEM - MANAGEMENT REPORT DATA

TEST 4

May 9, 1990

\begin{tabular}{|c|c|c|c|c|c|c|c|c|c|c|c|c|c|}
\hline & UNITS & INST.\# & & $05: 00$ & $07: 00$ & $08: 00$ & & $09: 00$ & $09: 30$ & $10: 00$ & & & \\
\hline & & & & $100 \%$ & $100 \%$ & $100 \%$ & & $75 \%$ & $75 \%$ & $75 \%$ & & & \\
\hline & & & & & & & & & & & & & \\
\hline & & & & & & & & & & & & & \\
\hline & & & & & & & & & & & & & \\
\hline \multicolumn{14}{|l|}{ ISOBUTANE } \\
\hline IN V.1.E & ${ }^{\circ} \mathrm{F}$ & $T 1.104$ & & 95.6 & 93.7 & 97.2 & & 96.3 & 96.5 & 96.9 & & & \\
\hline OUT OF P-1-E & ${ }^{\circ} \mathrm{F}$ & $T 1.105$ & & 96.1 & 965 & 101.3 & & 97.7 & 99.5 & 100.1 & & & \\
\hline OUT OF E-1-N & ${ }^{\circ} \mathrm{F}$ & TR-112 & & 212 & 213 & 212 & & 212 & 211 & 211 & & & \\
\hline OUT OF E-2-N & ${ }^{\circ} \mathrm{F}$ & $T 1.111$ & & 219 & 218 & 219 & & 219 & 219 & 220 & & & \\
\hline OUT OF E.3-N & ० $\mathrm{F}$ & $T 1.603$ & & 212 & 212 & 212 & & 212 & 211 & 211 & & & \\
\hline INTO T-1-E & of & $T 1.107$ & & 215 & 215 & 215 & & 215 & 214 & 214 & & & \\
\hline OUT OF T-1-E & of & $T 1-108$ & & 135 & 136 & 138 & & 138 & 138 & 138 & & & \\
\hline \multicolumn{14}{|l|}{ GAS } \\
\hline TO E.3.N & ० $\mathrm{F}$ & $T 1.601$ & & 1007 & 1010 & 1007 & & 968 & 962 & 955 & & & \\
\hline FAOM E-3-N & of & $T 1.602$ & & 363 & 363 & 361 & & 325 & 325 & 324 & & & \\
\hline & & & & & & & & & & & & & \\
\hline BRINE & $\overline{M \# / H R}$ & $F 1.100$ & & 148.7 & 151.2 & 150.2 & & 149.7 & 150.3 & 147.5 & & & \\
\hline IC4 FROM P-1.E & M\#/HR & $F \mid-102$ & & 119.5 & 120.1 & 117.9 & & 118.2 & 114.4 & 120 & & & \\
\hline IC4 TO T-1.E & $M \# / H A$ & $F \mid .101$ & & 122.7 & 122.9 & 122.5 & & 122.0 & 121.8 & 122.2 & & & \\
\hline IC4 TO E-3.N & M\#/HA & FR-627 & & 11.0 & 11.1 & 9.5 & & 6.6 & 6.9 & 6.6 & & & \\
\hline SEPFLOW & GAS & FQI-633 & & 782309 & 788926 & 793675 & & 7970923 & 7986162 & 8004590 & & & \\
\hline \multirow[t]{2}{*}{ GAS TO GE-1-N } & $\# / \mathrm{HR}$ & FR.607 & & 492.4 & 490.3 & \begin{tabular}{|l|}
486.2 \\
\end{tabular} & & 364.6 & $\begin{array}{r}363.6 \\
\end{array}$ & \begin{tabular}{|l|}
366.7 \\
\end{tabular} & & & \\
\hline & & & & & & & & & & & & & \\
\hline $\mathrm{V} \cdot 1 \cdot \mathrm{E}$ & INCHES & LG.105 & & 29.5 & 32 & 29.5 & & 22 & 22 & 22.5 & & & \\
\hline & & & & & & & & & & & & & \\
\hline & & & & & & & & & & & & & \\
\hline & & & & & & & & & & & & & \\
\hline & & & & & & & & & & & & & \\
\hline
\end{tabular}

AFMARKS

F -8 
LOW EXHAUST GAS TEST

GEOPRESSURED HYBRID POWER SYSTEM - MANAGEMENT REPORT DATA

TEST 4

May 9,1990

\begin{tabular}{|c|c|c|c|c|c|c|c|c|c|c|c|}
\hline & INST.\# & & $11: 00$ & $11: 30$ & 12:00 & $01: 00$ & $01: 30$ & $02: 00$ & $15: 00$ & $15: 30$ & $16: 00$ \\
\hline & & & $50 \%$ & $50 \%$ & $50 \%$ & $25 \%$ & $25 \%$ & $25 \%$ & $0 \%$ & $0 \%$ & $0 \%$ \\
\hline & & & & & & & & & & & \\
\hline AMBIENT TEMP & & & 82 & 82 & 85 & 86 & 85 & 86 & 86 & 88 & 89 \\
\hline WET BULB TEMP & & & 73 & 74 & 76 & 76 & 76 & 76 & 76 & 78 & 77 \\
\hline POWEA & & & & & & & & & $\ldots$ & & \\
\hline T-1.N GROSS & $\mathrm{J1.100}$ & & 490 & 489 & 482 & 472 & 465 & 471 & 462 & 462 & 462 \\
\hline GE-1-NGROSS & & 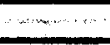 & 0 & 0 & 0 & 0 & 0 & 0 & 0 & 0 & $\cdots 0$ \\
\hline GE-2-N GROSS & & & 340 & 340 & 340 & 95 & 90 & 90 & 0 & 0 & 0 \\
\hline PARASITIC & J1-101 & & 248 & 238 & 247 & 246 & 246 & 247 & 245 & 236 & 247 \\
\hline NET SALE TO HLEP & & & 560 & 560 & 570 & 320 & 310 & 320 & 220 & 220 & 220 \\
\hline NET SALE TO HL\&P. & & & 3174 & 3174 & 3174 & 3175 & 3175 & 3175 & 3175 & 3176 & 3176 \\
\hline $\begin{array}{ccc}+5 \\
\end{array}$ & & & $\ldots$ & $\therefore$ & -2 & $m_{-1}$ & $\cdots \cdots$ & $\cdots$ & 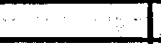 & 2 & \\
\hline & & & - & & & & & & & & \\
\hline BAINE & & & $=$ & $\cdots$ & .... & & $\cdots$ & $\therefore$ & & & \\
\hline TO E-2-N & $\mathrm{P} \mid-606$ & & -505 & 505 & 505 & 505 & 502 & 505 & 510 & 510 & 5.10 \\
\hline TO E-1-N & $\mathrm{P} 1.611$ & & 495 & 492 & 492 & 495 & 490 & 495 & 495 & 492 & 492 \\
\hline FROM E-1-N & $\mathrm{PI}-106$ & & 490 & 490 & 476 & 490 & 485 & 490 & 490 & 490 & 490 \\
\hline ISOBUTANE & & & & & & & 3 & & & & \\
\hline IN $V-1 . E$ & PG.130 & & 62 & 63 & 63 & 64 & 64 & 64 & 64 & 64 & 65 \\
\hline OUT OF P-1-E & $\mathrm{Pl} 1.105$ & & 366 & 361 & 361 & 370 & 362 & 372 & 367 & 373 & 375 \\
\hline OUT OF E-1.N & $\mathrm{PI} \cdot 602$ & & 310 & 309 & 310 & 305 & 310 & 310 & 310 & 310 & 310 \\
\hline OUT OF E-2-N & $P G-114$ & & 282 & 280 & 280 & 280 & 280 & 280 & 280 & 280 & 280 \\
\hline OUT OF E-3-N & $P \mid-615$ & & 290 & 285 & 285 & 283 & 285 & 265 & 260 & 240 & 225 \\
\hline INTO T.1.E & $P 1-107$ & & 265 & 266 & 264 & 264 & 264 & 265 & 266 & 267 & 267 \\
\hline OUT OF T-1-E & $P 1.108$ & & 58 & 59 & 59 & 60 & 62 & 62 & 62 & 59 & 59 \\
\hline TO OA 102 & PG.104 & & 61 & 61 & 61 & 61 & 61 & 65 & 65 & 65 & 65 \\
\hline FROM OA 102 & PG-105 & & 61 & 61 & 61 & 16 & 61 & 65 & 65 & 65 & 65 \\
\hline GAS & & & & & & & & & & & \\
\hline TO E-3-N & PI-614 & & .0 & .0 & .0 & .0 & .0 & .0 & .0 & .0 & .0 \\
\hline & & & & & & & & & & & \\
\hline BRINE & & & & & & & & & & & \\
\hline TO E-2-N & $T 1-101$ & & 297 & 297 & 297 & 297 & 297 & 297 & 297 & 297 & 297 \\
\hline TO E-1-N & TR-113 & & 217 & 216 & 217 & 216 & 216 & 216 & 216 & 216 & 216 \\
\hline FROM E-1-N & TR-102 & & 153 & 154 & 153 & 154 & 154 & 156 & 156 & 156 & 155 \\
\hline & & & & & & & & & & & \\
\hline & & & & & & & & & & & \\
\hline
\end{tabular}

$F=9$ 
LOW EXHAUST GAS TEST

GEOPRESSURED HYBRID POWER SYSTEM - MANAGEMENT REPORT DATA

TEST 4

May 9,1990

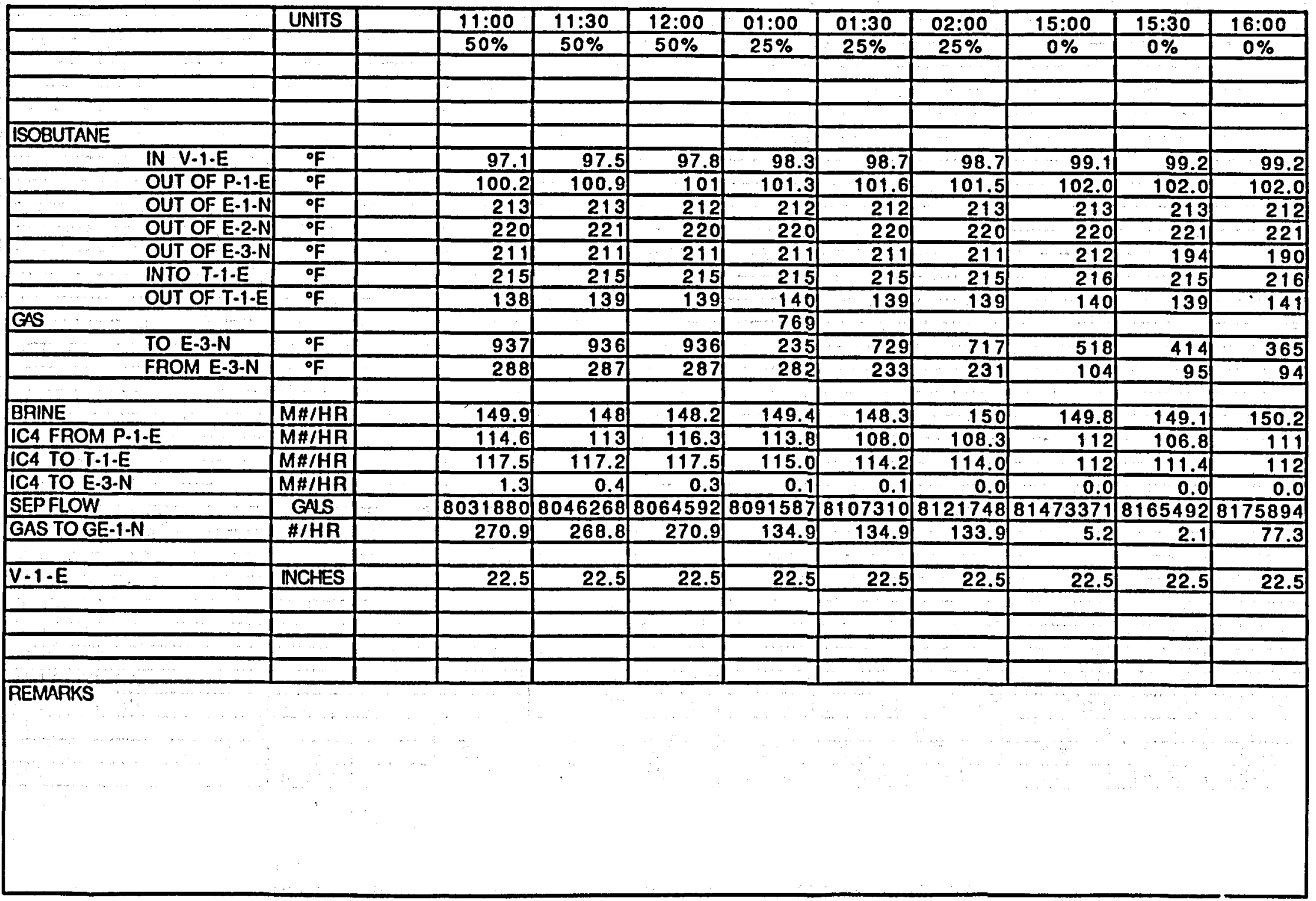


GEOPRESSUAED HYBRID POWEA SYSTEM - MANAGEMENT REPORT DATA

TEST 5

DATEBEGINNING

NOVEMBER.

58

TEST $5 C$

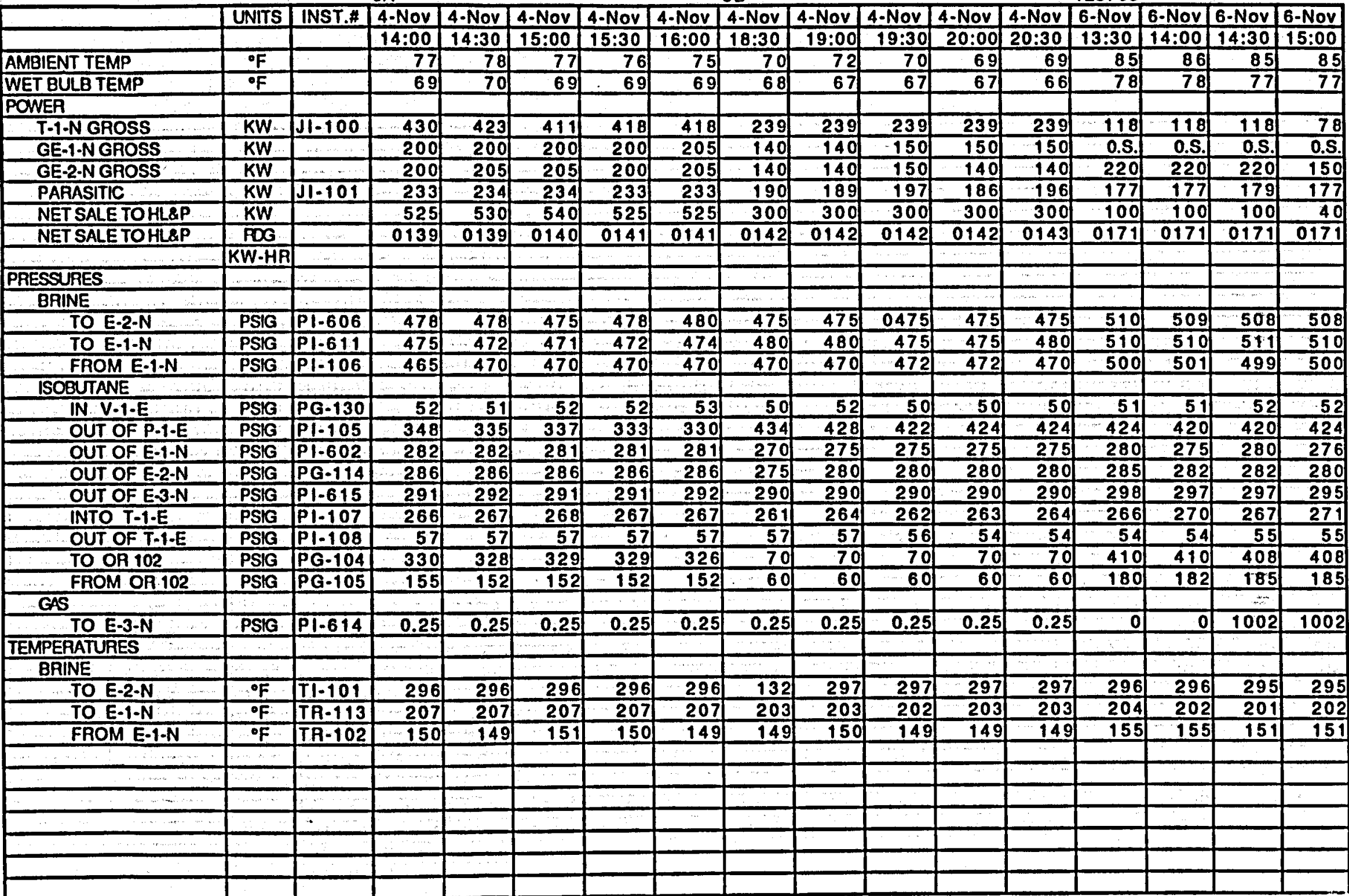

$F \cdot 11$ 


\section{GEOPRESSURED HYBRID POWER SYSTEM - MANAGEMENT REPORT DATA}

\section{TEST 5}

DATE BEGINNING

NOVEMBER

$5 \mathrm{~A}$

58

TEST 5C

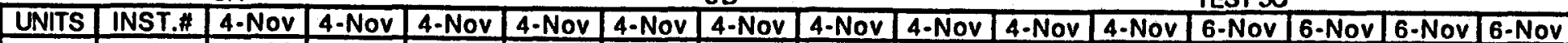

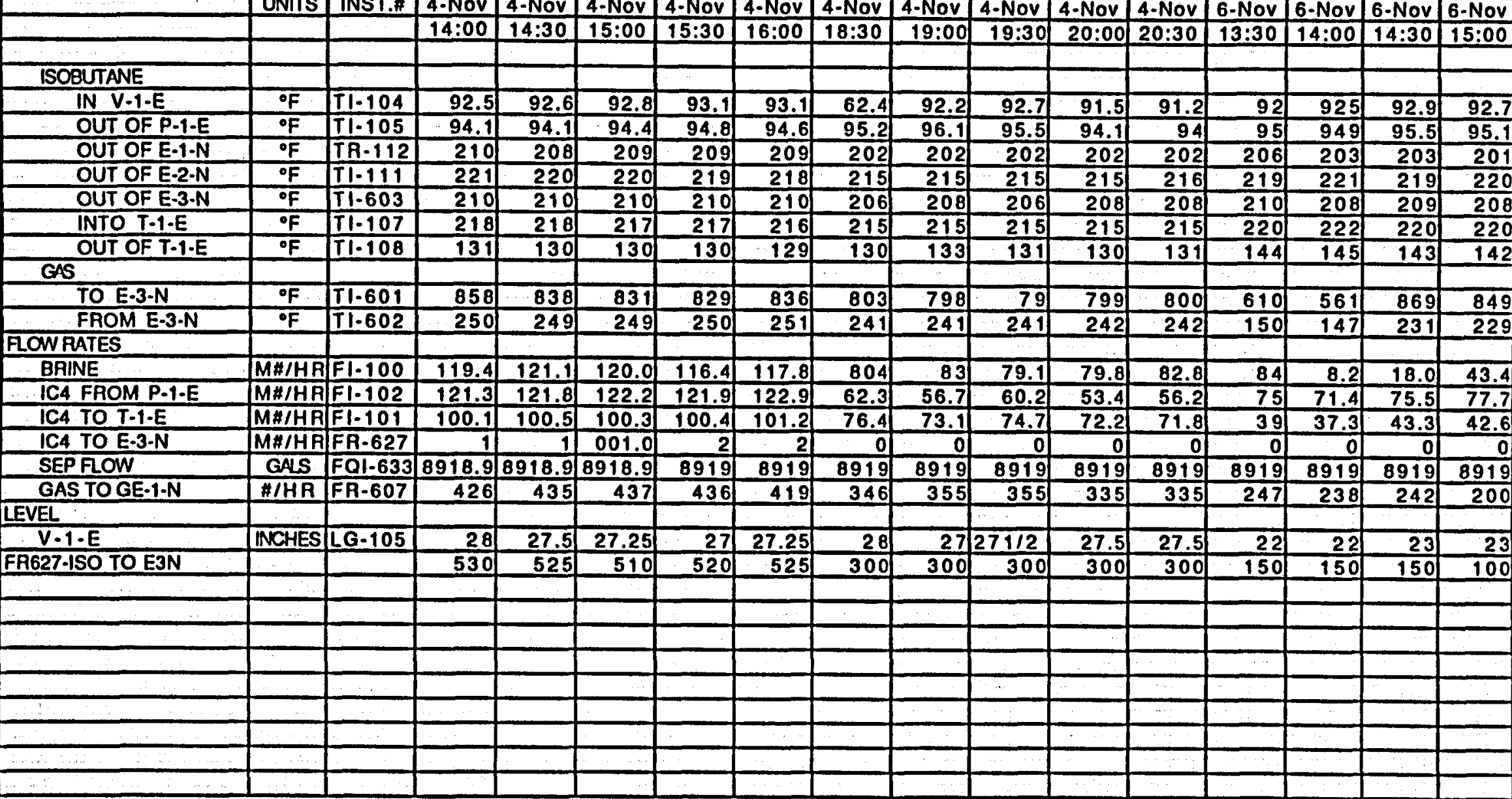

REMARKS 
GEOPRESSURED HYBRID POWER SYSTEM - MANAGEMENT REPORT DATA

TEST 6

January 4, 1990

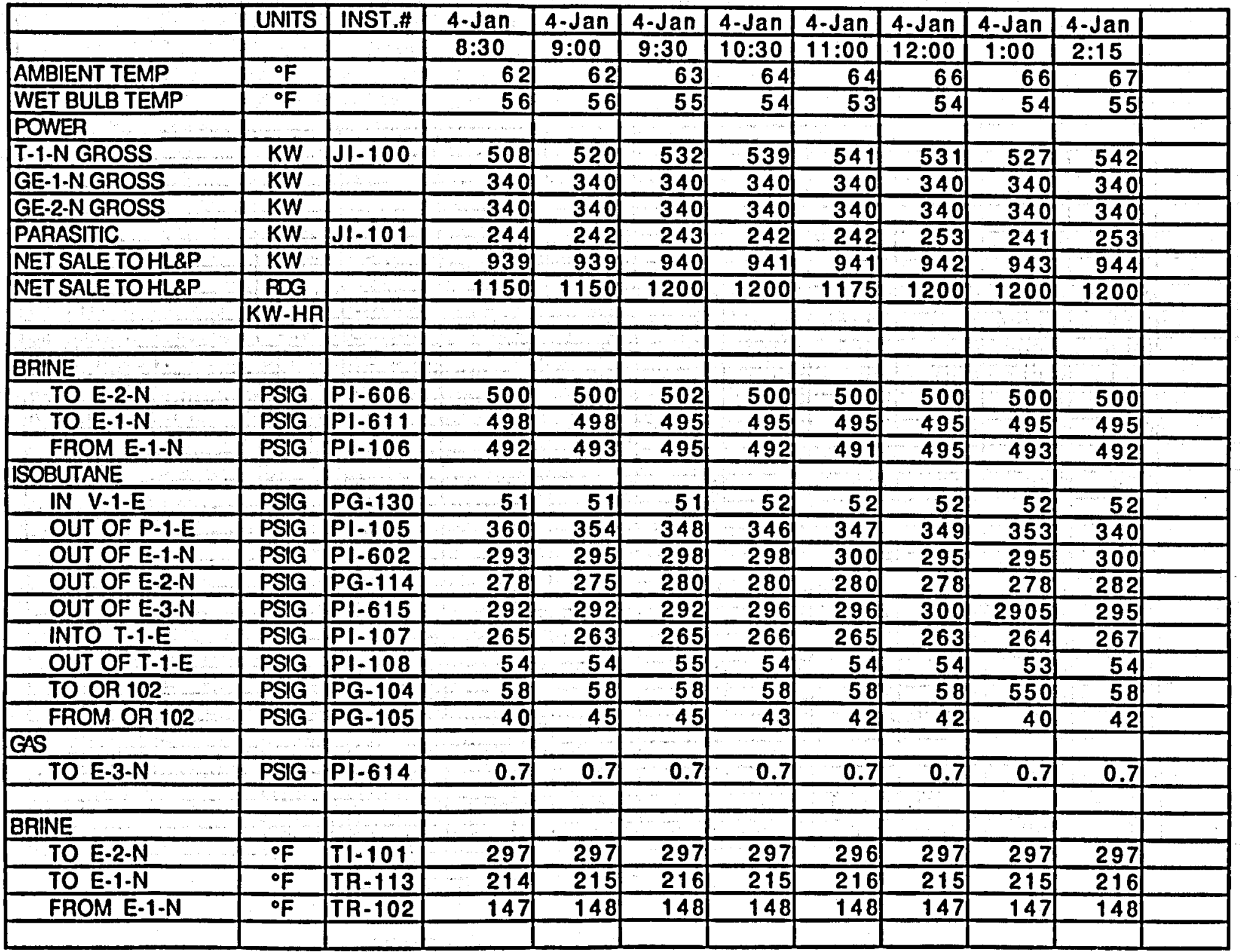

$F .13$ 
GEOPRESSURED HYBRID POWER SYSTEM - MANAGEMENT REPORT DATA

TEST 6

January 4; 1990

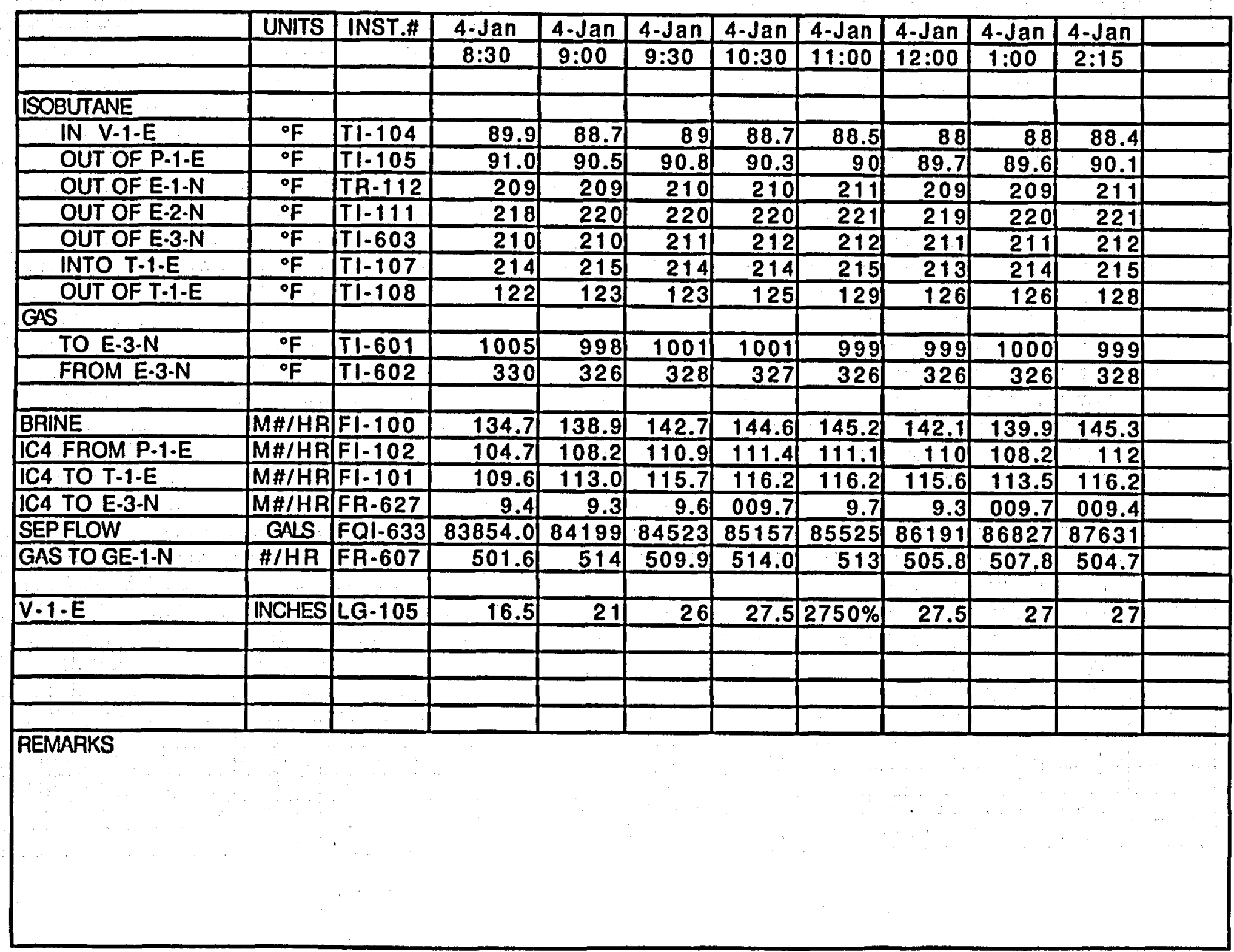

$F \cdot 14$ 


\section{GEOPRESSURED HYBRID POWER SYSTEM - MANAGEMENT REPORT DATA}

\begin{tabular}{|c|c|c|c|c|c|c|c|c|c|c|c|c|c|c|}
\hline & UNTS & INST.\# & 1000 & 1030 & 1100 & 1130 & 1200 & 1700 & 1900 & 2100 & 2300 & 0100 & 0300 & 0500 \\
\hline & & & & & & & & & & & & & & \\
\hline AMBIENT TEMP & of & & 72 & 75 & 75 & 76 & 77 & & & & & & & \\
\hline WET BULB TEMP & of & & 69 & 70 & 70 & 70 & 70 & & & & & & & \\
\hline \multicolumn{15}{|l|}{ POWER } \\
\hline T-1.N GROSS & $\mathrm{KW}$ & $J 1.100$ & 0 & 0 & 0 & 0 & 0 & & & & & & & \\
\hline GE-1-NGROSS & $\mathrm{KW}$ & & 320 & 325 & 325 & 325 & 325 & & & & & & & \\
\hline GE-2-NGROSS & $\mathrm{KW}$ & & 325 & 325 & 320 & 320 & 315 & & & & & & & \\
\hline PARASITIC & $\overline{\mathrm{KW}}$ & J1.101 & 179 & 180 & 182 & 182 & 172 & & & & & & & \\
\hline NET SALE TOHL\&P & $\mathrm{KW}$ & 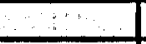 & 390 & 395 & 390 & 395 & 390 & & & & & & & \\
\hline NET SALE TOHL\&P & KW-HR & & 8 & 9 & 9 & 9 & 9 & & & & & & & \pm \\
\hline \multicolumn{15}{|l|}{ PRESSURES } \\
\hline \multicolumn{15}{|l|}{ BRINE } \\
\hline TOE-2-N & PSIG & P1.606 & 522 & 523 & 523 & 522 & 520 & & & & & & & \\
\hline TO E-1-N & PSIG & $P 1.611$ & 524 & 525 & 525 & 523 & 523 & & & & & & & \\
\hline FROM E-1-N & PSIG & $P \mid-106$ & 519 & 520 & 519 & 519 & 518 & & & & & & & \\
\hline \multicolumn{15}{|l|}{ ISOBUTANE } \\
\hline IN $V-1 \cdot E$ & PSIG & PG-130 & 62 & 64 & 62 & 63 & 63 & & & & & & & \\
\hline OUT OF P-1-E & PSIG & $\mathrm{Pl}-105$ & 357 & 357 & 377 & 360 & 358 & & & & & & & \\
\hline OUT OF E-1-N & PSIG & $P 1-602$ & 300 & 295 & 300 & 298 & 300 & & & & & & & \\
\hline OUT OF E-2-N & PSIG & PG-114 & 280 & 280 & 277 & 281 & 280 & & & & & & & \\
\hline OUT OF E-3-N & PSIG & PI-615 & 290 & 287 & 284 & 286 & 285 & & & & & & & 2 \\
\hline INTO T-1-E & PSIG & $\mathrm{P} 1.107$ & 29 & 29 & 33 & 35 & 36 & & & & & & & \\
\hline OUT OF T-1-E & PSIG & $P 1.108$ & 30 & 30 & 34 & 36 & 37 & & & & & & & \\
\hline TO OR 102 & PSIG & PG.104 & 345 & 345 & 358 & 348 & 349 & & & & & & & \\
\hline FROM OR 102 & PSIG & PG.105 & 160 & 160 & 165 & 163 & 162 & & & & & & & \\
\hline \multicolumn{15}{|l|}{ GAS } \\
\hline TO E-3.N & PSIG & $P 1.614$ & 0 & 0 & 0 & 0 & 0 & & & & & & & \\
\hline \multicolumn{15}{|l|}{ TEMPERATURES } \\
\hline \multicolumn{15}{|l|}{ BAINE } \\
\hline TO E-2-N & of & $T 1.101$ & 295 & 295 & 295 & 295 & 295 & & & & & & & \\
\hline TO E-1-N & ${ }^{\circ} \mathrm{F}$ & TR-113 & 206 & 206 & 205 & 204 & 204 & & & & & & & \\
\hline FROM E-1-N & ${ }^{\circ} \mathrm{F}$ & TA-102 & 145 & 147 & 149 & 148 & 149 & & & & & & & \\
\hline & & & & & & & & & & & & & & \\
\hline & & & & & & & & & & & & & & \\
\hline
\end{tabular}




\section{APPENDIX G}

\section{PLANT OUTAGES}

THE BEN HOLT CO. 


\section{PLANT OUTAGES}

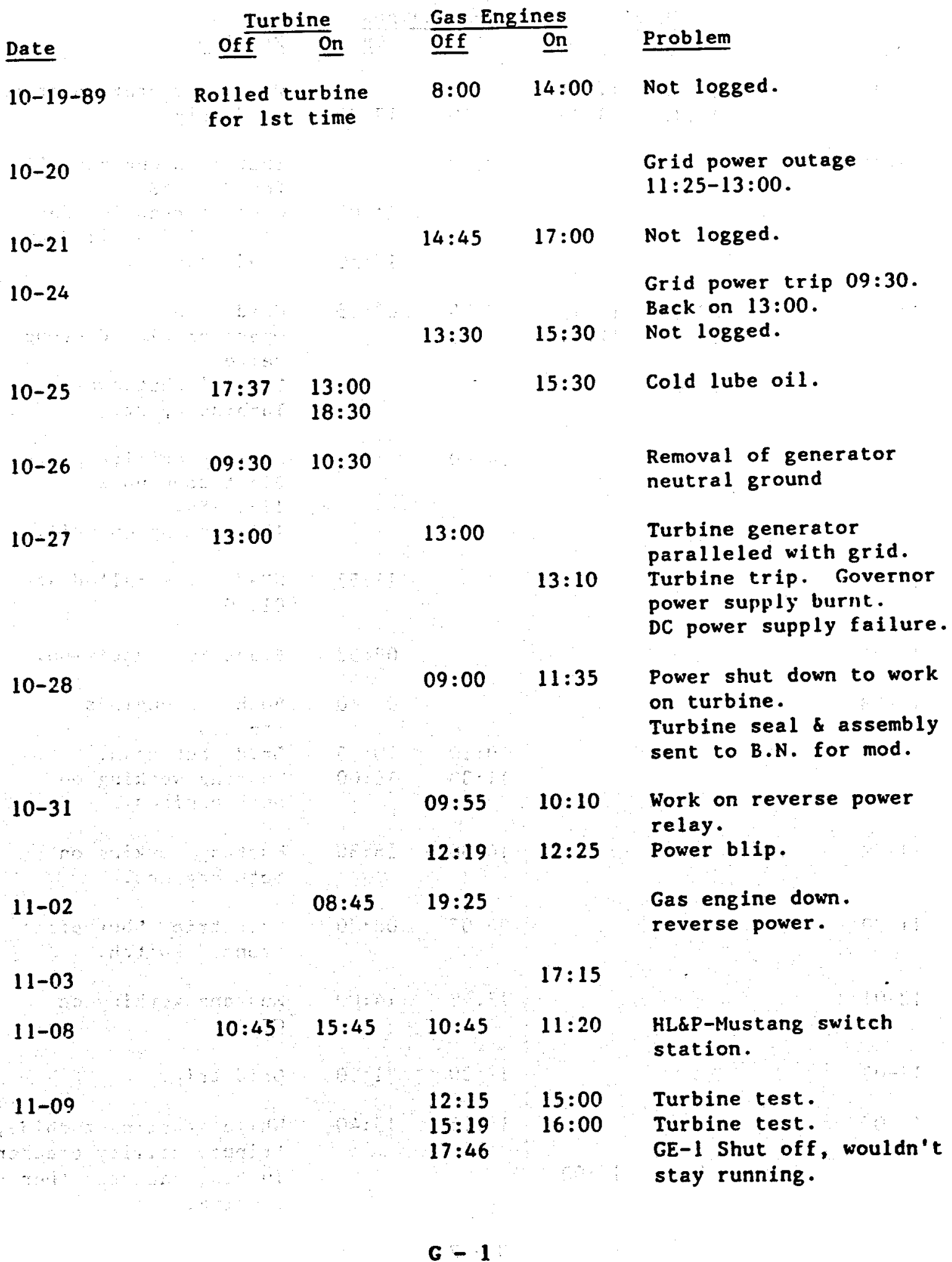


PLANT OUTAGES

\section{Date}

$11-10$

$11-12-89$

$11-15$

$11-24$

$11-25$

$11-28$

$11-29$

$11-30$

12-01.

12-03

12-07
Turbine

Off. On

$11: 50 \quad 14: 17$

$16: 10$

19:00

$16: 10$

$09: 00$

$08: 35$

$14: 10$

$12: 45$

$14: 30$

$16: 00$

$14: 00$

$08: 35$

4:

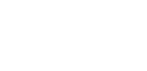

\section{Gas Engines}

Off On

$17: 00$

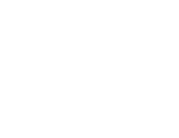




\section{PLANT OUTAGES}

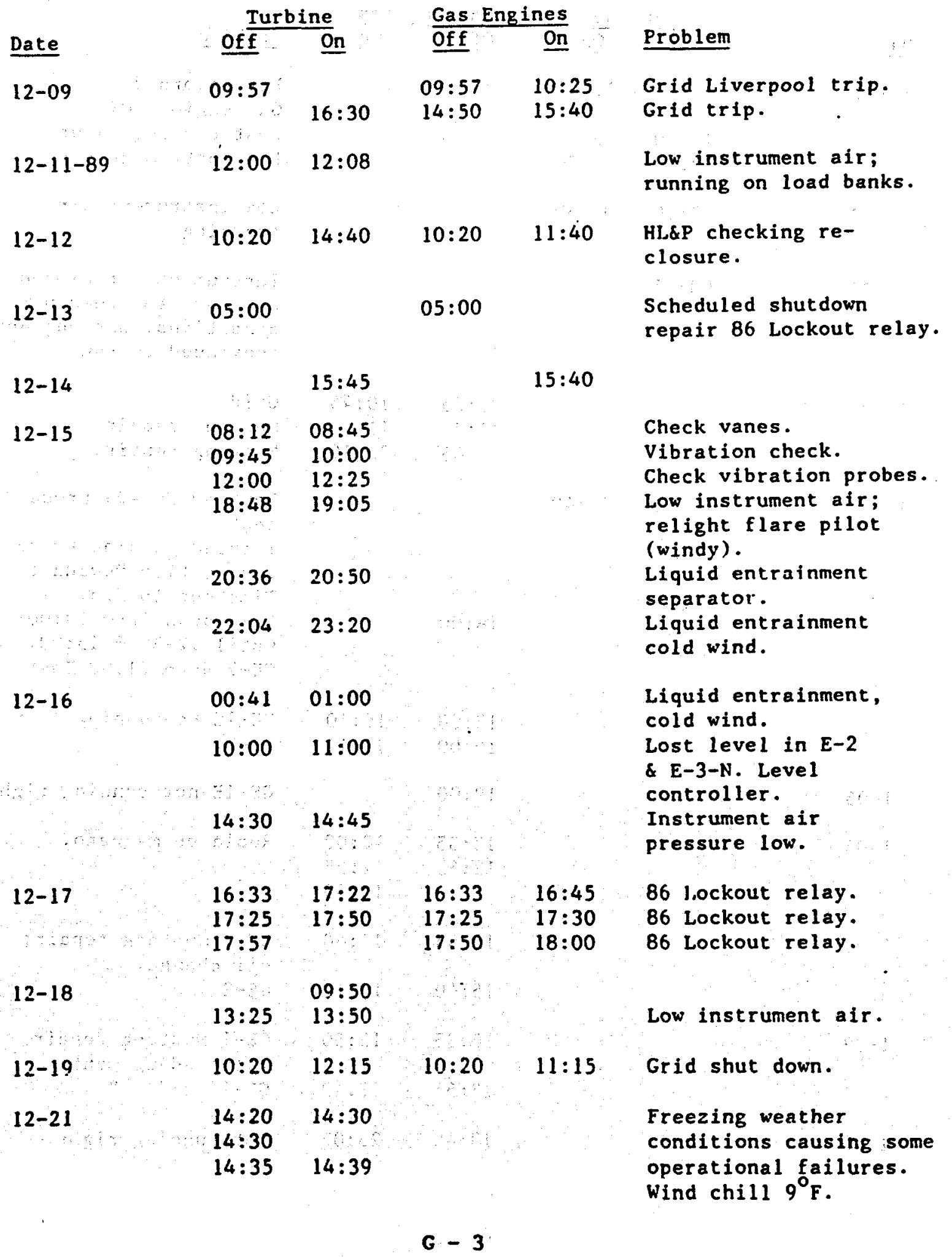




\section{PLANT OUTAGES}

Date

12-22

$12-26$

$12-29$

$1-03$

$1-05$

$1-06$

$1-08$

$1-09$

$1-10$
Gas Engines

Off $\quad$ On $\quad$ Problem

$16: 45$

$17: 30 \quad 18: 30$

$22: 10 \quad 22: 30$

$03: 10 \quad 03: 30$

$03: 35$

$09: 25$

$11: 30$

$10: 45$

$11: 45$

$17: 45$

$22: 10$

$09: 45 \quad 07: 40$

\section{$x$}

$\mathrm{x}$

$18: 00$

$13: 08$

$15: 00$

$16: 10$

$16: 15$

$19: 00$

$12: 35$

$12: 55$

$12: 00$

$12: 55$

$13: 30$

$11: 45 . \quad 15: 40$

$15: 40$

$17: 10$

$10: 35$

$13: 50$

$13: 55$

$17: 10$

$13: 40$

$23: 07$
Not recorded.

Gas engines $O K$.

Lost cooling water.

Low instrument air.

Low instrument air;

freezing.

Instrument air closed down due to freezing conditions. Gas engines continued to run.

Grid.

Mustang repair.

Mustang repair.

Bentley Nevada probe test.

Tripped turbine twice for Bentley Nevada test.

Time not logged.

GE-1 down from 19:00

until 12-30 \& 13:00.

GE-2 down 21:00 Test.

GE-1E Re-running Test 4A.

GE-2.

GE-1E not running right.

Replaced magneto.

GE-1 Mustang repair;

oil change.

GE-2.

GE-1 Mustang repair value adjustment.

GE-2 " "

Not running right.

G - 4 


\section{PLANT OUTAGES}

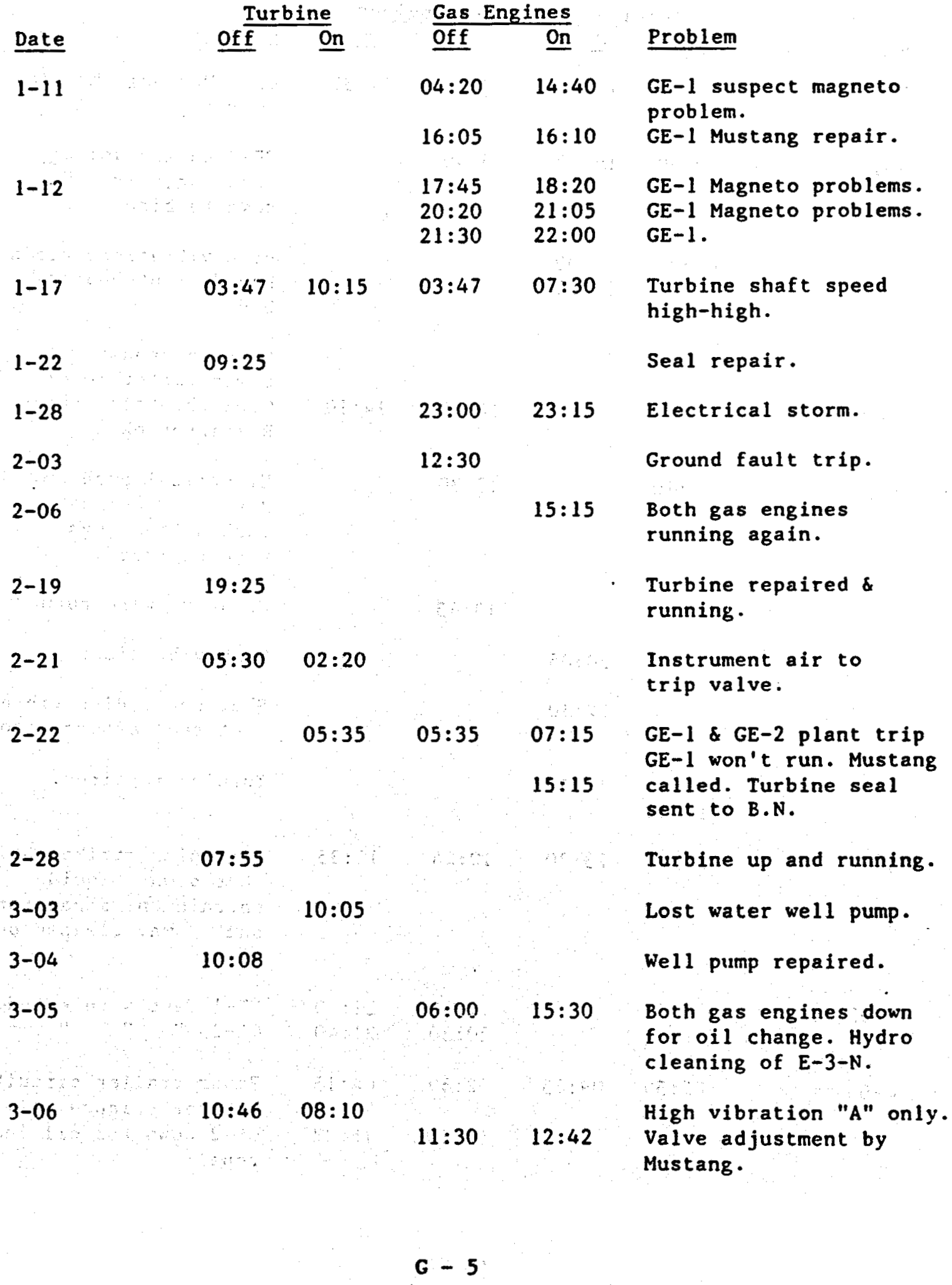




\section{PLANT OUTACES}

\begin{tabular}{|c|c|c|c|c|c|}
\hline \multirow[b]{2}{*}{ Date } & \multicolumn{2}{|c|}{ Turbine } & \multicolumn{2}{|c|}{ Gas Engines } & \multirow[b]{2}{*}{ Problem } \\
\hline & $\underline{\text { of } \bar{f}}$ & On & off & $\underline{\text { On }}$ & \\
\hline $3-07$ & & $10: 25$ & $17: 19$ & $17: 39$ & $\begin{array}{l}\text { GE-2 Mustang checking } \\
\text { engine. }\end{array}$ \\
\hline $3-12$ & $20: 50$ & $18: 40$ & $18: 40$ & & $\begin{array}{l}\text { GE-2 engine voltage } \\
\text { bouncing; also shut } \\
\text { down turbine. }\end{array}$ \\
\hline $3-13$ & $09: 30$ & $\begin{array}{l}09: 00 \\
09: 50\end{array}$ & & & $\begin{array}{l}\text { High vibrations again. } \\
\text { Found front bearing } \\
\text { bad. }\end{array}$ \\
\hline $3-14$ & & & & & $\begin{array}{l}\text { Mustang repair } \\
\text { power factor meter. }\end{array}$ \\
\hline $3-19$ & & & $14: 00$ & $14: 10$ & $\begin{array}{l}\text { GE-2 throttle stuck- } \\
\text { Restarted ok. }\end{array}$ \\
\hline $3-21$ & $18: 10$ & & $15: 30$ & & $\begin{array}{l}\text { Electrical problem. } \\
\text { Both gas engines } \\
\text { turbine bearings } \\
\text { were replaced. }\end{array}$ \\
\hline $3-22$ & & & $13: 45$ & & Negative wire hurned. \\
\hline $3-23$ & & $20: 05$ & & & High vibrations. \\
\hline & & $22: 10$ & & & $\begin{array}{l}\text { Shut down. High vibrations; } \\
\text { sent seal assembly to B.N. }\end{array}$ \\
\hline $3-29$ & $\begin{array}{l}13: 50 \\
16: 22\end{array}$ & $15: 50$ & & & Turbine repaired. \\
\hline $3-30$ & $10: 24$ & $23: 30$ & $10: 24$ & $11: 35$ & $\begin{array}{l}\text { Lightning strike; Grid } \\
\text { shut down. Liquid } \\
\text { entrainment separator } \\
\text { shut down. Cleaned out } \\
\text { sand. }\end{array}$ \\
\hline $4-02$ & & & $\begin{array}{l}17: 30 \\
20: 30\end{array}$ & $\begin{array}{l}21: 40 \\
21: 40\end{array}$ & $\begin{array}{lclc}\text { GE-1 } & \text { Test } & 4 & \text { repeated. } \\
\text { GE-2 " } & \text { " }\end{array}$ \\
\hline $4-03$ & $02: 59$ & $09: 45$ & $02: 59$ & $\begin{array}{l}04: 15 \\
16: 32\end{array}$ & $\begin{array}{l}\text { Power trailer circuit } \\
\text { breaker tripped. } \\
\text { GE-2 down for oil leak } \\
\text { repair. }\end{array}$ \\
\hline
\end{tabular}


PLANT OUTAGES

\begin{tabular}{|c|c|c|c|c|c|}
\hline \multirow[b]{2}{*}{ Date } & \multicolumn{2}{|c|}{ Turbine } & \multicolumn{2}{|c|}{ Gas Engines } & \multirow[b]{2}{*}{ Problem } \\
\hline & Off & $\underline{\underline{0}}$ & Off & $\underline{\underline{O n}}$ & \\
\hline $4-05$ & & & $06: 30$ & & GE-1 electrical problem. \\
\hline $4-06$ & $16: 30$ & $\begin{array}{l}12: 05 \\
17: 45\end{array}$ & $\begin{array}{l}12: 05 \\
16: 30\end{array}$ & $\begin{array}{l}13: 35 \\
16: 45\end{array}$ & $\begin{array}{l}\text { Grid outage. } \\
\text { Electrical trip grid. }\end{array}$ \\
\hline $4-08$ & $10: 10$ & $13: 40$ & $10: 10$ & $11: 30$ & Grid Shutdown. \\
\hline $4-09$ & $\begin{array}{l}05: 00 \\
16: 25\end{array}$ & $\begin{array}{l}07: 30 \\
21: 10\end{array}$ & & & $\begin{array}{c}\text { Water in oil. } \\
" \| "\end{array}$ \\
\hline $4-10$ & $13: 50$ & $18: 15$ & $13: 50$ & $14: 30$ & Lightning. \\
\hline $4-12$ & $19: 20$ & $20: 05$ & & & $\begin{array}{l}\text { Suspect instrument } \\
\text { air supply. }\end{array}$ \\
\hline $4-17$ & $11: 50$ & & & & Leak in B condenser. \\
\hline $4-19$ & & $08: 10$ & & & \\
\hline $4-22$ & $11: 30$ & $13: 30$ & & & Generator speed low. \\
\hline $4-24$ & & & $\begin{array}{l}09: 30 \\
13: 00\end{array}$ & $\begin{array}{l}12: 00 \\
15: 15\end{array}$ & $\underset{\|}{\text { Mustang uil change. }}$ \\
\hline $4-27$ & & & $\begin{array}{l}11: 30 \\
16: 00 \\
23: 15\end{array}$ & $\begin{array}{l}13: 45 \\
19: 05\end{array}$ & $\begin{array}{l}\text { GE-2 Mustang working. } \\
" \text { " } \\
\text { GE-1 generator ground; } \\
\text { Mustang working on it. }\end{array}$ \\
\hline $4-28$ & & & $12: 30$ & $13: 00$ & $\begin{array}{l}\text { Under voltage trip; } \\
\text { reset. }\end{array}$ \\
\hline $5-04$ & & & & $18: 50$ & GE-1 generator repaired. \\
\hline $5-06$ & $\begin{array}{l}10: 35 \\
12: 45\end{array}$ & $\begin{array}{l}12: 35 \\
13: 15\end{array}$ & & & $\underset{"}{\operatorname{Generator} \text { speed }}$ low. $^{\text {. }}$ \\
\hline $5-09$ & & & $10: 15$ & $16: 20$ & $\begin{array}{l}\text { Both gas engines shut } \\
\text { down for low exhaust } \\
\text { gas test. }\end{array}$ \\
\hline & & & $14: 00$ & $16: 20$ & \\
\hline $5-22$ & $\begin{array}{l}13: 01 \\
13: 24\end{array}$ & $15: 29$ & $\begin{array}{l}13: 01 \\
13: 24\end{array}$ & $13: 50$ & Lightning strike. \\
\hline
\end{tabular}




\section{PLANT OUTAGES}

\begin{tabular}{|c|c|c|c|c|c|}
\hline \multirow[b]{2}{*}{ Date } & \multicolumn{2}{|c|}{ Turbine } & \multicolumn{2}{|c|}{ Gas Engines } & \multirow[b]{2}{*}{ Problem } \\
\hline & Of $\overline{\mathrm{f}}$ & On & off & $\underline{\text { On }}$ & \\
\hline $5-23$ & & & $05: 30$ & & $\begin{array}{l}\text { GE-1 generator problem } \\
\text { power factor. }\end{array}$ \\
\hline $5-24$ & & & $12: 15$ & $13: 00$ & $\begin{array}{l}\text { Repair regulators by } \\
\text { Mustang. }\end{array}$ \\
\hline $5-26$ & $20: 00$ & & & & $\begin{array}{l}\text { Suspect brine leak in } \\
E-2-N \text {. }\end{array}$ \\
\hline $5-31$ & $07: 00$ & & $07: 00$ & & $\begin{array}{l}\text { Plant shut down. No } \\
\text { brine or natural gas. }\end{array}$ \\
\hline
\end{tabular}

G -8

THE BEN HOLT CO. 
APPENDIX H

PRINCIPAL EQUATIONS FOR PROCESS CALCULATIONS

THE BEN HOLTCO. 


\section{Appendix $\mathrm{H}$}

\section{PRINCIPAL EQUATIONS FOR PROCESS CALCULATIONS}

Heat Exchanger Duty:

$$
Q=M \Delta h
$$

(can be approximated as $Q=M C_{p} \Delta T$ for single-phase fluids) Isobutane enthalpy is calculated from measured pressure and temperature using the Starling equation of state. Brine enthalpy is calculated from the measured pressure and temperature for a $13 \%$ salinity solution using equations from Dittman, "Calculation of Brine Properties".?

Measured Overall Heat Transfer Coefficient:

$$
\begin{aligned}
U_{0, \text { moas }} & =Q / A\left(\Delta T_{m}\right) \\
\Delta T_{L M} & =\frac{\left(T_{n}-t_{n}\right)-\left(T_{c}-t_{d}\right)}{\ln \frac{\left(T_{n}-t_{n}\right)}{\left(T_{c}-t_{c}\right)}}
\end{aligned}
$$

(Note that the LMTD correction factor is unity for all three heat exchangers in this plant: $E-1-N$ is a counter-current exchanger, and E-2-N and E-3-N have an isothermal fluid on the shellside.)

Calculated Overall Heat Transfer Coefficient:

$$
U_{0}=\left[\frac{1}{h_{i}} \times \frac{d_{0}}{d_{i}}+\frac{d_{0}}{2 k} \ln \frac{d_{0}}{d_{i}}+\frac{1}{h_{0}}+f_{i} \times \frac{d_{0}}{d_{i}}+f_{0}\right]^{-1}
$$

$$
\text { H }-1
$$


Film heat transfer coefficients are calculated for the tube and shell side of each exchanger as follows:

Tubeside:

$$
N u=h_{i} d_{i} / k_{i}=0.023 \operatorname{Re}{ }^{0.8} \operatorname{Pr}{ }^{0.3}
$$

This correlation for Nusselt number is recommended for fully developed turbulent flow in smooth tubes. ${ }^{3}$ Brine density was evaluated at the mean fluid temperature using Dittman's correlation. Brine transport properties were evaluated at bulk fluid temperatures using correlations found in Phillips, "A Technical Databook for Geothermal Energy Utilization." 4

Shellside:

$\mathrm{Nu}=h_{0} d_{0} / k_{0}=C \operatorname{Re}^{n} \operatorname{Pr}^{1 / 3}$

heater:

$\mathrm{C}$ and $\mathrm{n}$ are constants from a table in Holman ${ }^{5}$ for flow across tube banks based on tube spacing and pitch

boilers:

$C$ and $n$ are constants evaluated based on correlations in Holman. ${ }^{5}$ Since the heat transfer coefficient for a boiling fluid varies greatly depending on the boiling regime, these coefficients were adjusted so that they fit the overall heat transfer coefficients as supplied by the manufacturer.

Fouling Factor:

$$
f f_{\infty a}=1 / U_{o, \text { meas }}-1 / U_{0, \text { calc }}
$$


Nomenclature:

A Area (based on outside surface), sq. ft.

$C_{p}$ Heat capacity at constant pressure, Btu/lb - ${ }^{\circ} \mathrm{F}$

d Inside diameter, inches

d. Outside diameter, inches

$f_{f} \quad$ Inside fouling factor, $h r$ - sq $\mathrm{ft}$ - ${ }^{\circ} \mathrm{F} / \mathrm{Btu}$

$f f_{0} \quad$ Outside fouling factor, $\mathrm{hr}-\mathrm{sq} \mathrm{ft}-{ }^{\circ} \mathrm{F} / \mathrm{Btu}$

$\mathrm{ff}_{\mathrm{oA}}$ Overall fouling factor, $\mathrm{hr}-\mathrm{sq} \mathrm{ft}-{ }^{\circ} \mathrm{F} / \mathrm{Btu}$

$h_{b}$ Inside film heat transfer coefficient, Btu/hr - sq ft - ${ }^{\circ} \mathrm{F}$

$h_{0}$ Outside film heat transfer coefficient, Btu/hr - sq ft - ${ }^{\circ} \mathrm{F}$

$\Delta h \quad$ Enthalpy change of fluid through a heat exchanger, Btu/lb

$k$ Thermal conductivity of tube metal, Btu/hr - $\mathrm{ft}$ - ${ }^{\circ} \mathrm{F}$

$k_{1} \quad$ Thermal conductivity of fluid inside tubes, Btu/hr - $\mathrm{ft}-{ }^{\circ} \mathrm{F}$

$k_{\circ}$ Thermal conductivity of fluid outside tubes, Btu/hr - ft $-^{\circ} \mathrm{F}$

$M$ Mass flow rate, lb/hr

$\mathrm{Nu}$ Nusselt number, dimensionless

Pr Prandtl number, dimensionless

Q Duty, Btu/hr

Re Reynolds number, dimensionless

$T_{c}$ Temperature of hot fluid leaving heat exchanger, ${ }^{\circ} \mathrm{F}$

$t$. Temperature of cold fluid entering heat exchanger, ${ }^{\circ} \mathrm{F}$

$T_{n} \quad$ Temperature of hot fluid entering heat exchanger, ${ }^{\circ} \mathrm{F}$

$t_{n}$ Temperature of cold fluid leaving heat exchanger, ${ }^{\circ} \mathrm{F}$

$\Delta T$ Temperature change of a fluid in a heat exchanger, ${ }^{\circ} \mathrm{F}$

$\Delta T_{w}$ Logarithmic mean temperature difference (LMTD), ${ }^{\circ} \mathrm{F}$

$U_{o, m e a s}$ Measured overall heat transfer coefficient (based on outside surface area), Btu/hr - sq $\mathrm{ft}$ - ${ }^{\circ} \mathrm{F}$

$U_{\text {o.calc }}$ Calculated overall heat transfer coefficient (based on outside surface area), Btu/hr - sq ft - of 
References:

1. Starling, K., Fluid Thermodynamic Properties for Light Petroleum Systems, Gulf Publishing Co., Houston, 1973.

2. Dittman, G., "Calculation of Brine Properties", Lawrence Livermore Laboratory, UCID-17406, February 1977.

3. Holman, Heat Transfer, McGraw-Hill, New York, 1981, pp. 226-227.

4. Phillips, S., "A Technical Databook for Geothermal Energy Utilization", Lawrence-Berkeley Laboratory, LBL-12810, June 1981. 


\section{APPENDIX 1}

\section{COMPUTER PROGRAM AND SAMPLE OUTPUT}

\section{PROGRAM WRITTEN IN MICROSOFT QUICK BASIC VERSION 1.0}

FOR MACINTOSH SE 
DIM $\quad k(20), G(20), H(20), c(6), V(6), R(6), P(6), U(6), W(6), M(20), a(6), B(6), E(21)$

REM K-TEMP, G-PRESS, H-ENTHALPY, M-FLOW, C-TH CONDUCTIVITY

REM V-VISCOSITY, R-RE NUMBER,P-SKIN EEAT TRANS

REM U-OVERALL HEAT TRANSFER COEFF, W-FOULING FACTOR

REM SAMPLE TEMP, PRESS AND FLOW ENTERED

IPRINT "RUN DATE IS ",DATES

OREN "CLIP:" FOR INPUT AS \#1

INPUT \#1,DATE1\$

INPUT \#1, TIMEIS

FOR $\mathrm{KK}=1$ TO 12:INRPT $\# 1$, DUM:NREXT KK

INPUT \#1, $G(1)$

INPUT \#1, $G(2)$

INPUT \#1, G(3)

INPUT \#1, DUM

INPUT \#1, G(14)

INPUT \#1, G(15)

INPUT \#1, G(16)

INPUT \#1, G(19)

INPUT \#1, G(20)

INPUT \#1, G(11)

INPUT \#1, G(12)

INPUT \#1, GORI

INPUT \#1, GOR2

INPUT \#1, DUM

INPUT \#1, $G(8)$

INPUT \#1, DUM

INPUT \#1, DUM

INPUT \#1, $k(1)$

RRTNT $k(1)$

INPUT \#1, $k(2)$

INPUT \#1, $k(3)$

FOR KK=1 TO 9: INPUT \#1, DUM:NEXT KK

INPUT \#1, $k$ (14)

PRINT k (14)

INPUT \#1, $k$ (15)

INPUT \#1, $k$ (16)

INPUT \#1, $k$ (19)

INPUT \#1, $k(20)$

INPUT \#1, $k$ (II)

INPUT \#1, $\mathrm{k}(12)$

INPUT \#1, DUM

INPUT \#1, $k(8)$

PRINT $k(8)$

INPUT \#1, $k(9)$

INPUT \#I, DUM

INPUT \#1, $M(3)$

PRINT $M(3)$

INPUT \#1, M(15)

INPUT \#1, M(11)

INPUT \#1, M(17)

INPUT \#1, DUM

INPUT \#I, $M(6)$

PRINT $M(6)$

PRINT $G(1)$

PRINT G (12)

PRINT $k(3)$ 


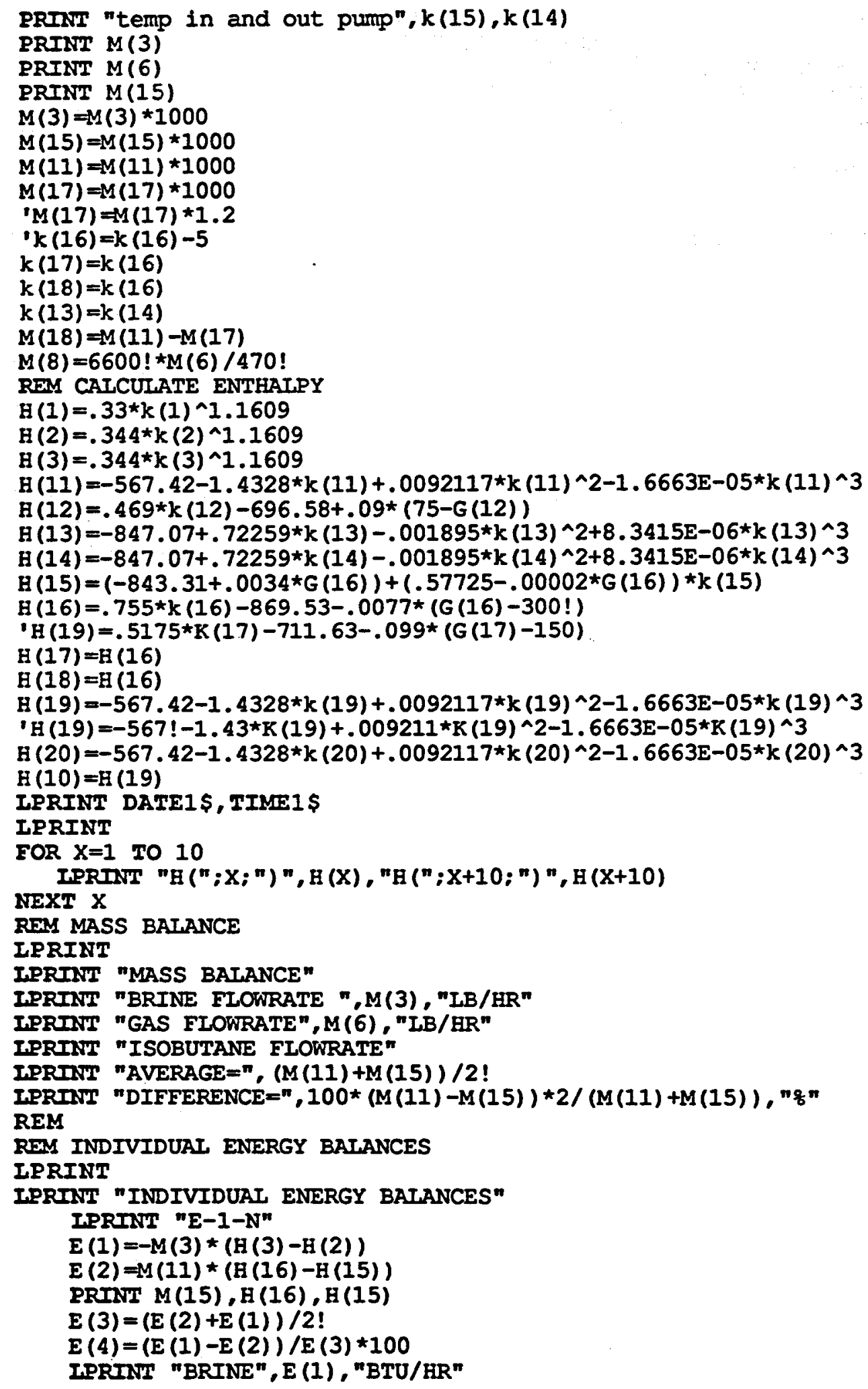


IPRTNT"ISOBUTANE", E (2), "BTU/HR"

IFRTNT "AVERAGE=", $E$ (3)

IPRNNT "DIFFERENCE $=", E(4), n$ "

IPRINTT "E-2-N"

$E(5)=-M(3) *(H(2)-H(1))$

$E(6)=M(18) *(H(19)-H(18))$

$E(7)=(E(5)+E(6)) / 2$ !

$E(8)=(E(5)-E(6)) / E(7) \star 100$

IPRTNT "BRINE", E (5), "BTU/HR"

IPRINT" ISOBUTANE" , E (6), "BTU/HR"

IPRINT "AVERAGE="., E (7)

IPRINI "DIFFERENCE $=", E(8), n$ "

IPRINTT "E-3-N"

IF $M(17)<2000$ THEN

$E(9)=0$ !

$E(10)=0$ !

$E(11)=0$ !

$E(12)=0$ !

ELSE

$E(9)=-M(8) * .24 *(k(9)-k(8))$

$E(10)=M(17) *(B(20)-B(17))$

$E(11)=(E(9)+E(10)) / 2$ !

$E(12)=(E(9)-E(10)) / E(11) * 100$ !

END IF

IPRTNT "EXHAUST", E (9), "BTU/HR"

IPRTNT"ISOBUTANE", E (10), "BTU/HR"

IPRTNT "AVERAGE =", $E$ (11)

IPRTNT "DIFFERENCE =", $E(12), n \% "$

REM OVERALI ENERGY BAIANCE

IPRINT

IPRTNT "OVERALL ENERGY BALANCE"

$E(13)=-M(3) *(B(3)-B(1))$

$E(14)=E(9)$

$E(15)=M(15) *(B(15)-H(14))$

$E(16)=E(13)+E(14)+E(15)$

$E(17)=-M(11) *(B(13)-H(12))$

$E(18)=-M(11)$ * (H (12) $-H(11))$

$E(19)=E(17)+E(18)$

$E(20)=(E(16)+E(19)) / 2$ !

$E(21)=(E(16)-E(19)) / E(20) * 100$ !

IPRINT "ENERGY FROM BRTNE", E (13), "BTU/ER"

IPRTNT "ENERGY FROM EXHAUST",E(14),"BTU/HR

IPRINT "ENERGY EROM PUMP", E (15), "BTU/HR"

IPRTNT "TOTAL ENERGY INTO ISOBUTANE", E (16), "BTU/ER"

IPRNNT "ENERGY TO COOLING WATER", E(17), "BTU/HR"

IPRINT "ENERGY TO TURBINE", E (18), "BTU/ HR" $^{\prime}$

IPRINT "TOTAL ENERGY OUT OE ISOBUTANE",E (19), "BTU/HR"

IPRTNT "AVERAGE",E (20), "BTU/HR"

IPRTNT "DIFEERENCE", E (21), "q"

REM E CALCULATIONS FOR E-I-N

REM

REM TUBE SIDE BRINE

RDM PRORERTY CALCULATION

$a(1)=((k(2)+k(3)) / 2 !-32) / 1.8$

$v(1)=1.0613+.0004309 * a(1)$

$D=(a(1)+273.15) / 273.15$ 
$D=-.92247+2.8395 * D-1.8007 * D^{\wedge} 2+.52557 \star D^{\wedge} 3-.07344 * D^{\wedge} 4$

$C(1)=(.9713+9.963 E-05 \star a(1)-5.1032 E-07 \star a(1) \wedge 2) \star D \star .5778$

$R(1)=.0729 * M(3) / V(1)$

$P(1)=.6887 \star C(1) \star R(1) \wedge .8$

REM - SHELLSIDE ISOBUTANE FOR E-1-N

$a(2)=(k(15)+k(16)) / 2$ !

$v(2)=.5484-.0015073 * a(2)$

$c(2)=.0737-1.7153 E-04 * a(2)$

$R(2)=M(15) / V(2) * .2$

$P(2)=10.11 * C(2) \star R(2) \wedge .569$

REM - E-2-N

REM - TUBESIDE BRINE

$a(3)=((k(2)+k(1)) / 2 !-32) / 1.8$

$v(3)=1.0613+.0004309 * a(3)$

$D=(a(3)+273.15) / 273.15$

$D=-.92247+2.8395 * D-1.8007 * D^{\wedge} 2+.52557 * D^{\wedge} 3-.07344 * D^{\wedge} 4$

$c(3)=(.9713+9.963 E-05 * a(3)-5.1032 E-07 * a(3) \wedge 2) * D * .5778$

$R(3)=.1119 * M(3) / V(3)$

$P(3)=.5014 * C(3) \star R(3) \wedge .8$

REM - SHELISIDE ISOBUTANE

$R(4)=M(18) \star 2.8606$

$P(4)=.4 \star R(4) \wedge .556$

REM - E-3-N

REM - TUBESIDE EXHAUST

$R(5)=M(8) * 3.3956$

$P(5)=.00732 * R(5) \wedge .8$

REM - SHELLSIDE ISOBUTANE

$R(6)=42.94 \times M(17)$

$P(6)=.146 * R(6) \wedge .54$

IPRINT

ITRINT $n$

$\mathbf{R E}$

$\mathrm{h}^{\text {" }}$

FOR $X=1$ TO 6

IPRINT $X, R(X), P(X)$

NEXT $X$

IPRINT

REM CALCULATE U

REM B-IOG MEAN TEMPERATURE DIFFERENCE

PRDNT $k(2), k(16), k(3), k(15)$

$B(1)=((k(2)-k(16))-(k(3)-k(15))) / \operatorname{IOG}((k(2)-k(16)) /(k(3)-k(15)))$

PRTNT $k(1), k(19), k(2), k(18)$

$B(2)=((k(1)-k(19))-(k(2)-k(18))) / \operatorname{IOG}((k(1)-k(19))) /(k(2)-k(18)))$

$B(3)=((k(8)-k(20))-(k(9)-k(17))) / I O G((k(8)-k(20)) /(k(9)-k(17)))$

REM U CALCULATED

$U(4)=1 /(1.2615 / P(1)+.0002413+1 / P(2))$

$U(5)=1 /(1.0832 / P(3)+9.2488 E-05+1 / P(4))$

$U(6)=1 /(1.0854 / P(5)+1.5816 E-04+1 / P(6))$

REM U MEASURED

- $U(1)=e(3) / 2728 / B(1)$

$U(2)=E(7) / 1738 / B(2)$

- base on brine

$U(1)=E(1) / 2728 / B(1)$

- $U(2)=e(5) / 1738 / B(2)$

$U(3)=E(9) / 237 / B(3)$

REM FOULING FACTOR

$W(1)=1 / U(1)-1 / U(4)$ 
$W(2)=1 / U(2)-1 / U(5)$

$W(3)=1 / U(3)-1 / U(6)$

IFRDNT "E-X-N", "UMEAS", "UCALC", "LMTD", "Fouling Factor"

FOR $X=1$ TO 3

IPRINT $X, U(X), U(X+3), B(X), W(X)$

NEXT $X$

PRINT "X", "UMEAS", "UCALC", "DTIM", "FF"

FOR $X=1$ TO 3

PRTNT $X, U(X), U(X+3), B(x), W(X)$

NEXT $X$

STOP 


\begin{tabular}{|c|c|c|c|}
\hline $\begin{array}{l}\text { PUUl DATE IS } \\
5.41000\end{array}$ & $\begin{array}{l}00-25-1000 \\
100\end{array}$ & & \\
\hline 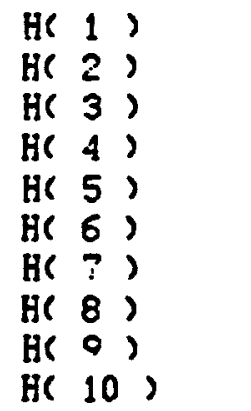 & $\begin{array}{l}245.0414 \\
177.4004 \\
114.6596 \\
0 \\
0 \\
0 \\
0 \\
0 \\
0 \\
-614.4190\end{array}$ & $\begin{array}{l}\text { H( } 11) \\
\text { H( } \\
\text { H( } 12) \\
\text { H( } 14) \\
\text { H( } 15) \\
\text { H( } 16) \\
\text { H( } 17) \\
\text { H( } 18) \\
\text { H( } 19) \\
\text { H( } 20)\end{array}$ & $\begin{array}{l}-615.2643 \\
-631.844 \\
-790.7794 \\
-790.7794 \\
-789.3196 \\
-709.5471 \\
-709.5471 \\
-709.5471 \\
-614.4199 \\
-615.9301\end{array}$ \\
\hline
\end{tabular}

\section{MASS BALANCE}

BRIHE FLOWRATE

GAS FLOWRATE 493.4
ISOBUTANE FLOWRATE

$149100 \quad$ LB/HR

AVERAGE = 120800

DIFFERENCE $=2.317881$

IHDIVIDUAL ENERGY BALANCES

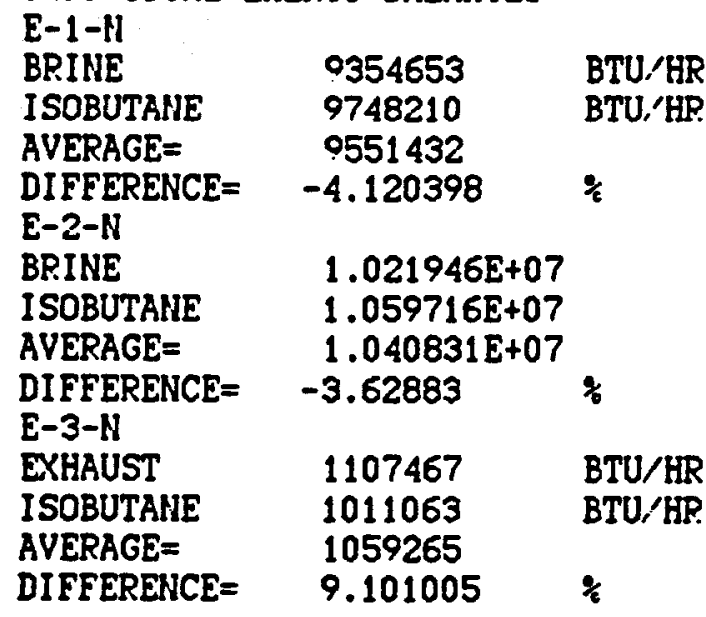

OVERALL ENERGY BALANCE

ENERGY FROM BRINE

ENER.GY FROM EXHAUST

ENERGY FROM PUMP

$1.957412 E+07$

1107467 BTU/HP

$174297.5 \quad \mathrm{BTU} / \mathrm{HR}$

TOTAL ENERGY INTO ISOBUTANE

ENERGY TO COOLING WATER

ENERGY TO TURBINE

$2.085588 \mathrm{E}+07$

$1.942191 \mathrm{E}+07$

2026041

BTU/HR

BTU/HR

TOTAL ENERGY OUT OF ISOBUTANE

\section{AVERAGE \\ DIFFERERCE \\ $2.115191 \mathrm{E}+0$ ? \\ $-2.799123 \%$}

BTU/HR
BTU/HR

BTU, /HP.

2.144795E+07

BTU/HP

BTU/HR

BTU/HR

BTU/HR

h

410.4125

285.5545

424.6619

459.1116

23.00173

167.5511

UMEAS

UCALC

146.6922

207.4111

12.71399

18.75672

LMTD

Foullng Factor

$26.8115 \quad-3.443076 \mathrm{E}-04$

367.5365 


\section{APPENDIX J}

\section{CHEMICAL ANALYSIS, SCANNING ELECTRON}

\section{MICROSCOPY AND LUBE OIL ANALYSIS RESULTS}


Report No.: $\quad 6971$

Prepared For:
Ms. Mai Hattar

Ben Holt Company

201 S. Lake Ave. Suite 308

Pasadena, CA 91101
Date: July 12, 1990

P.O. No.: $30008-258$

\section{PURPOSE OF ANALYSIS:}

To elementally characterize the deposits on the inside and outside surfaces of six tubes. Also, to determine the minimum remaining wall thickness of each tube.

\section{SAMIPLES:}

Six (6) tubes:

A:E-2-N, 4th pass tube.

B:E-2-N, tube \#2, 1st pass.

C: $E-3-N$, tube \#6.

D:E-2-N, ferrule on 1st pass inlet.

E:E-2-N, ferrule on 5 th pass inlet.

$\mathrm{F}:$ Condenser tube.

\section{METHODS OF ANALYSIS:}

Scanning Electron Microscopy (SEM).

Energy Dispersive X-ray Spectroscopy (EDS).

\section{RESULTS:}

The material and operating environments for the tubes was reported as follows.

E-2-N

E-3-N

Condenser :geothermal brine inside tube. :isobutane outside tube. :carbon steel.

:engine exhaust gas inside tube (fuel:natural gas). :isobutane outside tube. :304 S.S..

:isobutane inside tube. :water and air outside tube. 
The inside (I.D.) and outside (O.D.) of each tube was analyzed with EDS to determine the elemental nature of the corrosion product/deposit (see spectra 1 through 12).

A sample of tube wall from each sample was encapsulated in clear epoxy and cross-sectioned using standard metallographic techniques. The samples were gold coated for SEM documentation of the minimum tube wall thickness. The dimensions can be seen in table 1.

The enclosed micrographs and spectra further present the results of this analysis.

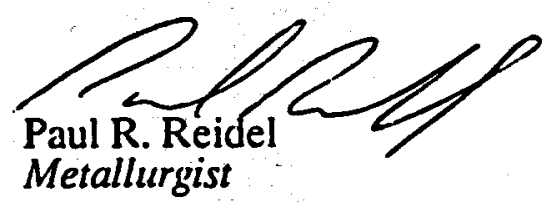

PRR:cp

$\begin{array}{lll}\text { Enclosures: } & \text { Samples } & (6) \\ & \text { Micrographs } & (6) \\ & \text { Spectra } & (12)\end{array}$


PAGE 3 OF 18

\section{D7ר PhotoMetrics}

Table 1

Sample Minimum Remaining Wall Thickness

A
B
D
E

0.074 inches

0.074 inches

0.069 inches

0.013 inches

0.035 inches

0.054 inches 


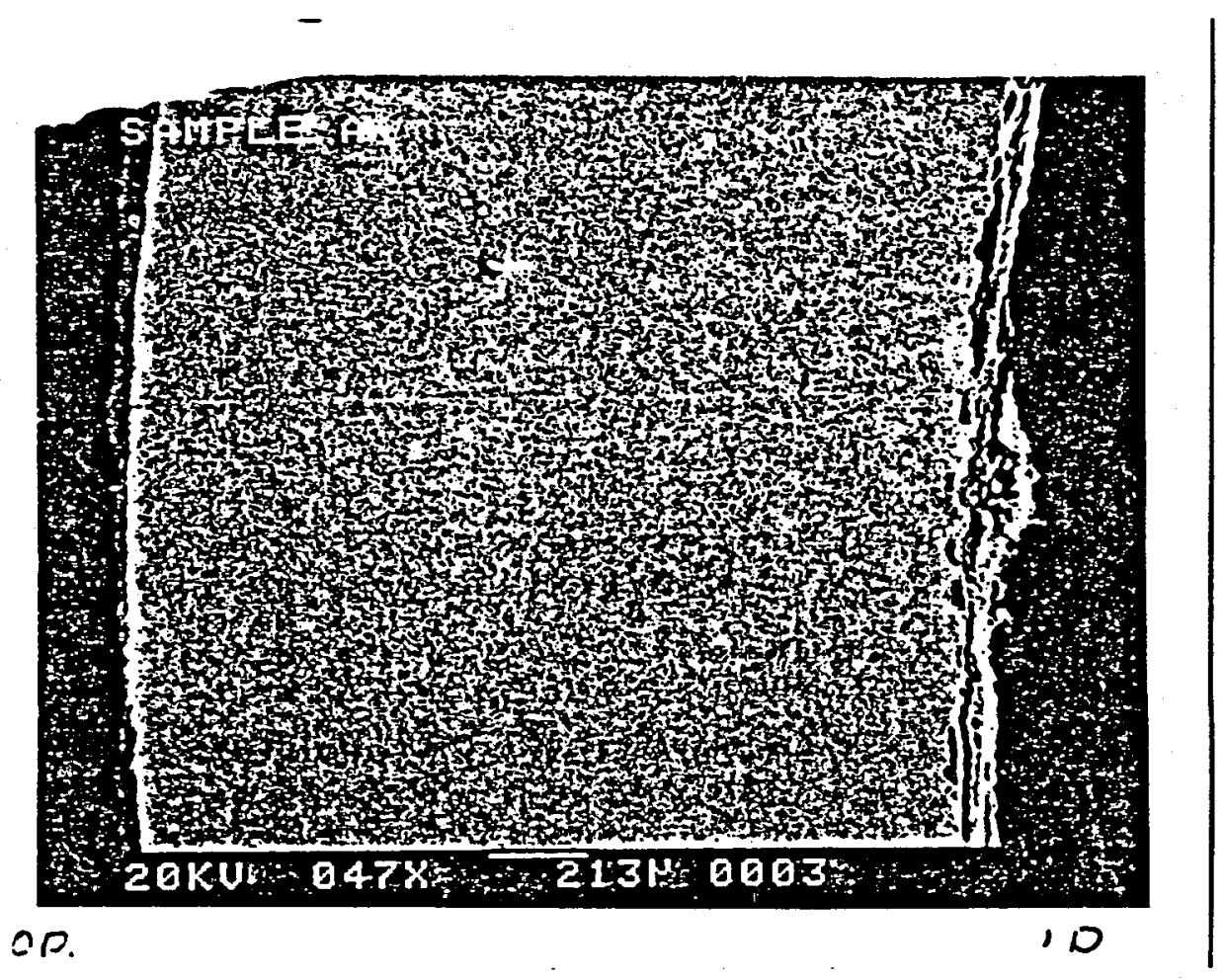

$M-1$

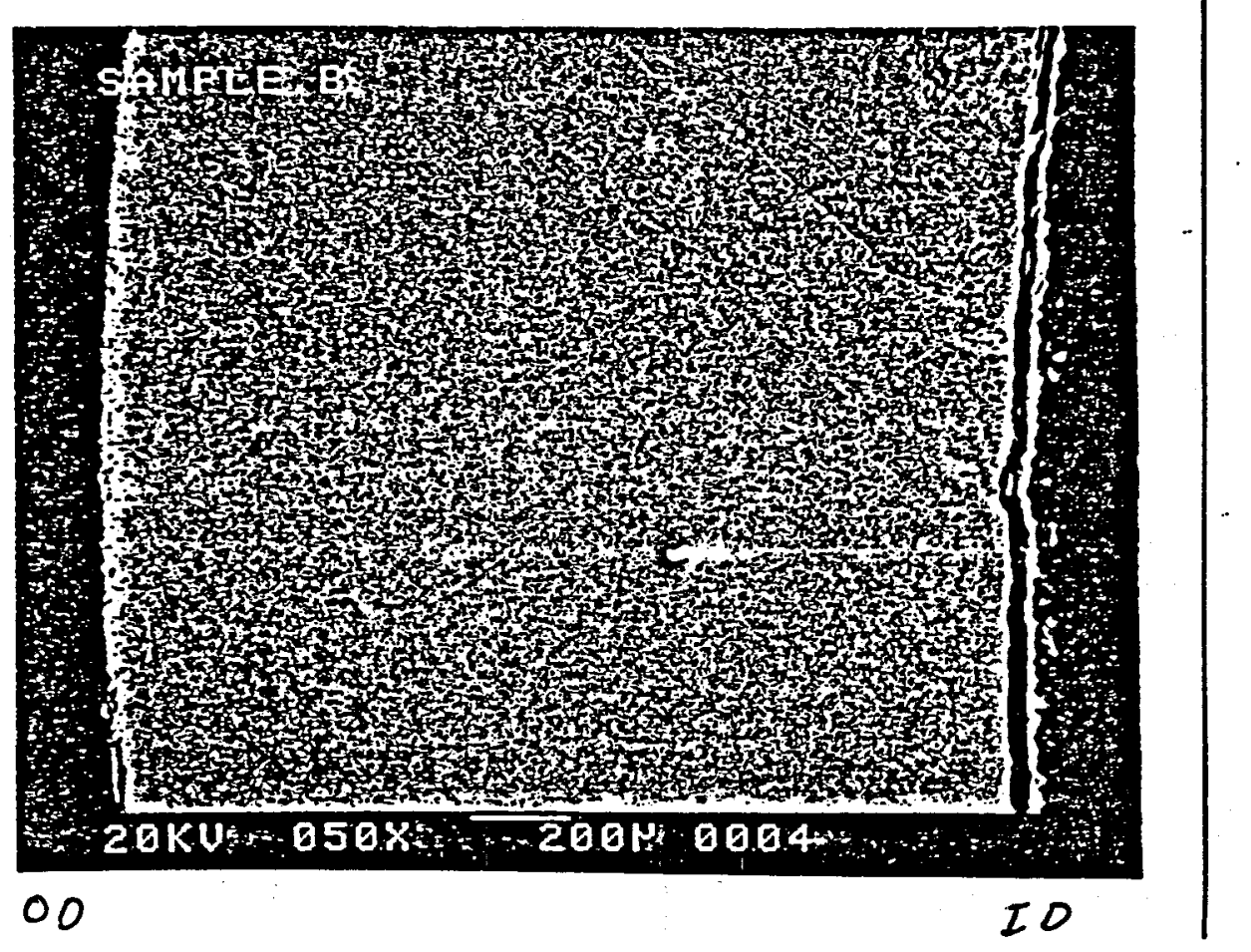

$M-2$

J. 4 
PAGE 5 OF 18

Diר PhotoMetrics
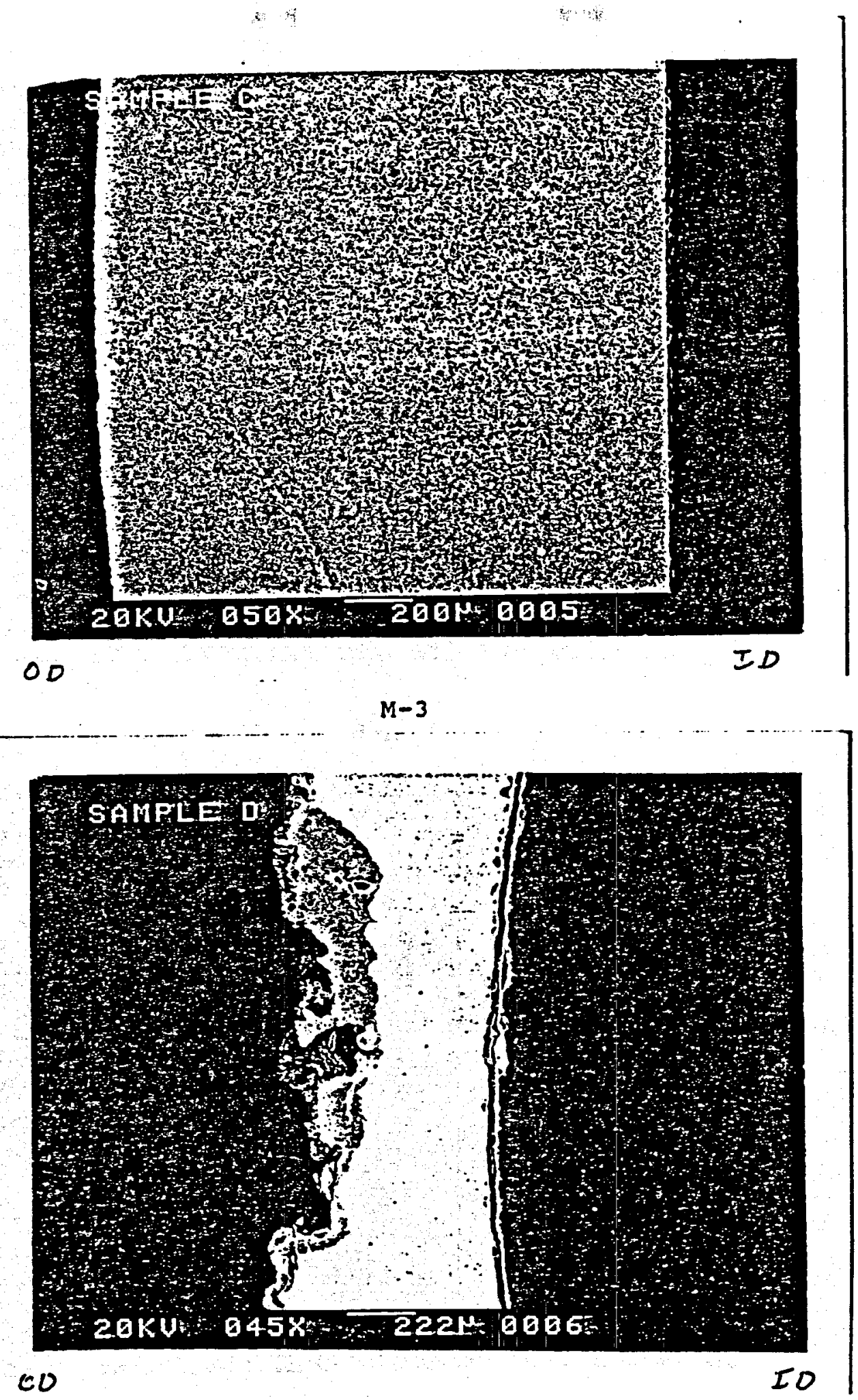

$$
\begin{aligned}
& M-4 \\
& J-5
\end{aligned}
$$


Di] PhotoMetrics

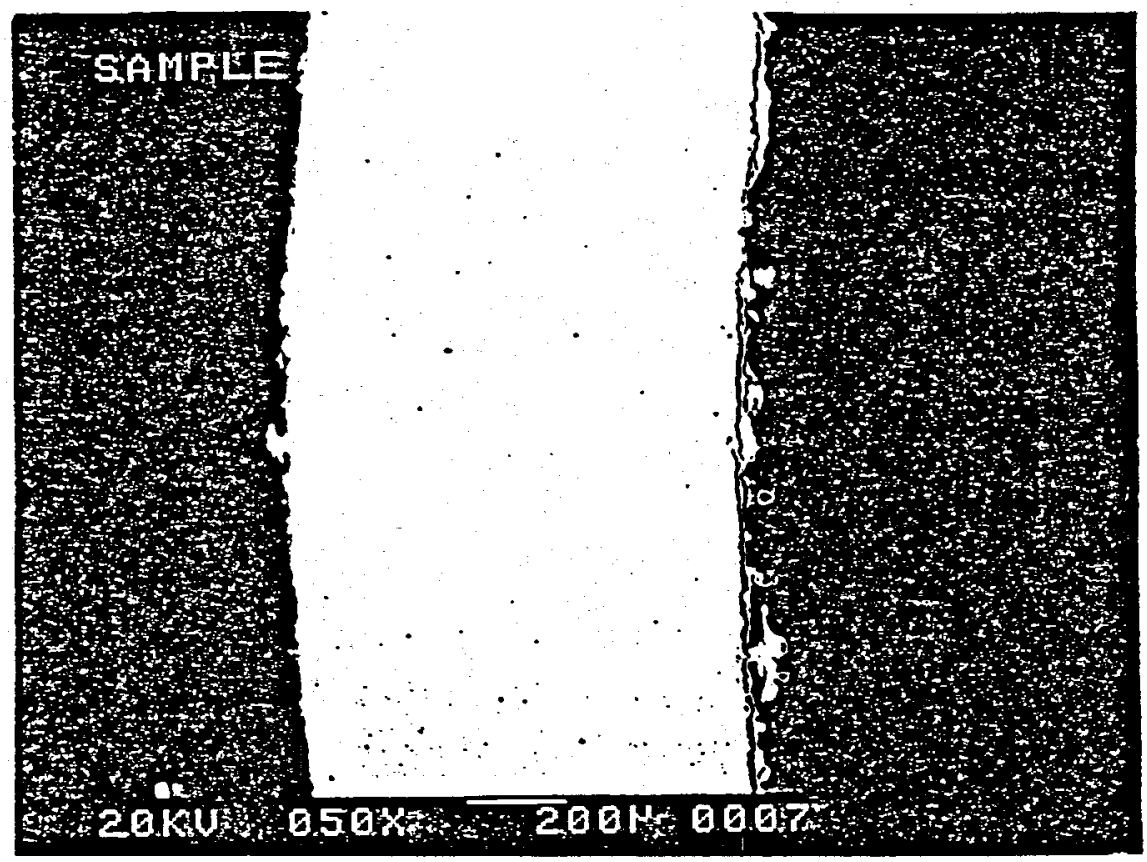

$M-5$

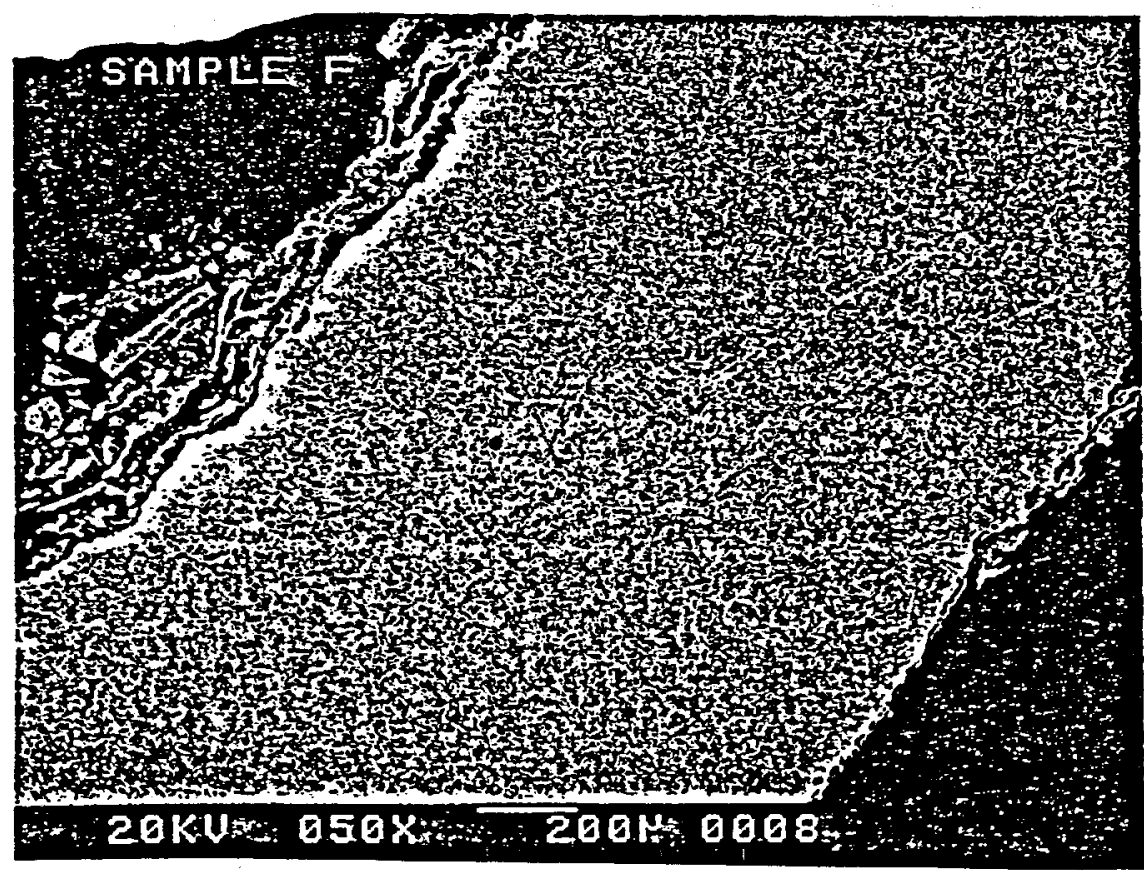

$M-6$

J. 6 


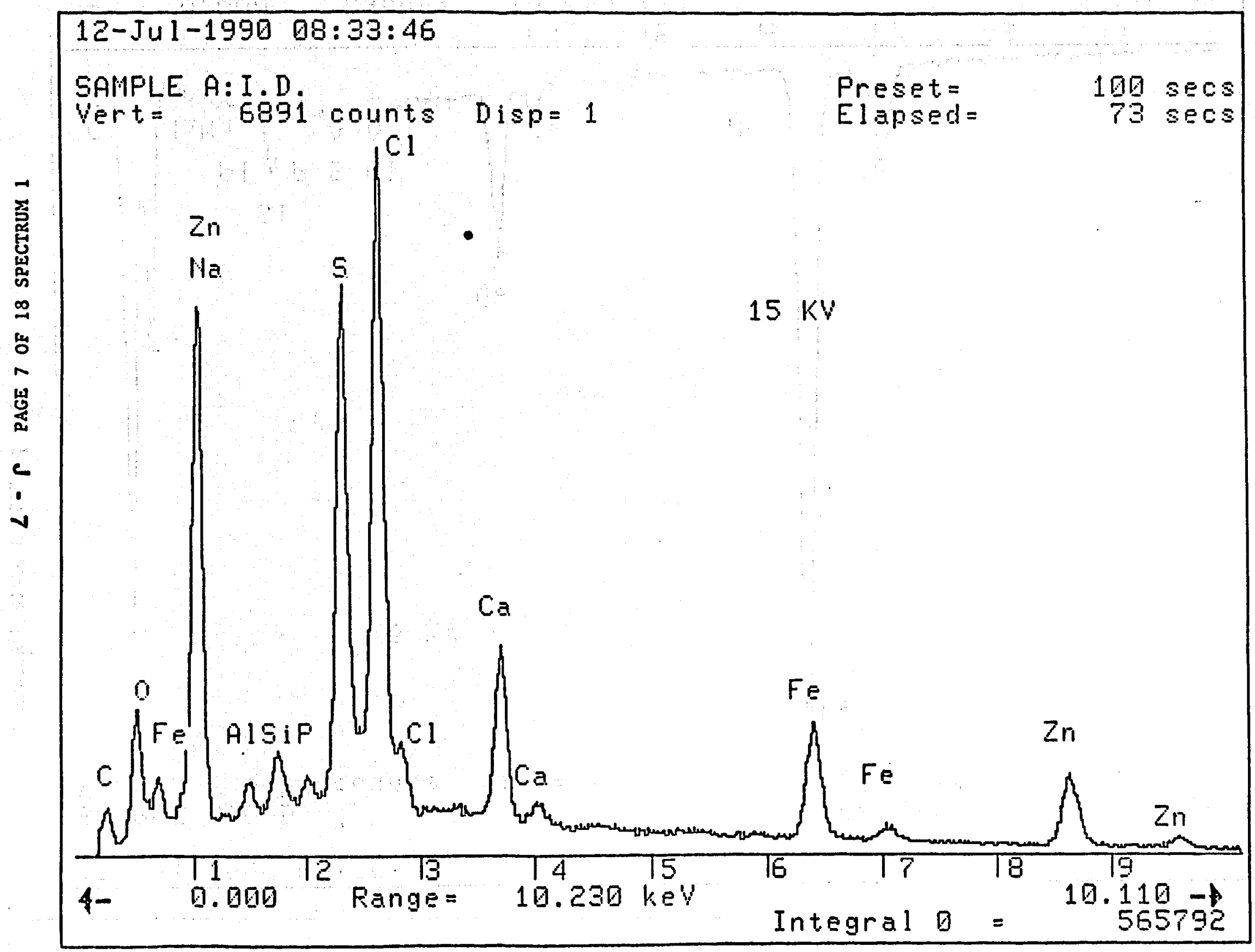




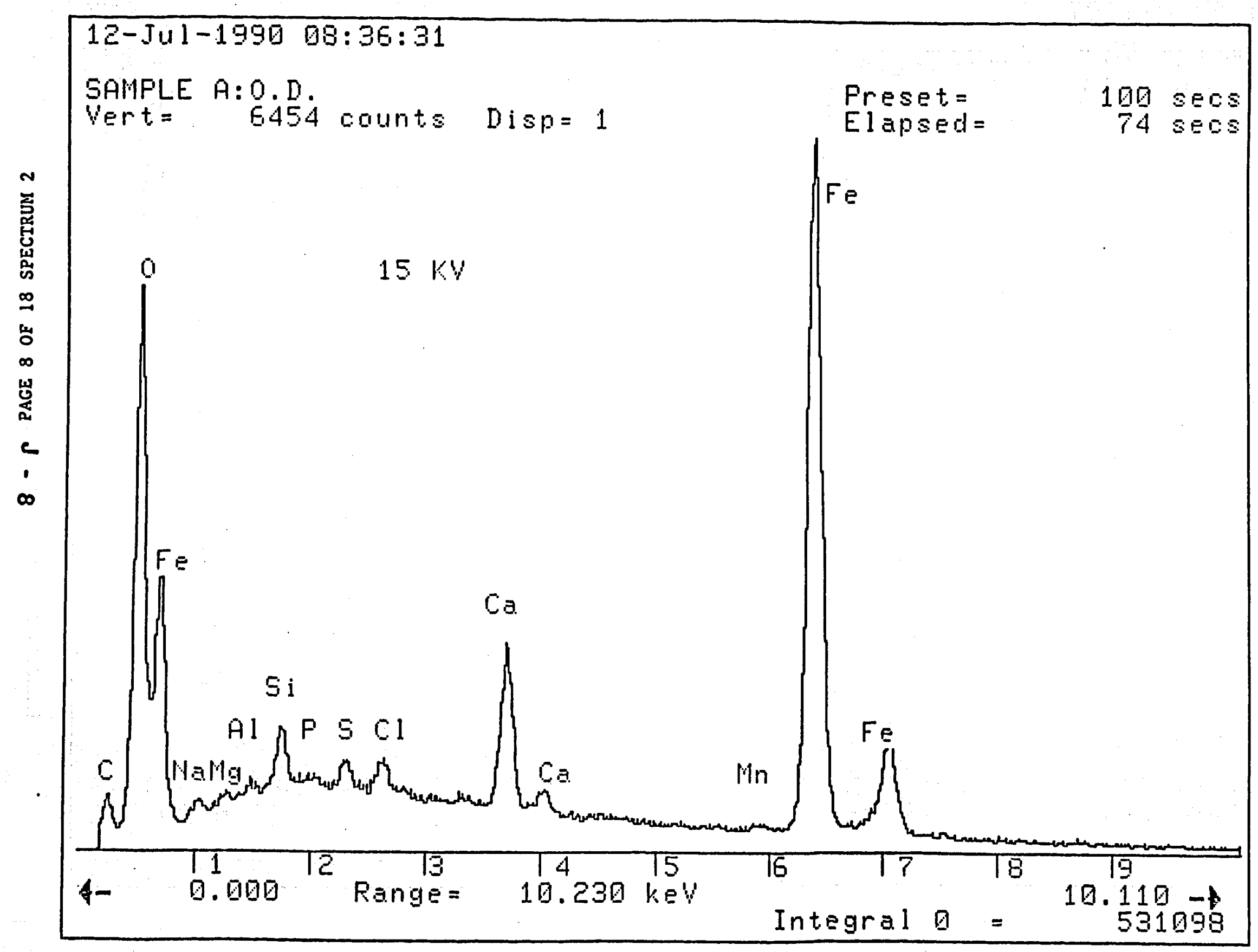




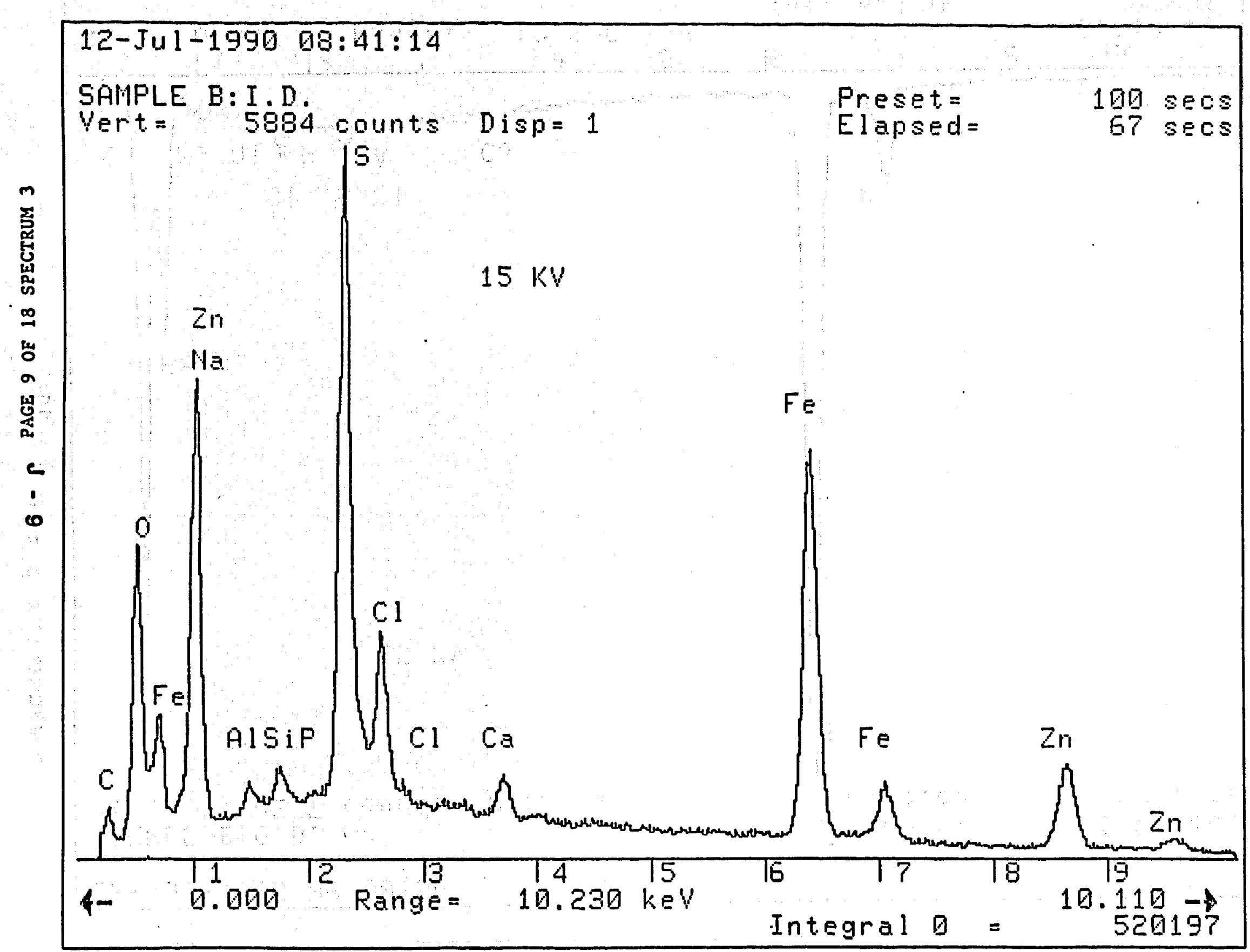




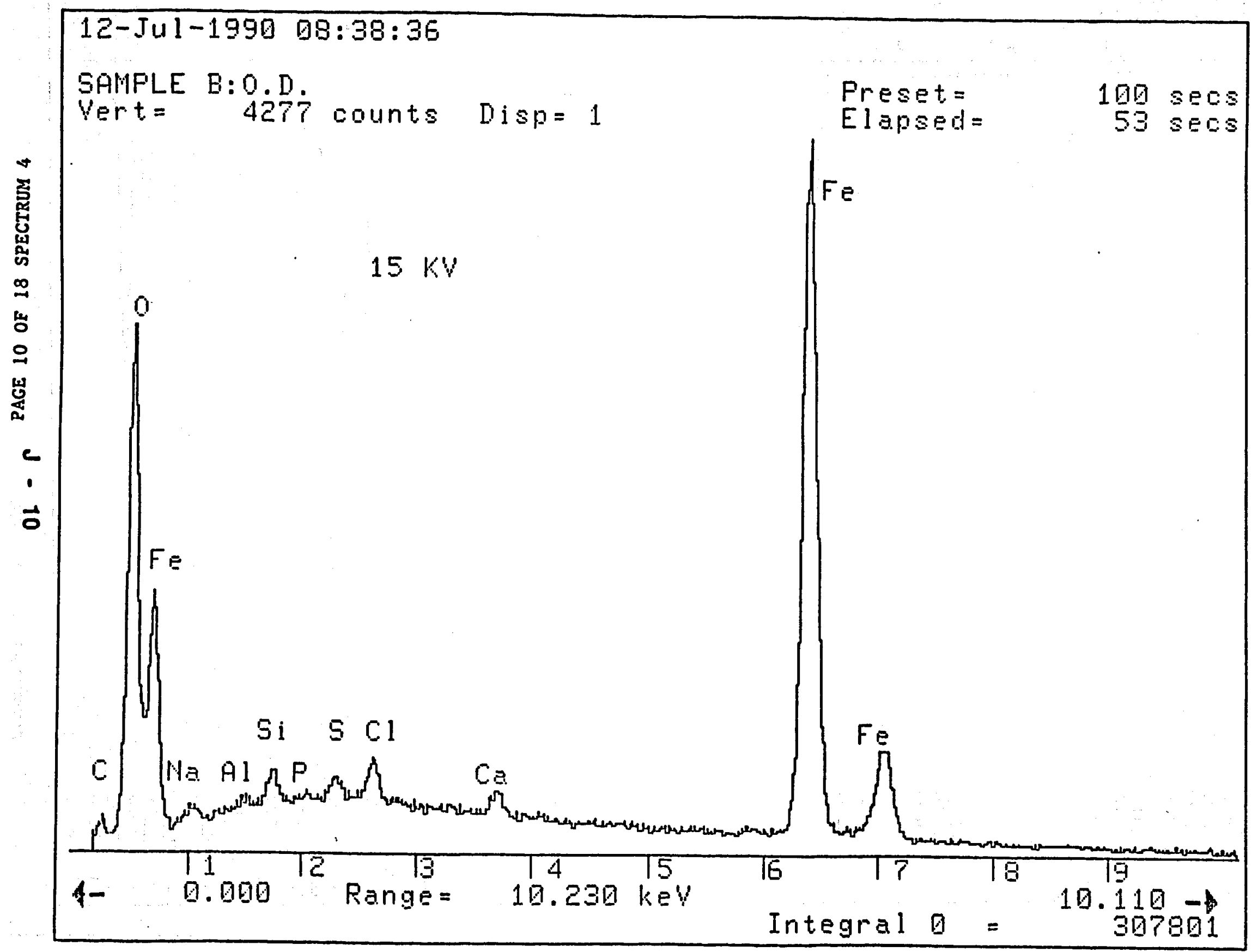




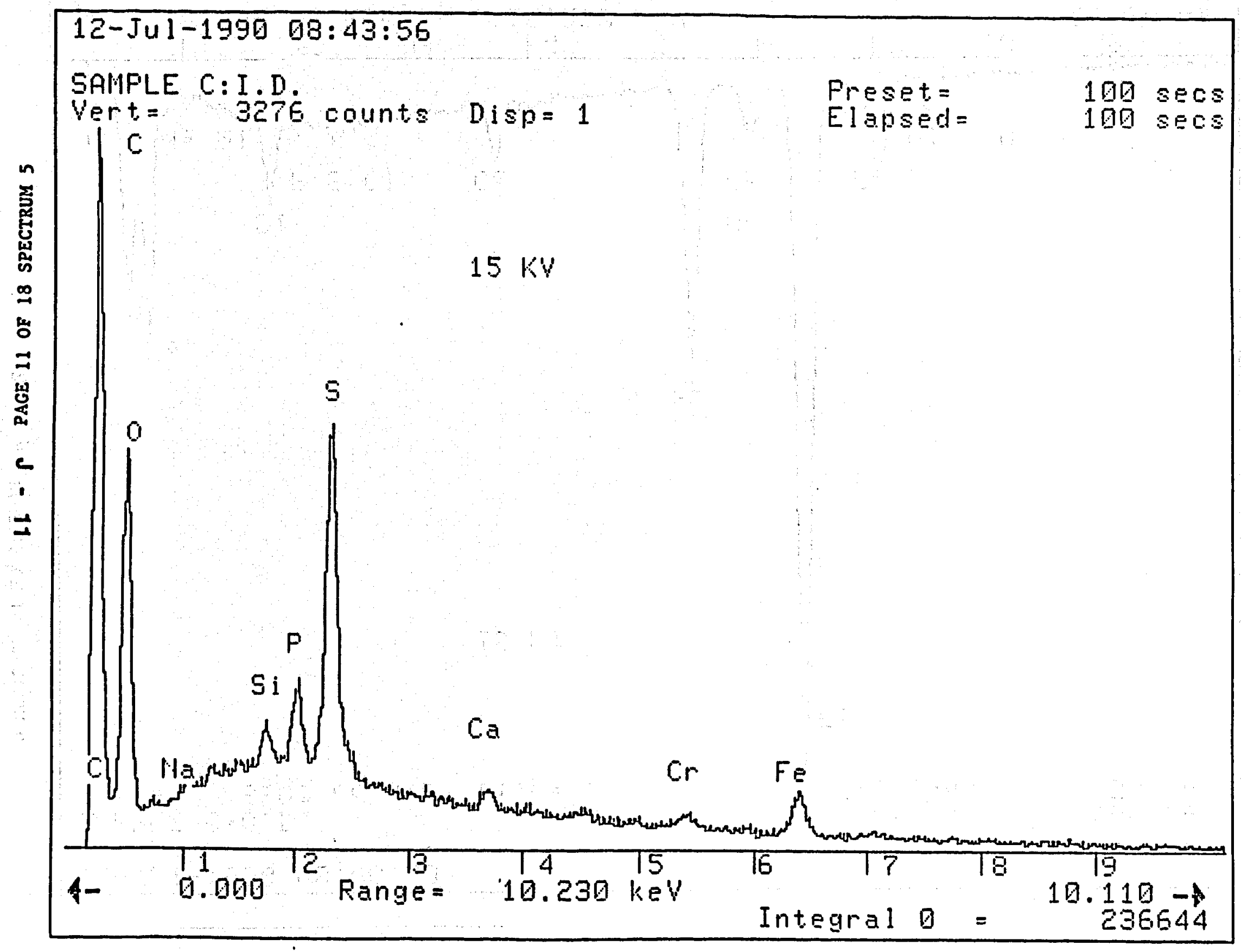




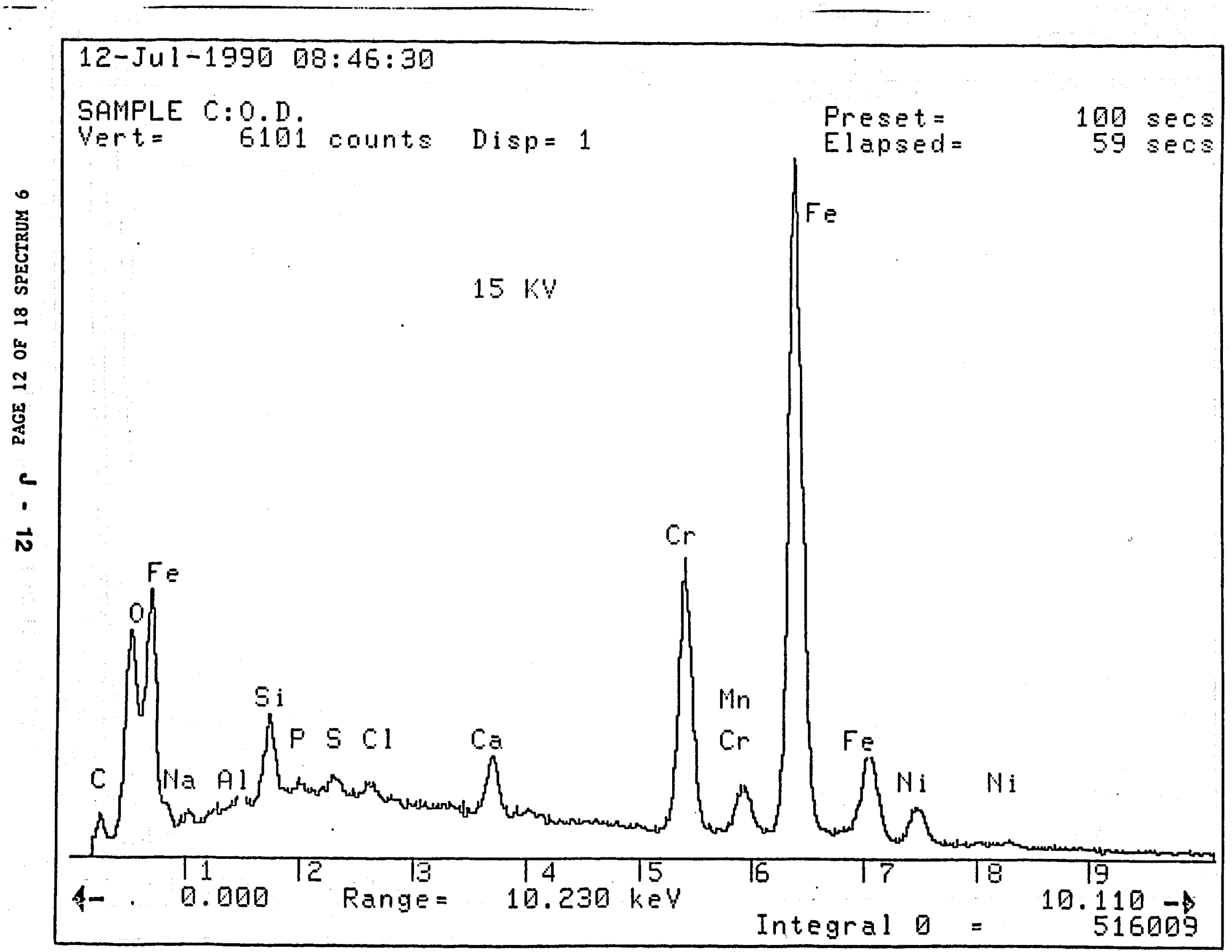




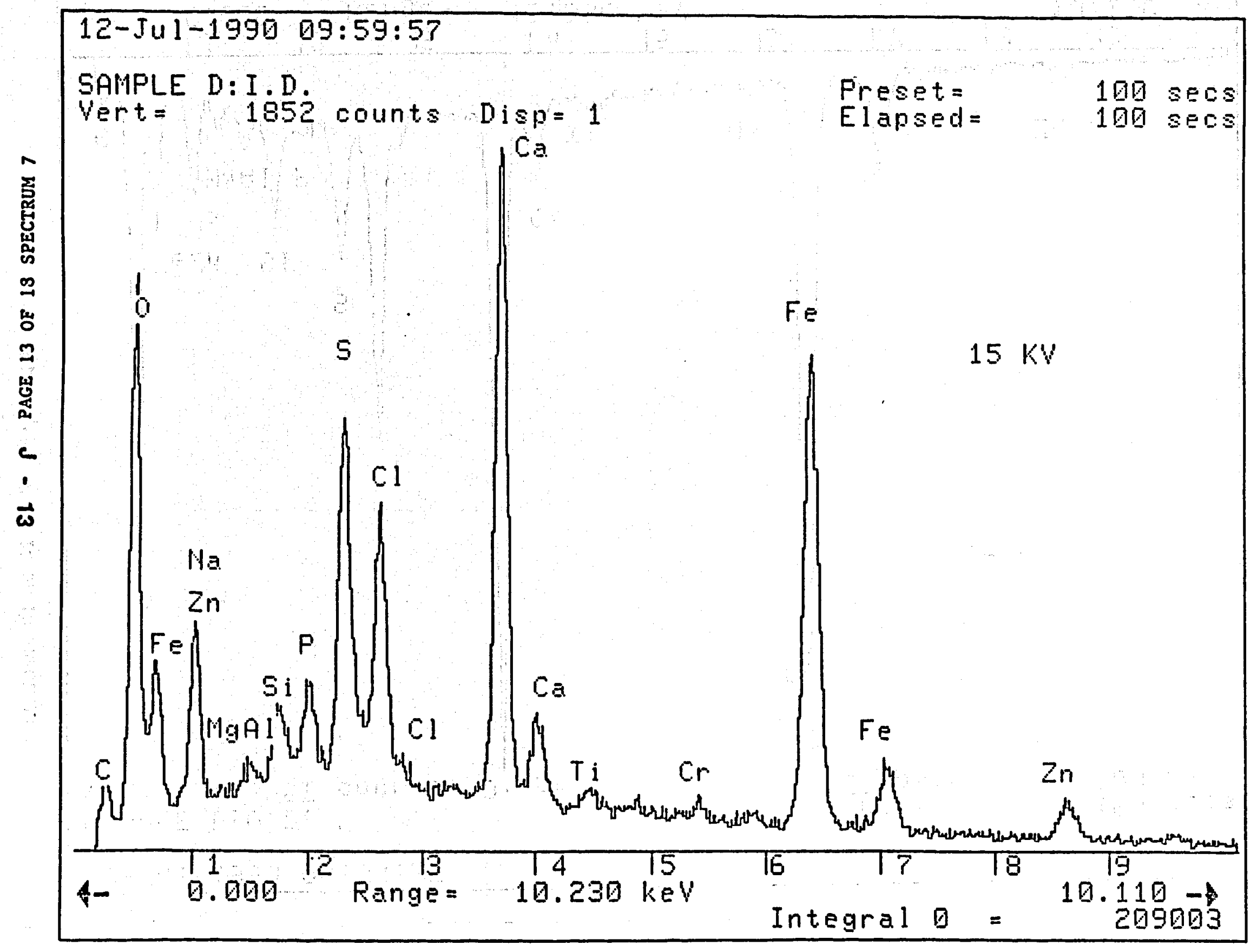




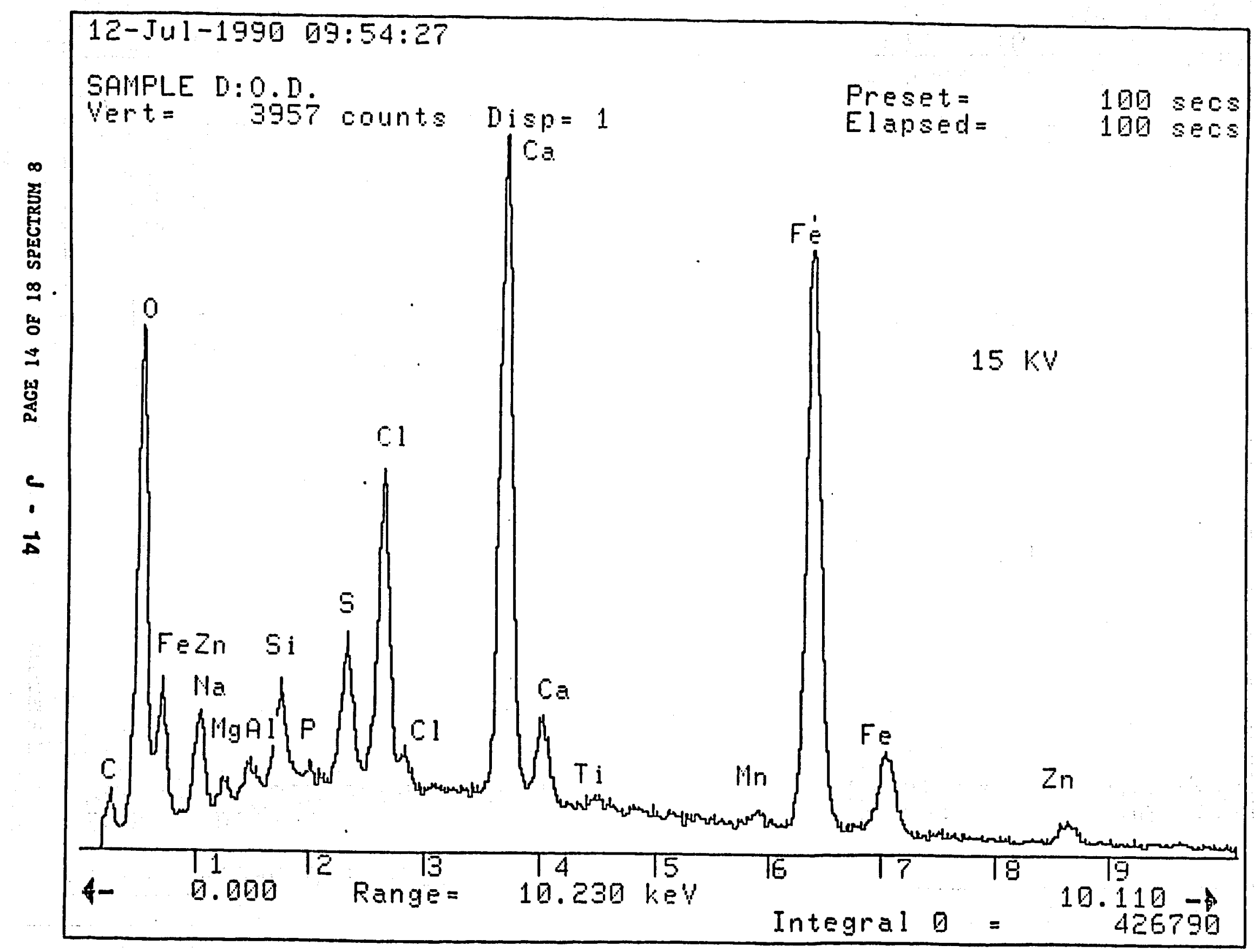




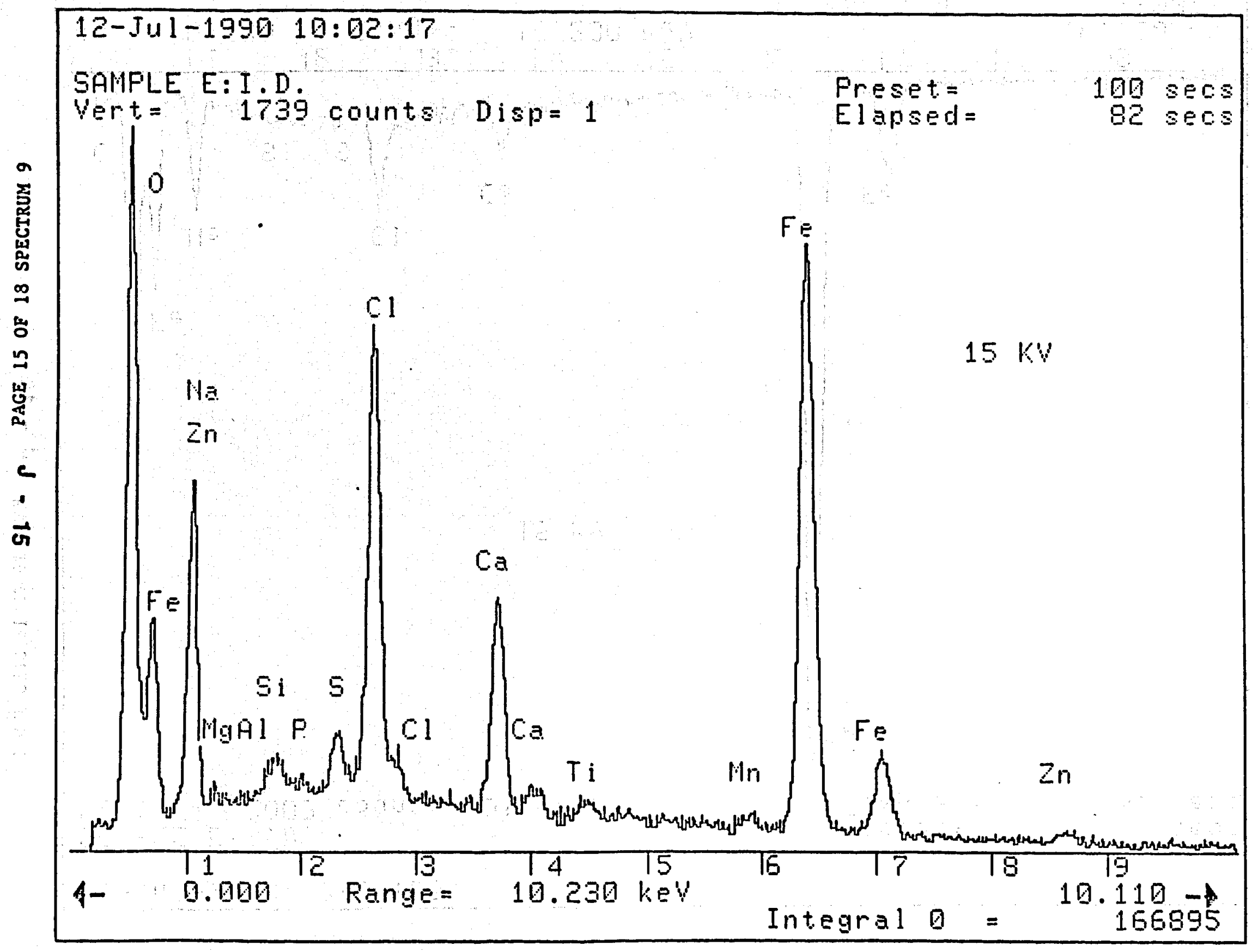




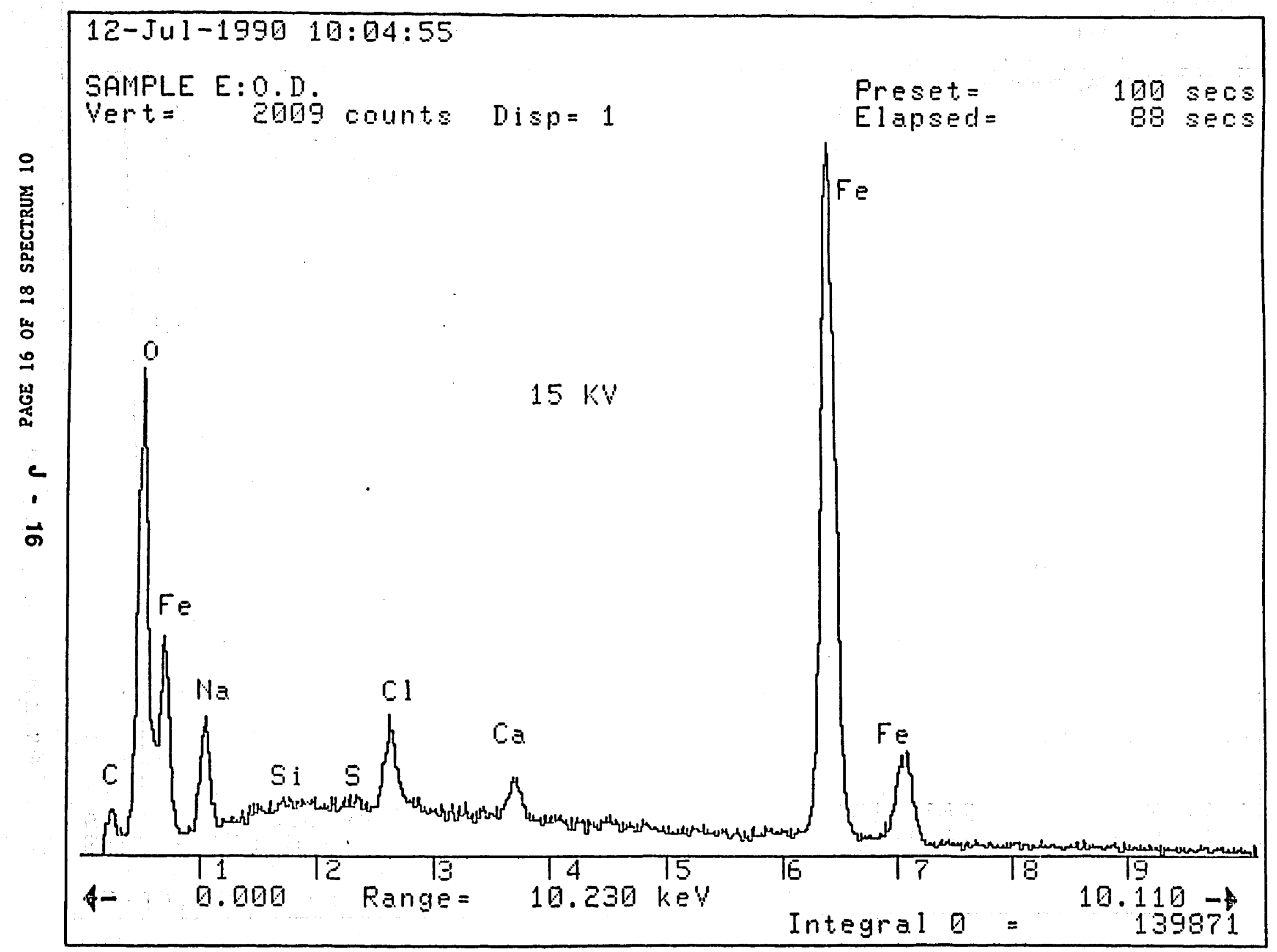




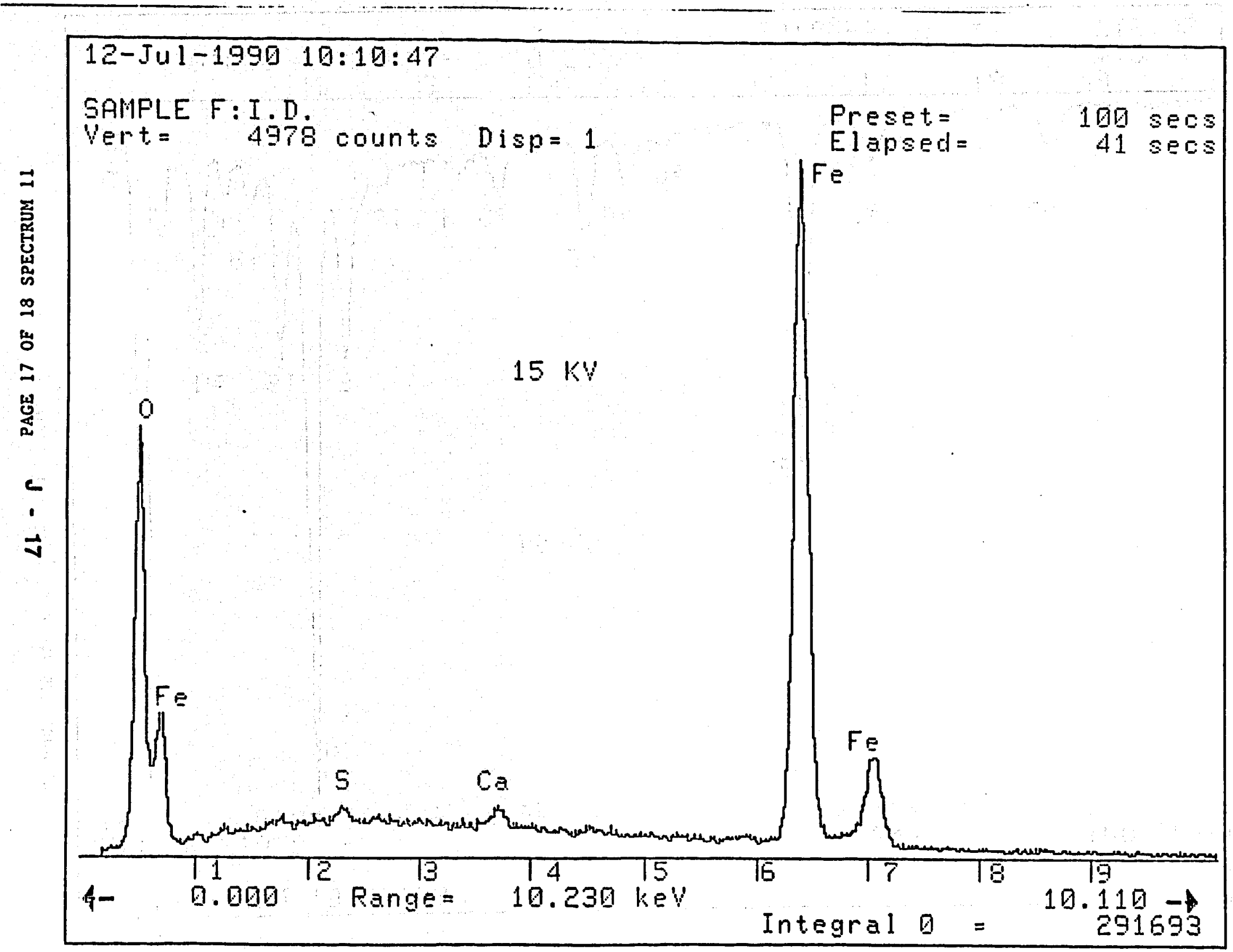




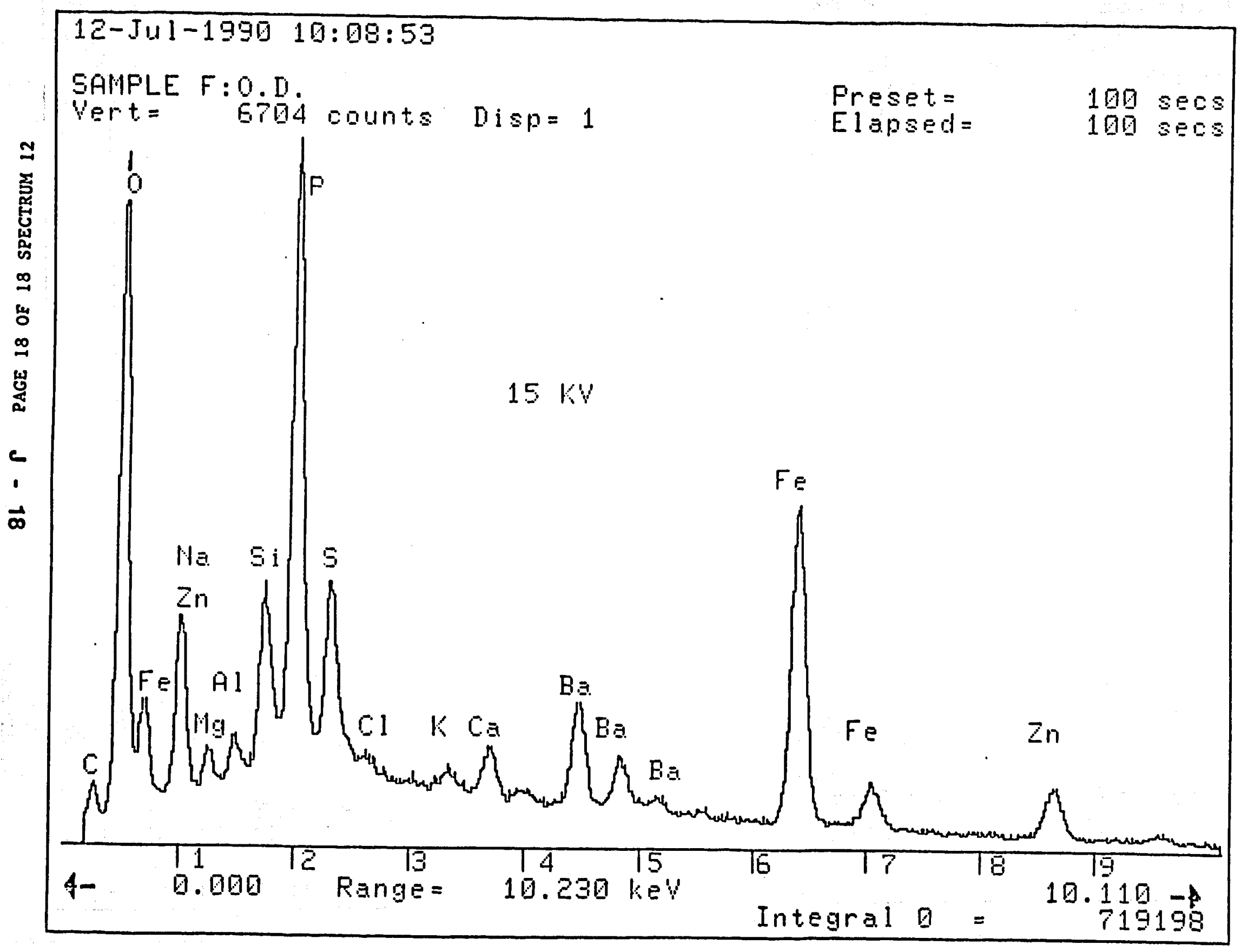




\section{CHEMICAL ANALYSIS}

\section{SAMPLES:}
A:
E-1-N TUBESIDE INLET
B:
E-1-N TUBESIDE OUTLET
C:
E-2-N SHELLSIDE
D:
E-3-N SHELLSIDE
E:
E-3-N TUBESIDE OUTLET
F:
E-3-N TUBESIDE INLET 


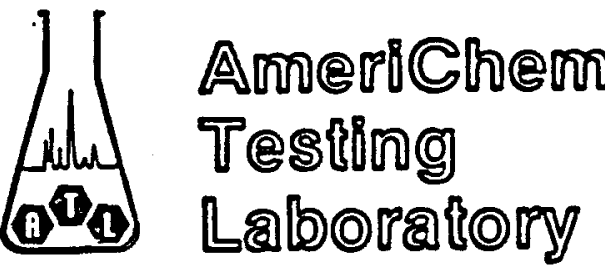 \\ REPORT NUMBER: AL-1879 CLIENT: \\ The Ben Holt Co. \\ 201 S. Lake Ave. \\ Pasadena CA 91101-3094}

\section{ANALYSIS}

Moisture

Qil

Identification of oil by IR Ash

Color of ash

Magnetics

Siliceous matter-soil, dirt

Chloride

Nitrate

Sulfate

Carbonate

Bicarbonate

Phosphate

Sodium

Calcium

Magnesium

Cadmium

Lead

Zine

Cobalt

Nickel

Manganese

Copper

Chromium

Iron, as iron oxide
1761 N. Batavis

Orange, CA 82665

(714) $821-1550$

FAX: (714) $821-4770$

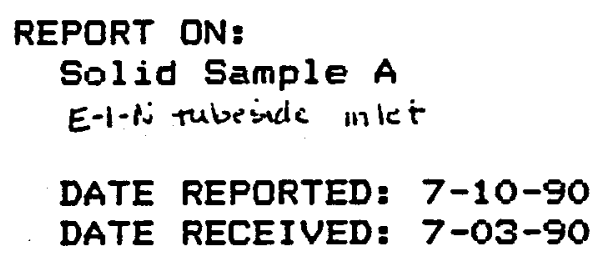

\section{REST RESULT}

$1.17 \%$
$51.37 \%$
Petroleum oil
$47.46 \%$
Light brown
Yes
$0.72 \%$
$0.20 \%$
<0.01\%
<0.01\%
<0.1\%
$0.005 \%$
$0.06 \%$
$0.281 \%$
$7.31 \%$
$0.237 \%$
$0.0169 \%$
$6.11 \%$
$4.18 \%$
N. D.
N. D.
$0.321 \%$
O. 205\%
N. D.
$4.63 \%$

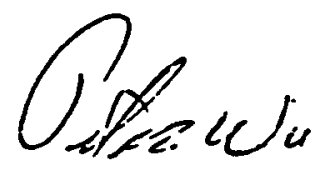

Peter T. Wis Lab Director 


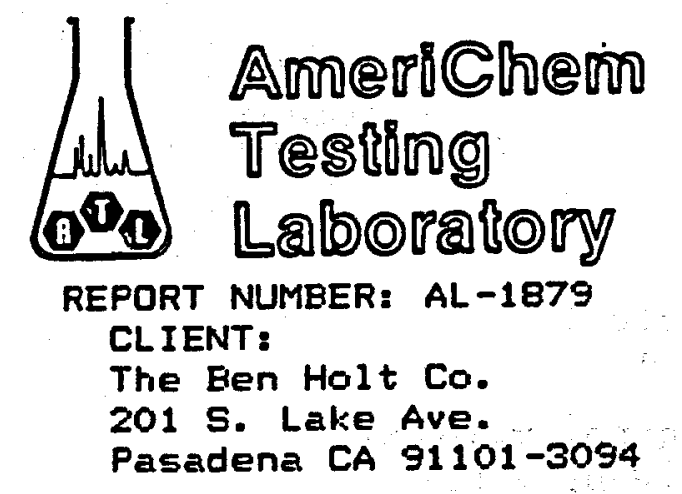

ANALYSIS

Moisture

Oil

Identification of oil by IR Ash

Color of ash

Magnetics

Siliceous matter-soil, dirt

Chloride

Nitrate

Sulfate

Carbonate

Bicarbonate

Phesphate

Sodium

Calcium

Magnesium

Cadmium

Lead

Zine

Cobalt

Nickel

Manganese

Copper

Chramium

Iron, as iron oxide
1781 N. Batavia

Orange, CA 02665

(714) $921-1550$

FAX: (714) 021-4770

\author{
REPORT ON: \\ Solid Sample B \\ i-H mercade curler \\ DATE REPORTED: 7-10-90 \\ DATE RECEIVED: 7-03-90
}

\section{REST RESULT}

\section{$2.51 \%$}

$21.5 E \%$

Petroleum oil

75. $52 \%$

Light yellow

slightly

2. $63 \%$

$0.16 \%$

$0.01 \%$

$<0.01 \%$

$0.41 \%$

$<0.005 \%$

$0.10 \%$

$0.259 \%$

$3.52 \%$

$0.155 \%$

$0.0380 \%$

10. $35 \%$

$11.94 \%$

N.D.

$0.0040 \%$

$0.171 \%$

$0.076 \%$

$0.040 \%$

$10.35 \%$

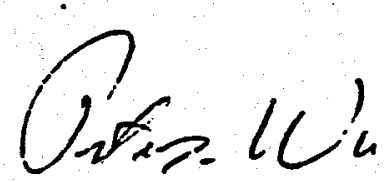

Peter T. Wu Lab Director 

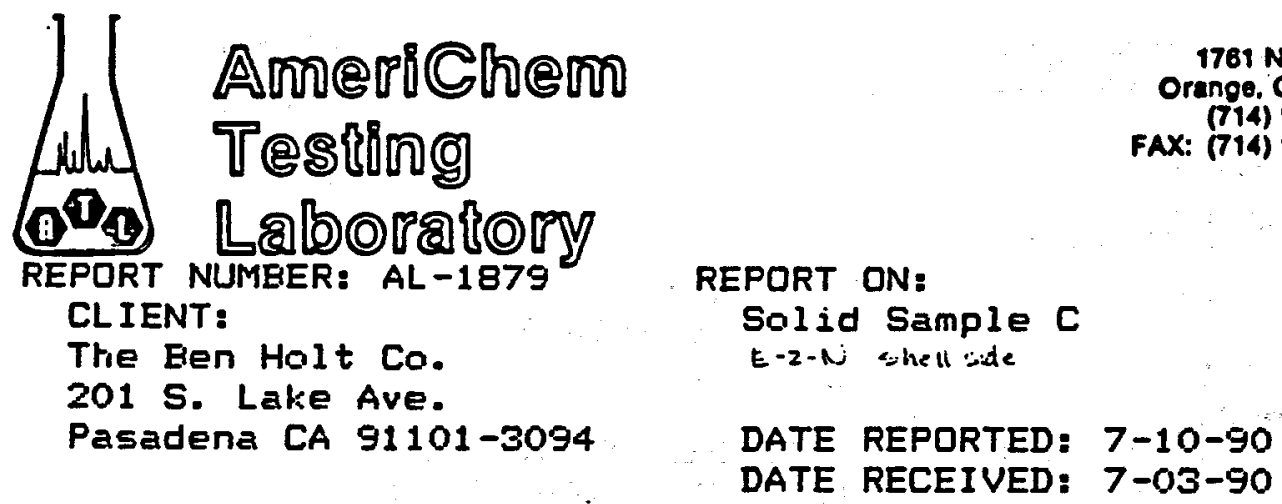

\section{ANALYSIS}

Moisture

Dil

Identification of ail by IR Ash

Color of ash

Magnetics

Silicegus matter-soil, dirt

Chloride

Nitrate

Sulfate

Carbonate

Bicarbonate

Phosphate

Sodium

Calcium

Magnesium

Cadmium

Lead

Zine

Cobalt

Nickel

Manganese

Copper

Chramium

Iron, as iron oxide

\section{REST RESULT}

$$
\begin{aligned}
& 3.40 \% \\
& 37.11 \% \\
& \text { Petraleum oil } \\
& 59.48 \% \\
& \text { Rust brown } \\
& \text { Stranly magnetic } \\
& 3.72 \% \\
& 0.81 \% \\
& 0.01 \% \\
& 0.01 \% \\
& 0.07 \% \\
& <0.005 \% \\
& <0.01 \% \\
& 0.183 \% \\
& 0.93 \% \\
& 0.105 \% \\
& N .0 . \\
& 0.0055 \% \\
& 0.278 \% \\
& 0.055 \% \\
& 0.0164 \% \\
& 0.149 \% \\
& 0.079 \% \\
& N .0 . \\
& 49.27 \%
\end{aligned}
$$

N.D. = Not detected

The main ingredient is iron rust and ail.

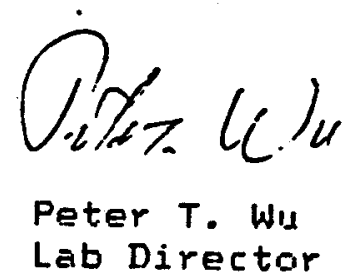




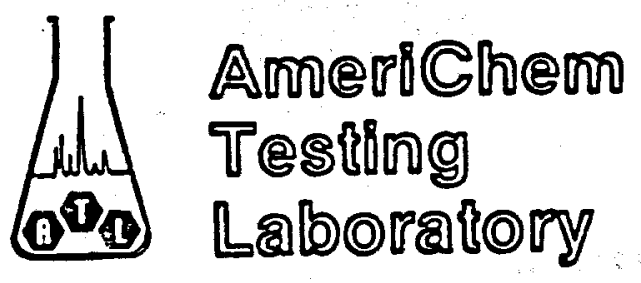

\section{REPORT NUMBER: AL-1879 \\ CLIENT: \\ The Ben Holt Co. \\ 201 5. Lake Ave. \\ Pasadena CA 91101-3094}

\section{ANALYSIS}

Moisture

Oil

Identification of oil by IR Ash

Color of ash

Magnetics

Siliceous matter-soil, dirt

Chloride

Nitrate

Sulfate

Carbonate

Bicarbonate

Phosphate

Sodium

Calcium

Magnesium

Cadmium

Lead

Zine

Cobalt

Nickel

Manganese

Copper

Chramium

Iron, as iran oxide
1781 N. Batevia

Orange. CA 92665

(714) $921-1550$

FAX: (714) $021-4770$

\section{REPORT QN: \\ Solid Sample D \\ : !.}

DATE REPORTED: $7-10-90$

REST RESULT

$1.11 \%$

$15.40 \%$

Petraleum oil

83. $49 \%$

Rust brown

Stronly magnetic

E. E3:

$0.02 \%$

$0.04 \%$

$<0.01 \%$

<. $0.1 \%$

$<0.005 \%$

$<0.01 \%$

$0.289 \%$

1. $37 \%$

$0.068 \%$

N. D.

$0.0105 \%$

$0.319 \%$

$0.088 \%$

$0.0248 \%$

$6.830 \%$

$0.093 \%$

$0.035 \%$

$75.06 \%$

N.D. = Not detected

The main ingredient is iron rust and oil.

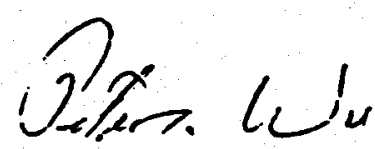

Peter T. Wu

Lab Director 


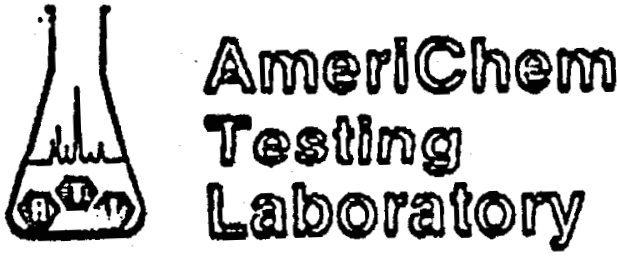 \\ REPORT NUMEER: AL-1875 CLIENT: \\ The Ben Holt Co. \\ 201 8. Lake Ave. \\ Pasadena CA 91101-3094}

\section{ANALYSIS}

Moisture

Chareonl particles

Oil

Identification of oil by IR Ast.

Color of ash

Magnetics

Sillceous matter-soil, dirt

Chlaride

Nitrate

Sulfate

Carbonate

Elcarbenata

Phosphate

Gidium

Calcium

Magnesium

Cadmliam

Lead

Zine

Cobalt

Nickel

Manganege

Copper

chromium

Iron, as Iran sixido
1701 N. Batavin

Oranco, Ca 02865

(7i4) $029-1880$

Fax: (7ib) ori-a7ro

\author{
REPORT ONI \\ Solid Sample E

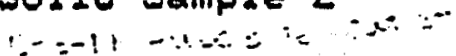 \\ DATE REFQRTED: $7-12-90$
DATE RECEIVED: $7-0,3-90$
}

\section{REET RESULT}

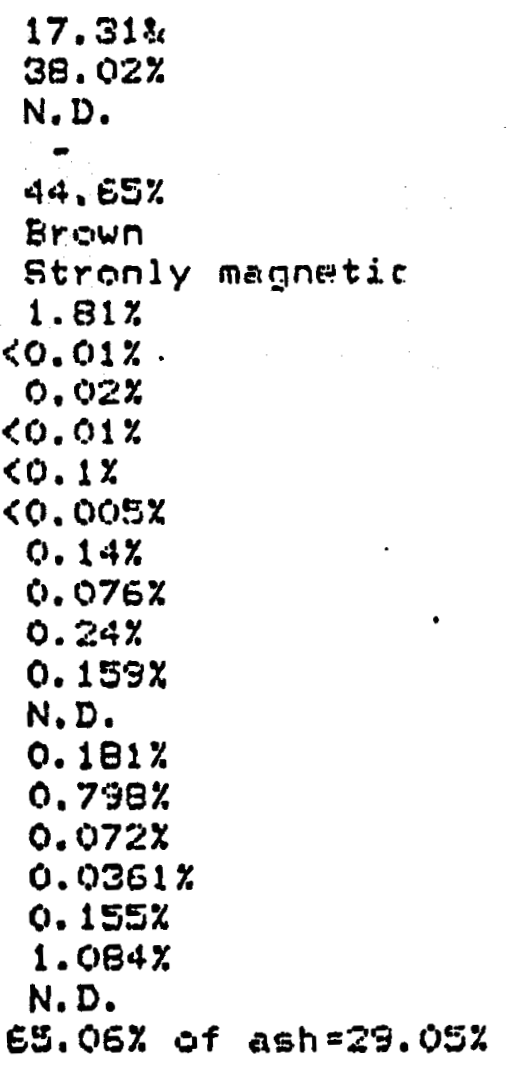

N.D. = Nat detected

The maln ingredient ds iron rust, charcoal particles and meisture.

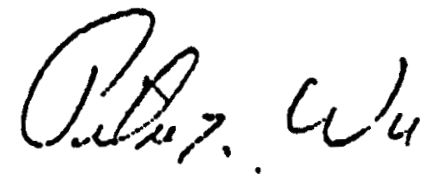

Peter T. Wh

Lab Directar 


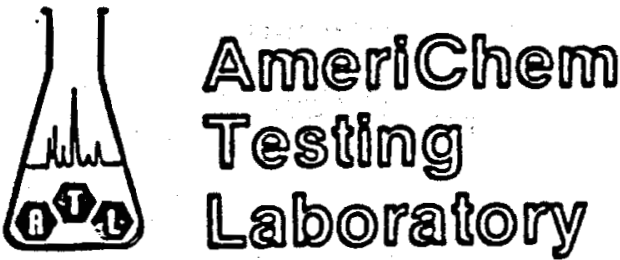
REPORT NUMBER: AL -1879 CLIENT:

The Ben Holt Co. 201 S. Lake Ave. Pasadena CA 91101-3094
1761 N. Batavla Orange. CA 92665

(714) $921-1550$

FAX: (714) $921-4770$

\section{ANALYSIS}

Moisture

Oil

Identification of oil by IR Ast.

Colar of ast.

Magnetics

Siliceous matter-soil, dirt

Chlaride

Fiber

Nitrate

Sulfate

Carbinate

Ei carbonate

Phosphate

Sigdium

Cal cium

Magnesium

Cadmium

Lead

Zine

Cobalt

Nickel

Manganese

Copper

Ctiromium

Iron, as iron oxide
REPORT QN:

Solid Sample F

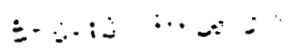

DATE REPORTED: 7-10-90

DATE RECEIVED: 7-03-90

\section{REST RESULT}

1. $68 \%$

N.D.

-

95. 53\%

Gray

Magnetic

$66.75 \%$

$<0.01 \%$

$0.3 \%$

$0.012 \%$

$<0.01 \%$

$0.11 \%$

$<0.005 \%$

$0.13 \%$

$0.224 \%$

$0.20 \%$

$0.075 \%$

N.D.

$0.007 \%$

$1.291 \%$

N.D.

$0.0238 \%$

$0.067 \%$

$0.094 \%$

N.D.

17.53\%

N.D. = Not detected

The main ingredient is iron rust and siliceous dirt.

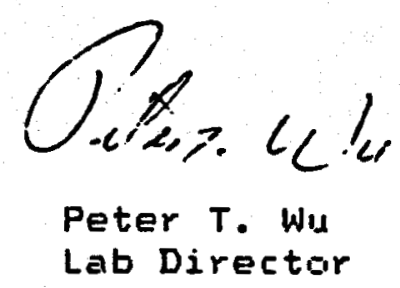




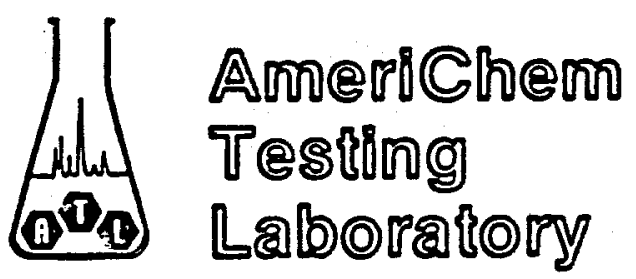

REPORT NUMBER: AL-1879

CLIENT:

The Ben Holt Co.

201 5. Lake Ave.

Pasadena CA 91101-3094

\section{ANALYSIS}

Maisture

Oil

Identification of oil by IR

Ash

Color of ash

Magnetics

Siliceous matter-soil, dirt

Chloride

Nitrate

Sulfate

Carbonate

Eicarbonate

Phosphate

Sodium

Calcium

Magnesium

Cadmium

Lead

Zine

Cobalt

Nickel

Manganese

Copper

Chramium

Iron, as iron oxide
1781 N. Batavie

Orange, CA 82665

(714) 921-1550

FAX: (714) $921-4770$

\section{REPQRT ON: \\ Solid Sample E-2-N, SHELL}

DATE REPORTED: $7-10-90$

DATE RECEIVED: 7-03-90

\section{REST : RESULT}

$41.54 \%$

$12.09 \%$

Petroleum ail

$46.37 \%$

Rust beown

Strangly magnetic

$9.92 \%$

$3.31 \%$

$<0.01 \%$

$<0.01 \%$

$1.60 \%$

$<0.005 \%$

$0.01 \%$

$0.697 \%$

$0.75 \%$

$0.045 \%$

N.D.

$0.006 \%$

$0.155 \%$

$0.047 \%$

$0.0134 \%$

$0.148 \%$

$0.049 \%$

N. D.

33. $48 \%$

N.D. = Not detected

The main ingredient is iron rust, oil and water.

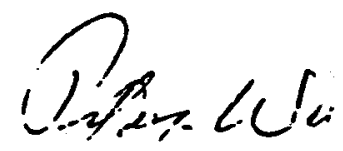

Peter T. Wu

Lab Director 


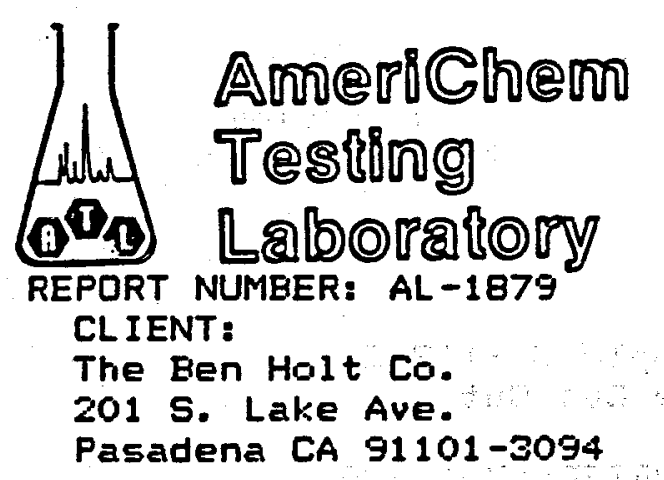

ANALYSIS

Moisture

Ash

Magnetic particles

Dirt particles

Sodium

Calcium

Magnesium

Cadmium

Lead

Zinc

Cobalt

Nickel

Manganese

Copper

Chromium

Iran

Viscosity, $100 \mathrm{~F}$

N.D. = Not detected
1761 N. Batavia

Oranoe, CA 02665

(714) $921-1550$

FAX: (714) 021-4770

\author{
REPORT ON: \\ Dil in $E-3-N$ shell \\ DATE REPORTED: $7-10-90$
DATE RECEIVED: $7-03-90$
}

REST RESULT

$<0.1 \%$

$0.017 \%$

$82.2 \mathrm{ppm}$

2.E Ppm

$0.0014 \%$

$0.0010 \%$

$0.00034 \%$

N.D.

$0.00033 \%$

$0.00233 \%$

N.D.

$0.0055 \%$

N.D.

N.D.

N.D.

$0.0086 \%$

The contamination came from rust and dirt particles.

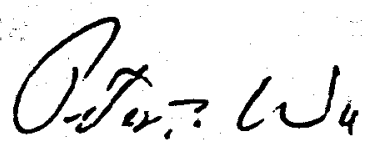

Peter T. Wis

Lab Director 


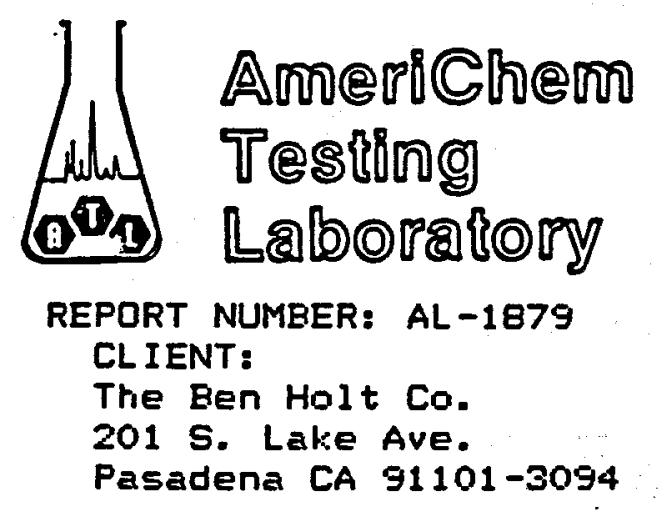

ANALYSIS

Moisture

Dil

Identification of ail by IR Ash

Color of ash

Magnetics

Siliceous matter-soil, dirt Chlaride

Nitrate

Sulfate

Carbonate

Bicarbonate

Phosphate

Sadium

Calcium

Magnesium

Cadmi um

Lead

Zine

Cobalt

Nickel

Manganese

Copper

Chromium

Iron, as iran oxide

N.D. = Nat detected

The main ingredient is iron rust and water.

$0.072 \%$
1761 N. Batavia Orange. CA 82665

(714) $021-1550$

FAX: (714) $821-4770$

\author{
REPORT ON: \\ Solid Sample CT-113-E \\ From Tube Cut Dut \\ DATE REPORTED: 7-10-90 \\ DATE RECEIVED: 7-03-90
}

\section{REST RESULT}

2E. $19 \%$

<. $1 \%$

$-$

$73.52 \%$

Rust beown

Strongly magnetic

$<0.1 \%$

$<0.1 \%$

$0.02 \%$

$<0.01 \%$

$1.60 \%$

$<0.1 \%$

$<0.01 \%$

$0.033 \%$

$0.04 \%$

$0.032 \%$

N.D.

$0.004 \%$

$0.093 \%$

$0.0066 \%$

$0.144 \%$

$0.015 \%$

$0.027 \%$

$68.21 \%$

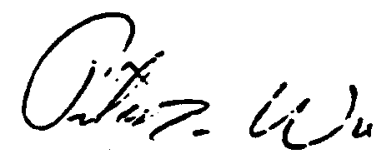

Peter T. Wu Lab Director 


\section{Americhem \\ Aul Testiong \\ (ADA LABOPROOYY}

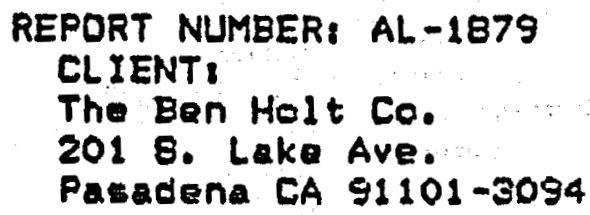

1761 N. Acturb Oranos, CA 02885 FAx: f7its 021-4780

\section{BAMPLE ID.}
A
$B$
C
D
F
$E-2-N$, SHELL
CT-113-E

$$
\begin{aligned}
& <0.5 \% \\
& <0.5 \% \\
& 1.5 \% \\
& 2.3 \% \\
& <0.5 \% \\
& 1.2 \%
\end{aligned}
$$$$
<0.5 \%
$$

\author{
REPORT ON: \\ Bolid Gample \\ DATE REPORTED: 7-12-90 \\ DATE RECEIVED: 7-03-90
}

CHARCQAL PARTICLES

For adjustment of calculation, please include the above \% in the oil content reported on July $10,1990$.

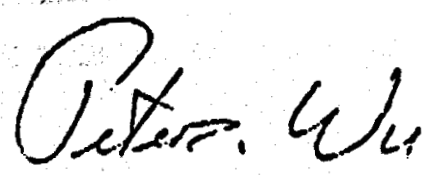

Pater T. Wu Lab Director 


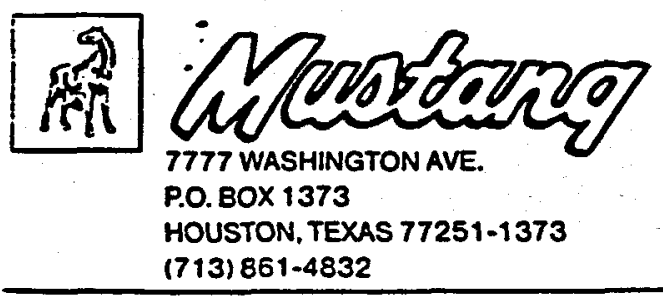

\section{RECEIVED \\ JUN 14990 \\ PROCLREMENT DIV.}

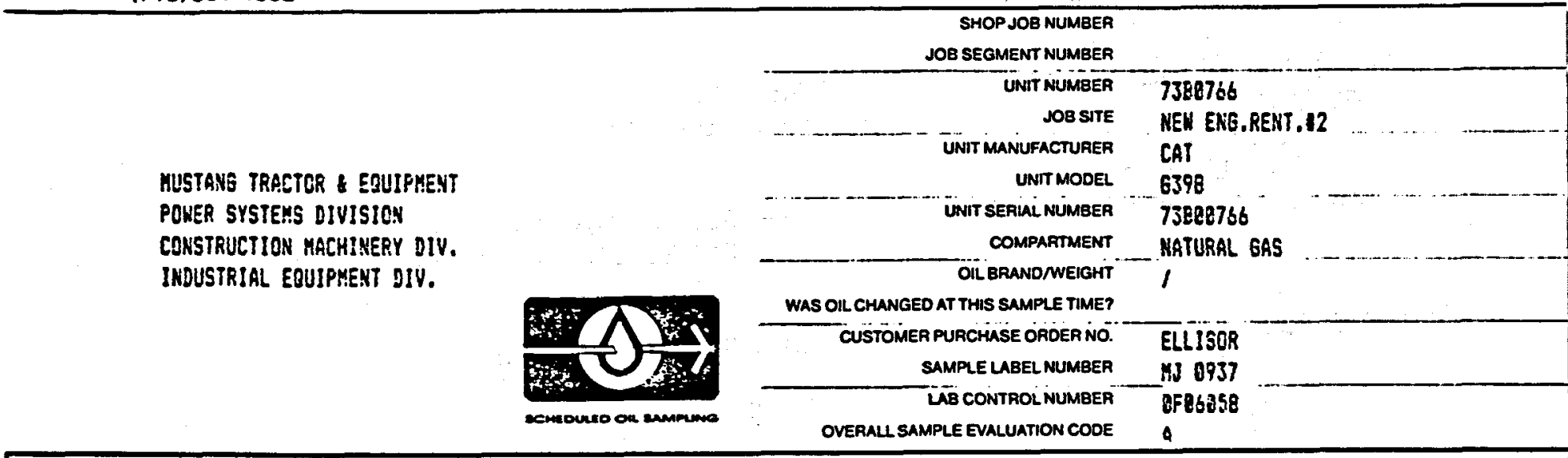

\begin{tabular}{|c|c|c|c|c|}
\hline $\begin{array}{l}\text { TAKEN } \\
253898 \\
\text { RECDO } \\
262198\end{array}$ & $\begin{array}{l}\text { Ann't. } \\
\text { of } \\
\text { Add } \\
\text { Oil }\end{array}$ & $\begin{array}{l}\text { SMU, SMU/ } \\
\text { Odom Odom. } \\
\text { on on } \\
\text { Unit Oil } \\
\\
3521 !\end{array}$ & Feedback: & MEAR KETAL RESULTS APPEAR NDRMAL. NO EVIDENEE OF C3NTAMINATION. \\
\hline
\end{tabular}

TAKEN JRON IS SLIEHTLY HIGK. RECBKKEKD OIL CHANGE RESAMPLE AFTER SBR HOURS

242498

RECD

$242599 \quad 34365 \quad 1115 \quad$ Feedback:

TAKEN UHXKOKN HSURS OK OIL SAYPLE. ELEKENTS APPEAR AVERAGE

238598

REC'D

$231298 \quad 33253 \quad$ Feedback:

TAKEN UWKNOHK ROURS OH OIL SAMPLE. ELEKENTS APPEAR AVERAGE

228698

RECD

228792

32613

Feedback:

\begin{tabular}{|c|c|c|c|c|c|c|c|c|c|c|c|c|c|c|c|c|c|}
\hline \multirow[t]{2}{*}{$\begin{array}{c}\text { Leo } \\
\text { Peoon } \\
\text { Date }\end{array}$} & \multicolumn{4}{|c|}{$\begin{array}{l}\text { Elements } \\
\text { Concentrations in PPM: }\end{array}$} & \multirow[b]{2}{*}{ AL } & \multirow[b]{2}{*}{ SI } & \multirow[b]{2}{*}{10} & \multirow[b]{2}{*}{$N A$} & \multicolumn{5}{|c|}{ Oil condition/Contaminants: } & & \multirow[b]{2}{*}{ VIS } \\
\hline & CU & FE & $C R$ & $P B$ & & & & & $y$ & $\mathbf{F}$ & A & ST & OX] & NIT & SUL & APP & \\
\hline $2 \leq 2198$ & 2 & 18 & 8 & 1 & $\theta$ & 1 & & 18 & NEG & & & $8 \%$ & & & & & \\
\hline 242598 & 2 & 28 & 1 & 3 & 1 & 3 & & 19 & NES & & & & & & & & \\
\hline 231298 & 2 & 16 & 1 & 3 & 1 & 1 & & 25 & KEE & & & & & & & & \\
\hline 220792 & 2 & 14 & 1 & 4 & 2 & 1 & & 22 & NEE & & & & & & & & \\
\hline
\end{tabular}

\section{ABBREVIATIONS}

\begin{tabular}{|c|c|c|c|c|c|}
\hline $\begin{array}{l}\text { Al - ALUMINUM } \\
\text { Cr - CHROMIUM } \\
\text { Fe - IRON }\end{array}$ & $\begin{array}{l}\text { Cu - COPPER } \\
\text { Pb - LEAD } \\
\text { MO- MOLYBDENUM }\end{array}$ & $\begin{array}{l}\text { Si - SILICON } \\
\mathrm{Na} \text { - SODIUM } \\
\text { TR - TRACE }\end{array}$ & $\begin{array}{l}\text { W-WATER } \\
\text { F-FUEL } \\
\text { A-ANTIFREEZE }\end{array}$ & $\begin{array}{l}\text { ST - SOOT } \\
\text { SUL-SULFUR } \\
\text { VIS - VISCOSITY }\end{array}$ & $\begin{array}{l}\text { OXI - OXIDATION } \\
\text { NIT - NITRATION } \\
\text { APP-APPEARANCE }\end{array}$ \\
\hline
\end{tabular}




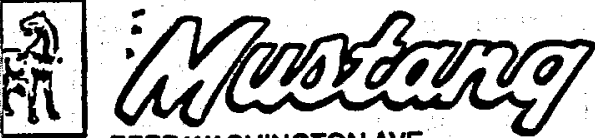 \\ 7777 WASHINGTONAVE. \\ P.O. BOX 1373 \\ HOUSTON, TEXAS 77251-1373 \\ (713) $861-4832$}

MUSTANG TRACTQR \& EOUIPMENT

POWER SYSTEKS DIVISION

CONSTRUCTION MACHINERY OIV.

INDUSTRIAL EQUIFMENT DIV.

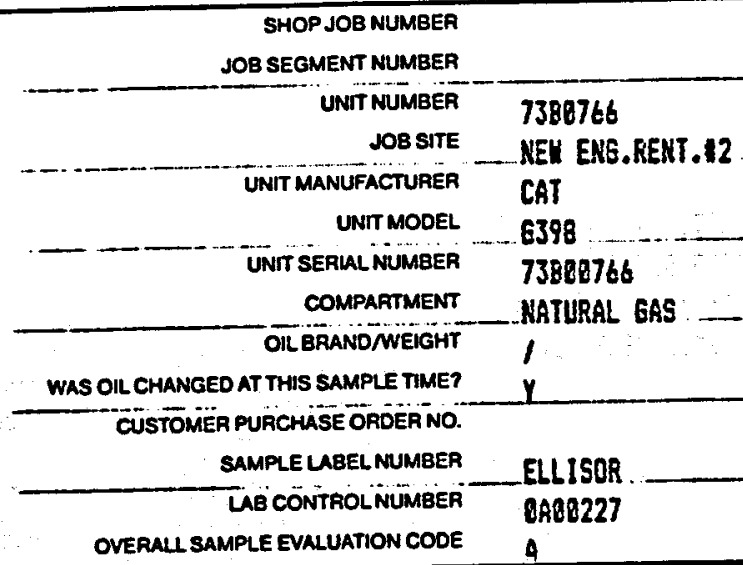

ALL ELEMENTS APPEAR ACCEPTABLE.

\begin{tabular}{|c|c|c|c|c|}
\hline $\begin{array}{l}\text { KEN } \\
898\end{array}$ & $\begin{array}{c}\text { Amit } \\
\text { of } \\
\text { Add } \\
\text { Oil }\end{array}$ & $\begin{array}{l}\text { SMUI } \\
\text { Odom } \\
\text { on } \\
\text { Unit }\end{array}$ & $\begin{array}{c}\text { SMU, } \\
\text { Odom. } \\
\text { on } \\
\text { Oil }\end{array}$ & $x=$ \\
\hline 198 & & 32812 & 1645 & edback: \\
\hline
\end{tabular}

KEN

$: 0$

Feedback:

KEN

C'D

Feedback:

KEN

CD

Feedback:

\section{Lab}

Report Elements

Date concentrations in PPM:

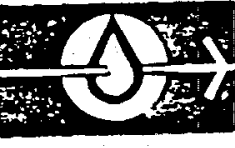

$$
198
$$

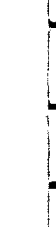

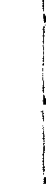

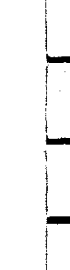




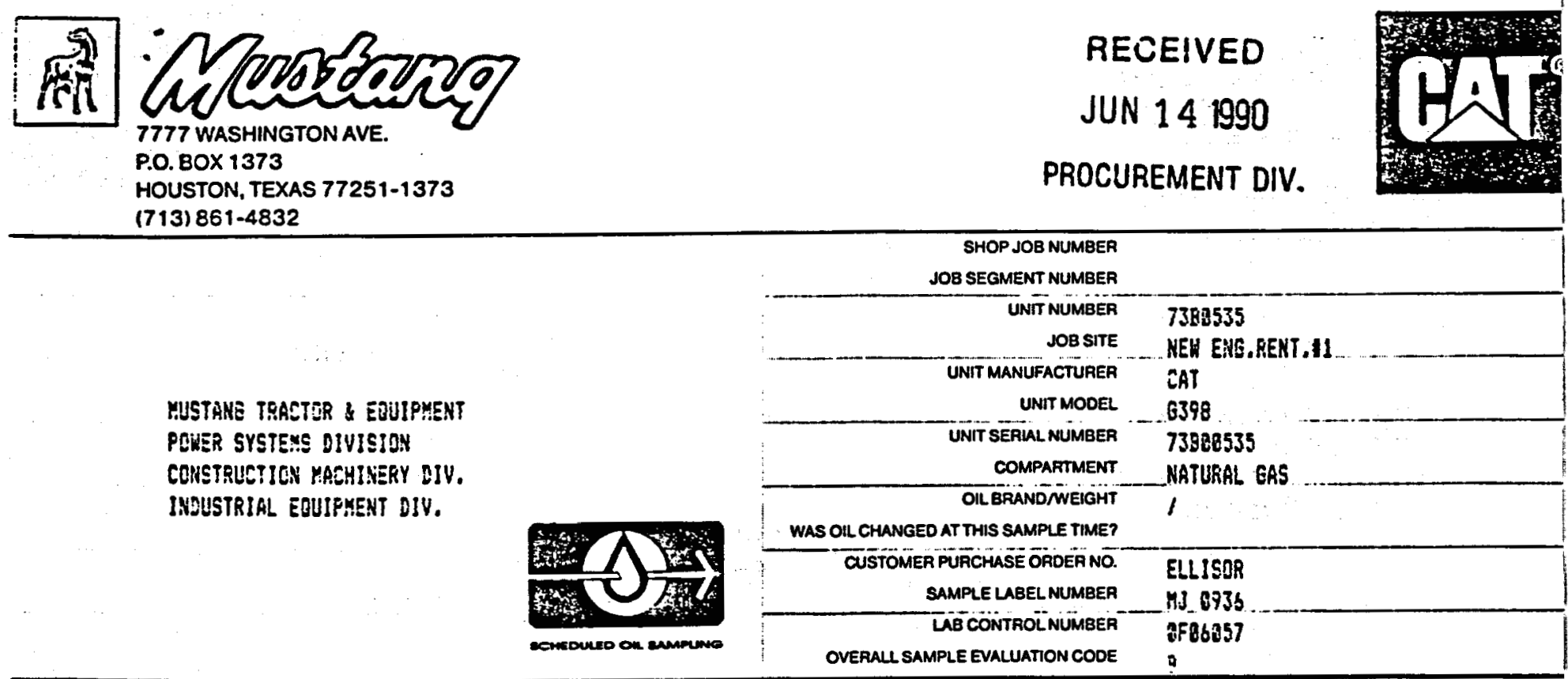

\begin{tabular}{|c|c|c|c|c|c|}
\hline $\begin{array}{l}\text { TAKEN } \\
\text { RESE90 } \\
\text { RECD } \\
\text { ISO198 }\end{array}$ & $\begin{array}{l}\text { Anil } \\
\text { of } \\
\text { Add } \\
\text { Oil }\end{array}$ & $\begin{array}{l}\text { SMUV } \\
\text { Odom. } \\
\text { on } \\
\text { Unit } \\
475 \\
\end{array}$ & $\begin{array}{l}\text { SMul } \\
\text { Odom. } \\
\text { on } \\
\text { Oil }\end{array}$ & Feedback: & $\begin{array}{l}\text { UNKNOWN HOURS OK OIL SAMPLE. IRON IS SLIGHTLY HIEH. } \\
\text { IT IS DIFFICUT TO INTERFRET RESULTS HITHOUT INFO. }\end{array}$ \\
\hline $\begin{array}{l}\text { TAKEN } \\
242499 \\
\text { REC'D } \\
242598 \\
\end{array}$ & & 3918 & 1132 & Feedback: & $\begin{array}{l}\text { COPPER IS SLIGHTLY HIGK. IRON IS HIGH. THIS KAY BE DUE TO AN EXTENDED OIL } \\
\text { CHANGE PERIOD. } \\
\text { RECOMMEND OIL CYANGE RESAMPLE AFTER } 528 \text { HOURS }\end{array}$ \\
\hline $\begin{array}{l}\text { TAKEN } \\
232598 \\
\text { RECD } \\
231298\end{array}$ & & 2779 & & Feedback: & $\begin{array}{l}\text { UKKNOUY HOURS ON OIL SAKPLE. SILICAK IS SLIGHTLY HIEH. MODERATE DIRT ENTAY } \\
\text { EVIDENT } \\
\text { THIS SAYPLE CONTAINS A SLIGHT HATER CONTAKIHATIOK. RECOMKEND OIL CHANGE }\end{array}$ \\
\hline $\begin{array}{l}\text { TAKEN } \\
222898 \\
\text { RECD } \\
322798 \\
\end{array}$ & & 2149 & & Feedback: & UHKNOHN HOURS ON OIL SAMPLE. ELEKENTS APPEAR AVERAGE \\
\hline
\end{tabular}

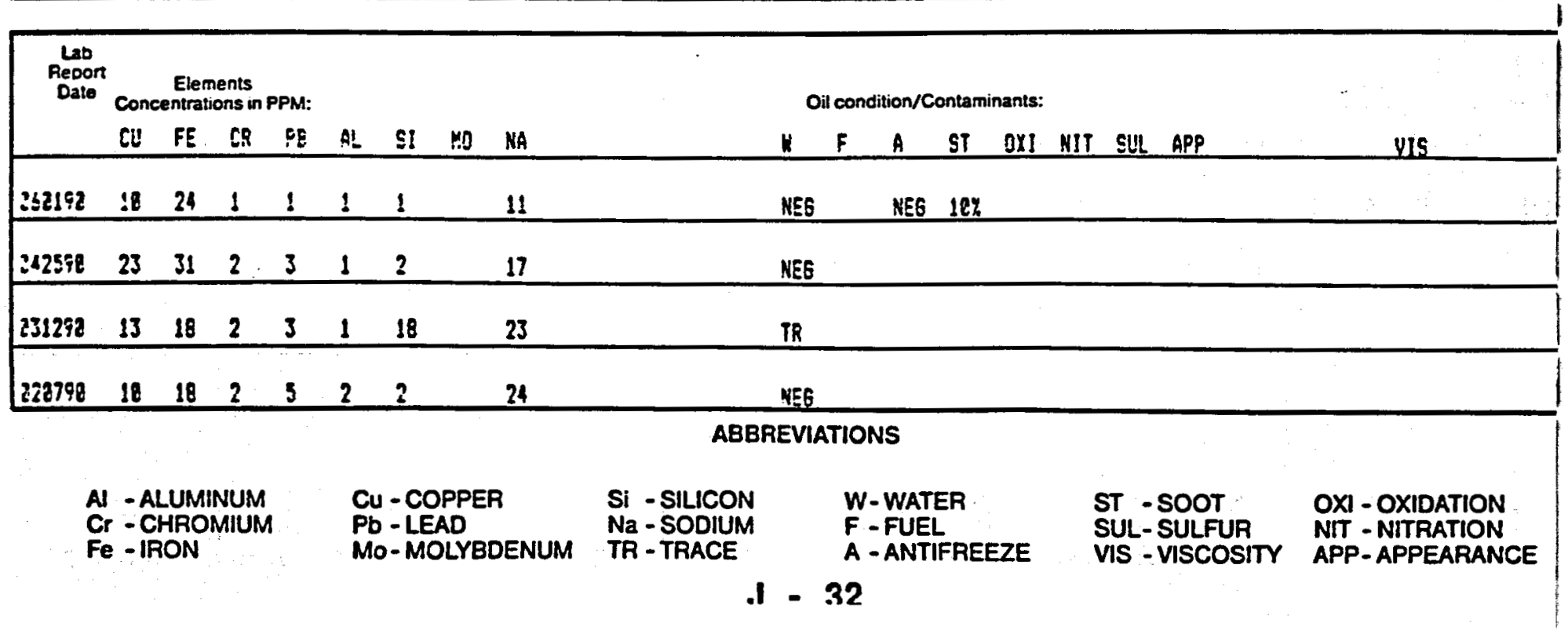


MUSTANG TRACTOR \& EOUIPKENT

POUER SYSTEMS OIUISIOK

CONSTRUCTION MACHINERY DIV.

INDUSTRIAL EQUIPKENT DIV.

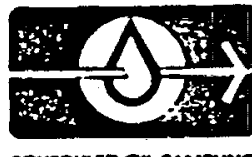

cencoures on sammine
SHOP JOB NUMBER
JOB SEGMENT NUMBER

UNIT MUMBER JOB SITE UNIT MANUFACTURER UAMT MODEL UNIT SERIAL NUMBER COMPAFTMENT OIL BRANOMNEIGHT
WAS OLL CHANGED AT THIS SAMPLE TIME? CUSTOMER PURCHASE OADER NO. SAMPLE LABEL NUMBER LAB CONTAÓL NUMBER OVERALL SAMPLE EVALUATION CODE

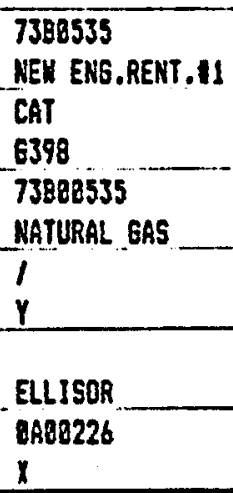

KEN Am't SMU/ SMU/
of Odom. Odom
1898 Add on on
Oil Unit Oil

C'D

198 $1553 \quad 1553$
COFPER IS HIGH. IRON IS HIEH. ALUHINUK IS EXTREMELY HIGH.

POSSIBLE RODIMAIN BEARINE MEAR. RESAKPLE IMKEDIATELY TO CHECK.

KEN

C'D

Feedback:

KEN

co

Feedback:

KEN

c'o

Feedback:

Date Concentrations in PPM:

Oil condition/Contaminants:

CU FE CR PB AL SI HO NA

N $F$ A ST OXI NIT SUL APP

VIS

$\begin{array}{lllllll}198 & 38 & 38 & 1 & 12 & 13 & 2\end{array}$

23

NES

22\% 42\% 75\% $57 \%$

\section{ABBREVIATIONS}

$\begin{array}{lllll}\text { Al - ALUMINUM } & \text { CU-COPPER } & \text { Si - SILICON } & \text { W-WATER } & \text { ST - SOOT } \\ \text { Cr - CHROMIUM } & \text { Pb-LEAD } & \text { Na - SODIUM } & \text { F-FUEL } & \text { SUL-SULFUR } \\ \text { Fe - IRON } & \text { MO-MOLYBDENUM - NIDATION } & \text { TR - TRACE } & \text { A-ANTIFREEZE } & \text { VIS - VISCOSITY APP-APPEARANCE }\end{array}$




\section{APPENDIX $\mathrm{K}$}

\section{MANAGEMENT REPORT DATA SHEETS}

JANUARY 5, 1990 - MAY 29, 1990

THE BEN HOLT CO. 
January 5-8, 1990

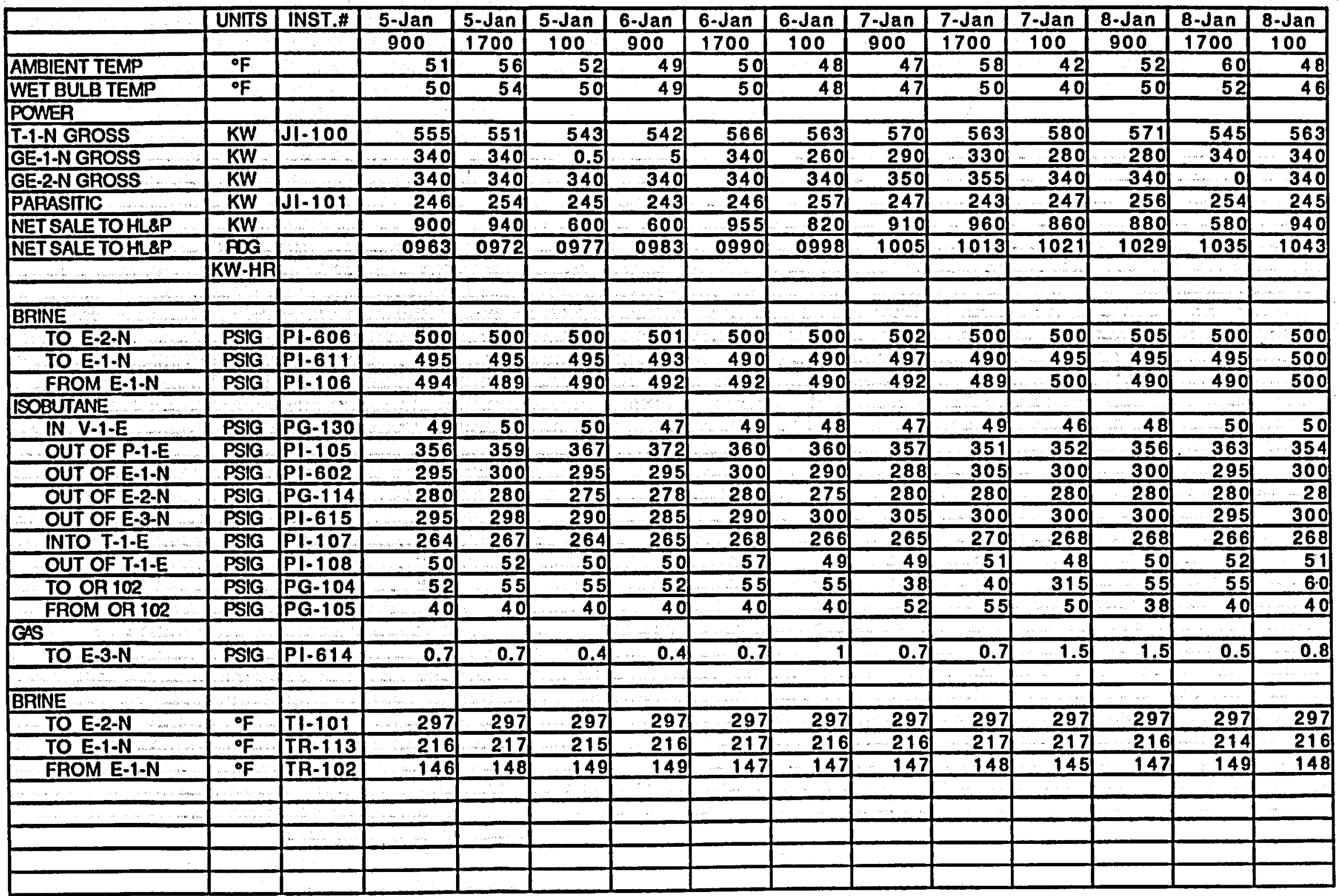




\section{GEOPRESSURED HYBRID POWER SYSTEM - MANAGEMENT REPORT DATA}

January $5.8,1990$

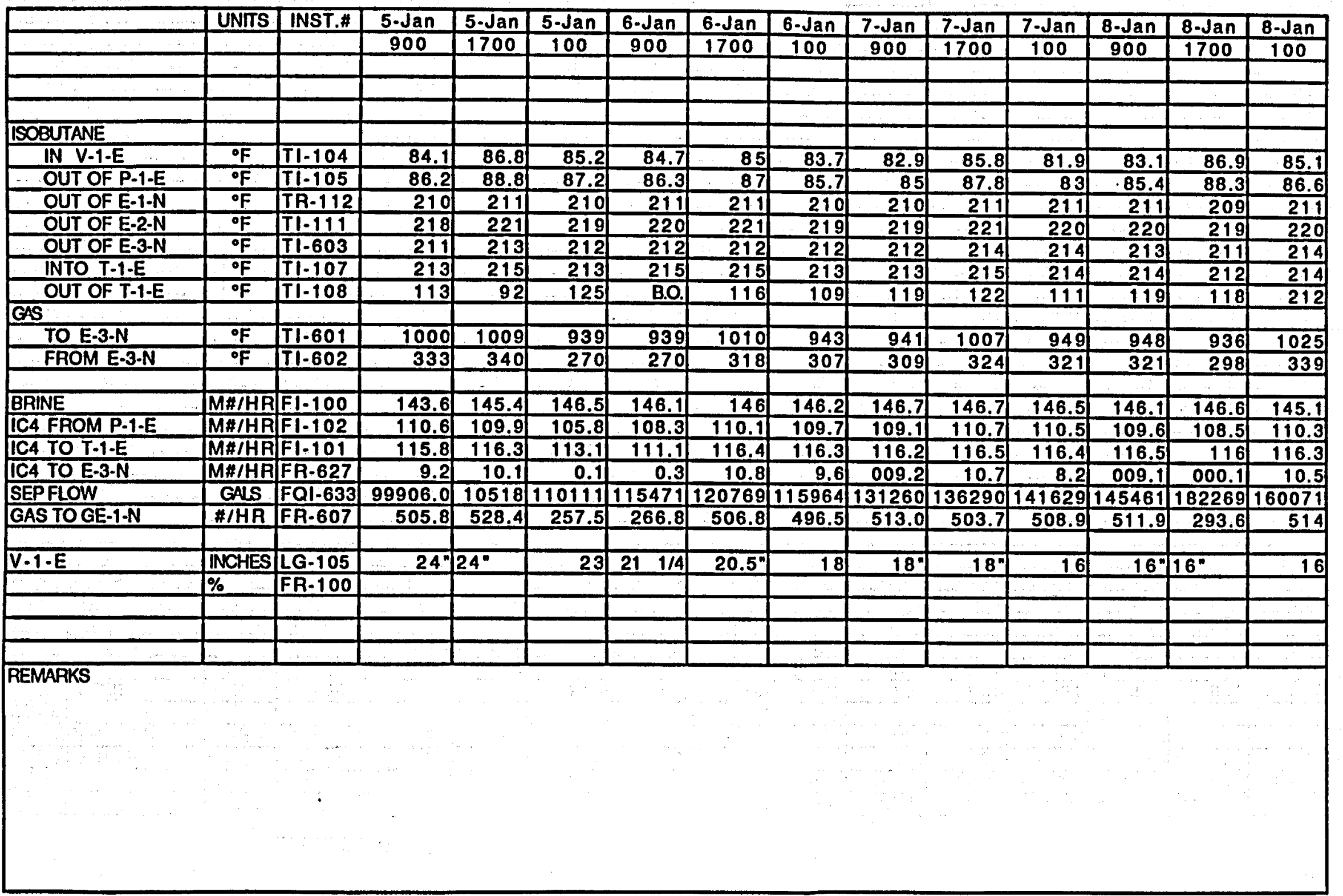


January 9.10, 1990

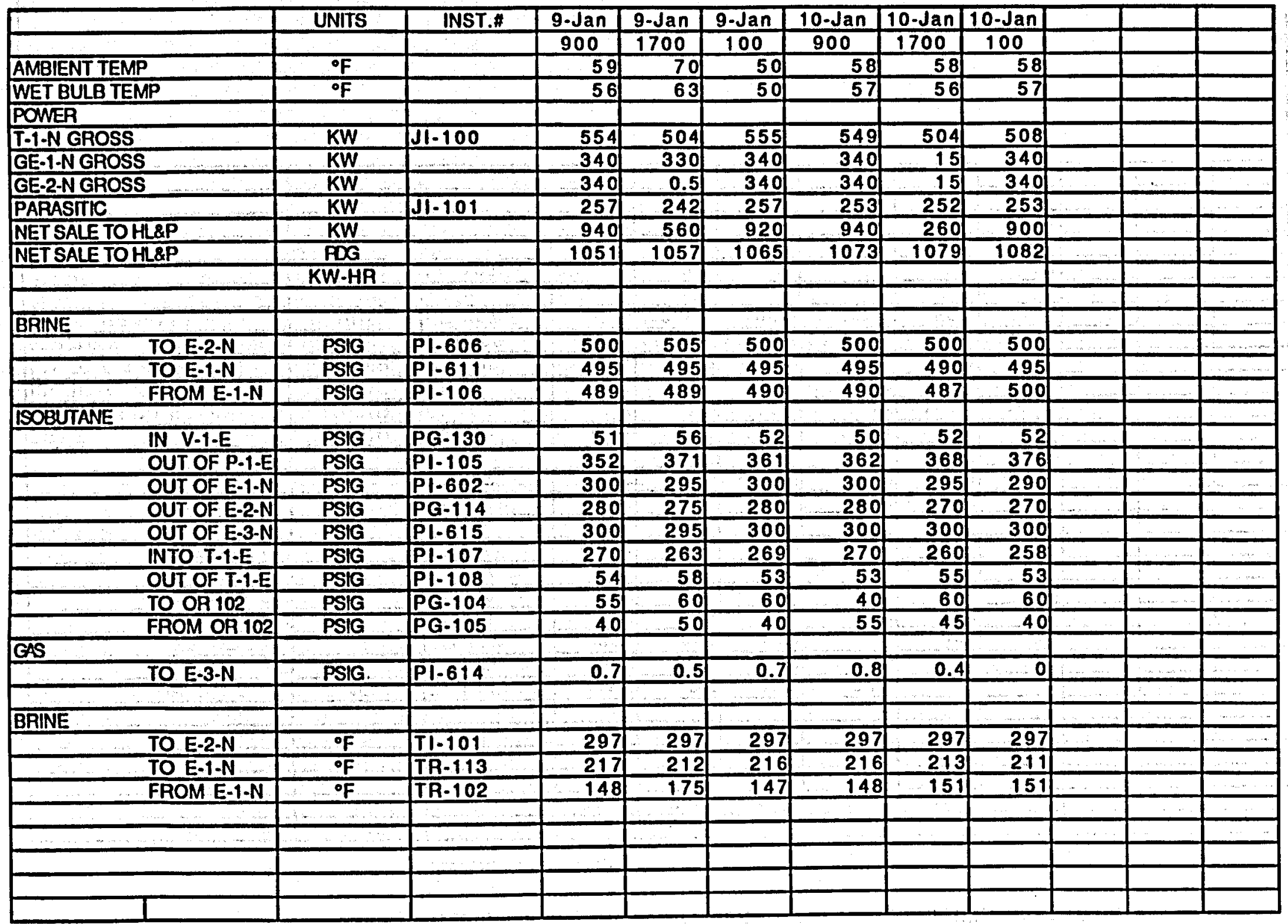


GEOPRESSURED HYBRID POWER SYSTEM - MANAGEMENT REPORT DATA

January 9-10, 1990

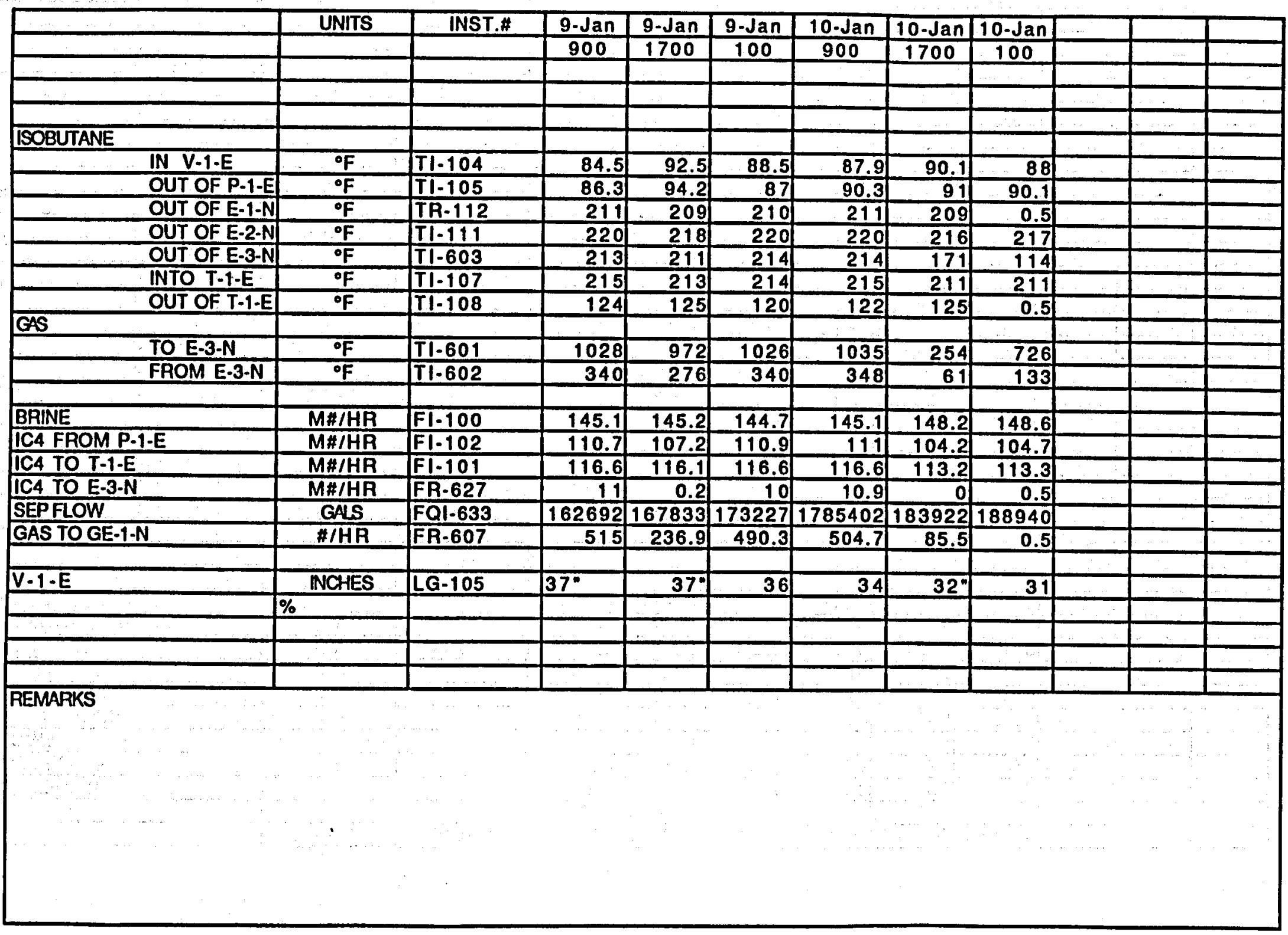


January 11-14, 1990

\begin{tabular}{|c|c|c|c|c|c|c|c|c|c|c|c|c|c|c|}
\hline & UNTS & INST.\# & 11-Jan & 11-Jan & 11-Jan & 12-Jan & 12-Jan & 12-Jan & 13-Jan & 13-Jan & 13-Jan & 14-Jan & 14-Jan & 14-Jan \\
\hline & & & 900 & 1700 & 100 & 900 & 60 & 100 & 900 & 1700 & 100 & 900 & 1700 & 100 \\
\hline AMBIENT TEMP & ${ }^{\circ} \mathrm{F}$ & & 64 & 66 & 60 & 55 & 0.5 & 42 & 48 & 51 & 52 & 63 & 65 & 62 \\
\hline WET BULB TEMP & of & & 63 & 62 & 59 & 53 & 50 & 36 & 43 & 46 & 50 & 60 & 62 & 60 \\
\hline POWER & & & & & & & & & & & & 537 & & \\
\hline T-1-N GROSS & $\mathrm{KW}$ & $J 1-100$ & 528 & 526 & 532 & 562 & 566 & 583 & 574 & 558 & 555 & 340 & 521 & 528 \\
\hline GE-1-N GROSS & $\mathrm{KW}$ & & 240 & 330 & 340 & 340 & 320 & 340 & 340 & 340 & 340 & 340 & 340 & 340 \\
\hline GE-2-N GAOSS & $\mathrm{KW}$ & & 340 & 330 & 340 & 340 & 340 & 340 & 340 & 340 & 340 & 243 & 340 & 340 \\
\hline PARASITIC & $\mathrm{KW}$ & $J 1-101$ & 253 & 251 & 254 & 254 & 244 & 256 & 246 & 254 & 243 & 900 & 252 & 253 \\
\hline NET SALE TO HL\&P & KW & & 820 & 880 & 920 & 940 & $900-940$ & 940 & 940 & 906 & 930 & 1160 & 910 & 920 \\
\hline NET SALE TO HL\&P & $\mathbf{P D G}$ & & 1089 & 1095 & 1103 & 1111 & 1119 & 1126 & 1135 & 1143 & 1151 & 1029 & 1168 & 1175 \\
\hline & KW.HA & & -1. & & & & & & & & $\ldots \ldots$ & & & $\ldots$ \\
\hline & & & & & & & & & & & & & & \\
\hline BAINE & & & , & & & & & & & & & & & \\
\hline TO E-2-N & PSIG & $\mathrm{P} \mid .606$ & 500 & 510 & 500 & 501 & 500 & 500 & 500 & 500 & 500 & 500 & 500 & 500 \\
\hline TO E.1-N & PSIG & P1.611 & 498 & 495 & 495 & 495 & 495 & 490 & 495 & 490 & 490 & 490 & 490 & 490 \\
\hline FROM E-1-N & PSIG & $P \mid-106$ & 494 & 491 & 490 & 491 & 487 & 490 & 490 & 490 & 490 & 487 & 485 & 490 \\
\hline ISOBUTANE & 5 & & & & & & & & & & & & & \\
\hline IN V-1-E & PSIG & PG-130 & 54 & 57 & 56 & 46 & 49 & 44 & 44 & 49 & 50 & 54 & 56 & 56 \\
\hline OUT OF P-1-E & PSIG & P/.105 & 369 & 347 & 358 & 359 & 348 & 346 & 352 & 361 & 358 & 363 & 367 & 360 \\
\hline OUT OF E-1-N & PSIG & PI.602 & 300 & 300 & 295 & 295 & 300 & 300 & 300 & 300 & 295 & 300 & 300 & 300 \\
\hline OUT OF E-2-N & PSIG & PG-114 & 280 & 280 & 275 & 280 & 280 & 280 & 280 & 280 & 280 & 280 & 280 & 280 \\
\hline OUT OF E-3-N & PSIG & PI.615 & 295 & 300 & 300 & 300 & 300 & 300 & 300 & 300 & 300 & 300 & 300 & 300 \\
\hline INTO T.1.E & PSIG & $P \mid-107$ & 267 & 270 & 268 & 262 & 268 & 266 & 266 & 266 & 269 & 269 & 268 & 269 \\
\hline OUT OF T-1-E & PSIG & $P \mid-108$ & 58 & 59 & 56 & 49 & 51 & 46 & 50 & 50 & 52 & 56 & 58 & -58 \\
\hline TO OR 102 & PSIG & PG-104 & 60 & 62 & 60 & 50 & 50 & 50 & 50 & 50 & 30 & 60 & 60 & 60 \\
\hline FROM OA 102 & PSIG & PG.105 & 50 & 50 & 45 & 35 & 40 & 35 & 35 & 40 & 55 & 45 & 50 & 45 \\
\hline GAS & & & & & & & & & & & & & & \\
\hline TO E-3-N & PSIG & $P \mid-614$ & 0.7 & 0.7 & 0.8 & 0.5 & 0.7 & $.5-1.0$ & 0.1 & 0.8 & $.5-1.0$ & 0.8 & 0.8 & 0.8 \\
\hline & & & & & & & & & $\therefore$ & & $\ldots \ldots$ & & $\cdots$ & $\cdots \cdots$ \\
\hline BRINE & & & & & & & & & & & & & & $\ldots$ \\
\hline TO E-2-N & ${ }^{\circ} \mathrm{F}$ & $T 1.101$ & 297 & 297 & 297 & 296 & 297 & 297 & 296 & 296 & 296 & 297 & 297 & 297 \\
\hline TO E-1-N & ${ }^{\circ} \mathrm{F}$ & TR-113 & 0.5 & 217 & 0.5 & 216 & 0.3 & 0.5 & 216 & 216 & 216 & 15 & 216 & 215 \\
\hline FAOM E-1-N & OF & TR-102 & 0.5 & 147 & 0.5 & 147 & 0.5 & 0.5 & 146 & 148 & 148 & 15 & 151 & 15.0 \\
\hline & & & & & & & & & & & & $\cdots$ & & \\
\hline & & & & & & & & & & & & & & \\
\hline & & & & & & & & & & & & & & \\
\hline & & & & & & & & & & & & & & \\
\hline & & & & & & & & & & & & & & \\
\hline
\end{tabular}


GEOPRESSURED HYBRID POWER SYSTEM - MANAGEMENT REPORT DATA

January 11-14, 1990

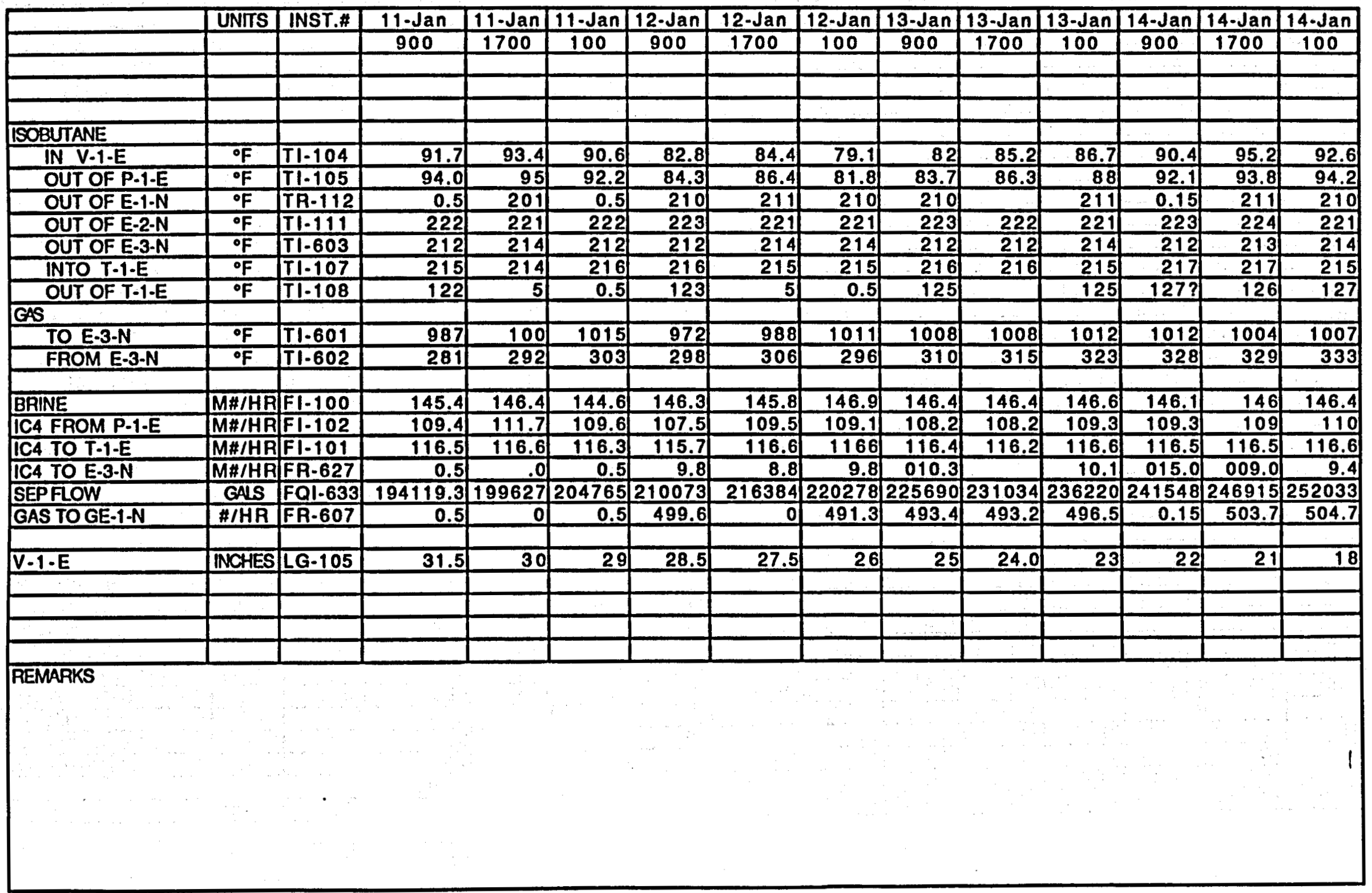


January $15.17,1990$

\begin{tabular}{|c|c|c|c|c|c|c|c|c|c|c|}
\hline & INST.\# & $15 \cdot \operatorname{Jan}$ & 15-Jan & $15 \cdot \mathrm{Jan}$ & 16-Jan & 16-Jan & 16-Jan & 17-Jan & 17-Jan & 17-Jan \\
\hline & & 900 & 1700 & 100 & 900 & 1700 & 100 & 900 & 1700 & 100 \\
\hline AMBIENT TEMP & & 65 & 67 & 60 & 67 & 68 & 64 & 69 & 70 & 67 \\
\hline WET BULB TEMP & & 62 & 63 & 60 & 65 & 66 & 64 & 67 & 68 & 67 \\
\hline \multicolumn{11}{|l|}{ POWEA } \\
\hline T-1-N GAOSS & $J 1-100$ & 517 & 519 & 525 & 515 & 505 & 514 & o & 500 & 514 \\
\hline GE-1-N GROSS & & 340 & 340 & 340 & 340 & 340 & 340 & 340 & 340 & 340 \\
\hline GE-2-N GROSS & & 340 & 340 & 340 & 340 & 340 & 340 & 340 & 340 & 340 \\
\hline PARASTTIC & J1.101 & 252 & 240 & 251 & 241 & 251 & 241 & 224 & 248 & 249 \\
\hline NET SALE TOHL\&P & & 920 & 910 & 910 & 900 & 900 & 900 & 460 & 900 & 900 \\
\hline NET SALE TO HL\&P & & 1184 & 1192 & 1200 & 1207 & 1215 & 1223 & 1227 & 1234 & 1242 \\
\hline & & & $\cdots$ & & & & & & & \\
\hline & & & & & & & & & & \\
\hline \multicolumn{11}{|l|}{ BRINE } \\
\hline TO E-2-N & $P \mid-606$ & 500 & 500 & 500 & 500 & 500 & 500 & 500 & 500 & 500 \\
\hline TO E-1-N & P1.611 & 490 & 490 & 490 & 490 & 490 & 490 & 510 & 490 & 490 \\
\hline FROM E-1-N & PI.106 & 475 & 485 & 490 & 487 & 488 & 490 & 507 & 489 & 490 \\
\hline \multicolumn{11}{|l|}{ ISOBUTANE } \\
\hline IN $V-1-E$ & PG.130 & 56 & 57 & 56 & 58 & 59 & 58 & 46 & 60 & 60 \\
\hline OUT OF P-1-E & $\mathrm{PI} \cdot 105$ & 364 & 357 & 363 & 368 & 351 & 355 & 404 & 364 & 260 \\
\hline OUT OF E-1-N & $\overline{P 1.602}$ & 300 & 300 & 300 & 300 & 300 & 300 & 185 & 295 & 300 \\
\hline OUT OF E-2-N & $\overline{P G-114}$ & 280 & 285 & 280 & 280 & 285 & 280 & 185 & 280 & 280 \\
\hline OUT OF E-3-N & P1.615 & 300 & 300 & 300 & 300 & 300 & 300 & 190 & 300 & 300 \\
\hline INTO T-1-E & $\mathrm{P} \mid-107$ & 267 & 264 & 270 & 269 & 268 & 269 & 43 & 265 & 267 \\
\hline OUT OF T-1-E & $P \mid-108$ & 58 & 59 & 58 & 60 & 62 & 60 & 44 & 64 & 61 \\
\hline TO OA 102 & PG-104 & 60 & 60 & 60 & 65 & 70 & 65 & 380 & 70 & $\overline{65}$ \\
\hline FROM OA 102 & $\overline{P G-105}$ & 45 & 50 & 50 & 50 & 50 & 50 & 140 & 50 & 50 \\
\hline \multicolumn{11}{|c|}{ 年 } \\
\hline TO E-3-N & $\overline{P I .614}$ & 0.8 & 0.8 & 0.8 & 0.8 & 0.8 & 0.8 & 0 & 0.8 & $.5-1.0$ \\
\hline & & & & & & & & & & \\
\hline \multicolumn{11}{|l|}{ BAINE } \\
\hline TO E-2-N & $T 1.101$ & 297 & 297 & 297 & 297 & 296 & 297 & 297 & 297 & 298 \\
\hline TO E-1-N & TR-113 & 15 & & 216 & 15 & 0.5 & 0.5 & 216 & 215 & $\overline{215}$ \\
\hline FROM E-1.N & TR-102 & 15 & 1 & 151 & 15 & 0.5 & 0.5 & 152 & 152 & 157 \\
\hline & & & & & & & & & & \\
\hline & & & & & & & & & & \\
\hline & & & & & & & & & & \\
\hline & & & & & & & & & & \\
\hline & & & & & & & & & & \\
\hline
\end{tabular}


January $15-17,1990$

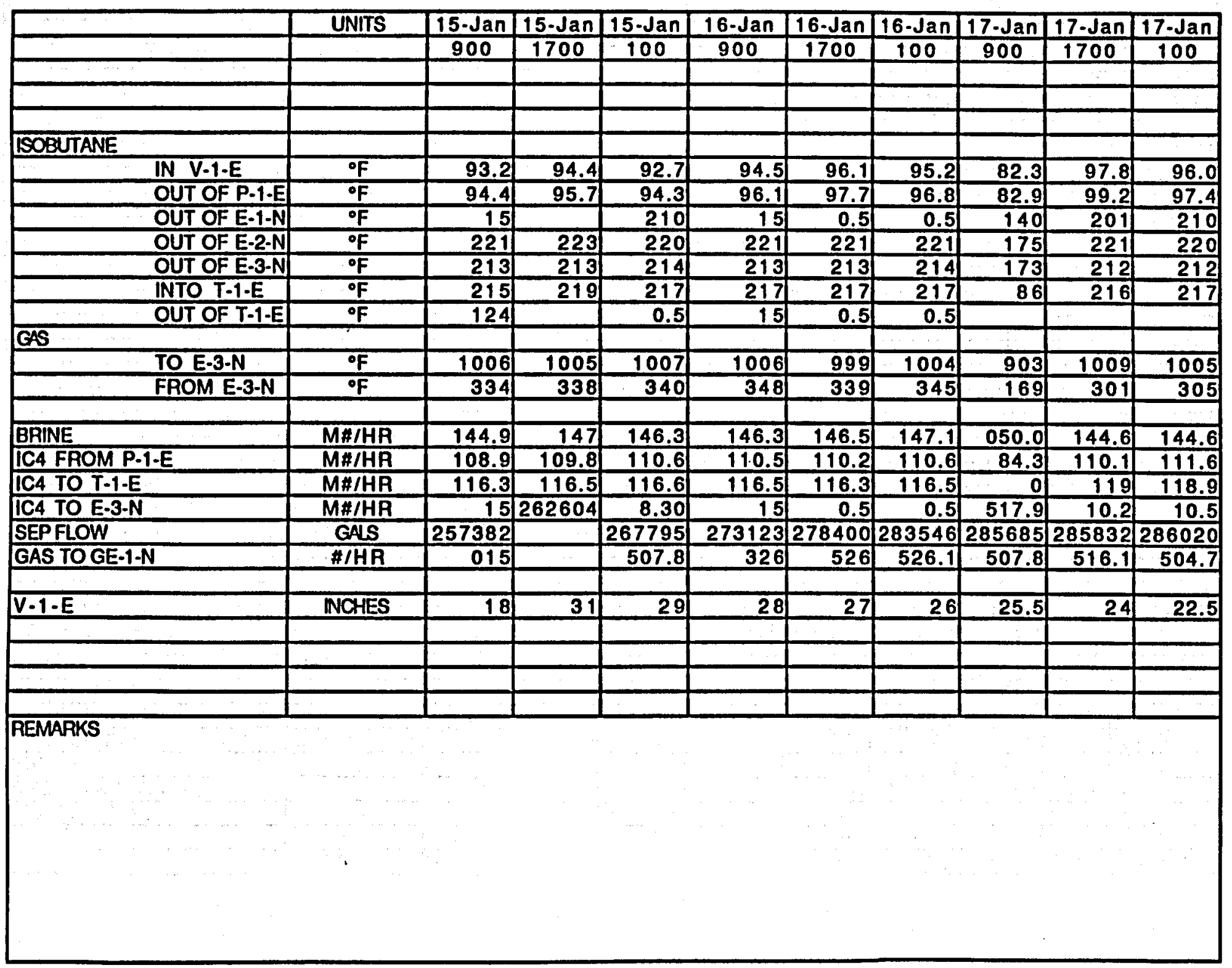


GEOPRESSURED HYBRID POWER SYSTEM - MANAGEMENT REPORT DATA

January 18-21, 1990

\begin{tabular}{|c|c|c|c|c|c|c|c|c|c|c|c|c|c|c|}
\hline & UNITS & INST.\# & 18-Jan & 18-Jan & 18-Jan & 19-Jan & 19-Jan & 19-Jan & 20-Jan & 20-Jan & 20-Jan & 21-Jan & 21-Jan & 21-Jan \\
\hline & & & 900 & 1700 & 100 & 900 & 1700 & 100 & 900 & 1700 & 100 & 900 & 1700 & 100 \\
\hline AMBIENT TEMP & ${ }^{\circ} \mathrm{F}$ & & 70 & 70 & 66 & 70 & 70 & 57 & 50 & 59 & 52 & 55 & $\begin{array}{r}61 \\
\end{array}$ & 48 \\
\hline WET BULB TEMP & of & & 69 & 68 & 66 & 68 & 69 & 57 & 49 & 52 & 48 & 47 & 50 & 45 \\
\hline POWEA & & & & & & & & & & & & & & \\
\hline T-1-N GROSS & $\mathrm{KW}$ & $J 1-100$ & 502 & 508 & 516 & 510 & 512 & 534 & 574 & 563 & 573 & 582 & 568 & 579 \\
\hline GE-1-N GROSS & $\overline{K W}$ & & 340 & 340 & 340 & 340 & 345 & 340 & 345 & 345 & 345 & 345 & 350 & 345 \\
\hline GE-2-N GROSS & $\mathrm{KW}$ & & 340 & 340 & 340 & 340 & 340 & 345 & 345 & 345 & 345 & 345 & 345 & 345 \\
\hline PARASITIC & $\mathrm{KW}$ & $J 1-101$ & 248 & 257 & 258 & 257 & 247 & 260 & 252 & 260 & 252 & 262 & 261 & 264 \\
\hline NET SALE TOHL\&P & $\mathbf{K W}$ & & 900 & 900 & 900 & 900 & 900 & 920 & 940 & 970 & 960 & 970 & 960 & 980 \\
\hline NET SALE TOHL\&P & FDG & & 1250 & 1258 & 1266 & 1274 & 1282 & 1290 & 1298 & 1307 & 1315 & 1323 & 1332 & 1341 \\
\hline & $\mathrm{KW} \cdot \mathrm{HR}$ & & & & & & & & & & & & & \\
\hline & & & & & & & & & & & & & & \\
\hline BRINE & & & & & & & & & & & & & $\because$ & \\
\hline TO E-2-N & PSIG & $\mathrm{P} / .606$ & 500 & 496 & 500 & 500 & 501 & 500 & 501 & 501 & 500 & 500 & 501 & 500 \\
\hline TO E-1-N & PSIG & PI-611 & 495 & 495 & 490 & 490 & 495 & 495 & 492 & 491 & 490 & 490 & 492 & 490 \\
\hline FROM E-1-N & PSIG & $\mathrm{P} /-106$ & 488 & 487 & 490 & 485 & 488 & 486 & 486 & 488 & 487 & 487 & 487 & 485 \\
\hline ISOBUTANE & & & $\therefore$ & & & & & & & & & & & \\
\hline IN $V \cdot 1-E$ & PSIG & PG-130 & 61 & 60 & 60 & 60 & 60 & 54 & 49 & 52 & 49 & 49 & 50 & 48 \\
\hline OUT OF P-1-E & PSIG & $P 1-105$ & 361 & 359 & 350 & 350 & 357 & 349 & 352 & 348 & 352 & 345 & 356 & 338 \\
\hline OUT OF E-1-N & PSIG & PI.602 & 295 & 300 & 300 & 300 & 300 & 300 & 299 & 300 & 300 & 302 & 305 & 300 \\
\hline OUT OF E-2-N & PSIG & PG.114 & 280 & 280 & 280 & 280 & 280 & 280 & 283 & 285 & 280 & 283 & 285 & 285 \\
\hline OUT OF E-3-N & PSIG & PI.615 & 300 & 300 & 300 & 300 & 295 & 300 & 300 & 301 & 300 & 300 & 300 & 300 \\
\hline INTO T-1.E & PSIG & $P \mid-107$ & 265 & 267 & 216 & 268 & 268 & 267 & 269 & 270 & 270 & 269 & 271 & 270 \\
\hline OUT OF T-1-E & PSIG & $P \mid-108$ & 63 & 63 & 61 & 62 & 63 & 55 & 52 & 54 & 51 & 51 & 53 & 50 \\
\hline TO OR 102 & PSIG & PG-104 & 65 & 65 & 65 & 65 & 69 & 60 & 56 & 52 & 55 & 54 & 55 & 55 \\
\hline FAOM OA 102 & PSIG & PG-105 & 50 & 50 & 50 & 50 & 51 & 45 & 41 & 42 & 40 & 40 & 45 & 40 \\
\hline GAS & & & & & & & & & & & & & & \\
\hline TO E-3-N & PSIG. & PI.614 & 0.8 & 0.8 & $.5-1.0$ & 0.8 & 0.8 & 0.8 & 0.7 & 0.9 & 0.9 & 0.9 & 0.9 & 0.9 \\
\hline & & & & & & & & & & & & & & \\
\hline BAINE & & & & & & & & & & & & & & \\
\hline TO E-2-N & ${ }^{\circ} \mathrm{F}$. & TI-101 & 298 & 297 & 297 & 297 & 297 & 297 & 297 & 298 & 298 & 297 & 298 & 298 \\
\hline TOE-1-N & ${ }^{\circ} \mathrm{F}$ & TR-113 & 214.3 & 215 & 215 & 201 & 212 & 216 & 216 & 217 & 216 & 216 & 217 & 216 \\
\hline FAOM E-1-N & $\circ \mathrm{F}$ & TR.102 & 152 & 148 & 152 & 152 & 150 & 147 & 147 & 149 & 147 & 147 & 151 & 146 \\
\hline & & & & & & & & & & & & & & \\
\hline & & & & & & & & & & & & & & \\
\hline & & & & & & & & & & & & & & \\
\hline & & & & & & & & & & & & & & \\
\hline & & & & & & & & & & & & & & \\
\hline
\end{tabular}




\section{GEOPRESSURED HYBRID POWER SYSTEM - MANAGEMENT REPORT DATA}

January 18.21, 1990

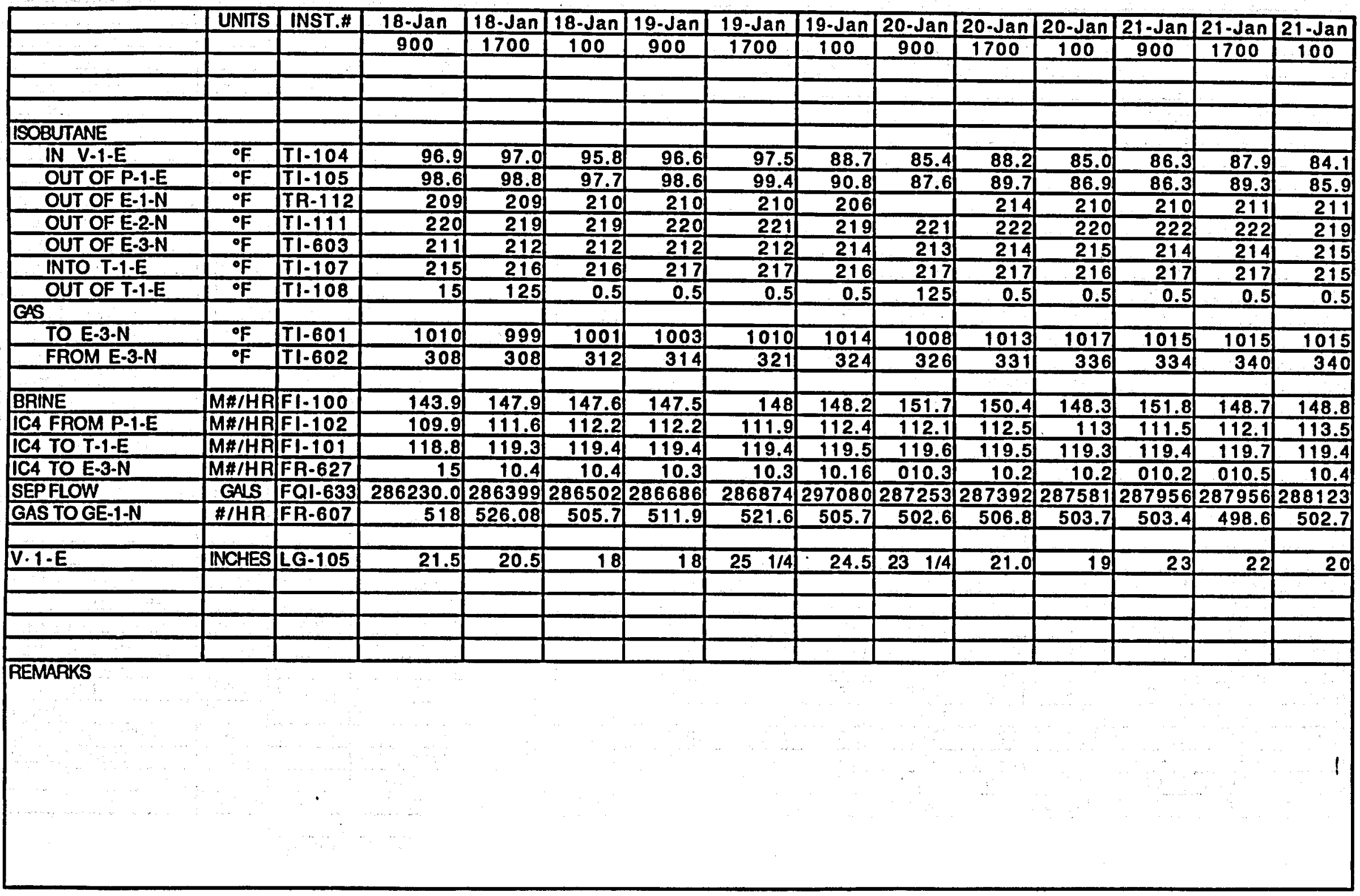


January 22-24, 1990

\begin{tabular}{|c|c|c|c|c|c|c|c|c|c|c|}
\hline & INST.\# & 22-Jan & 22-Jan & 22-Jan & 23-Jan & 23-Jan & 23-Jan & 24-Jan & 24-Jan & 24-Jan \\
\hline & & 900 & 1700 & 100 & 900 & 1700 & 100 & 900 & 1700 & 100 \\
\hline AMBIENT TEMP & & 56 & 62 & 53 & 65 & 68 & 67 & 73 & 71 & 56 \\
\hline WET BULB TEMP & & 52 & 54 & 52 & 63 & 66 & 66 & 71 & 69 & 48 \\
\hline \multicolumn{11}{|l|}{ POWEA } \\
\hline T-I-N GROSS & $J 1-100$ & 575 & 16 & 1 & 1 & 1 & 1 & 1 & 2 & 2 \\
\hline GE-1-NGROSS & & 340 & 345 & 345 & 345 & 340 & 340 & 340 & 340 & 340 \\
\hline GE-2-NGROSS & & 340 & 345 & 345 & 345 & 340 & 340 & 340 & 340 & 350 \\
\hline PARASITIC & $\$ 1.101$ & 252 & 16 & 6 & 6 & 16 & 16 & 18 & 8 & 7 \\
\hline NET SALE TO HL\&P & & 940 & 640 & 610 & 630 & 620 & 625 & 620 & 620 & 640 \\
\hline NET SALE TOHL\&P & & 1349 & 1354 & 1360 & 1365 & 1371 & 1376 & 1381 & 1387 & 1392 \\
\hline & & & & & & & & & $\therefore$ & \\
\hline & & & & & & & & & & \\
\hline \multicolumn{11}{|l|}{ BRINE } \\
\hline TO E-2-N & P/.606 & 500 & 510 & 510 & 510 & 510 & 510 & 510 & 510 & 510 \\
\hline TO E-1-N & $\mathrm{P} \mid-611$ & 490 & 520 & 520 & 515 & 520 & 520 & 515 & 518 & 515 \\
\hline FROM E-1-N & PI-106 & 486 & 515 & 519 & 518 & 520 & 519 & 516 & 518 & 515 \\
\hline \multicolumn{11}{|l|}{ ISOBUTANE } \\
\hline IN V-1-E & PG-130 & 51 & 30 & 27 & 28 & 34 & 34 & 35 & 34 & 33 \\
\hline OUT OF P.1-E & $P \mid-105$ & 342 & 35 & 27 & 31 & 36 & 33 & 35 & 35 & 27 \\
\hline OUT OF E-1.N & P1-602 & 305 & 45 & 25 & 30 & 30 & 30 & 35 & 35 & 25 \\
\hline OUT OF E-2.N & PG.114 & 285 & 30 & 25 & 25 & 25 & 30 & 35 & 35 & 25 \\
\hline OUT OF E-3-N & $P \mid-615$ & 300 & 54 & 55 & 55 & 50 & 40 & 35 & 35 & 40 \\
\hline INTO T.1.E & P1-107 & 271 & -24 & -24 & -24 & .24 & -24 & -24 & -24 & .24 \\
\hline OUT OF T-1.E & $P \mid-108$ & 53 & -11 & -11 & -11 & -11 & -11 & -11 & .11 & -11 \\
\hline TO. OA 102 & PG.104 & 55 & 30 & 30 & 30 & 25 & 25 & 35 & 40 & 40 \\
\hline FROM OR 102 & PG-105 & 40 & 20 & 20 & 15 & 35 & 35 & 25 & 25 & 25 \\
\hline \multicolumn{11}{|c|}{ 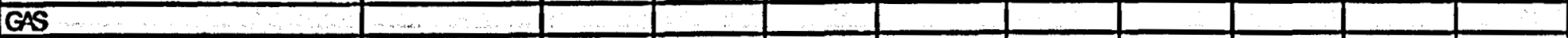 } \\
\hline TO-E-3-N & $\mathrm{P} \mid-614$ & 0.8 & 0.5 & 0.6 & 0.5 & 0.6 & 0.6 & 0.6 & 0.6 & 0.5 \\
\hline & & & & & & & & & & \\
\hline \multicolumn{11}{|l|}{ BRINE } \\
\hline TO E-2-N & $T /-101$ & 298 & 231 & 181 & 160 & 의 & 140 & 132 & 128 & 127 \\
\hline TO E-1-N & TR-113 & & 0.5 & 0.5 & 15 & 0.5 & 0.5 & 15 & 15 & 0.5 \\
\hline FROM E-1-N & TR-102 & & 0.5 & 0.5 & 15 & 0.5 & 0.5 & 15 & 15 & 0.5 \\
\hline & & & & & & & & & & \\
\hline & & & & & & & & & & \\
\hline & & & & & & & & & & \\
\hline & & & & & & & & & & \\
\hline & & & & & & & & & & \\
\hline
\end{tabular}


January 22.24, 1990

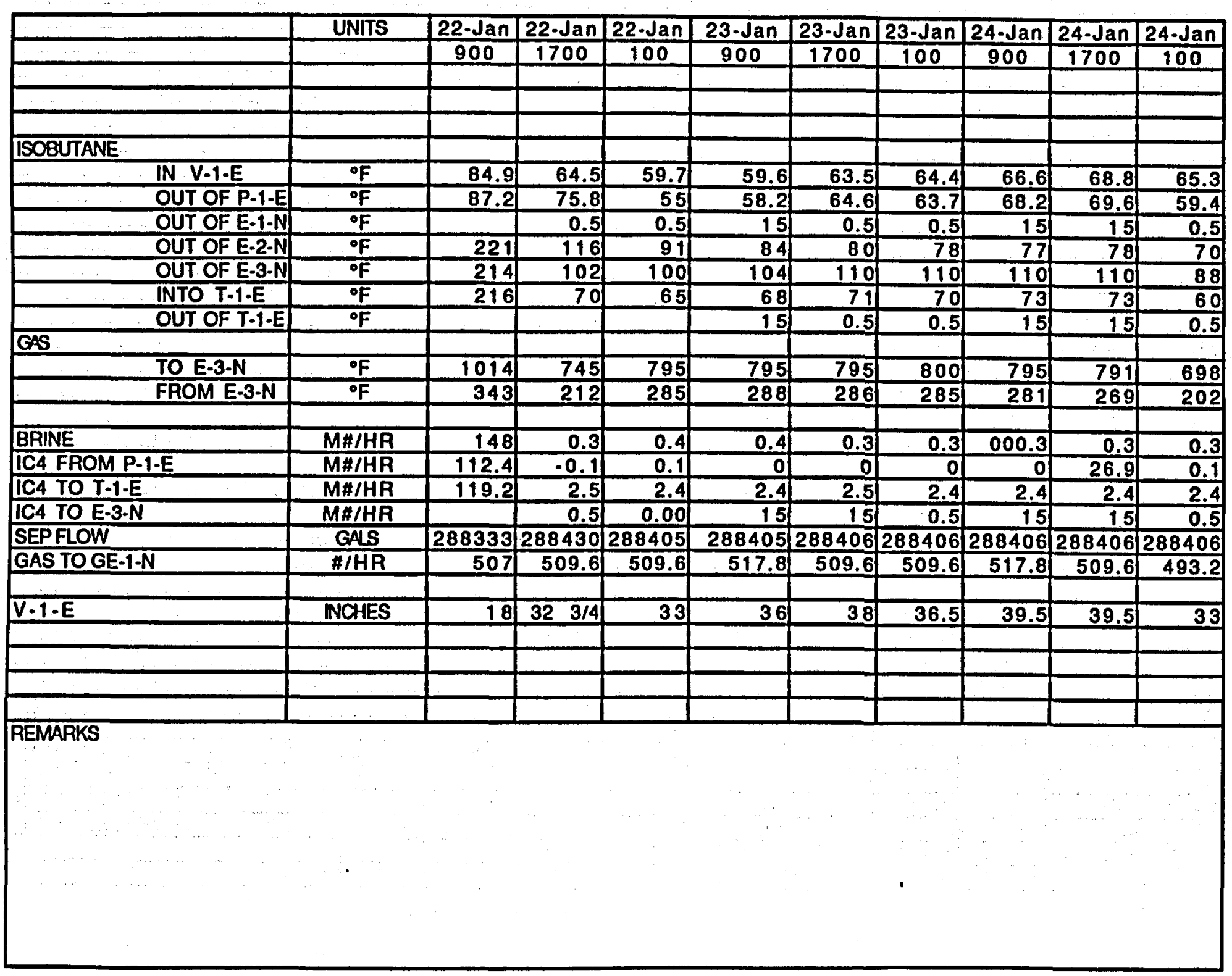


GEOPRESSURED HYBRID POWER SYSTEM - MANAGEMENT REPORT DATA

January 25-28, 1990

\begin{tabular}{|c|c|c|c|c|c|c|c|c|c|c|c|c|c|c|}
\hline - & UNITS & INST.\# & $25 \cdot \mathrm{Jan}$ & 25-Jan & 25-Jan & 26-Jan & 26-Jan & 26-Jan & 27-Jan & $27-J a n$ & 27-Jan & 28-Jan & 28-Jan & 28-Jan \\
\hline & & & 900 & 1700 & 100 & 900 & 1700 & 100 & 900 & 1700 & 100 & 900 & 1700 & 100 \\
\hline AMBIENT TEMP & ${ }^{\circ} \mathrm{F}$ & & 48 & 59 & 41 & 56 & 61 & 58 & 64 & 67 & 64 & 64 & 65 & 47 \\
\hline WET BULB TEMP & ० $\mathrm{F}$ & & 42 & 45 & 37 & 50 & 52 & 55 & 60 & 62 & 62 & 63 & 66 & 49 \\
\hline \multicolumn{15}{|l|}{ POWER } \\
\hline T-1-N GROSS & $\mathrm{KW}$ & $J 1-100$ & 2 & 1 & 1 & 1 & 1 & 1 & 1 & 0 & 0 & 0 & 1 & 1 \\
\hline GE.1.NGROSS & $\mathrm{KW}$ & & 340 & 345 & 350 & 350 & 345 & 350 & 350 & 350 & 350 & 350 & 345 & 350 \\
\hline GE-2.N GHOSS & $\mathrm{KW}$ & & 350 & 345 & 350 & 350 & 345 & 350 & 350 & 350 & 350 & 350 & 345 & 350 \\
\hline PARASITIC & $\mathrm{KW}$ & 31.101 & 7 & 11 & 17 & 6 & 6 & 17 & 6 & 23 & 16 & 16 & 16 & 6 \\
\hline NET SALE TOHIL\&P & $\mathrm{KW}$ & & 620 & 635 & 620 & 640 & 640 & 640 & 640 & 640 & 640 & 640 & 615 & 20 \\
\hline NET SALE TO HLBP & FIOG & & 1397 & 1403 & 1408 & 1414 & 1419 & 1425 & 1430 & 1436 & 1441 & 1447 & 1452 & 1458 \\
\hline$\therefore$ & KW-HA & & & & $\therefore$ & $\therefore$ & & & & $\because \cdots$ & $\therefore$ & & & $\therefore$ \\
\hline & & & & & & & & & & & & & & \\
\hline BAINE & & & 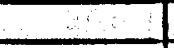 & & 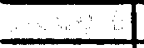 & $2 \div$ & & & & & & & 2 & \\
\hline TO E.2.N & PSIG & $\mathrm{P} / .606$ & 505 & 510 & 510 & 510 & 511 & 510 & 510 & 515 & 510 & 510 & 511 & 510 \\
\hline TOE-1.N & PSIG & $P \mid-611$ & 515 & 322 & 520 & 520 & 521 & 520 & 520 & 520 & 520 & 520 & 521 & 520 \\
\hline FROM E-1.N & PSIG & $P 1-106$ & 517 & 512 & 515 & 517 & 514 & 516 & 520 & 520 & 520 & 520 & 516 & 516 \\
\hline \multicolumn{15}{|l|}{ ISOBUTANE } \\
\hline IN V-1-E & PSIG & PG.130 & 24 & 26 & 21 & 20 & 29 & 25 & 26 & 36 & 32 & 30 & 30 & 22 \\
\hline OUT OF P.1.E & PSIG & $P 1.105$ & 25 & 24 & 16 & 16 & 26 & 4 & 25 & 32 & 27 & 27 & 30 & 14 \\
\hline OUT OF E-1-N & PSIG & $P 1.602$ & 20 & 28 & 20 & 20 & 31 & 28 & 30 & 30 & 30 & 30 & 30 & 20 \\
\hline OUT OF E-2.N & PSIG & PG.114 & 20 & 28 & 20 & 20 & 30 & 28 & 30 & 0 & 28 & 30 & 28 & 20 \\
\hline OUT OF E-3.N & PSIG & Pl.615 & 35 & 38 & 40 & 35 & 32 & 35 & 35 & 35 & 35 & 35 & 35 & 35 \\
\hline INTO T.1.E & PSIG & PI.107 & -25 & 5 & .95 & .95 & .95 & .95 & .95 & .95 & .95 & -95 & -95 & .95 \\
\hline OUT OF T.1.E & PSIG & $P 1.108$ & -12 & 5 & .49 & -49 & -49 & .49 & .49 & .49 & .49 & .49 & 49 & .49 \\
\hline TO Of 102 & PSIG & PG-104 & 25 & 29 & 28 & 25 & 30 & 30 & 30 & 40 & 35 & 35 & 34 & 25 \\
\hline FROM OR 102 & PSIG & PG-105 & 15 & 18 & 12 & 0 & 11 & 20 & 20 & 30 & 25 & 25 & 22 & 15 \\
\hline \multicolumn{15}{|l|}{ GS } \\
\hline TO E-3.N & PSIG & 01.614 & 0.5 & 0.55 & 0.6 & 0.6 & 0.5 & 0.6 & 0.6 & 0.6 & 0.6 & 0.6 & 0.4 & 0.5 \\
\hline & & & & & & & & & & & & & & \\
\hline \multicolumn{15}{|l|}{ BAINE } \\
\hline TO E.2.N & ${ }^{\circ} \mathrm{F}$ & $T 1-101$ & 128 & 129 & 130 & 129 & 128 & 126 & 125 & 125 & 119 & 116 & 113 & 101 \\
\hline TO E-1.N & ${ }^{\circ} \mathrm{F}$ & TR.113 & 015 & & 0.5 & 015 & & 015 & 0.5 & 0.5 & 015 & 0.5 & & 015 \\
\hline FAOM E-1-N & ${ }^{\circ} \mathrm{F}$ & TR.102 & 015 & & 0.5 & 015 & & 015 & 0.5 & 0.5 & 015 & 0.5 & & 615 \\
\hline & & & & & & & & & & & & & & \\
\hline & & & & & & & & & & & & & & \\
\hline & & & & & & & & & & & & & & \\
\hline & & & & & & & & & & & & & & \\
\hline & & & & & & & & & & & & & & \\
\hline
\end{tabular}


GEOPRESSURED HYBRID POWER SYSTEM - MANAGEMENT REPORT DATA

January $25.28,1990$

\begin{tabular}{|c|c|c|c|c|c|c|c|c|c|c|c|c|c|c|}
\hline & UNITS & INST.\# & 25.Jan & 25-Jan & $25-\mathrm{Jan}$ & $26-\mathrm{Jan}$ & 26-Jan & 26-Jan & $27 . \mathrm{Jan}$ & $27 . \mathrm{Jan}$ & 27-Jan & $28-J a n$ & $28 \cdot J a n$ & $28 . \mathrm{Jan}$ \\
\hline & & & 900 & 1700 & 100 & 900 & 1700 & 100 & 900 & 1700 & 100 & 900 & 1700 & 100 \\
\hline & & & & & & & & & & & & & & \\
\hline & & & & & & & & & & & & & & \\
\hline & & & & & & & & & & & & & & \\
\hline \multicolumn{15}{|l|}{ ISOBUTANE } \\
\hline IN V.1.E & of & $T 1.104$ & 54.9 & 58.4 & 51.7 & 48.2 & 55.2 & 56.2 & 58.1 & 63.0 & 64.5 & 65.0 & 65.9 & $\overline{54.2}$ \\
\hline OUT OF P.1-E & ${ }^{\circ} \mathrm{F}$ & TI.105 & 48.7 & 55.4 & 44.7 & 46.7 & 72.9 & 55 & 60.9 & 69.5 & 62.9 & 62.9 & 62.5 & 48.3 \\
\hline OUT OF E-1.N & ${ }^{\circ} \mathrm{F}$ & TA-112 & 015 & & 5 & 015 & & 015 & 0.5 & 0.5 & 015 & 0.5 & & 015 \\
\hline OUT OF E-2.N & ${ }^{\circ} \mathrm{F}$ & $T 1.111$ & 60 & 66 & 58 & 55 & 76 & 67 & 69 & 76 & 71 & 69 & $\overline{71}$ & 59 \\
\hline OUT OF E-3.N & ${ }^{\circ} \mathrm{F}$ & $T 1.603$ & 67 & 75 & $\overline{85}$ & 91 & 101 & 94 & 100 & 102 & 86 & 86 & 87 & $\frac{64}{64}$ \\
\hline INTO T-1-E & ${ }^{\circ} \mathrm{F}$ & T1.107 & 51 & 66 & 44 & 60 & 70 & 69 & 68 & 74 & 71 & 71 & 63 & .30 \\
\hline OUT OF T.1.E & ${ }^{\circ} \mathrm{F}$ & $T 1.108$ & 015 & & 5 & 015 & & -53 & -53 & .52 & .54 & .54 & .54 & .54 \\
\hline \multicolumn{15}{|l|}{ GS } \\
\hline TO E-3.N & ${ }^{\circ} \mathrm{F}$ & $T 1.601$ & 732 & 742 & 800 & 804 & 753 & 792 & 794 & 748 & 727 & 736 & 780 & 716 \\
\hline FHOM E-3.N & ${ }^{\circ} \mathrm{F}$ & $\mathrm{TI} .602$ & 171 & 172 & 263 & 284 & 235 & 260 & 269 & 209 & 185 & 185 & 190 & 155 \\
\hline & & & & & & & & & & & & +1 & & \\
\hline BRINE & $M H / H N$ & $F 1.100$ & 0.4 & & 5 & 15 & 0 & 요 & 0 & 0 & $\underline{0}$ & 0 & 0 & .11 \\
\hline IC4 FИОМ P.1-E & $\overline{M H I H A}$ & $\overline{F 1.102}$ & 0.1 & .0 & 000.2 & .1 & 0 & 0 & 49.1 & 0 & $\overline{0}$ & 0 & .1 & .2 \\
\hline ICA TO T-1.E & $\overline{M A 1+1 \mathrm{R}}$ & $\mathrm{FI.101}$ & 2.2 & &. .1 &. .2 & 0.1 & 2 & 0.1 & .2 & .2 & 0.2 & .3 & .2 \\
\hline IC4 TO E-3.N & $\mathrm{MHIHN}$ & FA-627 & 015 & & 5 & 15.0 & & & $\begin{array}{r}0.5 \\
\end{array}$ & 0.5 & 015 & $\begin{array}{r}015 \\
\end{array}$ & 010.5 & 015 \\
\hline SEP FLOW & GNS & FQ1.633 & 288406 & 288406 & 288406 & 288406 & 288406 & 288406 & 288406 & 288406 & 288406 & 288409 & 287956 & $\overline{290296}$ \\
\hline GAS TO GE.1.N & $\# / H A$ & FA-607 & 501.4 & 501.4 & 509.6 & 509.6 & 501.4 & 509.6 & 509.6 & 509.6 & 509.6 & 509.6 & 498.6 & 509.6 \\
\hline \multirow[t]{4}{*}{$V \cdot 1 \cdot E$} & INCHIES & LG-105 & 32 & 37 & $32 \quad 1 / 4$ & 35 & 37 & 36.5 & 37 & 39.0 & 34 & 35 & 22 & $\overline{35.5}$ \\
\hline & & & & & & & & & & & & & & \\
\hline & & & & & & & & & & & & & & \\
\hline & & & & & & & & & & & & & & \\
\hline \multirow[t]{7}{*}{ HEMUIKS } & & & & & & 1 & & & & & & & & \\
\hline & & & & & & & & & & & & & & \\
\hline & & & & & & & & & & & . & & & \\
\hline & & & & & & 1 & & & & & & & & 1 \\
\hline & & & & & & $:$ & & & & & & & & \\
\hline & & & & & & ? & & & & & & & & \\
\hline & & & & & & $i$ & & & & & & & & \\
\hline
\end{tabular}


January 29.31, 1990

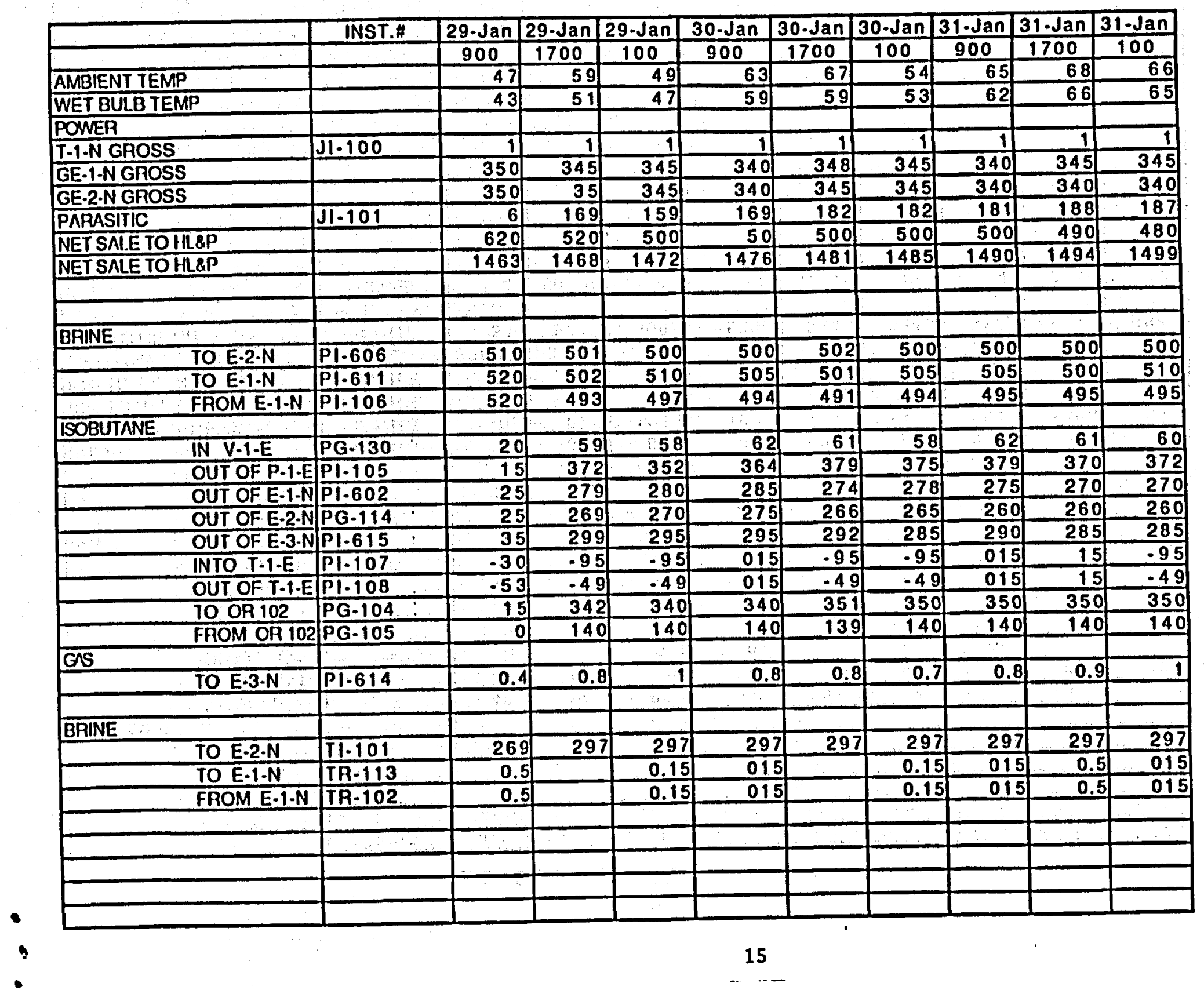


January 29.31, 1990

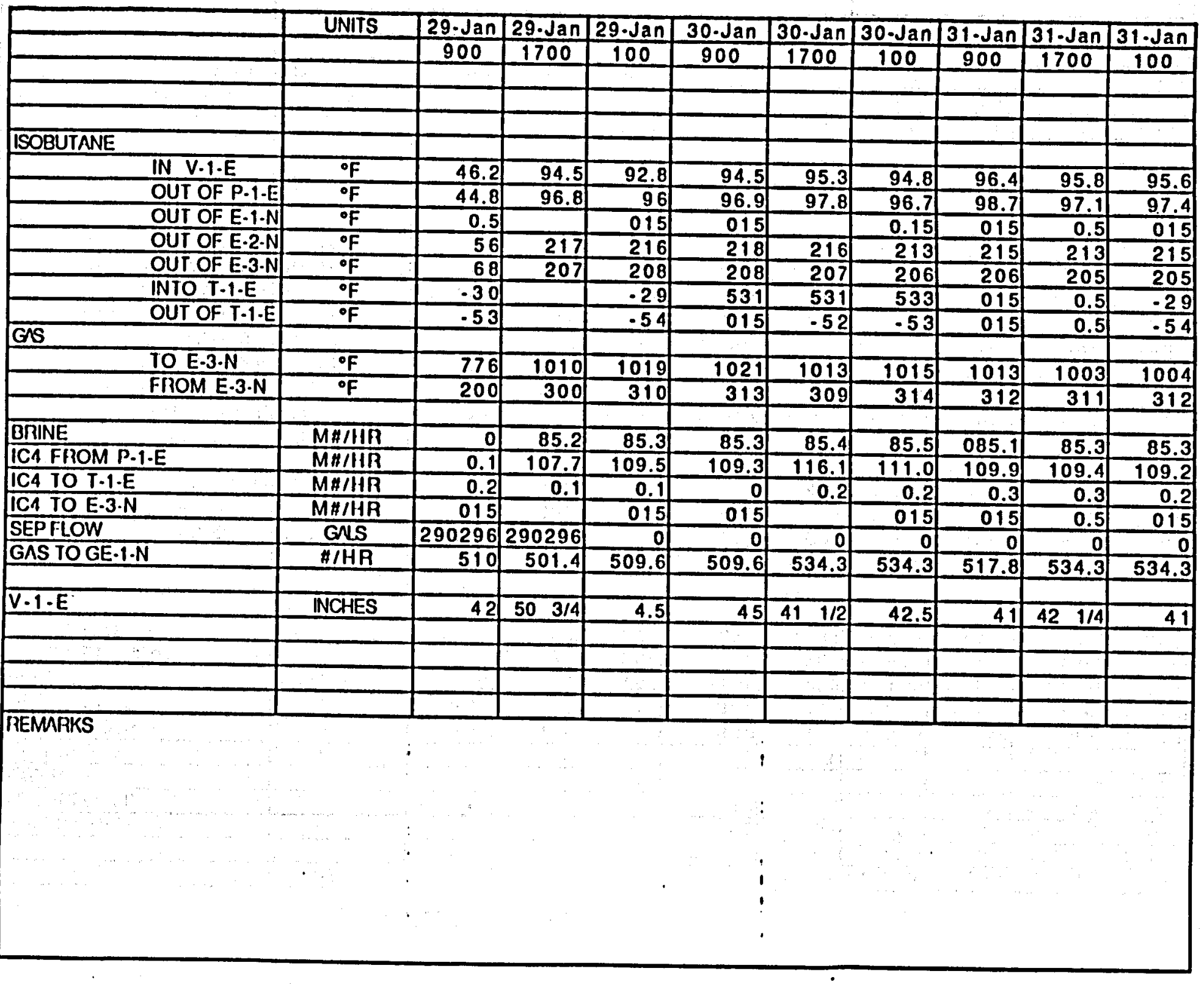


February 1-7, 1990

\begin{tabular}{|c|c|c|c|c|c|c|c|c|c|c|c|c|c|c|}
\hline & UNITS & INST.\# & 1-Feb & 1.Feb & 1.Fob & 2-Feb & 2-Feb & 2-Feb & 3-Fob & 6.Fob & 6-Fob & 7.Feb & 7.Fob & 7.Feb \\
\hline & & & 900 & 1700 & 100 & 900 & 1700 & 100 & 900 & 1700 & 100 & 900 & 1700 & 100 \\
\hline AMBIENT TEMP & ० $\mathrm{F}$ & & 68 & 70 & 60 & 68 & 70 & 68 & 70 & 64 & 57 & 61 & 64 & 62 \\
\hline WET BULB TEMP & oF & & 67 & 63 & 59 & 68 & 69 & 68 & 70 & 63 & 57) & 97 & 61 & 62 \\
\hline \multicolumn{15}{|l|}{ POWEA } \\
\hline T-1-N GROSS & $\mathrm{KW}$ & $J 1.100$ & 1 & 1 & 1 & 1 & 1 & 1 & 1 & 1 & 1 & 1 & 1 & 1 \\
\hline GE-1-N GROSS & $\mathbf{K W}$ & & 340 & 340 & 340 & 340 & 340 & 340 & 335 & 340 & 340 & 340 & 345 & 340 \\
\hline GE-2-N GROSS & KW & & 345 & 340 & 340 & 340 & 340 & 340 & 335 & 340 & 340 & 340 & 345 & 340 \\
\hline PARASITIC & KW & J1-101 & 188 & 177 & 178 & 177 & 180 & 176 & 187 & 171 & 173 & 171 & 17 & 180 \\
\hline NET SALE TOHL\&P & $\mathrm{KW}$ & & 520 & 500 & 480 & 500 & 490 & 490 & 495 & 510 & 500 & 500 & 510 & 500 \\
\hline \multirow{3}{*}{ NET SALE TOHL\&P } & $\mathbf{A D G}$ & & 1503 & 1507 & 1511 & 1516 & 1520 & 1526 & 1528 & 1532 & 1535 & 1540 & 1544 & 1548 \\
\hline & $\mathrm{KW} \cdot \mathrm{HA}$ & & & $\cdots$ & 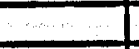 & & 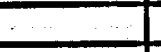 & & & & & & & \\
\hline & & \multicolumn{13}{|c|}{ 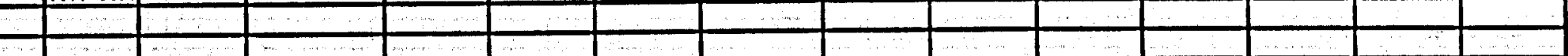 } \\
\hline BRINE & & & & & & & & & & & & & & \\
\hline TO E-2-N & PSIG & PI.606 & 500 & 500 & 500 & 500 & 505 & 500 & 504 & 500 & 500 & 500 & 505 & 500 \\
\hline TO E-1-N & PSIG & P1.611 & 505 & 500 & 510 & 505 & 505 & 510 & 518 & 500 & 500 & 505 & 505 & 500 \\
\hline FROM E-1-N & PSIG & $P I-106$ & 495 & 495 & 495 & 494 & 496 & 500 & 497 & 494 & 510 & 495 & 496 & 500 \\
\hline \multicolumn{15}{|l|}{ ISOBUTANE } \\
\hline IN V-1-E & PSIG & PG-130 & 61 & 59 & 58 & 62 & 64 & -58 & 61 & 60 & 58 & 60 & 61 & 60 \\
\hline OUT OF P.1-E & PSIG & $\mathrm{P} / 1.105$ & 371 & 366 & 361 & 362 & 430 & 437 & 439 & 429 & 414 & 360 & 358 & 365 \\
\hline OUT OF E-1-N & PSIG & $P 1.602$ & 275 & 275 & 275 & 275 & 270 & 270 & 277 & 270 & 280 & 305 & 305 & 305 \\
\hline OUT OF E-2-N & PSIG & PG.114 & 265 & 260 & 260 & 262 & 260 & 260 & 266 & 270 & 265 & 270 & 267 & 265 \\
\hline OUT OF E-3-N & PSIG & $\mathrm{PI}-615$ & 285 & 285 & 285 & 275 & 265 & 265 & 270 & 270 & 275 & 275 & 271 & 270 \\
\hline INTO T-1.E & PSIG & $P \mid-107$ & $\cdots$ & $\cdots$ & $\cdots$ & $\cdots$ & $\cdots$ & $\cdots$ & $\cdots$ & $\cdots$ & $\cdots$ & $\cdots$ & $\cdots$ & $\cdots$ \\
\hline OUT OF T.1-E & PSIG & $\mathrm{Pl} \cdot 108$ & $\cdots$ & $\cdots$ & $\cdots$ & $\cdots$ & $\cdots$ & $\cdots$ & $\cdots$ & $\cdots$ & $\cdots$ & $\cdots$ & $\therefore$ & $\cdots$ \\
\hline TO OR 102 & PSIG & PG-104 & 350 & 340 & 340 & 350 & 60 & 60 & 60 & 60 & 60 & 330 & 3.32 & 330 \\
\hline FROM OR 102 & PSIG & PG-105 & 140 & 150 & 150 & 150 & 60 & 60 & 60 & 60 & 55 & 140 & 145 & 145 \\
\hline \multicolumn{15}{|l|}{ GAS } \\
\hline TO E-3-N & PSIG & $\mathrm{PI}-614$ & 0.9 & 0.9 & 0.9 & 0.6 & 0.9 & 1.0 & 0.9 & 0.9 & 1.0 & 1.0 & 1.0 & 1.0 \\
\hline & & & & & & & & & & & & & 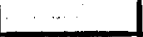 & \\
\hline \multicolumn{15}{|l|}{ BAINE } \\
\hline TO E-2-N & OF & $T I .101$ & 297 & 297 & 298 & 129 & 297 & 297 & 298 & 298 & 298 & 297 & 297 & 297 \\
\hline TO E-1-N & of & TA-113 & $\cdots$ & $\cdots$ & $\cdots$ & $\cdots$ & $\cdots$ & $\cdots$ & $\cdots$ & $\cdots$ & $\cdots$ & $\cdots$ & $\cdots$ & $\cdots$ \\
\hline FROM E-1-N & OF & TA-102 & $\cdots$ & $\because$ & $\cdots$ & $\cdots$ & $\cdots$ & $\cdots$ & $\cdots$ & $\cdots$ & $\cdots$ & $\cdots$ & $\cdots$ & $\ldots$ \\
\hline & & & & & - & & & & & & & & & \\
\hline & & & & & & & & & & & & & & \\
\hline & & & & & & & & & & & & & & \\
\hline & & & & & & & & & & & & & & \\
\hline & & & & & & & & & & & & & & \\
\hline
\end{tabular}




\section{GEOPRESSURED HYBRID POWER SYSTEM - MANAGEMENT REPORT DATA}

February 1.7, 1990

\begin{tabular}{|c|c|c|c|c|c|c|c|c|c|c|c|c|c|c|}
\hline & UNITS & INST.\# & 1-Feb & 1.Feb & 1-Feb & 2-Feb & 2-Fab & 2.Fob & 3.Fob & 6-Feb & 6.Fob & 7.Fob & 7-Feb & $7 \cdot \mathrm{Feb}$ \\
\hline & & & 900 & 1700 & 100 & 900 & 1700 & 100 & 900 & 1700 & 100 & 900 & 1700 & 100 \\
\hline & & & & & & & & & & & & & & \\
\hline & & & & & & & & & & & & & & \\
\hline & & & & & & & & & & & & & & \\
\hline \multicolumn{15}{|l|}{ ISOBUTANE } \\
\hline IN V-1-E & oF & $T 1-104$ & 97.0 & 94.6 & 93.9 & 98.3 & 99.7 & 95.8 & 97.3 & 96.8 & 93.9 & 95.0 & $\overline{96.2}$ & $\overline{96.3}$ \\
\hline OUT OF P-1-E & ${ }^{\circ} \mathrm{F}$ & $T 1-105$ & 98.6 & 96.2 & 95.4 & 100.8 & 101.9 & 98.3 & 100.1 & 98.2 & 96.7 & 96.7 & 97.9 & 97.9 \\
\hline OUT OF E-1-N & ${ }^{\circ} \mathbf{F}$ & TR.112 & $\cdots$ & $\cdots$ & $\cdots$ & $\ldots$ & $\ldots$ & $\cdots$ & $\cdots$ & $\ldots$ & $\cdots$ & $\cdots$ & $\cdots$ & $\ldots$ \\
\hline OUT OF E-2-N & of & $T 1.111$ & 214 & 213 & 216 & 214 & 213 & 216 & 213 & 216 & 214 & 213 & 214 & 213 \\
\hline OUT OF E-3-N & of & T1.603 & 205 & 205 & 205 & 205 & 205 & 204 & 206 & 205 & 208 & 206 & 207 & 206 \\
\hline INTO T-1.E & of & $T 1.107$ & $\cdots$ & $\cdots$ & $\ldots$ & $\cdots$ & $\cdots$ & $\cdots$ & $\cdots$ & $\ldots$ & $\ldots$ & $\ldots$ & $\cdots$ & $\cdots$ \\
\hline OUT OF T-1-E & of & $T 1-108$ & $\cdots$ & $\ldots$ & $\cdots$ & $\cdots$ & $\ldots$ & $\cdots$ & $\cdots$ & $\ldots$ & $\cdots$ & $\ldots$ & $\cdots$ & $\ldots$ \\
\hline \multicolumn{15}{|l|}{ GAS } \\
\hline TO E-3-N & of & Tl.601 & 1015 & 1002 & 1010 & 1011 & 1007 & 1007 & 1010 & 1016 & 1019 & 1012 & 1013 & 1018 \\
\hline \multirow[t]{2}{*}{ FROM E-3-N } & ${ }^{\circ} \mathrm{F}$ & $T 1.602$ & 319 & 314 & 318 & 320 & 318 & 320 & 323 & 285 & 294 & 295 & 297 & 302 \\
\hline & & & & & & & & & & & & & & \\
\hline BAINE & M\#/HA & $F \mid \cdot 100$ & 85.2 & 84.7 & 85.3 & 85.2 & 85.2 & 85.0 & 85.2 & 87 & 87.2 & 86.9 & 872 & 86.8 \\
\hline IC4 FROM P-1-E & $\overline{M \# / H A}$ & $F 1.102$ & 110.4 & 109.7 & 108.7 & 109.6 & 68.2 & 68.2 & 67 & 107.5 & 54.9 & 112.9 & 112.4 & 112.3 \\
\hline IC4 TO T-1-E & $M \# I H A$ & FI.101 & 0.2 & .3 & .3 & .2 & .3 & .2 & 0.2 & .3 & .2 & 0.3 & .3 & .3 \\
\hline IC4 TO E-3-N & M\#/HA & FR-627 & $\ldots$ & $\ldots$ & $\cdots$ & $\ldots$ & $\cdots$ & $\ldots$ & $\cdots$ & $\cdots$ & $\cdots$ & $\cdots$ & $\cdots$ & $\cdots$ \\
\hline SEP FLOW & GALS & FQl-633 & 0 & 0 & 0 & 0 & 0 & 0 & 0 & 0 & 0 & 0 & & 0 \\
\hline \multirow[t]{2}{*}{ GAS TO GE-1-N } & \#/HR & FR.607 & 534.3 & 534.3 & 517.8 & 526.08 & 534.3 & 517.9 & 509.6 & 501.4 & 501.4 & 501.4 & 509.6 & 509.6 \\
\hline & & & & & & & & & & & & & & \\
\hline \multirow[t]{4}{*}{$V \cdot 1-E$} & INCHES & LG.105 & 41 & 42 & 40 & 40.5 & 40.5 & 42 & 41.5 & 49.0 & 48 & 47 & 39.75 & 42 \\
\hline & & & & & & & & & & & & & & \\
\hline & & & & & & & & & & & & & & \\
\hline & & & & & & & & & & & & & & \\
\hline & & & & & & & & & & & & & & \\
\hline \multicolumn{15}{|l|}{ REMARKS } \\
\hline & & & & & & & & & & & & & & \\
\hline & & & & & & & & & & & & & & \\
\hline & & & & & & & & & & & & & & \\
\hline & & & & & & & & & & & & & & \\
\hline
\end{tabular}


February 1.7 1990

\begin{tabular}{|c|c|c|c|c|c|c|c|c|c|c|}
\hline & INST.\# & 29-Jan & 29.Jan & 29-Jan & 30.Jan & 30-Jan & 30-Jan & 31-Jan & 31-Jan & 31-Jan \\
\hline & & \begin{tabular}{|l|}
900 \\
\end{tabular} & 1700 & 100 & 900 & 1700 & 100 & 900 & 1700 & 100 \\
\hline AMBIENT TEMP & & 47 & 59 & 49 & 63 & 67 & 54 & 65 & 68 & 66 \\
\hline WET BULB TEMP & & 43 & 51 & 471 & 59 & 59 & 53 & 62 & 66 & 65 \\
\hline \multicolumn{11}{|l|}{ POWEA } \\
\hline T-1-N GROSS & $J 1.100$ & 1 & 1 & 1 & 1 & 1 & 1 & 1 & 1 & 1 \\
\hline GE-1-N GROSS & & 350 & 345 & 345 & 340 & 348 & 345 & 340 & 345 & 345 \\
\hline GE-2-N GROSS & & 350 & 35 & 345 & 340 & 345 & 345 & 340 & 340 & 340 \\
\hline PARASTIIC & J1-101 & 6 & 169 & 159 & 169 & 182 & 182 & 181 & 188 & 187 \\
\hline NET SALE TOHLAP & & 620 & 520 & 500 & 50 & 500 & 500 & 500 & 490 & 480 \\
\hline NET SALE TOHLAP & & 1463 & 1468 & 1472 & 1476 & 1481 & 1485 & 1490 & 1494 & 1499 \\
\hline \multicolumn{11}{|l|}{ NET SALE TO HLAP } \\
\hline & & & & & & & & & & \\
\hline \multicolumn{11}{|l|}{ BAINE } \\
\hline TO E-2-N & $\mathrm{PI} 1.606$ & 510 & 501 & 500 & 500 & 502 & 500 & 500 & 500 & 500 \\
\hline TO-E-1.N & $\mathrm{Pl}-611$ & 520 & 502 & 510 & 505 & 501 & 505 & 505 & 500 & 510 \\
\hline FROM E-1-N & PI-106 & 520 & 493 & 497 & 494 & 491 & 494 & 495 & 495 & 495 \\
\hline \multicolumn{11}{|l|}{ ISOBUTANE } \\
\hline IN V.1-E & PG-130 & 20 & 59 & 58 & 62 & 61 & 58 & 62 & 61 & 60 \\
\hline OUT OF P-1-E & $\mathrm{P} 1.105$ & 15 & 372 & 352 & 364 & 379 & 375 & 379 & 370 & 372 \\
\hline OUT OF E-1-N & $\mathrm{P} 1.602$ & 25 & 279 & 280 & 285 & 274 & 278 & 275 & 270 & 270 \\
\hline OUT OF E-2-N & $P G-114$ & 25 & 269 & 270 & 275 & 266 & 265 & 260 & 260 & 260 \\
\hline OUT OF E-3-N & $\mathrm{PI}-615$ & 35 & 299 & 295 & 295 & 292 & 285 & 290 & 285 & 285 \\
\hline INTO T-1-E & $P \mid .107$ & .30 & -95 & -95 & 015 & .95 & .95 & 015 & 15 & .95 \\
\hline OUT OF T-1-E & $P \mid=108$ & .53 & -49 & -49 & 015 & .49 & .49 & 0.15 & 15 & -49 \\
\hline TO OR 102 & PG.104 & 15 & 342 & 340 & 340 & 351 & 350 & 350 & 350 & 350 \\
\hline FROM OA 102 & PG.105 & 0 & 140 & 140 & 140 & 139 & 140 & 140 & 140 & 140 \\
\hline \multicolumn{11}{|c|}{ 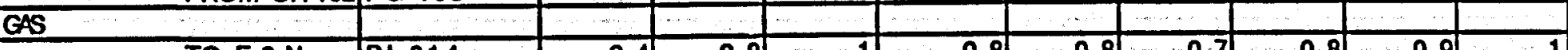 } \\
\hline TO E-3.N & $P 1.614$ & 0.4 & 0.8 & 1 & 0.8 & 0.8 & 0.7 & 0.8 & 0.9 & 1 \\
\hline \multirow{2}{*}{\multicolumn{11}{|c|}{ BRINE }} \\
\hline & & & & & & & & & & \\
\hline TO E-2-N & $T 1.101$ & 269 & 297 & 297 & 297 & 297 & 297 & 297 & 297 & 297 \\
\hline TO E-1-N & TR-113 & 0.5 & & 0.15 & 015 & & 0.15 & 015 & 0.5 & 015 \\
\hline FROM E-1-N & TR.102 & 0.5 & & 0.15 & 015 & & 0.15 & 015 & 0.5 & 015 \\
\hline & & & & & & & & & & \\
\hline & & & & & & & & & & \\
\hline & & & & & & & & & & \\
\hline & & & & & & & & & & \\
\hline & & & & & & & & & & \\
\hline
\end{tabular}


February 1.7 1990

\begin{tabular}{|c|c|c|c|c|c|c|c|c|c|c|}
\hline & UNITS & 29-Jan & 29-Jan & 29-Jan & $30-\mathrm{Jan}$ & 30-Jan & $30-\mathrm{Jan}$ & 31-Jan & 31-Jan & 31-Jan \\
\hline & & 900 & 1700 & 100 & 900 & 1700 & 100 & 900 & 1700 & 100 \\
\hline & & & & & & & & & & \\
\hline & & & & & & & & & & \\
\hline & & & & & & & & & & \\
\hline \multicolumn{11}{|l|}{ ISOBUTANE } \\
\hline IN V-1.E & ${ }^{\circ} \mathrm{F}$ & 46.2 & 94.5 & 92.8 & 94.5 & 95.3 & 94.8 & 96.4 & 95.8 & 95.6 \\
\hline OUT OF P-1-E & ${ }^{\circ} \mathrm{F}$ & 44.8 & 96.8 & 96 & 96.9 & 97.8 & 96.7 & 98.7 & 97.1 & 97.4 \\
\hline OUT OF E-1-N & ${ }^{\circ} \mathrm{F}$ & 0.5 & & 015 & 015 & & 0.15 & 015 & 0.5 & 015 \\
\hline OUT OFE-2-N & ${ }^{\circ} \mathrm{F}$ & 56 & 217 & 216 & 218 & 216 & 213 & 215 & 213 & 215 \\
\hline OUT OF E-3-N & ${ }^{\circ} \mathrm{F}$ & 68 & 207 & 208 & 208 & 207 & 206 & 206 & 205 & 205 \\
\hline INTO T-1-E & ${ }^{\circ} \mathrm{F}$ & -30 & & .29 & 531 & 531 & 533 & 015 & 0.5 & -29 \\
\hline OUT OF T-1-E & ${ }^{\circ} \mathrm{F}$ & -53 & & -54 & 015 & .52 & .53 & 015 & 0.5 & .54 \\
\hline \multicolumn{11}{|l|}{ GAS } \\
\hline TO E-3-N & ${ }^{\circ} \mathrm{F}$ & 776 & 1010 & 1019 & 1021 & 1013 & 1015 & 1013 & 1003 & 1004 \\
\hline FROM E-3-N & ${ }^{\circ} \mathrm{F}$ & 200 & 300 & 310 & 313 & 309 & 314 & 312 & 311 & 312 \\
\hline BRINE & $\mathrm{M} \# / \mathrm{HR}$ & 0 & 85.2 & 853 & 85.3 & 85.4 & 85.5 & 0851 & 853 & 853 \\
\hline IC4 FROM P-1.E & $\mathrm{M \# /HR}$ & 0.1 & 107.7 & 109.5 & 109.3 & 116.1 & 111.0 & $\begin{array}{ll}00.1 \\
109.9\end{array}$ & $\frac{80.4}{109.4}$ & $\frac{85.3}{109.2}$ \\
\hline IC4 TO T-1-E & M\#/HA & 0.2 & 0.1 & 0.1 & 0 & 0.2 & 0.2 & 0.3 & 0.3 & 0.2 \\
\hline IC4 TO E-3-N & M\#/HR & 015 & & 015 & 015 & & 015 & 015 & 0.5 & 015 \\
\hline SEPFLOW & GAS & 290296 & 290296 & 0 & 0 & 0 & 0 & 0 & 0 & 0 \\
\hline \multirow[t]{2}{*}{ GAS TO GE-1-N } & \#/HA & 510 & 501.4 & 509.6 & 509.6 & 534.3 & 534.3 & 517.8 & 534.3 & 534.3 \\
\hline & & & & & & & & & & \\
\hline \multirow[t]{4}{*}{$V-1-E$} & INCHES & 42 & $50 \quad 3 / 4$ & 4.5 & 45 & $41 \quad 1 / 2$ & 42.5 & 41 & $\begin{array}{|ll|}42 & 1 / 4 \\
\end{array}$ & 41 \\
\hline & & & & & & & & & & \\
\hline & & & & & & & & & & \\
\hline & & & & & & & & & & \\
\hline \multicolumn{11}{|l|}{ REMARKS } \\
\hline & & & & & & & & & & \\
\hline & & & & & & & & & & \\
\hline & & & & & & & & & & \\
\hline & & & & & & & & & & \\
\hline & & & & & & & & & & \\
\hline
\end{tabular}


February 8.11, 1990

\begin{tabular}{|c|c|c|c|c|c|c|c|c|c|c|c|c|c|c|}
\hline & UNITS & INST.\# & 8-Feb & 8.Feb & B-Fob & 9-Feb & 9-Feb & 9-Fob & 10.Feb & 10-Feb & 10-Feb & $11 \cdot \mathrm{Fob}$ & 11-Fob & 11-Fob \\
\hline & & & 900 & 1700 & 100 & 900 & 1700 & 100 & 900 & 1700 & 100 & 900 & 1700 & 100 \\
\hline AMBIENT TEMP & ${ }^{\circ} \mathrm{F}$ & & 67 & 68 & 67 & 72 & 70 & 58 & 58 & 62 & 49 & 58 & 73 & 52 \\
\hline WET BULB TEMP & ${ }^{\circ} \mathrm{F}$ & & 67 & 68 & 67 & 70 & 69 & 54 & 54 & 55 & 49 & 55 & 59 & 52 \\
\hline \multicolumn{15}{|l|}{ POWER } \\
\hline T-1-N GROSS & $\mathrm{KW}$ & $\mathrm{J} 1.100$ & 1 & 2 & 0 & 0 & 1 & 1 & 1 & 1 & 1 & 1 & 1 & 1 \\
\hline GE-1-N GROSS & $\mathrm{KW}$ & & 340 & 340 & 340 & 340 & 340 & 340 & 350 & 340 & 340 & 350 & 340 & 350 \\
\hline GE-2-NGROSS & $\mathrm{KW}$ & 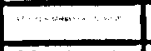 & 340 & 340 & 340 & 340 & 340 & 340 & 350 & 350 & 340 & 350 & 340 & 350 \\
\hline PARASITIC & $\mathrm{KW}$ & $\mathrm{J} 1.101$ & 180 & 193 & 193 & 187 & 187 & 189 & 183 & 181 & $\cdots 173$ & 182 & 181 & 182 \\
\hline NET SALE TOHL\&P & $\mathrm{KW}$ & & 500 & 485 & 480 & 480 & 485 & 500 & 520 & 510 & 480 & 520 & 510 & 500 \\
\hline NETSALE TOHL\&P & $\mathrm{ADG}$ & 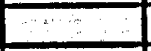 & 1553 & 1556 & 1561 & 1566 & 1570 & 1574 & 1579 & 1583 & 1587 & 1592 & 1596 & 1601 \\
\hline & $\mathrm{KW} \cdot \mathrm{HA}$ & $\cdots$ & & 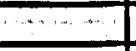 & & & $\ldots$ & & & & & & & \\
\hline & & & 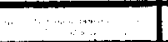 & & & $\cdots$ & & & & & & & & \\
\hline \multicolumn{15}{|l|}{ BRINE } \\
\hline TO E-2.N & PSIG & PI.606 & 500 & 500 & 510 & 505 & 500 & 500 & 510 & 500 & 500 & 500 & 500 & 505 \\
\hline TOE-1-N & PSIG & PI.611 & 505 & 500 & 510 & 510 & 500 & 500 & 520 & 500 & 510 & 510 & 500 & 510 \\
\hline FAOM E-1-N & PSIG & PI.106 & 495 & 497 & 500 & 490 & 492 & 500 & 495 & 495 & 500 & 491 & 490 & 500 \\
\hline \multicolumn{15}{|l|}{ ISOBUTANE } \\
\hline IN V.1-E & PSIG & PG-130 & 62 & 59 & 60 & 60 & 62 & 56 & 54 & 58 & 56 & 56 & 59 & 58 \\
\hline OUT OF P-1-E & PSIG & PI-105 & 368 & 365 & 364 & 370 & 365 & 349 & 350 & 336 & 349 & 357 & 366 & 365 \\
\hline OUT OF E-1-N & PSIG & PI-602 & 305 & 305 & 305 & 305 & -305 & 300 & 305 & -305 & 300 & 310 & 310 & 300 \\
\hline OUT OF E-2-N & PSIG & PG-114 & 265 & 270 & 265 & 265 & 265 & 260 & 265 & 270 & 265 & 270 & 270 & 260 \\
\hline OUT OF E-3-N & PSIG & $P \mid-615$ & 275 & 275 & 275 & 275 & 270 & 270 & 275 & 275 & 270 & 270 & 275 & 270 \\
\hline INTO T-1.E & PSIG & $P \mid-107$ & $\because$ & $\cdots$ & $\cdots$ & .095 & $-\cdots$ & $\cdots$ & $\cdots$ & $\cdots$ & $\cdots$ & $\cdots$ & -- & $\cdots$ \\
\hline OUT OF T-1.E & PSIG & $P \mid-108$ & --1 & $\cdots$ & $\ldots$ & .048 & $\cdots$ & $\cdots$ & $\cdots$ & $\cdots$ & $\cdots$ & $\cdots$ & $\ldots$ & $\cdots$ \\
\hline TO OA 102 & PSIG & PG-104 & 330 & 330 & 330 & 340 & 335 & 330 & 330 & 330 & 330 & 330 & 340 & 330 \\
\hline FAOM OR 102 & PSIG & PG-105 & 145 & 150 & 140 & 140 & 145 & 140 & 140 & 140 & 145 & 140 & 150 & 140 \\
\hline \multicolumn{15}{|l|}{ GAS } \\
\hline TO E-3-N & PSIG & P|.614 & 1.0 & 1.0 & 1.0 & 1.0 & 1.0 & 1.0 & 1.0 & 1.0 & 1.0 & 1.0 & 1.0 & 1.0 \\
\hline & & & & & & & & & & & & & & \\
\hline BRINE & & & 297 & & & & & & & & & & & \\
\hline TO E-2-N & ${ }^{\circ} \mathrm{F}$ & T1.101 & 297 & 297 & 297 & 297 & 297 & 297 & 297 & 298 & 298 & 298 & 297 & 298 \\
\hline TO E-1-N & OF & TR.113 & $\cdots$ & $\cdots$ & $\therefore$ & $\cdots$ & $\because-$ & $\cdots$ & $\cdots$ & $-\cdots$ & $\cdots$ & $\cdots$ & $-\cdots$ & $=\cdots$ \\
\hline FROM E-1-N & ${ }^{\circ} \mathrm{F}$ & TR. 102 & --1 & $\cdots$ & $\cdots$ & $\cdots$ & $\ldots$ & $\cdots$ & $\cdots$ & $\cdots$ & $\cdots$ & $\cdots$ & $\cdots$ & $-\cdots$ \\
\hline & $\cdots$ & & & & & & & & & & & & & \\
\hline & & & & & & & & & & & & & & \\
\hline & & & & & & & & & & & & & & \\
\hline & & & & & & & & & & & & & & \\
\hline & & & & & & & & & & & & & & \\
\hline
\end{tabular}




\section{GEOPRESSURED HYBRID POWER SYSTEM - MANAGEMENT REPORT DATA}

February 8.11, 1990

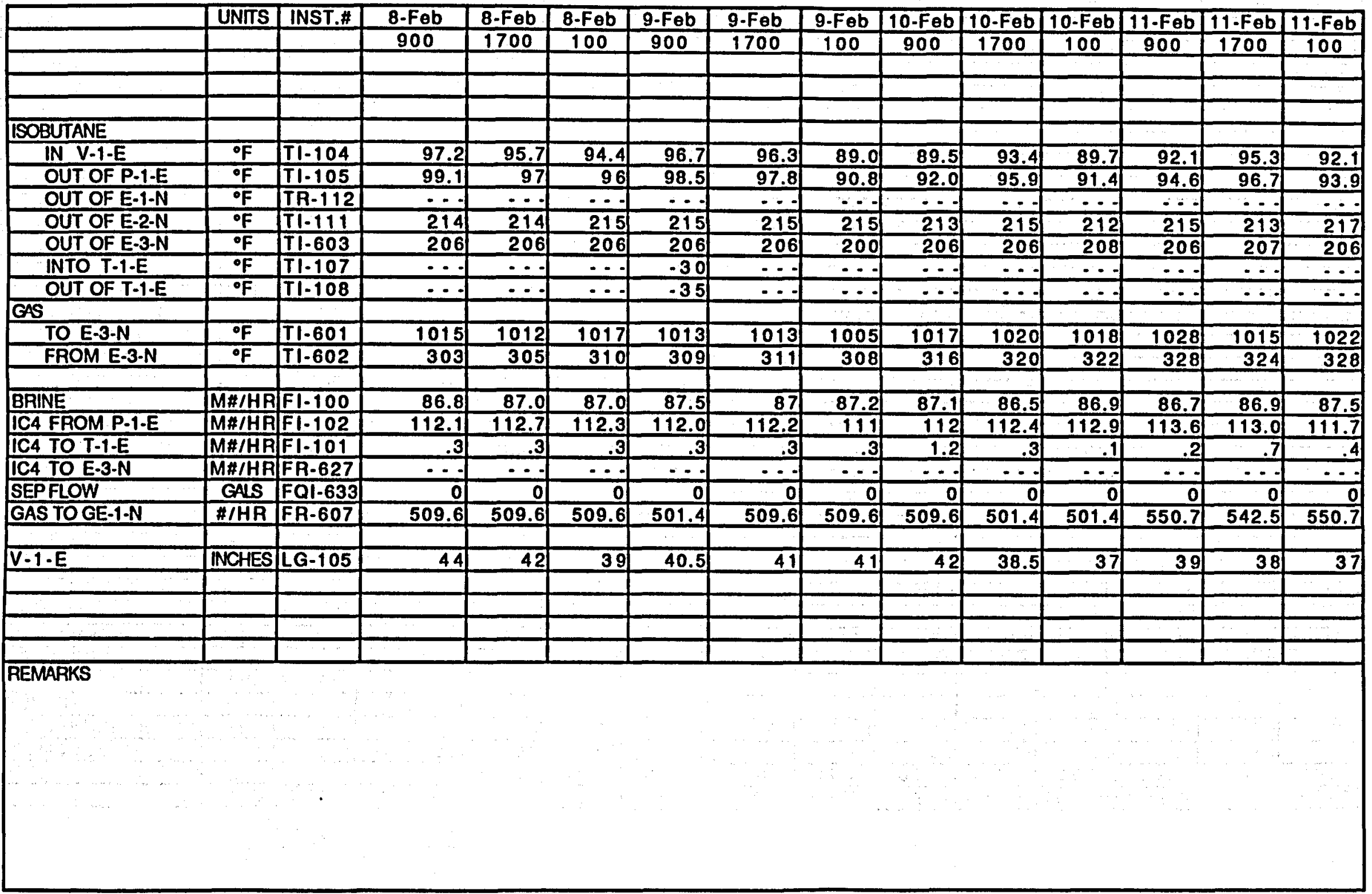


February 12-14 1990

\begin{tabular}{|c|c|c|c|c|c|c|c|c|c|c|}
\hline & INST.\# & 12-Fob & 12-Feb & 12-Feb & 13-Feb & 13-Fob & 13-Fob & $14-\mathrm{Feb}$ & 14-Feb & 14-Fob \\
\hline & & 900 & 1700 & 100 & 900 & 1700 & 100 & 900 & 1700 & 100 \\
\hline AMBIENT TEMP & & 64 & 66 & 58 & 71 & 68 & 64 & 70 & 71 & 68 \\
\hline WET BULB TEMP & & 61 & 60 & 56 & 66 & 65 & 64 & 68 & 69 & 67 \\
\hline \multicolumn{11}{|l|}{ POWER } \\
\hline T-1-N GROSS & $J 1-100$ & 1 & 1 & 1 & o) & 1 & 1 & 1 & 1 & 1 \\
\hline GE-1-N GROSS & & 340 & 340 & 340 & 340 & 340 & 340 & 340 & 335 & 340 \\
\hline GE-2-N GROSS & & 340 & 340 & 340 & 340 & 340 & 340 & 340 & 335 & 340 \\
\hline PAFASTIC & J1.101 & 172 & 202 & 202 & 200 & 206 & 187 & 186 & 205 & 205 \\
\hline NET SALE TOHLEP & & 500 & 480 & 480 & 480 & 470 & 480 & 500 & 480 & 460 \\
\hline NET SALE TOHL\&P & & 1605 & 1609 & 1613 & 1618 & 1622 & 1626 & 1630 & 1635 & 1639 \\
\hline \multicolumn{11}{|l|}{ 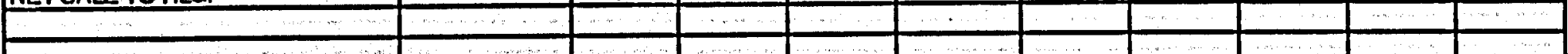 } \\
\hline & & & & & & & & & & \\
\hline BAINE & & & & & & & & & & \\
\hline TO E-2-N & $\mathrm{PI.606}$ & 505 & 500 & 500 & 505 & 500 & 500 & 505 & 501 & 500 \\
\hline TO E-1-N & $\mathrm{Pl} 1.611$ & 510 & 505 & 510 & 505 & 500 & 505 & 505 & 502 & 510 \\
\hline FROM E-1-N & $\mathrm{PI} 1.106$ & 497 & 493 & 500 & 494 & 491 & 500 & 496 & 493 & 500 \\
\hline \multicolumn{11}{|l|}{ ISOBUTANE } \\
\hline IN V-1.E & PG.130 & 59 & 64 & 62 & 65 & 58 & 60 & 59 & 59 & 58 \\
\hline OUT OF P.1-E & $\mathrm{PI}-105$ & 348 & 364 & 361 & 372 & 363 & 360 & 357 & 369 & 365 \\
\hline OUT OF E-I-N & P1.602 & 310 & 305 & 300 & 300 & 305 & 305 & 305 & 280 & 280 \\
\hline OUT OF E-2-N & $\overline{P G-114}$ & 270 & 265 & 260 & 265 & 265 & 265 & 265 & 265 & 270 \\
\hline OUT OF E-3-N & $P \mid-615$ & 275 & 275 & 275 & 270 & 275 & 275 & 270 & 272 & 275 \\
\hline INTO T-1-E & $P \mid-107$ & $\cdots$ & $\cdots$ & $\cdots$ & $\cdots$ & $\cdots$ & $\cdots$ & $\cdots$ & $\cdots$ & $\cdots$ \\
\hline OUT OF T-1-E & $P 1.108$ & $\cdots$ & $\cdots$ & $\cdots$ & $\cdots$ & $\cdots$ & $\cdots$ & $\cdots$ & $\cdots$ & $\cdots$ \\
\hline TO OR 102 & PG.104 & 330 & 330 & 340 & 335 & 330 & 330 & 340 & 332 & 330 \\
\hline FROM OA 102 & PG:105 & 140 & 150 & 150 & 145 & 145 & 140 & 145 & 142 & 140 \\
\hline \multicolumn{11}{|l|}{ 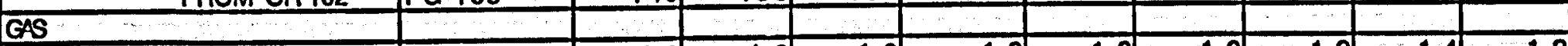 } \\
\hline TO E-3-N & $\mathrm{PI.614}$ & 1.0 & 1.0 & 1.0 & 1.2 & 1.2 & 1.2 & 1.2 & 1.4 & 1.2 \\
\hline \multirow{2}{*}{\multicolumn{11}{|c|}{ BRINE }} \\
\hline & & & & & & & & & & 208 \\
\hline TO E-2-N & $T I-101$ & 298 & 297 & 297 & $\frac{297}{\ldots}$ & $\frac{297}{207}$ & $\frac{297}{207}$ & $\frac{297}{207}$ & $\frac{298}{210}$ & $\frac{298}{207}$ \\
\hline TO E-1-N & $\frac{T A-113}{T R \cdot 103}$ & $\cdots$ & $\cdots$ & $\cdots$ & $\cdots$ & $\frac{204}{145}$ & $\frac{204}{144}$ & $\frac{204}{144}$ & $\frac{210}{144}$ & $\frac{201}{144}$ \\
\hline FROM E-1-N & TA. 102 & & & & & & & & & \\
\hline & & & & & & & & & & \\
\hline & & & & & & & & & & \\
\hline & & & & & & & & & & \\
\hline & & & & & & & & & & \\
\hline
\end{tabular}


Fobruary 12-14, 1990

\begin{tabular}{|c|c|c|c|c|c|c|c|c|c|c|}
\hline & UNITS & 12.Fob & 12-Feb & 12-Feb & 13-Fob & 13-Fob & 13-Feb & 14-Feb & 14-Feb & 14.Fob \\
\hline & & 900 & 1700 & 100 & 900 & 1700 & 100 & 900 & 1700 & 100 \\
\hline & & & & & & & & & & \\
\hline & & & & & & & & & & \\
\hline & & & & & & & & & & \\
\hline \multicolumn{11}{|l|}{ ISOBUTANE } \\
\hline IN $V-1-E$ & ० $\mathrm{F}$ & 94.0 & 99.2 & 97.0 & 100.0 & 91.3 & 95.0 & 95.3 & 94.2 & 93.6 \\
\hline OUT OF P-1-E & o $F$ & 96.5 & 101.5 & 99.1 & 102.5 & 91.0 & 96.8 & 97.1 & 96.1 & 95.4 \\
\hline OUT OF E-1-N & ${ }^{\circ} \mathrm{F}$ & $\because-$ & $\cdots$ & $\ldots$ & $\cdots$ & 199 & 199 & 199 & 199 & 199 \\
\hline OUT OF E-2-N & ${ }^{\circ} \mathrm{F}$ & 214 & 215 & 216 & 219 & 214 & 213 & 213 & 215 & 214 \\
\hline OUT OF E-3-N & ${ }^{\circ} \mathrm{F}$ & 207 & 206 & 206 & 205 & 206 & 206 & 206 & 207 & 206 \\
\hline INTO T-1-E & ०F & $\cdots$ & $\cdots$ & $\cdots$ & $\cdots$ & $\cdots$ & $\cdots$ & $\cdots$ & $\cdots$ & $\cdots$ \\
\hline OUT OF T-1-E & ${ }^{\circ} \mathrm{F}$ & $\cdots$ & $\cdots$ & $\cdots$ & $\cdots$ & $\cdots$ & $\cdots 1$ & $\cdots$ & $\cdots$ & $\cdots$ \\
\hline \multicolumn{11}{|l|}{ TOF } \\
\hline TO E-3-N & ${ }^{\circ} \mathrm{F}$ & 1018 & 1005 & 1013 & 1012 & 1009 & 1013 & 1012 & 1008 & 1012 \\
\hline FROM E-3.N & ${ }^{\circ} \mathrm{F}$ & 328 & 322 & 328 & 330 & 327 & 332 & 329 & 329 & 335 \\
\hline & & & & & & & & & & \\
\hline BRINE & M\#/HR & 86.6 & 86.8 & 86.9 & 86.7 & 86.9 & 87.1 & 086.9 & 87 & 87.1 \\
\hline IC4 FROM P-1-E & $\mathrm{M \# /HR}$ & 113.6 & 111.9 & 111.9 & 109.3 & 110.4 & 113.6 & 110.3 & 110 & 113.2 \\
\hline IC4 TO T-1-E & M\#/HR & .3 & .9 & .4 & .4 & .4 & .4 & .3 & .4 & .3 \\
\hline IC4 TO E-3-N & M\#/HR & $\because$ & $\cdots$ & $\cdots$ & $\cdots$ & 6.9 & 7.6 & 5.3 & 9.3 & 10.2 \\
\hline SEPFLOW & GAS & 0 & 0 & 0 & of & 0 & 0 & o & $\cdots$ & \\
\hline GAS TO GE-1-N & \#/HR & 550.7 & 542.5 & 542.5 & 534.3 & 545.4 & 539.8 & 544.9 & 539.8 & 545.9 \\
\hline & & & & & & & & & & \\
\hline$V .1-E$ & NCHES & 37 & 35 & 35 & 35 & 39 & 38 & 38.5 & 37 & 37 \\
\hline & & & & & & & & & & \\
\hline & & & & & & & & & & \\
\hline & & & & & & & & & & \\
\hline$=7$ & & & & & & & & & & \\
\hline
\end{tabular}


February 15-18, 1990

\begin{tabular}{|c|c|c|c|c|c|c|c|c|c|c|c|c|c|c|}
\hline & UNITS & INST.\# & 15. Fab & 15-Fob & 15.Fob & 16.Fab & 16-Fob & 16-Feb & 17.Feb & 17-Feb & 17.Fob & 18.Fob & 18.Fob & 18.Fob \\
\hline & & & 900 & 1700 & 100 & 900 & 1700 & 100 & 900 & 1700 & 100 & 900 & 1700 & 100 \\
\hline AMBIENT TEMP & of & & 69 & 76 & 70 & 57 & 63 & 54 & 51 & 54 & 52 & 51 & 53 & 54 \\
\hline WET BULB TEMP & ${ }^{\circ} \mathrm{F}$ & & 68 & 72 & 69 & 47 & 51 & 47 & 45 & 46 & 46 & 49 & 53 & 53 \\
\hline POWER & & & & & & & & & & & & & & \\
\hline T-1-N GROSS & $\overline{K W}$ & $\pi 1-100$ & 1 & 1 & 1 & 0 & 11 & 1 & 1 & 1 & 1 & 1 & 1 & 1 \\
\hline GE-1-NGROSS & $\mathrm{KW}$ & & 340 & 330 & 335 & 350 & 350 & 350 & 350 & 350 & 350 & 350 & 350 & 350 \\
\hline GE-2-N GROSS & $\mathrm{KW}$ & & 340 & 330 & 330 & 350 & 350 & 350 & 350 & 350 & 350 & 350 & 350 & 350 \\
\hline PARASTIC & $\overline{\mathrm{KW}}$ & $J 1-101$ & 194 & 194 & 204 & 168 & 168 & 158 & 168 & 171 & 181 & 181 & 158 & 168 \\
\hline NET SALE TOHL\&P & $\mathrm{KW}$ & & 470 & 470 & 460 & 510 & 530 & 500 & 515 & 510 & 470 & 490 & 520 & 500 \\
\hline NET SALE TO HL\&P & $\mathbf{P D G}$ & & 1643 & 1647 & 1651 & 1656 & 1660 & 1664 & 1669 & 1673 & 1678 & 1682 & 1687 & 1691 \\
\hline & $\mathrm{KW}-\mathrm{HA}$ & & & & $\cdots$ & 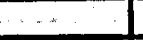 & 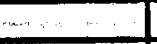 & - & 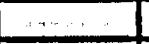 & & - & & $\cdots$ & \\
\hline & 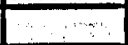 & & $\cdots$ & & 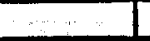 & & & & & & & & & \\
\hline BAINE & & & & $\ldots$ & & & & & & & & & & \\
\hline TO E-2.N & PSIG & PI-606 & 505 & 505 & 505 & 502 & 520 & 510 & 504 & 505 & 500 & 502 & 500 & 500 \\
\hline TO E.1.N & PSIG & $P \mid .611$ & 505 & 510 & 505 & 508 & 520 & 510 & 508 & 510 & 500 & 503 & 505 & 510 \\
\hline FAOM E-1-N & PSIG & PI.106 & 496 & 493 & 494 & 496 & 493 & 494 & 496 & 493 & 492 & 496 & 498 & 495 \\
\hline ISOBUTANE & & & & & & & & & & & & & & \\
\hline IN V.1.E & PSIG & PG-130 & 58 & 60 & 59 & 58 & 60 & 59 & 57 & 56 & 56 & 55 & 62 & 62 \\
\hline OUT OF P-1-E & PSIG & P1.105 & 362 & 358 & 354 & 342 & 342 & 354 & 349 & 346 & 352 & 354 & 357 & 358 \\
\hline OUT OF E-1-N & PSIG & $\overline{\mathrm{Pl} 1.602}$ & 280 & 285 & 285 & 278 & 280 & 230 & 274 & 285 & 280 & 280 & 280 & 285 \\
\hline OUT OF E-2-N & PSIG & PG-114 & 260 & 270 & 265 & 265 & 270 & 365 & 261 & 270 & 265 & 266 & 268 & 270 \\
\hline OUT OFE-3-N & PSIG & Pl.615 & 270 & 275 & 270 & 271 & 280 & 280 & 269 & 285 & 275 & 273 & 275 & 280 \\
\hline INTO T-1-E & PSIG & PI.107 & $\cdots$ & .95 & $\cdots$ & $\cdots$ & -95 & $\cdots$ & $\cdots$ & .95 & $\cdots$ & .95 & -95 & $\cdots$ \\
\hline OUT OF T-1-E & PSIG & PI-108 & $\cdots$ & .49 & $\cdots$ & $\cdots$ & -49 & $\cdots$ & $\cdots$ & 49 & $\cdots$ & -49 & -49 & $\cdots$ \\
\hline TO OA 102 & PSIG & PG-104 & 335 & 335 & 330 & 332 & 330 & 330 & 334 & 325 & 330 & 328 & 330 & 330 \\
\hline FROM OR 102 & PSIG & PG-105 & 145 & 145 & 145 & 148 & 150 & 150 & 148 & 140 & 140 & 141 & 140 & 140 \\
\hline GAS & & & & & & & & & & & & & & \\
\hline TOE-3-N & $\overline{\text { PSIG }}$ & PI-614 & 1.2 & 1.2 & 1.2 & 1.5 & 1.5 & 1.5 & 1.5 & 1.6 & 1.3 & 1.6 & 1.6 & 1.6 \\
\hline & & & & & & & & & & & & & & \\
\hline BAINE & & & & & & & & & & & & & & \\
\hline TO E-2-N & oF & $T I=101$ & 297 & 297 & 297 & 297 & 298 & 298 & 297 & 297 & 298 & 297 & 297 & 298 \\
\hline TO E-1-N & ०F & TR-113 & 207 & 208 & 206 & 207 & 208 & 206 & 206 & 206 & 206 & 206 & 206 & 211 \\
\hline FROM E-1-N & of & TR-102 & 144 & 145 & 140 & 144 & 147 & $\cdots$ & 143 & 143 & $\cdots$ & 113 & 143 & 147 \\
\hline & & & & & 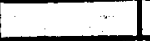 & & & & & & & & & \\
\hline & & & & & & & & & & & & & & \\
\hline & & & & & & & & & & & & & & \\
\hline & & & & & & & & & & & & & & \\
\hline & & & & & & & & & & & & & & \\
\hline
\end{tabular}




\section{GEOPRESSURED HYBRID POWER SYSTEM - MANAGEMENT REPORT DATA}

Fobruary 15-18, 1990

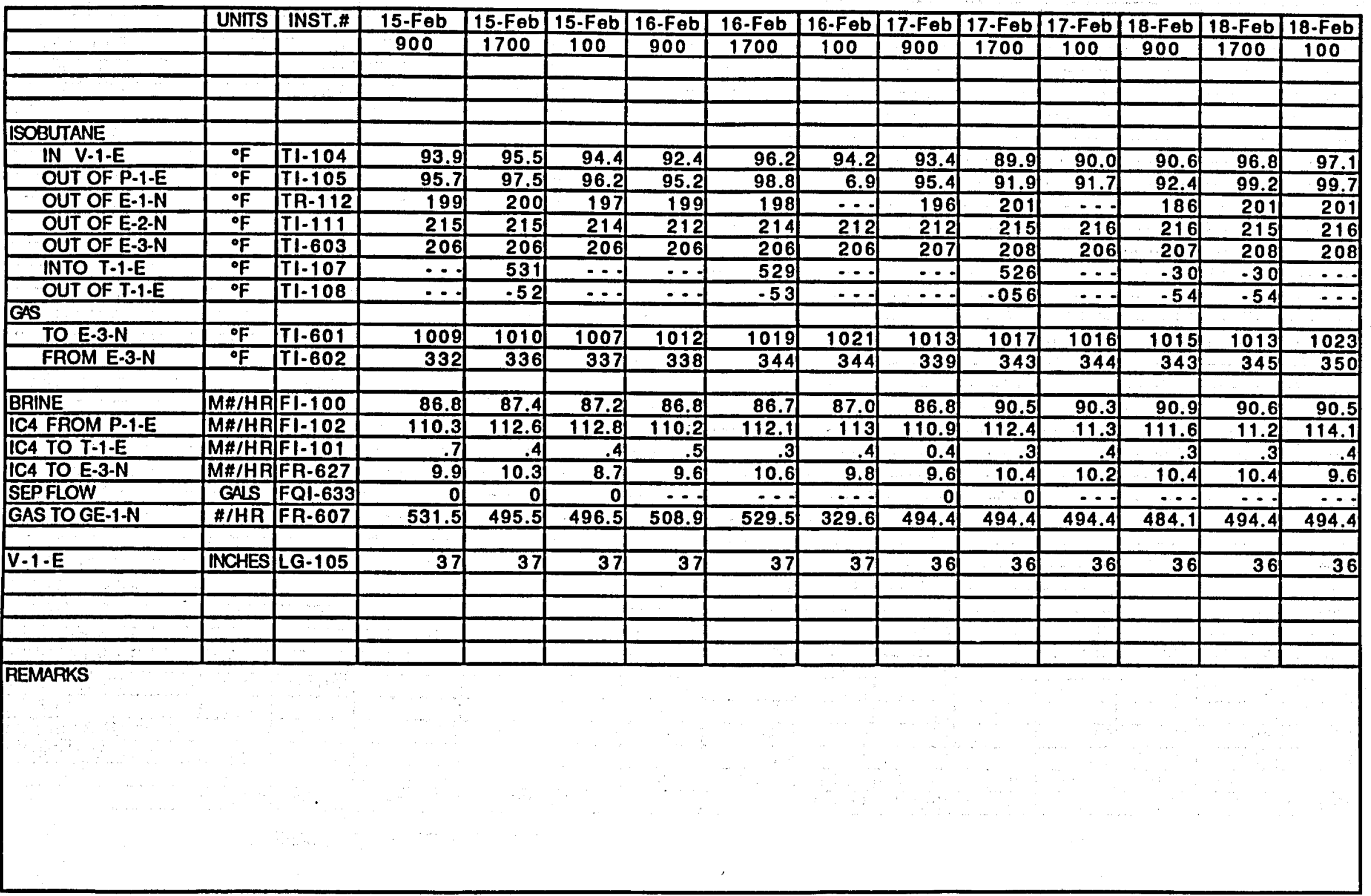


February 19-21, 1990

\begin{tabular}{|c|c|c|c|c|c|c|c|c|c|c|}
\hline & INST.\# & 19-Feb & 19.Feb & 19-Feb & 20-Feb & 20-Feb & 20-Feb & 21-Feb & 21-Feb & 21-Fob \\
\hline & & 900 & 1700 & 100 & 900 & 1700 & 100 & 900 & 1700 & 100 \\
\hline AMBIENT TEMP & & 56 & 62 & 53 & 54 & 59 & 60 & 64 & 54 & 50 \\
\hline WET BULB TEMP & & 54 & 57 & 50 & 54 & 55 & 50 & 64 & 53 & 49 \\
\hline \multicolumn{11}{|l|}{ POWEA } \\
\hline T-1-N GROSS & $J 1-100$ & 1 & 1 & 430 & 431 & 539 & 523 & 514 & 547 & 564 \\
\hline GE-1-N GROSS & & 350 & 350 & 350 & 350 & 350 & 350 & 350 & 350 & 30 \\
\hline GE-2-N GROSS & & 350 & 350 & 350 & 350 & 350 & 350 & 340 & 350 & 350 \\
\hline PARASIIC & $J 1.101$ & 168 & 180 & 237 & 238 & 242 & 253 & 251 & 263 & 245 \\
\hline NET SALE TOHL\&P & & 500 & 520 & 840 & 820 & 950 & 930 & 920 & 950 & 980 \\
\hline NET SALE TO HLQP & & 1696 & 1700 & 1707 & 1714 & 1722 & 1730 & 1739 & 1747 & 1755 \\
\hline \multirow{2}{*}{\multicolumn{11}{|c|}{+2}} \\
\hline & & & & & & & & & & \\
\hline \multicolumn{11}{|l|}{ BRINE } \\
\hline TOE-2-N & $\mathrm{PI} .606$ & 500 & 500 & 500 & 505 & 490 & 490 & 500 & 500 & 505 \\
\hline TOE-1-N & PI.611 & 505 & 505 & 500 & 505 & 490 & 490 & 490 & 495 & 495 \\
\hline FROM E-1-N & PI.106 & 496 & 494 & 485 & 496 & 484 & 482 & 480 & 485 & 484 \\
\hline \multicolumn{11}{|l|}{ ISOBUTANE } \\
\hline IN $V-1 \cdot E$ & PG-130 & 61 & 59 & 45 & 44 & 51 & 55 & 58 & 51 & 50 \\
\hline OUT OF P-1.E & PI.105 & 361 & 358 & 410 & 413 & 356 & 361 & 361 & 355 & 347 \\
\hline OUT OF E-1-N & P1.602 & 285 & 290 & 285 & 285 & 310 & 310 & 310 & 315 & 315 \\
\hline OUT OF E-2-N & PG-114 & 270 & 275 & 265 & 270 & 280 & 280 & 280 & 280 & 280 \\
\hline OUT OF E-3-N & PI.615 & 275 & 285 & 275 & 275 & 305 & 290 & 285 & 285 & 290 \\
\hline INTO T-1.E & $P \mid-107$ & $\cdots$ & $\cdots$ & $\cdots$ & 262 & 267 & 265 & 265 & 266 & 268 \\
\hline OUT OF T-1-E & $P 1.108$ & $\cdots$ & $\cdots$ & $\cdots$ & $\cdots$ & 56 & 58 & 60 & 55 & 52 \\
\hline TO OR 102 & PG-104 & 330 & 330 & 40 & 40 & 50 & 50 & 55 & 50 & 50 \\
\hline FROM OA 102 & PG-105 & 145 & 145 & 40 & 40 & 50 & 50 & 55 & 50 & 50 \\
\hline \multicolumn{11}{|l|}{ GAS } \\
\hline TO E-3-N & $\mathrm{PI}-614$ & 1.6 & 1.4 & 1.4 & 1.4 & 1.5 & 1.4 & 1.4 & 1.4 & 1.4 \\
\hline & & & & & & & $\therefore$ & & & \\
\hline \multicolumn{11}{|l|}{ BRINE } \\
\hline TO E-2-N & $T I-101$ & 298 & 298 & 297 & 297 & 297 & 297 & 297 & 297 & 298 \\
\hline TO E-1-N & TR-113 & 211 & 211 & 211 & 211 & 216 & 216 & 216 & 215 & 215 \\
\hline FROM E-1-N & TR-102 & 147 & 147 & $\ldots$ & 142 & 147 & 147 & 147 & 145 & 143 \\
\hline & & & & & & & & & & \\
\hline & & & & & & & & & & \\
\hline & & & & & & & & & & \\
\hline & & & & & & & & & & \\
\hline
\end{tabular}


Fobruary 19-21, 1990

\begin{tabular}{|c|c|c|c|c|c|c|c|c|c|c|}
\hline & UNITS & 19.Fob & 19-Fob & 19-Fab & $20-F_{\theta b}$ & $20-\mathrm{Feb}$ & $20 . \mathrm{Feb}$ & $21 . \mathrm{Feb}$ & $21 . \mathrm{Feb}^{-}$ & $21-\mathrm{Feb}$ \\
\hline & & 900 & 1700 & 100 & 900 & 1700 & 100 & 900 & 1700 & 100 \\
\hline & & & & & & & & & & \\
\hline & & & & & & & & & & \\
\hline & & & & & & & & & & \\
\hline \multicolumn{11}{|l|}{ ISOBUTANE } \\
\hline IN $V-1-E$ & o $F$ & 96.9 & 96.2 & 81.9 & 080.3 & 89.5 & 91.5 & 93.8 & 88.0 & 85.7 \\
\hline OUT OF P-1-E & ${ }^{\circ} \mathrm{F}$ & 99.7 & 98 & 83.2 & 81.6 & 90.7 & 92.9 & $\frac{90.0}{95.4}$ & 89.8 & .00 .6 \\
\hline OUT OF E-1-N & ${ }^{\circ} \mathrm{F}$ & 201 & 206 & 191 & 201 & 211 & 211 & 206 & 210 & 209 \\
\hline OUT OF E-2-N & ${ }^{\circ} \mathrm{F}$ & 215 & 221 & 216 & 217 & 220 & 221 & 220 & 220 & 218 \\
\hline OUT OF E-3-N & ${ }^{\circ} \mathrm{F}$ & 208 & 210 & 207 & 207 & 210 & 210 & 211 & 210 & 212 \\
\hline INTO T-1-E & of & $\cdots$ & $\cdots$ & $\cdots$ & $\cdots$ & 219 & 221 & 220 & 220 & 219 \\
\hline OUT OF T-1-E & of & $\cdots$ & $\cdots$ & 123 & 123 & 132 & 134 & 135 & 130 & 127 \\
\hline \multicolumn{11}{|l|}{ GAS } \\
\hline TO E-3-N & ${ }^{\circ} \mathrm{F}$ & 1021 & 1016 & 1007 & 1007 & 1008 & 1009 & 1001 & 1012 & 1018 \\
\hline FROM E-3-N & ${ }^{\circ} \mathrm{F}$ & 350 & 350 & 346 & 346 & 348 & 350 & 345 & 354 & 361 \\
\hline & & & & & & & & & & \\
\hline BAINE & M\#/HA & 90.3 & 90.8 & 100.3 & 100.1 & 142.5 & 142.3 & 143.3 & 141.8 & 142.5 \\
\hline IC4 FROM P-1.E & M\#/HA & 112.8 & 112 & 63.8 & 70.7 & 114.4 & 111.5 & 112.1 & 112.3 & 114.4 \\
\hline IC4 TO T-1.E & M\#/HA & .3 & .3 & 83.7 & 83.0 & 118.3 & 118.2 & 118.0 & 118.1 & 118.5 \\
\hline IC4 TO E-3.N & M\#/HA & 10.4 & 10.0 & 9.3 & 9.6 & 9.3 & 11.1 & 9.8 & 12.0 & 9.6 \\
\hline SEPFLOW & GALS & 0 & $\therefore 0$ & $\ldots$ & 0 & $\cdots$ & $\ldots$ & 0 & 0 & 0 \\
\hline \multirow[t]{2}{*}{ GAS TO GE-1-N } & \#/HA & 494.4 & 494.4 & 556.2 & 545.9 & 535.6 & 535.6 & 525.3 & 537.7 & 535.6 \\
\hline & & & & & & & & & & \\
\hline \multirow{4}{*}{$V-1-E$} & INCHES & 35 & 36 & 37 & 37 & 37 & 36.25 & 36.25 & 35.5 & 35 \\
\hline & & & & & & & & & & \\
\hline & & & & & & & & & & \\
\hline \multirow{5}{*}{\multicolumn{11}{|c|}{ REMARKS }} \\
\hline & & & & & & & & & & \\
\hline & & & & & & & & & & \\
\hline & & & & & & & & & & \\
\hline & & & & & & & & & & \\
\hline & & & & & & & & & & \\
\hline
\end{tabular}


February 22-25, 1990

\begin{tabular}{|c|c|c|c|c|c|c|c|c|c|c|c|c|c|c|}
\hline & UNTS & INST.\# & 22. $\mathrm{Fob}$ & 22-Fab & 22-Feb: & 23.Feb & 23-Feb & 23-Fab & 24-Feb & 24-Feb & 24.Fob & 25-Feb & 25.Feb & $25-\mathrm{Feb}$ \\
\hline & & & 900 & 1700 & 100 & 900 & 1700 & 100 & 900 & 1700 & 100 & 900 & 1700 & 100 \\
\hline AMBIENT TEMP & of & & 59 & 67 & 48 & 51 & 67 & 49 & 60 & 75 & 53 & 58 & 69 & 61 \\
\hline WET BULB TEMP & of & & 56 & 54 & 45 & 44 & 66 & 44 & 54 & 58 & 49 & 54 & 56 & 59 \\
\hline \multicolumn{15}{|l|}{ POWER } \\
\hline T.1.N GROSS & $\mathrm{KW}$ & $J 1.100$ & 542 & 537 & 579 & (0) & 0 & 1 & 1 & ol & 1 & 0 & 0 & 0 \\
\hline GE-1-NGROSS & KW & & 350 & 350 & 350 & 0 & 345 & 350 & 350 & 345 & 350 & 350 & 340 & 350 \\
\hline GE-2-N GROSS & KW & & 340 & 345 & 350 & 335 & 345 & 350 & 350 & 345 & 350 & 350 & 340 & 350 \\
\hline PARASTIC & $\mathrm{KW}$ & $\sqrt{1-101}$ & 254 & 253 & 257 & 178 & 157 & 157 & 156 & 183 & 171 & 170 & 170 & 170 \\
\hline NET SALE TOHL\&P & $\mathrm{KW}$ & & 950 & 945 & 950 & 200 & 525 & 520 & 540 & 505 & 520 & 520 & 515 & 520 \\
\hline \multirow[t]{2}{*}{ NET SALE TO HLEP } & FDG & & 1762 & 1769 & 1778 & 1784 & 1786 & 1790 & 1795 & 1799 & 1803 & 1808 & 1812 & 1818 \\
\hline & KW-HA & & & & & & & & & & & & & \\
\hline \multirow{2}{*}{\multicolumn{15}{|c|}{ BAINE }} \\
\hline & & & & & & & & & & & & & & \\
\hline TO E-2-N & PSIG & $\overline{\mathrm{PI} .606}$ & 500 & 507 & 500 & 505 & 508 & 505 & 510 & 506 & 500 & 500 & 505 & 500 \\
\hline TO E-1-N & PSIG & PI.611 & 490 & 497 & 500 & 515 & 511 & 515 & 510 & 509 & 510 & 510 & 505 & 505 \\
\hline FROM E-1-N & PSIG & $\mathrm{PI}-106$ & 486 & 485 & 486 & 506 & 494 & 497 & 505 & 496 & 494 & 500 & 494 & 497 \\
\hline \multicolumn{15}{|l|}{ ISOBUTANE } \\
\hline IN $\mathrm{V}-1-\mathrm{E}$ & PSIG & PG.130 & 52 & 52 & 46 & 54 & 611 & 58 & 62 & 57 & 54 & 56 & 57 & 59 \\
\hline OUT OF P.1-E & PSIG & $\mathrm{PI} 1.105$ & 351 & 354 & 340 & 399 & 352 & 355 & 354 & 355 & 356 & 356 & 368 & 366 \\
\hline OUT OF E-1-N & PSTG & PI.602 & 310 & 308 & 310 & 155 & 270 & 285 & 280 & 283 & 282 & 280 & 279 & 280 \\
\hline OUT OF E-2-N & PSIG & PG.114 & 280 & 276 & 280 & 150 & 259 & 270 & 270 & 272 & 270 & 270 & 267 & 270 \\
\hline OUT OF E-3-N & PSIG & $\mathrm{PI} 1.615$ & 285 & 283 & 285 & 155 & 266 & 280 & 275 & 277 & 282 & 280 & 272 & 275 \\
\hline INTO T-1-E & PSIG & $\mathrm{PI}: 107$ & 267 & 265 & 265 & 45 & 2 & 0 & 3 & 4 & 0 & 2 & 2 & 1 \\
\hline OUT OF T-1-E & $\overline{P S I G}$ & PI.108 & 55 & 55 & 47 & 47 & 11 & 11 & 14 & 12 & 11 & 14 & 11 & 11 \\
\hline TO OA 102 & PSIG & PG-104 & 50 & 50 & 50 & 380 & 338 & 330 & 335 & 330 & 330 & 330 & 332 & 330 \\
\hline FROM OR 102 & PSIG & PG-105 & 50 & 50 & 50 & 160 & 148 & 150 & 150 & 141 & 145 & 140 & 142 & 145 \\
\hline \multicolumn{15}{|l|}{$\begin{array}{l}\text { FROM OH } 102 \\
\text { GAS }\end{array}$} \\
\hline TO E-3-N & PSIG & PI.614 & 1.4 & 1.4 & 1.4 & 1.1 & 1.4 & 1.5 & 1.4 & .5 & .4 & .6 & .4 & 4 \\
\hline \multirow{2}{*}{\multicolumn{15}{|c|}{ BRINE }} \\
\hline & & & & & & & & & & & & & & \\
\hline TO E-2-N & of & $T 1.101$ & 298 & 297 & 298 & 297 & 297 & 296 & 297 & 298 & 298 & 298 & 297 & 297 \\
\hline TO E-1-N & o $F$ & TR-113 & 215 & 214 & 214 & 154 & 013 & 211 & 209 & 210 & 209 & 209 & 208 & 209 \\
\hline FAOM E-1-N & ${ }^{\circ} \mathrm{F}$ & TR-102 & 142 & 145 & 140 & 116 & 193 & 146 & 148 & 143 & 142 & 142 & 145 & 14,6 \\
\hline & & & & & & & & & & & & & & \\
\hline & & & & & & & & & & & & & & \\
\hline & & & & & & & & & & & & & & \\
\hline & & & & & & & & & & & & & & \\
\hline & & & & & & & & & & & & & & \\
\hline
\end{tabular}




\section{GEOPRESSURED HYBRID POWER SYSTEM - MANAGEMENT REPORT DATA}

February 22-25, 1990

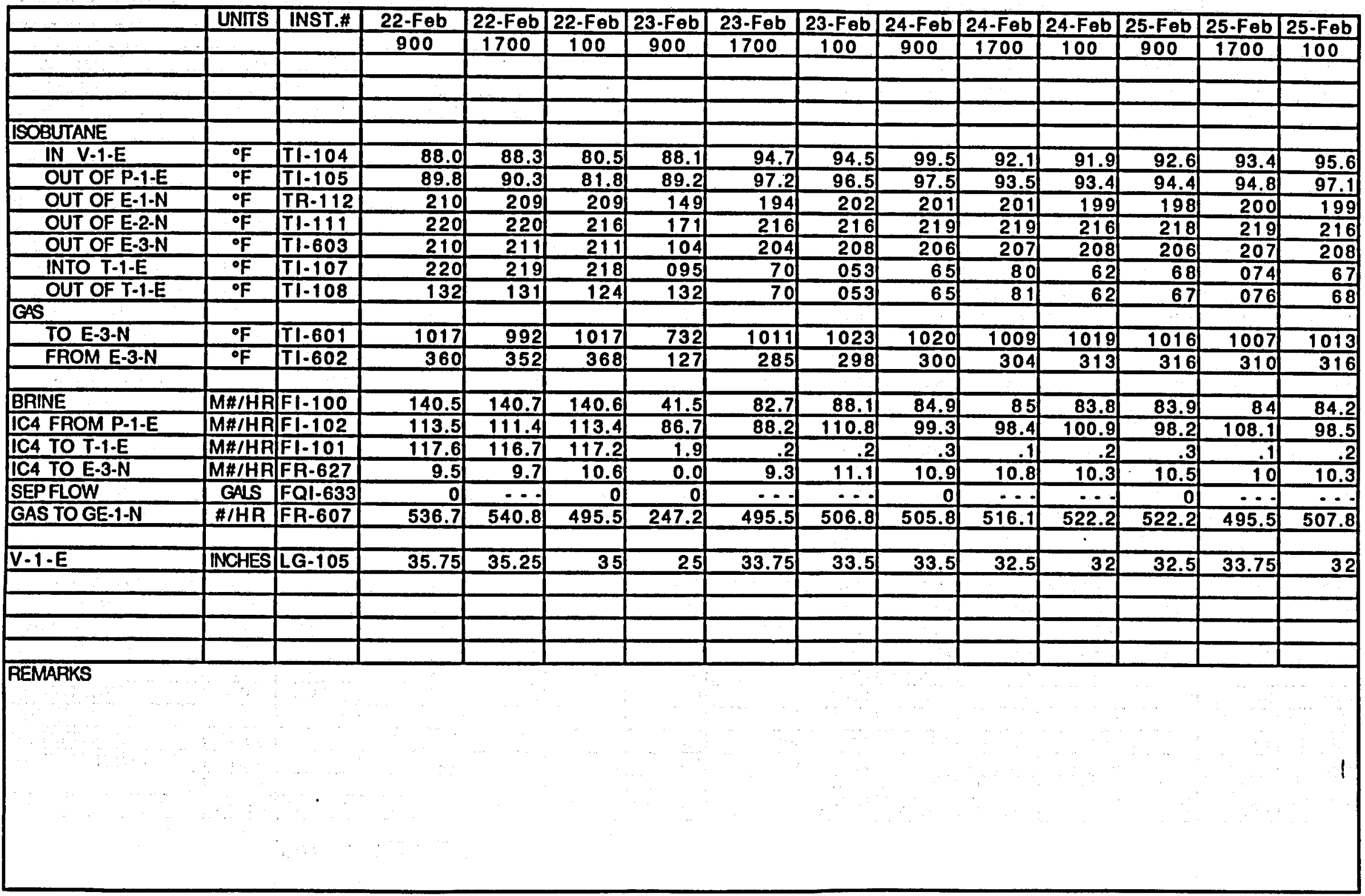


February 26-28, 1990

\begin{tabular}{|c|c|c|c|c|c|c|c|c|c|c|}
\hline & INST.\# & 26-Fob & 26-Feb/2 & $26-\mathrm{Feb}$ & 27-Feb & 27-Fob & 27.Feb & 28-Fob: & $28-F_{\theta b}$ & $28-\mathrm{Fab}$ \\
\hline & & 900 & 1700 & 100 & 900 & 1700 & 100 & \begin{tabular}{|l|}
900 \\
\end{tabular} & 1700 & 100 \\
\hline AMBIENT TEMP & & 66 & 67 & 60 & 68 & 67 & 64 & 70 & 68 & 53 \\
\hline WET BULB TEMP & & 60 & 62 & 58 & 64 & 63 & 63 & 67 & 66 & 51 \\
\hline POWER & & & & & & & & & & \\
\hline T-1-N GROSS & $J 1.100$ & 0 & 0 & 0 & of & of & 0 & 483 & 516 & 551 \\
\hline GE-1-N GROSS & & 350 & 350 & 350 & 350 & 350 & 350 & 350 & 340 & 350 \\
\hline GE-2-N GROSS & & 350 & 350 & 350 & 350 & 350 & 350 & 350 & 345 & 350 \\
\hline PAPASITIC & J1-1.01 & 169 & 180 & 181 & 169 & 180 & 190 & 240 & 252 & 256 \\
\hline NET SALE TO HL\&P & & 520 & 535 & 510 & 510 & 520 & 152 & 900 & 900 & 960 \\
\hline NET SALE TO HL\&P & & 1821 & 1826 & 1831 & 1835 & 1840 & 1844 & 1849 & 1857 & 1865 \\
\hline & & & & & 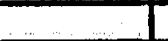 & & & & & \\
\hline & & & & & & & & & & \\
\hline BRINE & & & & & & & & & & \\
\hline TOE-2-N & P1.606 & 505 & 502 & 502 & 505 & 505 & 505 & 500 & 505 & 500 \\
\hline TO E-1-N & PI.611 & 505 & 503 & 505 & 505 & 505 & 505 & 500 & 490 & 490 \\
\hline FAOM E-1-N & $\mathrm{PI} \mid-106$ & 500 & 497 & 495 & 496 & 495 & 497 & 487 & 481 & 485 \\
\hline ISOBUTANE & & & & & & & & & & \\
\hline IN V-1.E & $\overline{P G-130}$ & 60 & 60 & 59 & 62 & 62 & 60 & 56 & 59 & 48 \\
\hline OUT OF P-1-E & PI.105 & 368 & 367 & 359 & 367 & 364 & 354 & 378 & 353 & 341 \\
\hline OUT OF E-1-N & $\mathrm{PI} .602$ & 280 & 279 & 278 & 275 & 280 & 260 & 285 & 315 & 310 \\
\hline OUT OF E-2-N & PG-114 & 265 & 266 & 265 & 265 & 265 & 270 & 265 & 280 & 280 \\
\hline OUT OF E-3-N & $P 1.615$ & 275 & 274 & 278 & 270 & 275 & 270 & 270 & 290 & 290 \\
\hline INTO T-1-E & $\overline{P I .107}$ & 2 & 2 & 1 & 3 & 2 & 44 & 276 & 269 & 264 \\
\hline OUT OF T-1-E & $\mathrm{PI} 1.108$ & 12 & 11 & 11 & 13 & 12 & 46 & 58 & 61 & 52 \\
\hline TO OA 102 & PG-104 & 330 & 335 & 335 & 335 & 335 & 330 & 55 & 60 & 50 \\
\hline FAOM OR 102 & PG-105 & 145 & 142 & 150 & 145 & 145 & 150 & 55 & 60 & 50 \\
\hline GAS & & & & & & & & & & \\
\hline TO E-3-N & PI.614 & .4 & .4 & .4 & .4 & 4 & .4 & .4 & .4 & .4 \\
\hline & 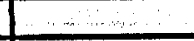 & & & 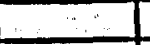 & & & & & & \\
\hline BAINE & & & & & & & & & & \\
\hline TO E-2-N & $T I-101$ & 297 & 297 & 297 & 297 & 297 & 297 & 297 & 297 & 297 \\
\hline TO E-1-N & TR-113 & 208 & 208 & 208 & 209 & 209 & 206 & 216 & 215 & 214 \\
\hline FAOM E-1-N & TR-102 & 145 & 146 & 146 & 148 & 148 & 145 & 148 & 149 & 145 \\
\hline & & & & & & & & & & \\
\hline & & & & & & & & & & \\
\hline & & & & & & & & & & \\
\hline & & & & & & & & & & \\
\hline & & & & & & & & & & \\
\hline
\end{tabular}


February 26-28, 1990

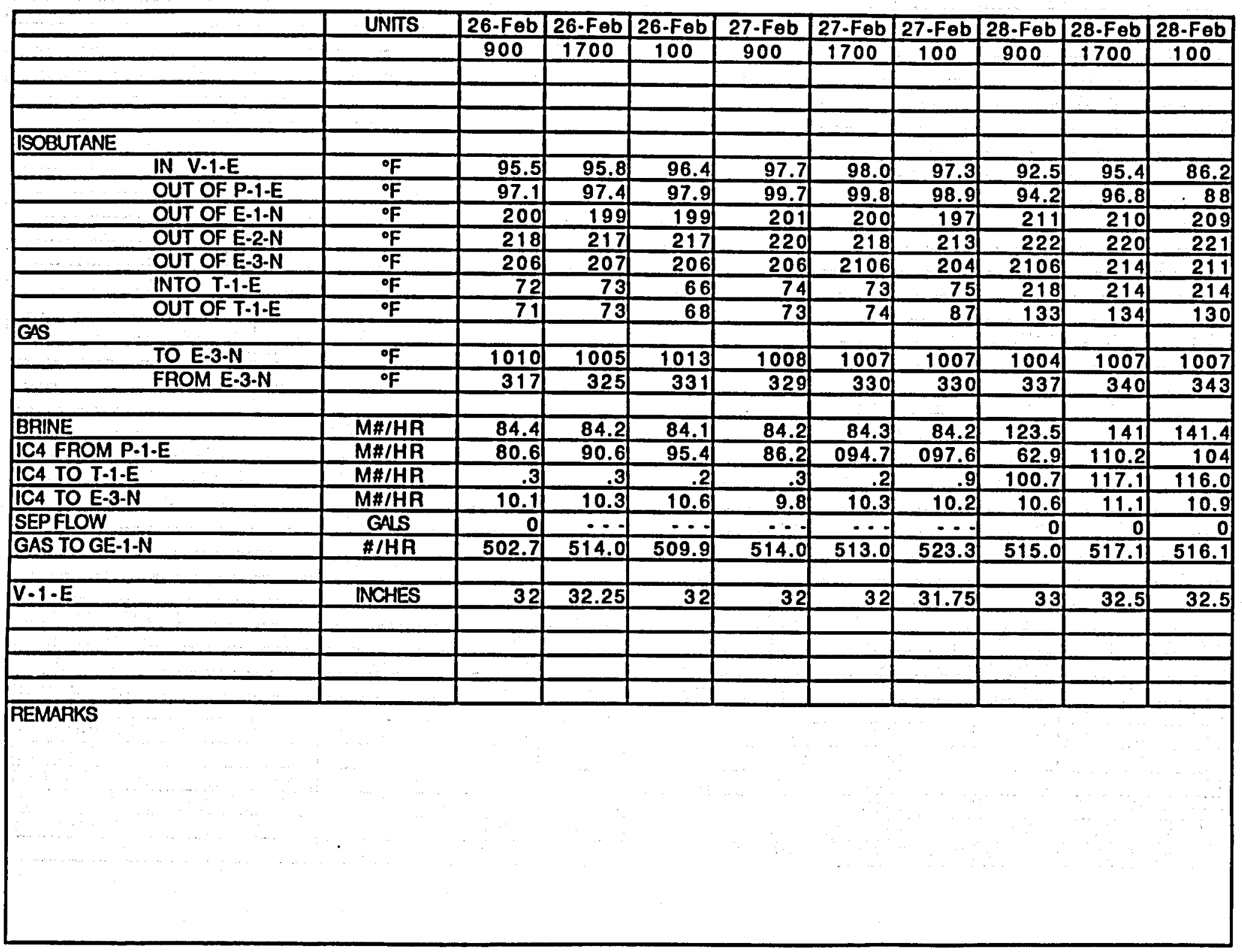


March 1.4, 1990

\begin{tabular}{|c|c|c|c|c|c|c|c|c|c|c|c|c|c|c|}
\hline & UNTS & INST.\# & 1-Mar & 1.Mar & 1.Mar & 2.Mar & 2-Mar & 2-Mar & 3-Mar & 3-Mar & 3-Mar & 4-Mar & 4-Mar & 4-Mar \\
\hline & & & 900 & 1700 & 100 & 900 & 1700 & 100 & 900 & 1700 & 100 & 900 & 1700 & 100 \\
\hline AMBIENT TEMP & ${ }^{\circ} \mathrm{F}$ & & 47 & 49 & 46 & 46 & 58 & 50 & 51 & 67 & 48 & 57 & 67 & 58 \\
\hline WET BULB TEMP & ${ }^{\circ} \mathrm{F}$ & & 47 & 49 & 44 & 44 & 51 & 47 & 47 & 58 & 48 & 53 & 59 & 54 \\
\hline \multicolumn{15}{|l|}{ POWEA } \\
\hline$T \cdot 1 \cdot N$ GROSS & KW & J1.100 & 566 & 555 & 571 & 569 & 559 & 569 & 561 & 0 & 0 & of & o & 520 \\
\hline GE-1-N GROSS & $\mathrm{KW}$ & & 350 & 350 & 350 & 350 & 350 & 350 & 350 & 340 & 350 & 350 & 345 & 345 \\
\hline GE-2-N GROSS & $\mathrm{KW}$ & & 350 & 350 & 350 & 350 & 350 & 350 & 350 & 340 & 350 & 350 & 345 & 340 \\
\hline PARASITIC & $\mathrm{KW}$ & $\mathrm{J1.101}$ & 246 & 245 & 246 & 29 & 244 & 244 & 245 & 6 & 16 & 16 & 36 & 253 \\
\hline NET SALE TOHL\&P & $\mathrm{KW}$ & & 980 & 950 & 960 & 950 & 975 & 960 & 945 & 630 & 640 & 650 & 625 & 920 \\
\hline \multirow{3}{*}{ NET SALE TO HL\&P } & $\mathbf{F D G}$ & & 1874 & 1882 & 1890 & 1899 & 1907 & 1915 & 1925 & 1930 & 1934 & 1941 & 1947 & 1953 \\
\hline & KW-HR & & & & 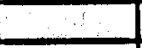 & & & & & & & & & \\
\hline \multirow{2}{*}{\multicolumn{15}{|c|}{ BRINE }} \\
\hline & & & & & & & & & & & & & & \\
\hline TO E-2-N & PSIG & PI.606 & 500 & 505 & 500 & 500 & 500 & 500 & 501 & 518 & 515 & 517 & 520 & 500 \\
\hline TO E-1-N & PSIG & PI-611 & 490 & 490 & 490 & 495 & 490 & 490 & 498 & 527 & 520 & 522 & 530 & 490 \\
\hline FROM E-1-N & PSIG & $\mathrm{PI} 1.106$ & 483 & 487 & 480 & 485 & 482 & 480 & 487 & 516 & 520 & 521 & 517 & 481 \\
\hline \multicolumn{15}{|l|}{ ISOBUTANE } \\
\hline IN V.1.E & PSIG & PG-130 & 48 & 47 & 46 & 46 & 50 & 50 & 47 & 31 & 24 & 22 & 28 & 52 \\
\hline OUT OF P-1-E & PSIG & $P 1-105$ & 313 & 353 & 349 & 351 & 349 & 352 & 357 & 30 & 18 & 16 & 25 & 350 \\
\hline OUT OF E-1-N & PSIG & $P 1.602$ & 310 & 285 & 290 & 290 & 295 & 290 & 284 & 35 & 25 & 25 & 30 & 285 \\
\hline OUT OFE-2-N & PSIG & PG-114 & 280 & 275 & 280 & 280 & 285 & 280 & 278 & 30 & 25 & 25 & 30 & 275 \\
\hline OUT OF E-3-N & PSIG & $\overline{P \mid-615}$ & 290 & 285 & 290 & 290 & 290 & 290 & 285 & 36 & 25 & 25 & 5 & 10 \\
\hline INTO T-1-E & PSIG & PI-107 & 266 & 264 & 267 & 266 & 270 & 271 & 264 & 29 & 21 & 22 & 29 & 266 \\
\hline OUT OF T-1-E & PSIG & PI-108 & 49 & 51 & 49 & 48 & 53 & 51 & 50 & 29 & 21 & 23 & 29 & 55 \\
\hline TO OA 102 & PSIG & PG-104 & 45 & 45 & 45 & 45 & 50 & 50 & 46 & 31 & 20 & 20 & 25 & 50 \\
\hline FROM OA 102 & PSIG & PG.105 & 45 & 45 & 45 & 45 & 50 & 50 & 46 & 31 & 20 & 20 & 25 & 50 \\
\hline \multicolumn{15}{|l|}{ GAS } \\
\hline TO E-3-N & PSIG. & PI-6.14 & 0.4 & 0.4 & 0.4 & 0.3 & 0.4 & 0.8 & 0.4 & .0 & .0 & .0 & .0 & .0 \\
\hline & & & & & & & & & 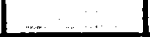 & & & & & \\
\hline \multicolumn{15}{|l|}{ BRINE } \\
\hline TOE-2-N & ${ }^{\circ} \mathrm{F}$ & $T 1-101$ & 297 & 297 & 297 & 297 & 297 & 298 & 298 & 271 & 267 & 267 & 265 & 298 \\
\hline TOE-1-N & ${ }^{\circ} \mathrm{F}$ & TR-113 & 215 & 214 & 215 & 215 & 214 & 215 & 92 & 77 & 66 & 60 & 64 & 213 \\
\hline FAOM E-1-N & ${ }^{\circ} \mathrm{F}$ & TR-102 & 145 & 143 & 142 & 142 & 144 & 143 & 91 & 75 & 68 & 68 & 65 & 149 \\
\hline & & & & & $\ldots$ & & & & & & & & & \\
\hline & & & & & & & & & & & & & & \\
\hline & & & & & & & & & & & & & & \\
\hline & & & & & & & & & & & & & & \\
\hline & & & & & & & & & & & & & & \\
\hline
\end{tabular}


GEOPRESSURED HYBRID POWER SYSTEM - MANAGEMENT REPORT DATA

March 1.4, 1990

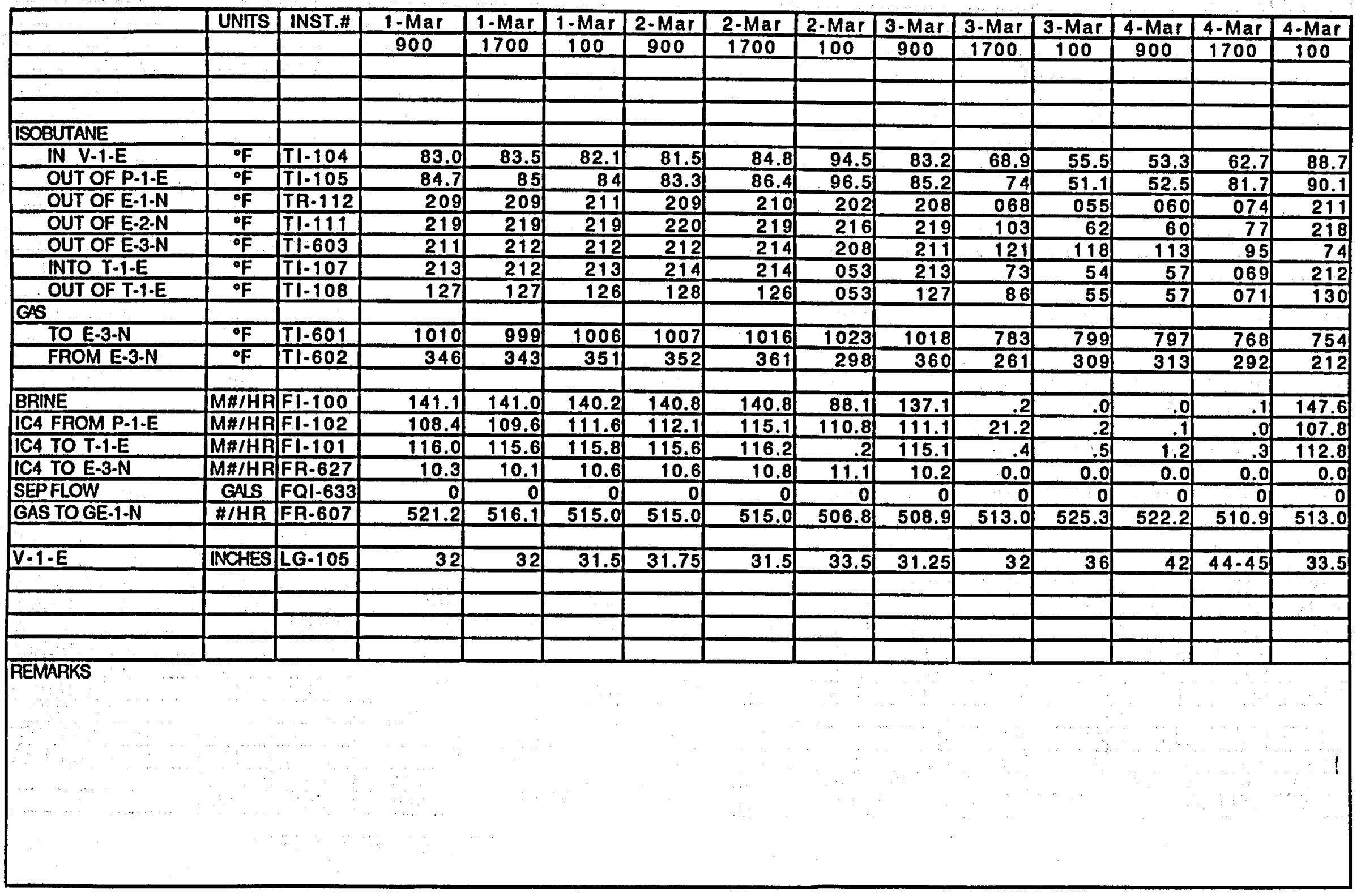


March 5-6, 1990

\begin{tabular}{|c|c|c|c|c|c|c|c|c|c|c|c|c|}
\hline & INST.\# & 5-Mar & 5-Mar & 5-Mar & 5-Mar & 5-Mar & 6-Mar & 6-MaP & 6-Mar & 6-Mar & 6-Mar & 6.Mar \\
\hline & & 900 & 1700 & 2100 & 100 & 500 & 900 & 1300 & 1700 & 2100 & 100 & 500 \\
\hline AMBIENT TEMP & & 66 & 67 & 62 & 63 & 63 & 69 & 73 & 69 & 68 & 68 & 67 \\
\hline WET BULB TEMP & & 60 & 62 & 60 & 60 & 61 & 66 & 69 & 66 & 66 & 66 & 66 \\
\hline \multicolumn{13}{|l|}{ POWEA } \\
\hline$T-1-N$ GROSS & $J 1.100$ & 527 & 515 & 520 & 510 & 519 & 0 & 491 & 0 & 499 & of & 0 \\
\hline GE-1-N GROSS & & 0 & 340 & 340 & 345 & 340 & 340 & 345 & 345 & 340 & 340 & 340 \\
\hline GE-2-N GROSS & & 0 & 340 & 340 & 340 & 340 & 345 & 0 & 340 & 345 & 340 & 320 \\
\hline PARASTIIC & J1.101 & 252 & 249 & 252 & 242 & 251 & 233 & 249 & 230 & 241 & 223 & 231 \\
\hline NET SALE TOHL\&P & & 305 & 910 & 920 & 900 & 900 & 640 & 595 & 460 & 890 & 460 & 430 \\
\hline \multirow{2}{*}{\multicolumn{13}{|c|}{ NET SALE TOHL\&P }} \\
\hline & & & & & & & & & & & & \\
\hline & & & & & & & & & & & & \\
\hline \multicolumn{13}{|l|}{ BRINE } \\
\hline TO E-2-N & $\mathrm{P} 1.606$ & 505 & 500 & 500 & 500 & 500 & 502 & 502 & 500 & 505 & 505 & 505 \\
\hline TOE-1.N & PI.611 & 490 & 490 & 490 & 490 & 490 & 502 & 489 & 500 & 490 & 505 & 505 \\
\hline FROM E-1-N & PI-106 & 481 & 483 & 481 & 480 & 480 & 493 & 481 & 492 & 481 & 494 & 494 \\
\hline \multicolumn{13}{|l|}{ SSOBUTANE } \\
\hline IN V.1.E & |PG-130 & 55 & 56 & 56 & 56 & 56 & 53 & 58 & 54 & 60 & 59 & 54 \\
\hline OUT OF P-1-E & $\mathrm{PI}-105$ & 353 & 353 & 344 & 342 & 334 & 287 & 376 & 435 & 358 & 430 & 430 \\
\hline OUT OF E-1-N & $\mathrm{PI} .602$ & 290 & 285 & 285 & 290 & 290 & 276 & 295 & 285 & 295 & 275 & 275 \\
\hline OUT OF E-2-N & PG-114 & 280 & 275 & 275 & 280 & 280 & 270 & 277 & 215 & 275 & 265 & 265 \\
\hline OUT OF E-3-N & $\mathrm{PI.615}$ & 0 & 280 & 280 & 290 & 290 & 276 & 282 & 285 & 285 & 275 & 270 \\
\hline INTO T-1-E & $\mathrm{PI}-107$ & 267 & 263 & 266 & 263 & 268 & 53 & 268 & 54 & 264 & 58 & 53 \\
\hline OUT OF T-1-E & $\mathrm{PI}-108$ & 57 & 58 & 59 & 59 & 60 & 53 & 61 & 54 & 63 & 59 & 54 \\
\hline TO. OR 102 & PG.104 & 52 & 55 & 55 & 55 & 55 & 49 & 57 & 50 & 60 & 55 & .500 \\
\hline FROM OR 102 & PG.105 & 52 & 55 & 50 & 55 & 55 & 0 & 57 & 50 & 60 & 55 & 500 \\
\hline \multicolumn{13}{|l|}{ 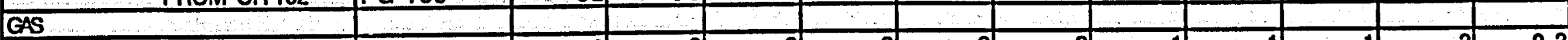 } \\
\hline TO E-3-N & $\mathrm{PI} 1.614$ & .0 & .9 & .3 & .3 & .3 & .2 & .1 & .1 & .1 & .2 & 0.2 \\
\hline \multirow{2}{*}{\multicolumn{13}{|c|}{ BRINE }} \\
\hline & & & & & & & & & & & & \\
\hline TO E-2.N & Tl.1.01 & 298 & 297 & 297 & 297 & 297 & 297 & 297 & $\frac{297}{210}$ & $\frac{297}{219}$ & $\frac{297}{200}$ & $\frac{297}{200}$ \\
\hline TO E-1-N & TR-113 & 215 & 213 & 213 & 214 & 214 & 210 & 213 & 210 & 213 & 209 & 200 \\
\hline FAOM E-1-N & TR.102 & 152 & 146 & 145 & 146 & 146 & 143 & 150 & 148 & 148 & 145 & 144 \\
\hline & & & & & & & & & & & & \\
\hline & & & & & & & & & & & & \\
\hline & & & & & & & & & & & & \\
\hline & & & & & & & & & & & & \\
\hline & & & & & & & & & & & & \\
\hline
\end{tabular}


March 5-6, 1990

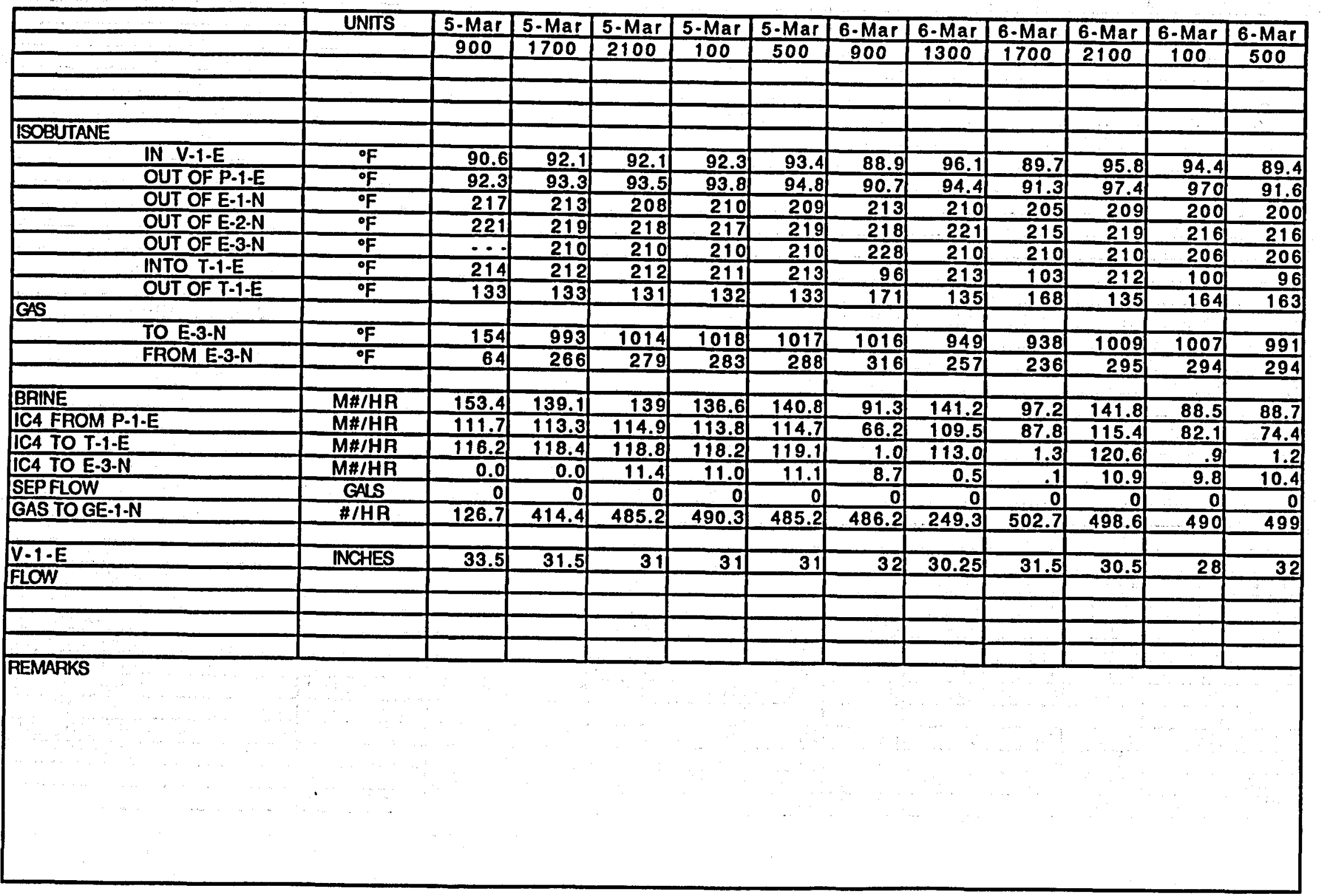


March 7,1990

\begin{tabular}{|c|c|c|c|c|c|c|c|c|c|c|c|c|}
\hline & INST.\# & 7.Mar & 7-Mar & 7-Mar & 7-Mar & 7-Mar & 7.Mar & & & & & \\
\hline & & 900 & 1300 & 1700 & 2100 & 100 & 500 & & & & & \\
\hline AMBIENT TEMP & & 70 & 75 & 71 & 69 & 69 & 69 & & & & & \\
\hline WET BULB TEMP & & 68 & 71 & 68 & 67 & 67 & 68 & & & & & \\
\hline POWER & & & & & & & & & & & & \\
\hline T-1-N GROSS & $\pi 1-100$ & 0 & 482 & 494 & 497 & 502 & 507 & & & & & \\
\hline GE-1-N GROSS & $\ldots \ldots$ & 340 & 340 & 345 & 345 & 345 & 340 & & & & & \\
\hline GE-2-N GROSS & & 340 & 340 & 345 & 350 & 350 & 340 & & & $\therefore$ & & \\
\hline PARASTIC & $J 1.101$ & 213 & 239 & 250 & 251 & 240 & 240 & & & & & \\
\hline NET SALE TOHL\&P & $\ldots$ & 470 & 850 & 900 & 900 & 900 & 900 & & & & & \\
\hline NET SALE TOHL\&P & & 1991 & 1994 & 1998 & 2002 & 2006 & 2010 & & & & & \\
\hline 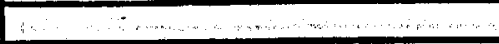 & $+\infty$ & & & $\therefore$ & $x^{2}$ & $\ldots$ & $\ldots$ & & & & & \\
\hline & & & & $\ldots$ & & $\ldots$ & & & & & & \\
\hline BRINE & & & & & & & & & & & & \\
\hline TO E-2-N & $\mathrm{P} 1-606$ & 505 & 500 & 502 & 502 & 500 & 500 & & & & $\ldots \ldots$ & \\
\hline TO E-1-N & PI-611 & 502 & 495 & 491 & 492 & 485 & 490 & & $-\infty$ & & 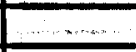 & \\
\hline FROM E-1-N & PI-106 & 493 & 482 & 481 & 482 & 480 & 482 & & & & & \\
\hline ISOBUTANE & & & & & & & & & & & & \\
\hline IN V.1-E & PG.130 & 56 & 58 & 60 & 60 & 60 & 60 & & & & & \\
\hline OUT OF P.1-E & $\mathrm{PI} 1.105$ & 442 & 359 & 354 & 355 & 356 & 352 & & & & - & \\
\hline OUT OF E-1-N & PI.602 & 275 & 297 & 298 & 295 & 300 & 300 & & & & & \\
\hline OUT OF E-2-N & PG.114 & 265 & 280 & 280 & 277 & 280 & 280 & & & & & \\
\hline OUT OFE-3-N & $P 1.615$ & 270 & 294 & 286 & 282 & 285 & 285 & & & & & \\
\hline INTO T-1.E & $P 1-107$ & 57 & 270 & 267 & 264 & 265 & 266 & & & & & \\
\hline OUT OF T-1-E & $P \mid-108$ & 60 & 63 & 63) & 63 & 63 & 64 & & & & & 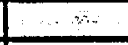 \\
\hline TO OR 102 & PG-104 & 50 & 55 & 60 & 60 & 60 & 60 & & & & & 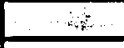 \\
\hline FROM OR 102 & PG.105 & 50 & 55 & 60 & 60 & 60 & 60 & & & & & \\
\hline GAS & & $\ldots 1$ & & & & $\ldots$. & & & & & & \\
\hline TO E-3-N & PI-614 & .1 & .4 & .2 & 3 & .2 & .2 & & & & & \\
\hline & & & & & & & & & & & & \\
\hline TO E.2.N & & & & & & & & & & & & \\
\hline $\begin{array}{l}\text { TOE-2.N } \\
\text { TO F-1.N }\end{array}$ & $T I-101$ & 297 & 297 & 297 & 297 & 298 & 298 & & & & & \\
\hline TO E-1.N & TR-113 & 208 & 214 & 216 & 213 & 214 & 214 & & & & & \\
\hline FROM E-1-N & TR.102 & 143 & 147 & 154 & 147 & 148 & 147 & & & & & \\
\hline & & & & & & & & & & & & \\
\hline & & & & & & & & & & & & \\
\hline & & & & & & & & & & & & \\
\hline & & & & & & & & & & & & \\
\hline
\end{tabular}


March 7,1990

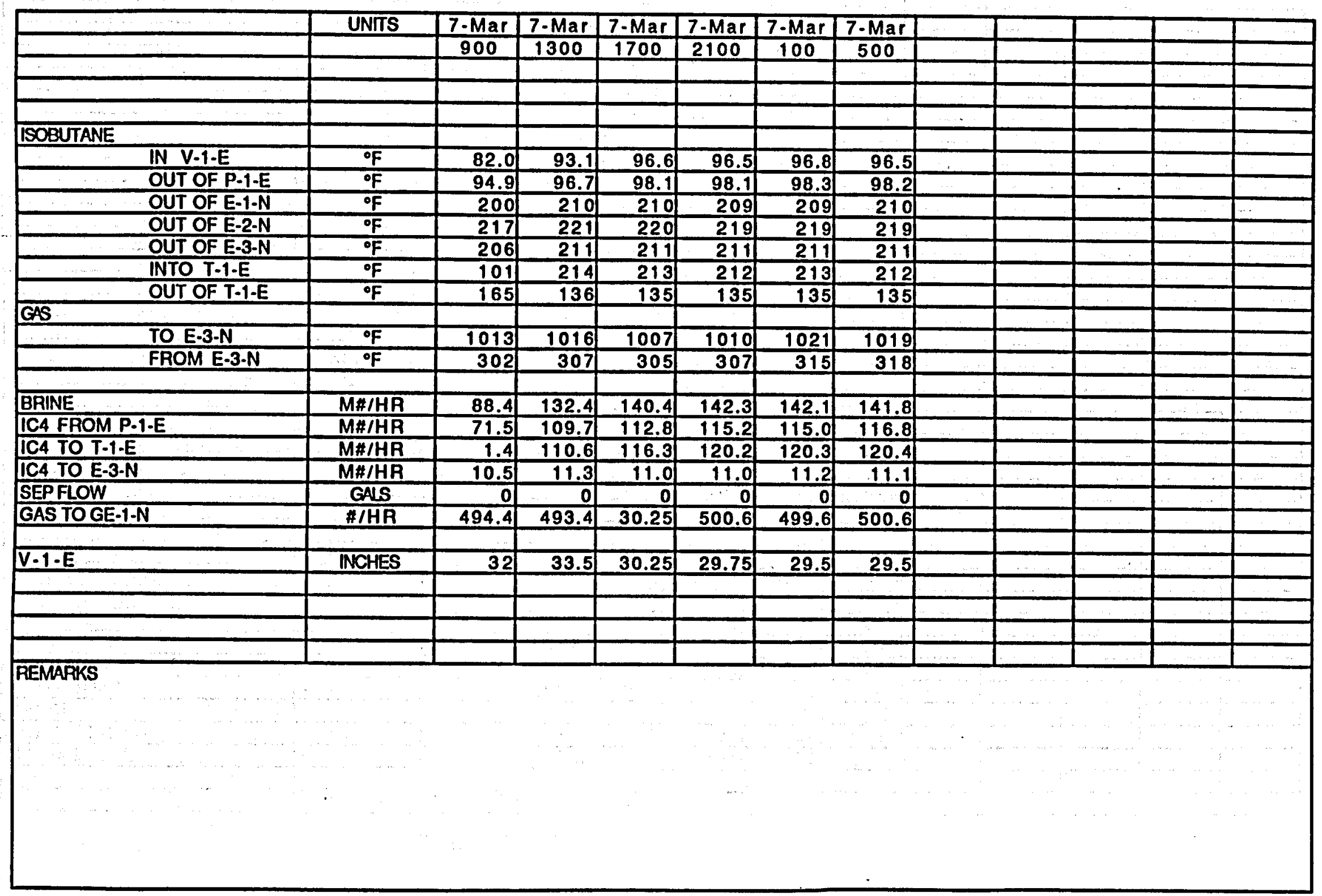


GEOPRESSURED HYBRID POWER SYSTEM - MANAGEMENT REPORT DATA

March 8.11, 1990

\begin{tabular}{|c|c|c|c|c|c|c|c|c|c|c|c|c|c|c|}
\hline & UNITS & INST.\# & 8-Mar & 8-Mar & 8-Mar & 9-Mar & 9-Mar & 9-Mar & 10-Mar 1 & 10-Mar & 10-Marl & 11-Mar & 11-Mar & 11-Mar \\
\hline & & & 900 & 1700 & 100 & 900 & 1700 & 100 & 900 & 1700 & 100 & 900 & 1700 & 100 \\
\hline AMBIENT TEMP & of & & 75 & 72 & 67 & 71 & 73 & 69 & 74 & 74 & 69 & 74 & 73 & 70 \\
\hline WET BULB TEMP & of & & 71 & 70 & 67 & 70 & 70 & 69 & 71 & 70 & 67 & 71 & 70 & 69 \\
\hline \multicolumn{15}{|l|}{ POWER } \\
\hline T.1-N GROSS & $\mathrm{KW}$ & $J 1.100$ & 500 & 491 & 501 & 499 & 498 & 501 & 501 & 493 & 500 & 501 & 491 & 499 \\
\hline GE-1-N GROSS & $\overline{K W}$ & & 340 & 340 & 340 & 340 & 340 & 340 & 340 & 340 & 340 & 340 & 340 & 340 \\
\hline GE-2-N GROSS & $\mathrm{KW}$ & & 340 & 330 & 340 & 340 & 340 & 335 & 340 & 340 & 340 & 340 & 340 & 340 \\
\hline PARASITIC & $\overline{\mathrm{KW}}$ & J1-101 & 240 & 240 & 252 & 248 & 239 & 252 & 250 & 239 & 250 & 240 & 241 & 240 \\
\hline NET SALE TOHL\&P & $\overline{K W}$ & & 890 & 880 & 900 & 890 & 880 & 890 & 900 & 880 & 895 & 900 & 900 & 890 \\
\hline \multirow[t]{3}{*}{ NET SALE TOHL\&P } & $\mathbf{A D G}$ & & 2014 & 2022 & 2029 & 2037 & 2045 & 2053 & 2061 & 2069 & 2076 & 2084 & 2092 & 2100 \\
\hline & KW-HA & & & & & & & & & & - & & & \\
\hline & & & & & & & & & & & & & & \\
\hline \multicolumn{15}{|l|}{ BAINE } \\
\hline$\frac{T O E-2-N}{T O}$ & PSIG & $P 1.606$ & 500 & 500 & 500 & 500 & 500 & 500 & 505 & 505 & 500 & 505 & 510 & 500 \\
\hline TO E-1-N & PSIG & $P \mid .611$ & 495 & 490 & 490 & 492 & 490 & 490 & 495 & 490 & 490 & 495 & 490 & 490 \\
\hline FROM E-1-N & PSIG & $P 1.106$ & 482 & 480 & 480 & 479 & 476 & 480 & 480 & 479 & 481 & 479 & 478 & 478 \\
\hline \multicolumn{15}{|l|}{ ISOBUTANE } \\
\hline IN V-1-E & PSIG & PG.130 & 60 & 60 & 60 & 58 & 63 & 61 & 60 & 62 & 60 & 61 & 62 & 61 \\
\hline OUT OF P-1.E & PSIG & $\mathrm{P} 1.105$ & 355 & 356 & 343 & 348 & 356 & 346 & 345 & 363 & 345 & 334 & 358 & 355 \\
\hline OUT OFE-1-N & PSIG & $\mathrm{PI}-602$ & 300 & 295 & 300 & 298 & 300 & 300 & 298 & 295 & 300 & 298 & 300 & 300 \\
\hline OUT OF E-2-N & PSIG & PG.114 & 280 & 280 & 275 & 280 & 280 & 280 & 280 & 280 & 280 & 280 & 280 & 280 \\
\hline OUT OFE-3-N & PSIG & $\mathrm{PI.615}$ & 290 & 275 & 290 & 290 & 285 & 290 & 292 & 285 & 290 & 295 & 285 & 290 \\
\hline INTO T-1-E & PSIG & PI.107 & 266 & 265 & 264 & 263 & 266 & 266 & 266 & 264 & 264 & 265 & 264 & 267 \\
\hline OUT OF T-1.E & PSIG & PI.108 & 65 & 64 & 63 & 64 & 65 & 64 & 65 & 65 & 64 & 65 & 66 & 65 \\
\hline TO OA 102 & PSIG & PG-104 & 60 & 60 & 60 & 60 & 60 & 60 & 60 & 60 & 60 & 60 & 60 & 60 \\
\hline FROM OA 102 & PSIG & PG:105 & 60 & 60 & 60 & 60 & 60 & 60 & 60 & 60 & 60 & 60 & 60 & 60 \\
\hline \multicolumn{15}{|l|}{ GS } \\
\hline TO E-3-N & PSIG & PI.614 & .4 & .4 & .1 & .4 & .3 & .2 & .4 & & $\because$ & .4 & .1 & .1 \\
\hline & & & & . & & & & & & & & & & \\
\hline \multicolumn{15}{|l|}{ BRINE } \\
\hline TO E-2-N & of & $T 1.101$ & 298 & 297 & 298 & 297 & 297 & 297 & 297 & 297 & 297 & 297 & 297 & 298 \\
\hline TO E-1-N & of & TR-113 & 214 & 214 & 214 & 214 & 214 & 214 & 214 & 214 & 213 & 213 & 214 & 215 \\
\hline FROM E-1-N & - $\mathrm{F}$ & TR-102 & 197 & 152 & 148 & 153 & 150 & 149 & 151 & 150 & 150 & 149 & 150 & 149 \\
\hline & & & & & & & & & & & & & & \\
\hline & & & & & & & & & & & & & & \\
\hline & & & & & & & & & & & & & & \\
\hline & & & & & & & & & & & & & & \\
\hline & & & & & & & & & & & & & & \\
\hline
\end{tabular}




\section{GEOPRESSURED HYBRID POWER SYSTEM - MANAGEMENT REPORT DATA}

March 8.11, 1990

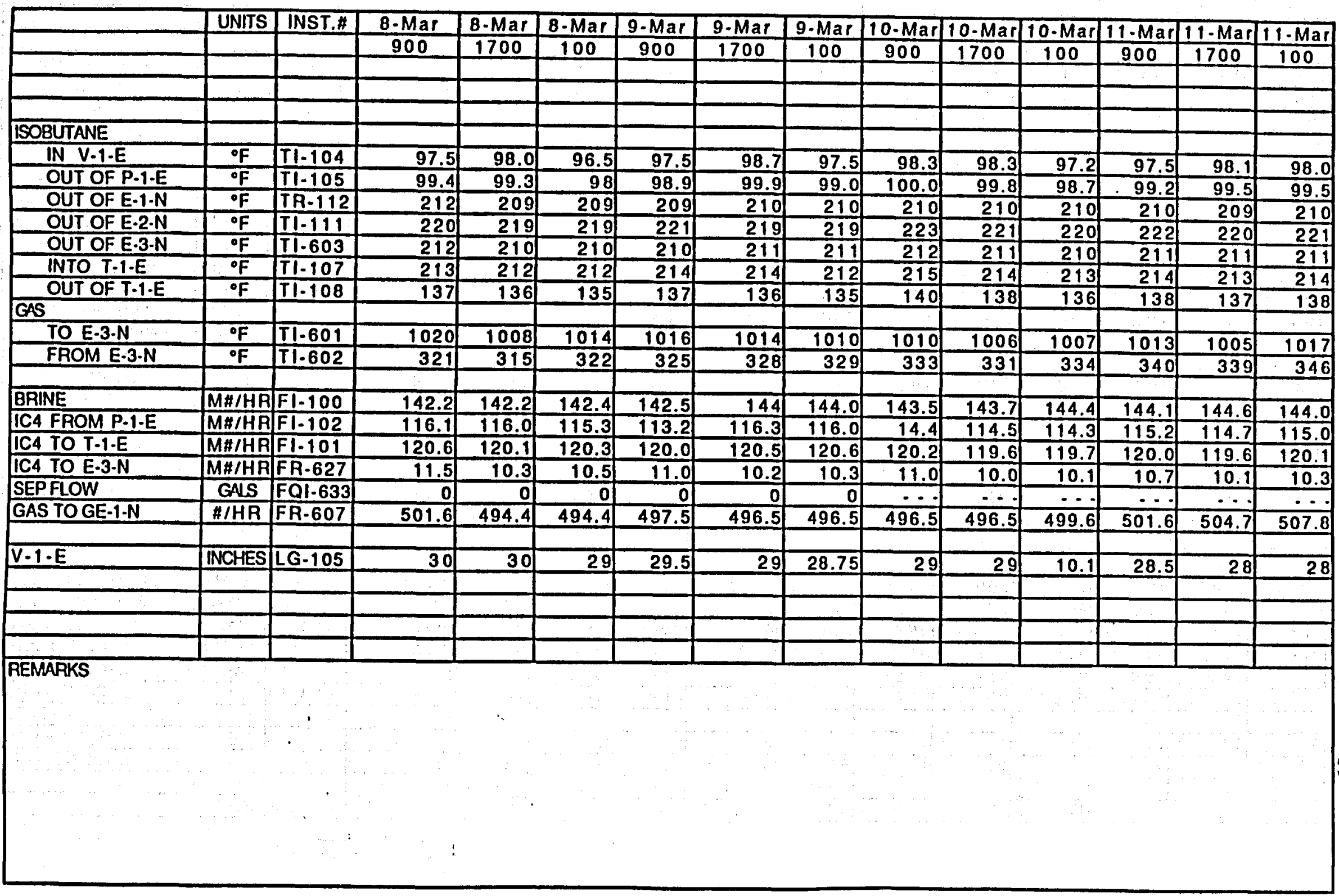


March 12-14, 1990

\begin{tabular}{|c|c|c|c|c|c|c|c|c|c|c|c|c|}
\hline & INST.\# & 12-Mar & 12-Mar & 12-Mar & 13-Mar & 13-Mar & 13-Mar & 14-Mar & 14-Mar & 14-Mar & & \\
\hline & & 900 & 1700 & 100 & 900 & 1700 & 100 & 900 & 1700 & 100 & & \\
\hline AMBIENT TEMP & & 75 & 67 & 68 & 74 & 71 & 70 & 70 & 73 & 59 & & \\
\hline WET BULB TEMP & & 71 & 67 & 67 & 71 & 69 & 69 & 68 & 71 & 56 & & \\
\hline \multicolumn{13}{|l|}{ POWER } \\
\hline T-1.N GROSS & $\mathrm{J} / 100$ & 488 & 501 & 482 & 1 & 0 & 0 & o & 0 & 0 & & \\
\hline GE-1.N GROSS & & 340 & 340 & 340 & 340 & 340 & 340 & 345 & 345 & 340 & & \\
\hline GE-2-NGROSS & & 340 & 340 & 0 & $\cdots$ & $\cdots$ & $\cdots$ & 0 & 140 & 330 & & \\
\hline PARASITIC & J1.101 & 239 & 241 & 251 & 236 & 181 & 191 & 191 & 193 & 192 & & \\
\hline NET SALE TOHL\&P & & 890 & 160 & 570 & 150 & 160 & 170 & 180 & 280 & 470 & & \\
\hline NET SALE TO HL\&P & & 2108 & 2115 & 2120 & 2125 & 2126 & 2128 & 2129 & 2131 & 2135 & & \\
\hline & & & & & & & & & & & & \\
\hline \multirow{2}{*}{\multicolumn{8}{|c|}{ BRINE }} & & & & & \\
\hline & & & & 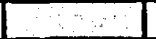 & & & & & & & & \\
\hline TO E.2.N & PI.606 & 500 & 500 & 500 & 509 & 505 & 500 & 502 & 510 & 500 & & \\
\hline TO E-1.N & PI.611 & 490 & 490 & 490 & 505 & 500 & 500 & 502 & 510 & 505 & & \\
\hline FROM E-1.N & PI.106 & 477 & 478 & 480 & 479 & 492 & 494 & 493 & 495 & 493 & & \\
\hline \multicolumn{13}{|l|}{ ISOBUTANE } \\
\hline IN $V-1-E$ & PG-130 & 60 & 60 & 59 & 58 & 60 & 61 & 60 & 61 & 56 & & \\
\hline OUT OF P.1-E & P/.105 & 349 & 349 & 361 & 421 & 359 & 370 & 362 & 360 & 350 & & \\
\hline OUT OF E-1.N & P).602 & 298 & 295 & 300 & 280 & 275 & 275 & 275 & 280 & 290 & & \\
\hline OUT OF E-2-N & PG-114 & 280 & 280 & 280 & 270 & 265 & 265 & 266 & 265 & 275 & & \\
\hline OUT OF E-3-N & P/.615 & 290 & 290 & 285 & 275 & 270 & 270 & 269 & 272 & 285 & & \\
\hline INTO T.1.E & PI.107 & 265 & 266 & 264 & 55 & 1 & 1 & 1 & 1 & 0 & & \\
\hline OUT OF T-1-E & PI-108 & 65 & 63 & 61 & 56 & 8 & 9 & 9 & 9 & 8 & & \\
\hline TO OR 102 & PG-104 & 60 & 60 & 60 & 55 & 140 & 145 & 339 & 330 & 320 & & \\
\hline FAOM OR 102 & PG-105 & 60 & 60 & 60 & 55 & 330 & 330 & 148 & 140 & 140 & & \\
\hline \multicolumn{13}{|l|}{ ginivitive } \\
\hline TO E-3.N & $\mathrm{P} \mid-614$ & .4 & .1 & .1 & .4 & .1 & .1 & .1 & .1 & .2 & & \\
\hline & & & & & & & & $\therefore$ & & & & \\
\hline \multicolumn{13}{|l|}{ BRINE } \\
\hline TO E.2.N & $T 1-101$ & 297 & 296 & 297 & 297 & 297 & 297 & 297 & 297 & 298 & & \\
\hline TO E-1.N & TR.113 & 214 & 215 & 213 & 223 & 207 & 208 & 208 & 209 & 212 & & \\
\hline FROM E-1-N & TR-102 & 151 & 140 & 146 & 156 & 148 & 149 & 148 & 164 & 145 & & \\
\hline & & & & & & & & & & $\therefore$ & & \\
\hline & & & & & & & & & & & & \\
\hline & & & & & & & & & & & & \\
\hline & & & & & & & & & & & & \\
\hline & & & & & & & & & & & & \\
\hline
\end{tabular}


March 12.14, 1990

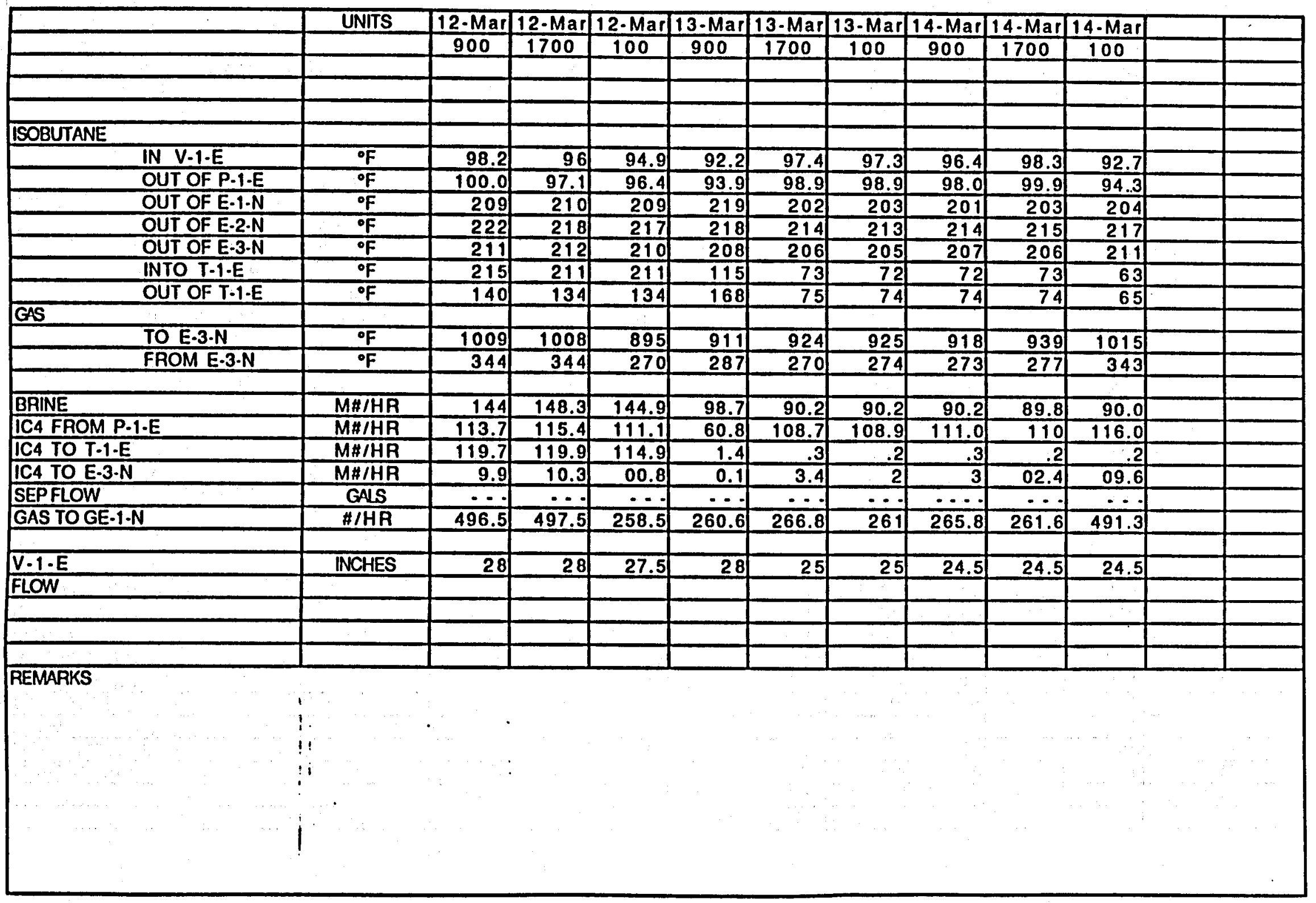


March $15.18,1990$

\begin{tabular}{|c|c|c|c|c|c|c|c|c|c|c|c|c|c|c|}
\hline & UNTS & INST.\# & 15-Mar & 15-Mar & 15-Mar & 16-Mar & 16-Mar & 16-Mar & 17-Mar & 17-Mar & 17-Mar & 18-Mar & 18-Mar & 18-Mar \\
\hline & & & 900 & 1700 & 100 & 900 & 1700 & 100 & 900 & \begin{tabular}{|l|l|}
1700 \\
\end{tabular} & 100 & 900 & 1700 & 100 \\
\hline AMBIENT TEMP & ${ }^{\circ} \mathrm{F}$ & & 52 & 62 & 53 & 57 & 64 & 54 & 60 & 72 & 55 & 69 & 71 & 58 \\
\hline WET BULB TEMP & oF & & 51 & 57 & 71 & 55 & 58 & 52 & 54 & 57 & 53 & 65 & 61 & 55 \\
\hline \multicolumn{15}{|l|}{ POWER } \\
\hline T-1-N GROSS & $\mathrm{KW}$ & $J 1-100$ & 0 & of & 0 & 0 & of & of & of & of & 0 & of & 0 & 0 \\
\hline GE-1-N GROSS & KW & & 340 & 345 & 345 & 350 & 350 & 350 & 350 & 340 & 340 & 345 & 345 & 345 \\
\hline GE-2-N GROSS & KW & & 330 & 345 & 345 & 350 & 350 & 350 & 350 & 340 & 340 & 345 & 345 & 345 \\
\hline PARASTIC & $\mathrm{KW}$ & J1-101 & 170 & 179 & 180 & 169 & 180 & 1770 & 169 & 178 & 179 & 168 & 169 & 170 \\
\hline NET SALE TOHL\&P & KW & & 480 & 515 & 520 & 505 & 520 & 500 & 520 & 500 & 500 & 515 & 520 & 510 \\
\hline \multirow{3}{*}{ NET SALE TOHL\&P } & FDG & & 2138 & 2143 & 2146 & 2152 & 2157 & 2161 & 21.66 & 2170 & 2174 & 2179 & 2183 & 2188 \\
\hline & KW.HA & & & & & & & & & & & $\ldots$ & & \\
\hline & & & & & & & & & & & & & & \\
\hline \multicolumn{15}{|l|}{ BAINE } \\
\hline TO E-2.N & $\overline{\mathbf{P S I G}}$ & $\mathrm{P} 1.606$ & 500 & 501 & 500 & 501 & 502 & 500 & 508 & 505 & 500 & 503 & 505 & 500 \\
\hline TO E-1-N & PSIG & P1.611 & 505 & 501 & 505 & 502 & 502 & 510 & 511 & 505 & 500 & 509 & 505 & 500 \\
\hline FROM E-1-N & $\overline{P S \mid G}$ & $P \mid-106$ & 494 & 491 & 492 & 493 & 490 & 496 & 496 & 492 & 494 & 493 & 493 & 494 \\
\hline \multicolumn{15}{|l|}{ ISOBUTANE } \\
\hline IN V-1-E & PSIG & PG.130 & 57 & 60 & 58 & 58 & 58 & 58 & 58 & 58 & 58 & 61 & 58 & 58 \\
\hline OUT OF P.1-E & PSIG & $P \mid-105$ & 348 & 341 & 345 & 344 & 346 & 341 & 351 & 356 & 360 & 358 & 365 & 354 \\
\hline OUT OF E-1-N & PSIG & $P 1.602$ & 285 & 286 & 285 & 286 & 285 & 290 & 286 & 290 & 285 & 287 & 286 & 285 \\
\hline OUT OF E-2-N & PSIG & PG.114 & 275 & 275 & 275 & 276 & 275 & 280 & 277 & 280 & 280 & 279 & 280 & 275 \\
\hline OUT OF E-3-N & $\overline{\text { PSIG }}$ & $P 1.615$ & 285 & 283 & 285 & 283 & 285 & 290 & 283 & 290 & 285 & 284 & 290 & 285 \\
\hline INTO T-T-E & $\overline{P S \mid G}$ & $\mathrm{Pl}-107$ & 0 & 1 & 0 & 0 & 1 & 0 & 2 & 1 & 0 & 1 & 1 & 0 \\
\hline OUT OF T.1-E & PSIG & Pl.108 & 8 & 8 & 8 & 8 & 7 & 8 & 11 & 7 & 8 & 10 & 7 & \\
\hline TO OR 102 & PSIG & $\overline{P G-104}$ & 330 & 330 & 330 & 328 & 330 & 320 & 330 & 330 & 330 & 330 & 325 & $\begin{array}{r}325 \\
\end{array}$ \\
\hline FROM OR 102 & PSIG & PG-105 & 145 & 144 & 145 & 148 & 140 & 145 & 148 & 150 & 150 & 147 & 145 & 145 \\
\hline \multicolumn{15}{|l|}{ GAS } \\
\hline TO E-3-N & PSIG & $\mathrm{P} \mid-614$ & .2 & .2 & .2 & .2 & .2 & .2 & .2 & .2 & .2 & .2 & .2 & .2 \\
\hline & $n+\infty$ & & & & & & & & & & & & & \\
\hline \multicolumn{15}{|l|}{ BRINE } \\
\hline TO E-2-N & ०F & $T 1-101$ & 298 & 297 & 298 & 298 & 297 & 298 & 298 & 298 & 298 & 298 & 297 & 298 \\
\hline TO E-1-N & ०F & TR.113 & 212 & 212 & 211 & 212 & 211 & 211 & 212 & 212 & 212 & 212 & 212 & 212 \\
\hline FROM E-1-N & ०F & TR-102 & 145 & 145 & 144 & 145 & 148 & 146 & 179 & 147 & 178 & 212 & 148 & 14.7 \\
\hline & & & & & & & & & & & & & & \\
\hline & & & & & & & & & & & & & & \\
\hline & & & & & & & & & & & & & & \\
\hline & & & & & & & & & & & & & & \\
\hline & & & & & & & & & & & & & & \\
\hline
\end{tabular}


GEOPRESSURED HYBRID POWER SYSTEM - MANAGEMENT REPORT DATA

March 15-18, 1990

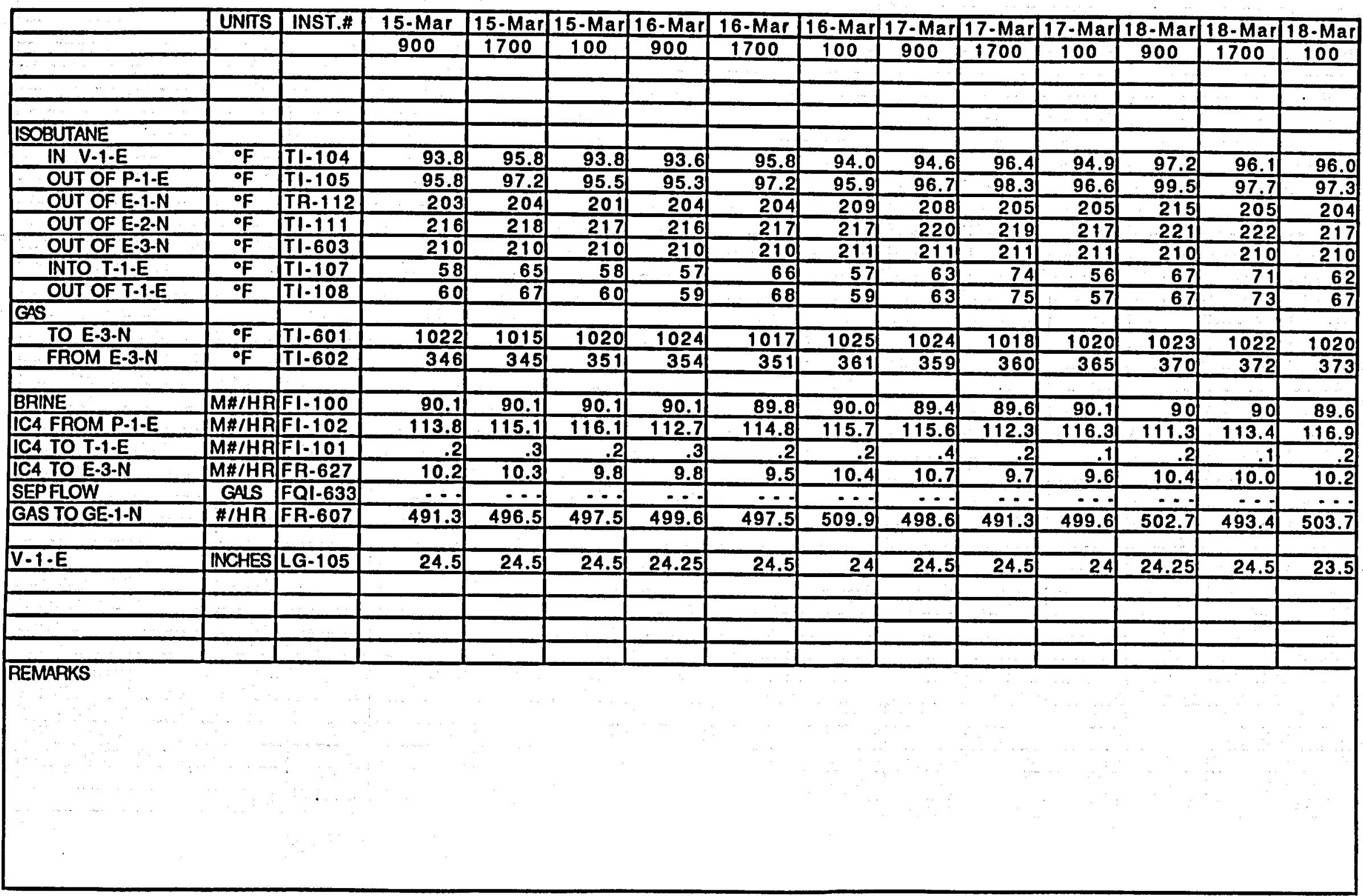


March 19-21, 1990

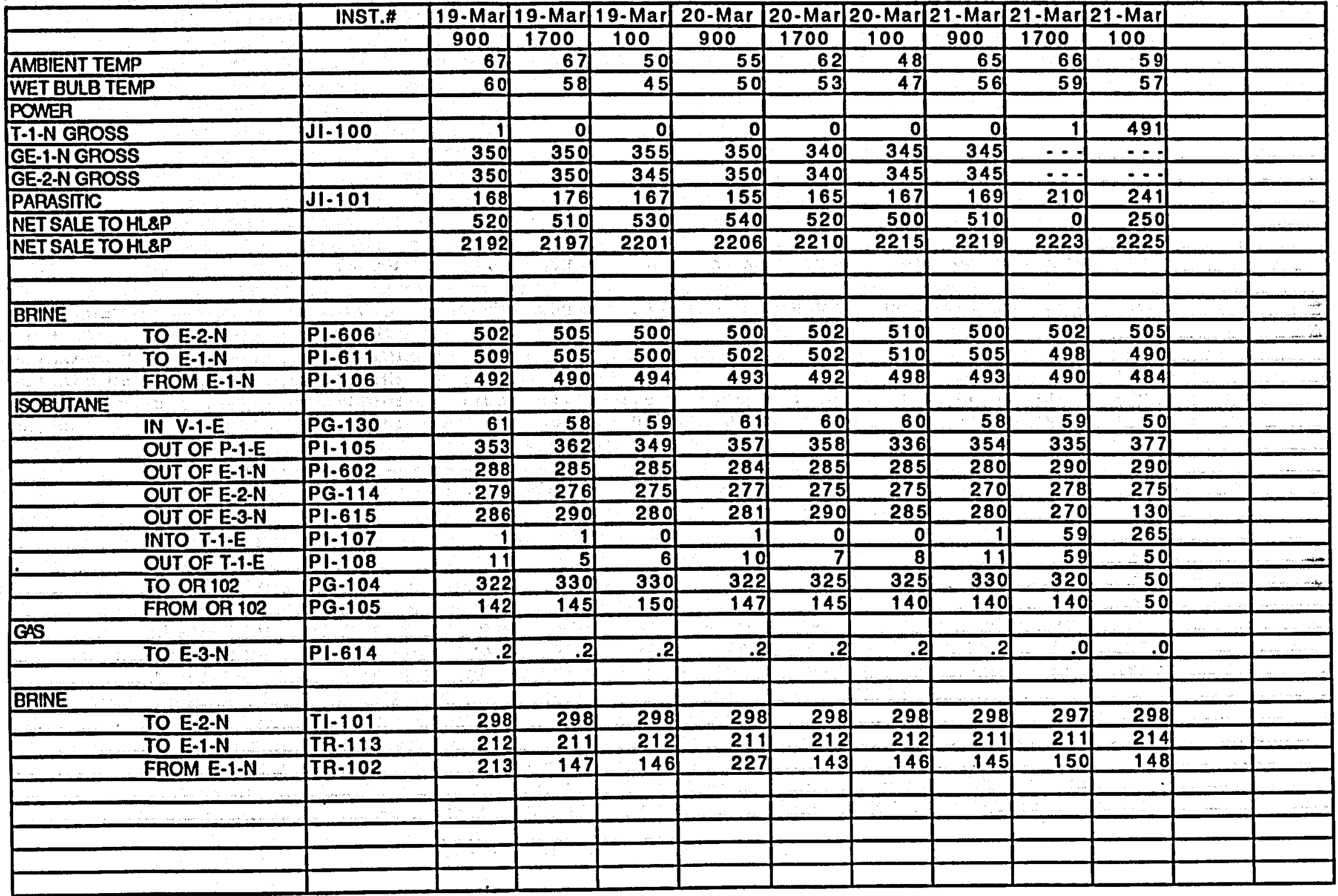


March 19-21, 1990

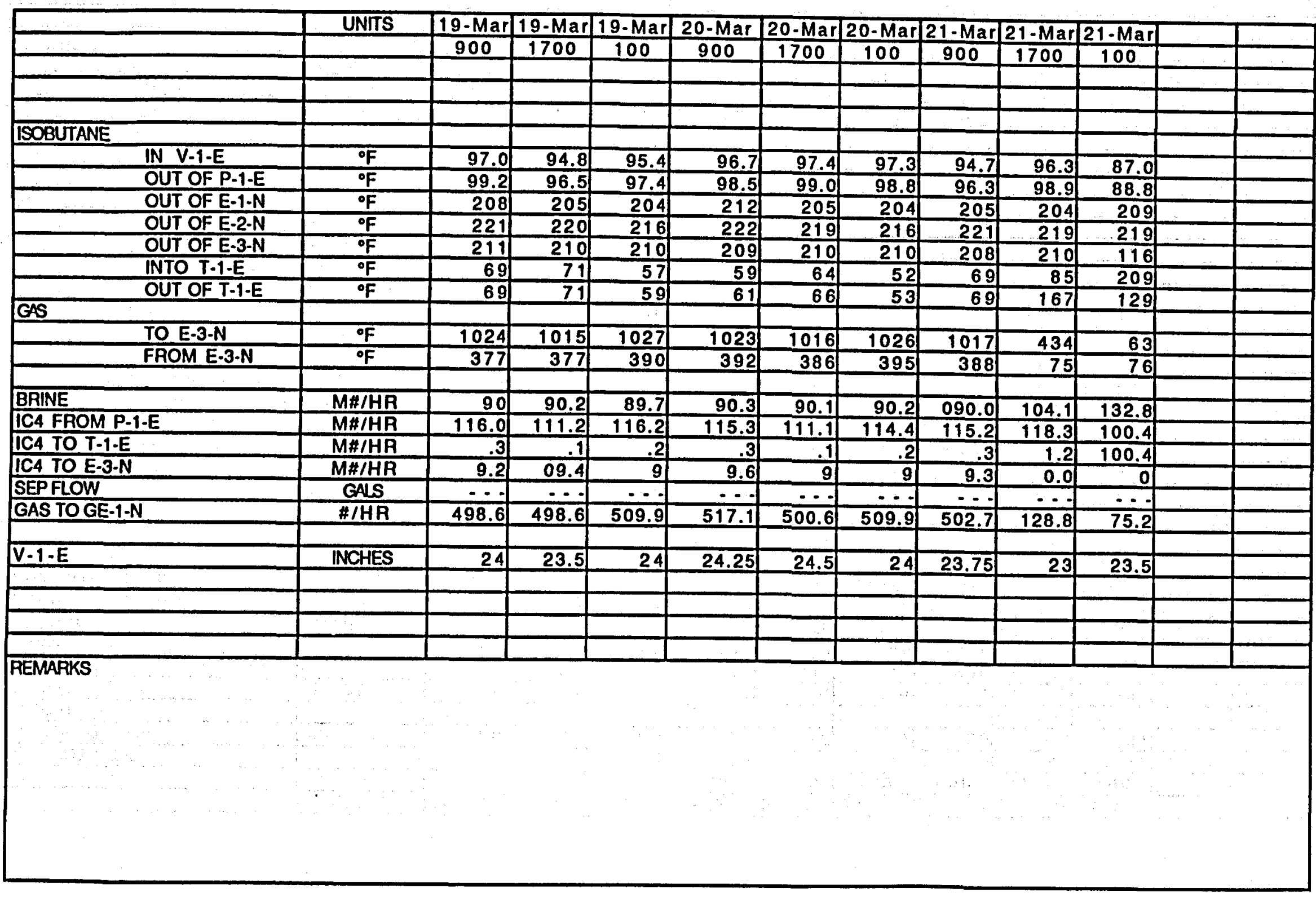


March 22-25, 1990

\begin{tabular}{|c|c|c|c|c|c|c|c|c|c|c|c|c|c|c|}
\hline & UNTS & INST.\# & 22-Mar & 22-Marl: & 22-Mar & 23-Mar & 23-Mar & 23-Marl: & 24-Mart & 24-Mar & 24-Mar & 25-Mar & 25-Marl & 25-Mar \\
\hline & & & 900 & 1700 & 100 & \begin{tabular}{|l|}
900 \\
\end{tabular} & 1700 & 100 & 900 & 1700 & 100 & 900 & 1700 & 100 \\
\hline AMBIENT TEMP & of & & 70 & 74 & 63 & 75 & 73 & 65 & 77 & 73 & 62 & 65 & 69 & 62 \\
\hline WET BULB TEMP & of & & 63 & 67 & 61 & 69 & 67 & 64 & 72 & 68 & 57 & 59 & 63 & 58 \\
\hline \multicolumn{15}{|l|}{$\begin{array}{l}\text { WEI BULB IEMP } \\
\text { POWER }\end{array}$} \\
\hline T-1-N GROSS & $\mathrm{KW}$ & $\pi 1.100$ & 484 & 522 & 530 & 515 & 512 & 0 & 0 & of & of & 0 & 의 & 의 \\
\hline GE-1-N GROSS & $\mathrm{KW}$ & & 0 & 345 & 345 & 345 & 345 & 345 & 345 & 340 & 340 & 340 & 335 & 340 \\
\hline GE-2-N GROSS & $\overline{K W}$ & & 0 & 345 & 345 & 345 & 345 & 345 & 345 & 345 & 340 & 345 & 345 & 340 \\
\hline PARASITIC & KW & ग1-101 & 250 & 250 & 251 & 249 & 252 & 182 & 191 & 192 & 192 & 179 & 177 & 180 \\
\hline NET SALE TOHL\&P & $\overline{K W}$ & & 260 & 910 & 930 & 910 & 920 & 500 & 500 & 500 & 500 & 500 & 500 & 500 \\
\hline \multirow{3}{*}{ NET SALE TO HL\&P } & FDG & & 2227 & 2230 & 2240 & 2248 & 2256 & 2262 & 2267 & 227.1 & 2275 & 2280 & 2284 & 2280 \\
\hline & $\mathrm{KW} \cdot \mathrm{HR}$ & & & & & & & & & & & & & \\
\hline & & & & & & & & & & & & & & \\
\hline BRINE & & & & & & & & & & & & & & \\
\hline TO E-2-N & PSIG & $P \mid-606$ & 500 & 500 & 502 & 500 & 502 & 504 & 500 & 502 & 505 & 505 & 505 & 500 \\
\hline TOE-1.N & PSIG & PI-611 & 495 & 486 & 490 & 490 & 489 & 508 & 505 & 502 & 510 & 505 & 505 & 505 \\
\hline FROM E-1-N & PSIG & $\mathrm{PI}-106$ & 487 & 474 & 478 & 477 & 478 & 491 & 492 & 493 & 495 & 494 & 495 & 494 \\
\hline \multicolumn{15}{|l|}{$\begin{array}{l}\text { FROM E-1.N } \\
\text { ISOBUTANE }\end{array}$} \\
\hline IN V.1.E & PSIG & PG-130 & 50 & 58 & 57 & 59 & 59 & 60 & 60 & 60 & 55 & 58 & 63 & 58 \\
\hline OUT OF P-1-E & PSIG & PI.105 & 375 & 363 & 337 & 349 & 356 & 343 & 337 & 358 & 340 & 348 & 359 & 358 \\
\hline OUT OF E-1-N & PSIG & $\mathrm{P} 1.602$ & 295 & 300 & 300 & 300 & 297 & 269 & 280 & 278 & 280 & 275 & 278 & 275 \\
\hline OUT OF E-2-N & PSIG & PG-114 & 275 & 280 & 280 & 280 & 298 & 260 & 270 & 269 & 270 & 268 & 270 & 270 \\
\hline OUT OF E-3-N & PSIG & $P 1.615$ & 260 & 289 & 300 & 285 & 285 & 268 & 275 & 274 & 280 & 275 & 275 & 280 \\
\hline INTO T-1.E & PSIG & $\mathrm{PI}-107$ & 264 & 265 & 267 & 266 & 265 & 37 & $\cdots$ & $\cdots$ & $\cdots$ & $\cdots$ & $\cdots$ & $\cdots$ \\
\hline OUT OF T-1-E & PSIG & $P \mid-108$ & 51 & 60 & 59 & 62 & 60 & 52 & $\ldots$ & $\ldots$ & & $\cdots$ & $\cdots$ & $\cdots$ \\
\hline TO OA 102 & PSIG & PG-104 & 50 & 58 & 55 & 55 & 58 & 147 & 330 & 330 & 325 & 140 & 145 & 195 \\
\hline FROM OA 102 & PSIG & PG.105 & 50 & 58 & 55 & 55 & 58 & 340 & 145 & 142 & 140 & 330 & 340 & 330 \\
\hline \multicolumn{15}{|l|}{$\begin{array}{l}\text { FROM OA } 102 \\
\text { GAS }\end{array}$} \\
\hline TO E-3-N & PSIG & PI.614 & .0 & .1 & .2 & .2 & .2 & .2 & .2 & .2 & .2 & .2 & .2 & \\
\hline & & & & & & & & & & & & & & \\
\hline \multicolumn{15}{|l|}{ TO E-3-N } \\
\hline TO E-2-N & ${ }^{\circ} \mathrm{F}$ & $T 1.101$ & 298 & 298 & 298 & 298 & 298 & 298 & 298 & 298 & 298 & 298 & 298 & 298 \\
\hline TO E-1.N & of & TR.113 & 213 & 215 & 214 & 215 & 214 & 209 & 210 & 209 & 209 & 209 & 210 & 210 \\
\hline FROM E-1.N & of & TR-102 & 152 & 148 & 148 & 174 & 148 & 147 & 148 & 153 & 146 & 145 & 149 & 146 \\
\hline \multirow{2}{*}{\multicolumn{15}{|c|}{ 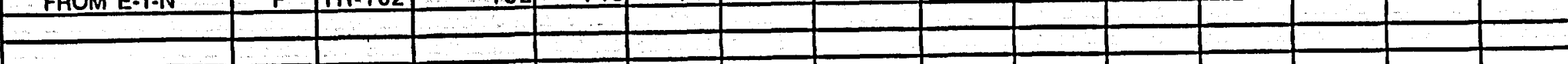 }} \\
\hline & & & & & & & & & & & & & & \\
\hline & & & & & & & & & & & & & & \\
\hline & & & & & & & & & & & & & & \\
\hline & & & & & & & & & & & & & & \\
\hline
\end{tabular}




\section{GEOPRESSURED HYBRID POWER SYSTEM - MANAGEMENT REPORT DATA}

March 22-25, 1990

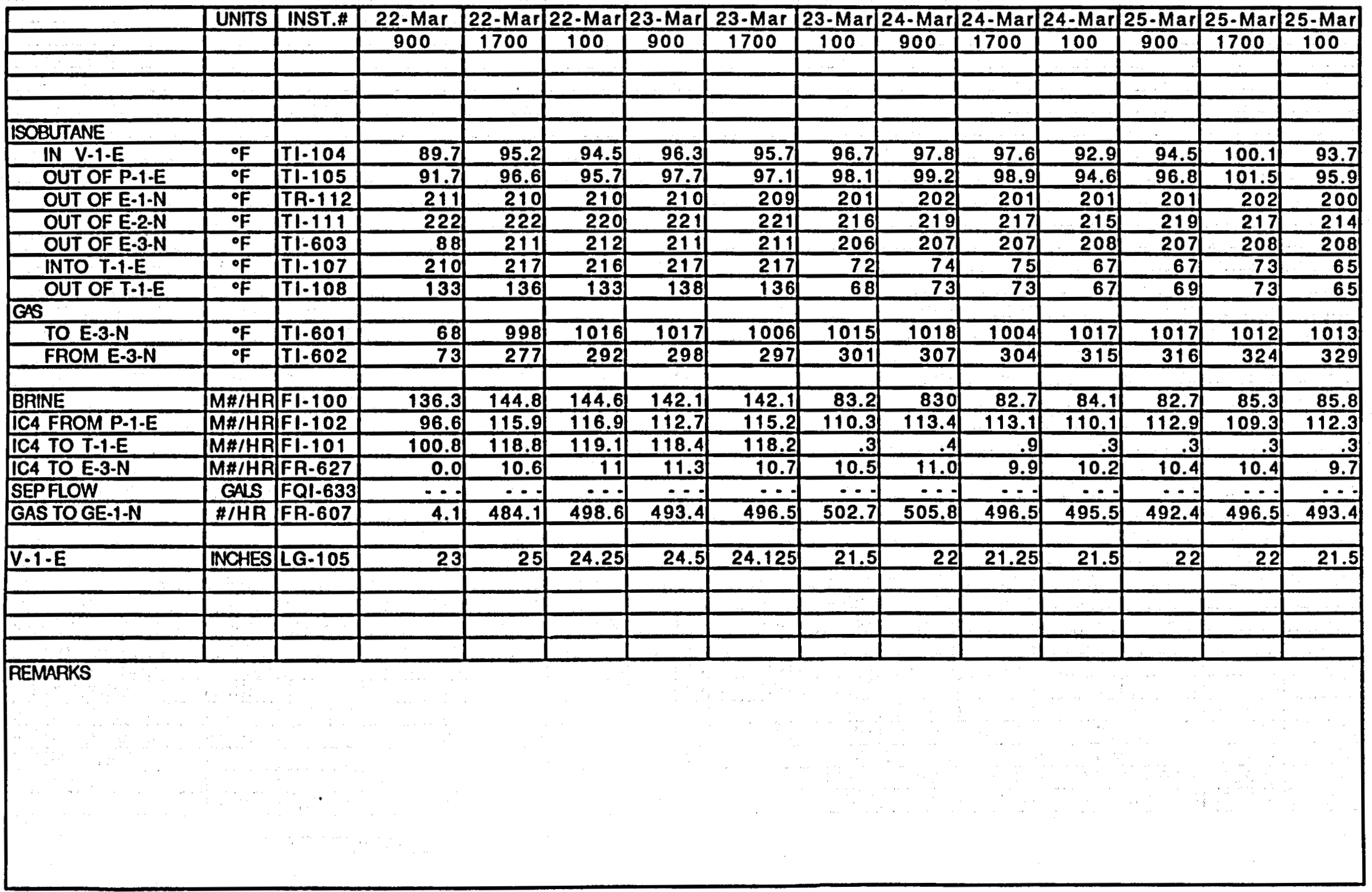


March 26-28, 1990

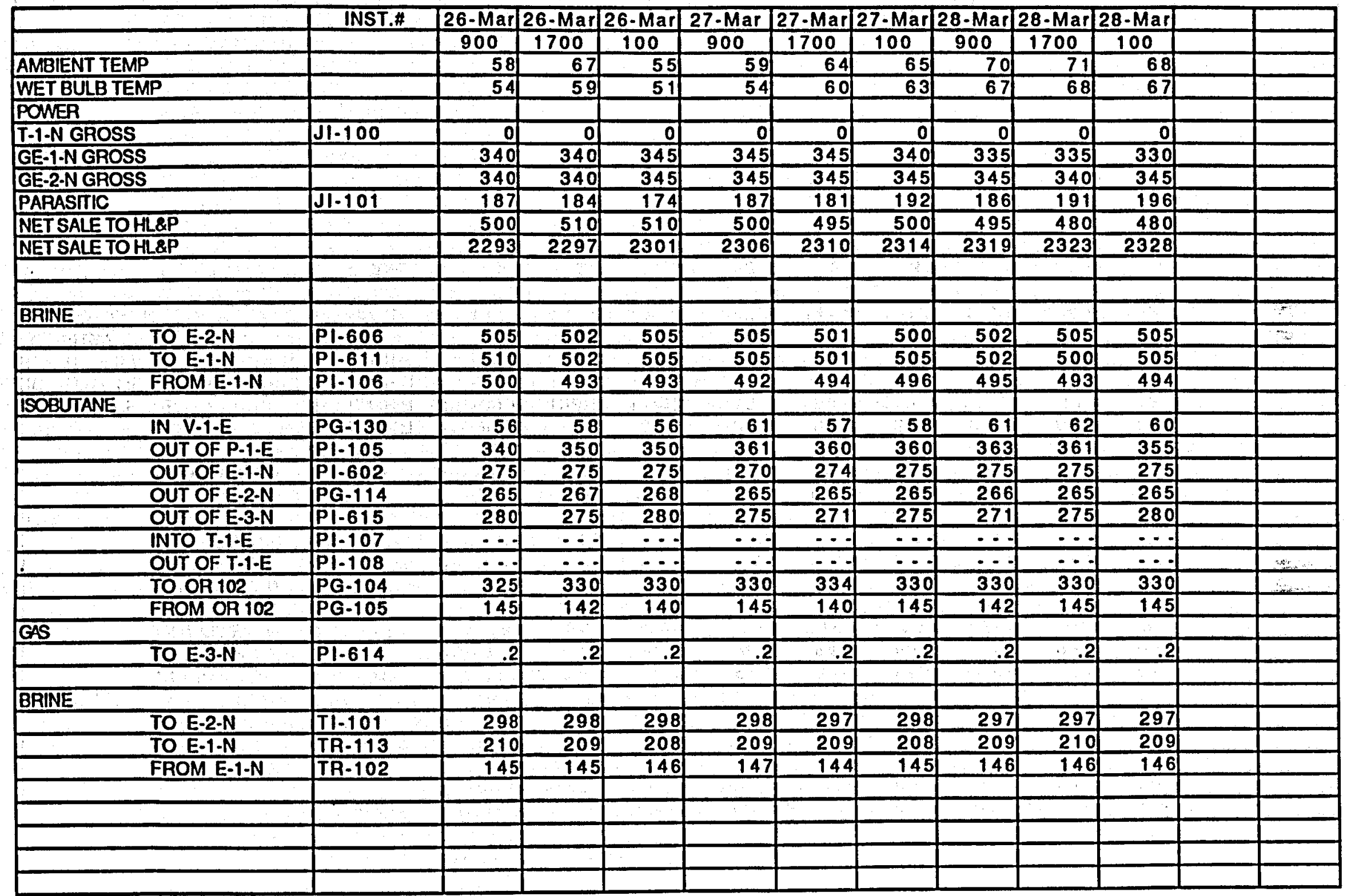


March 26-28, 1990

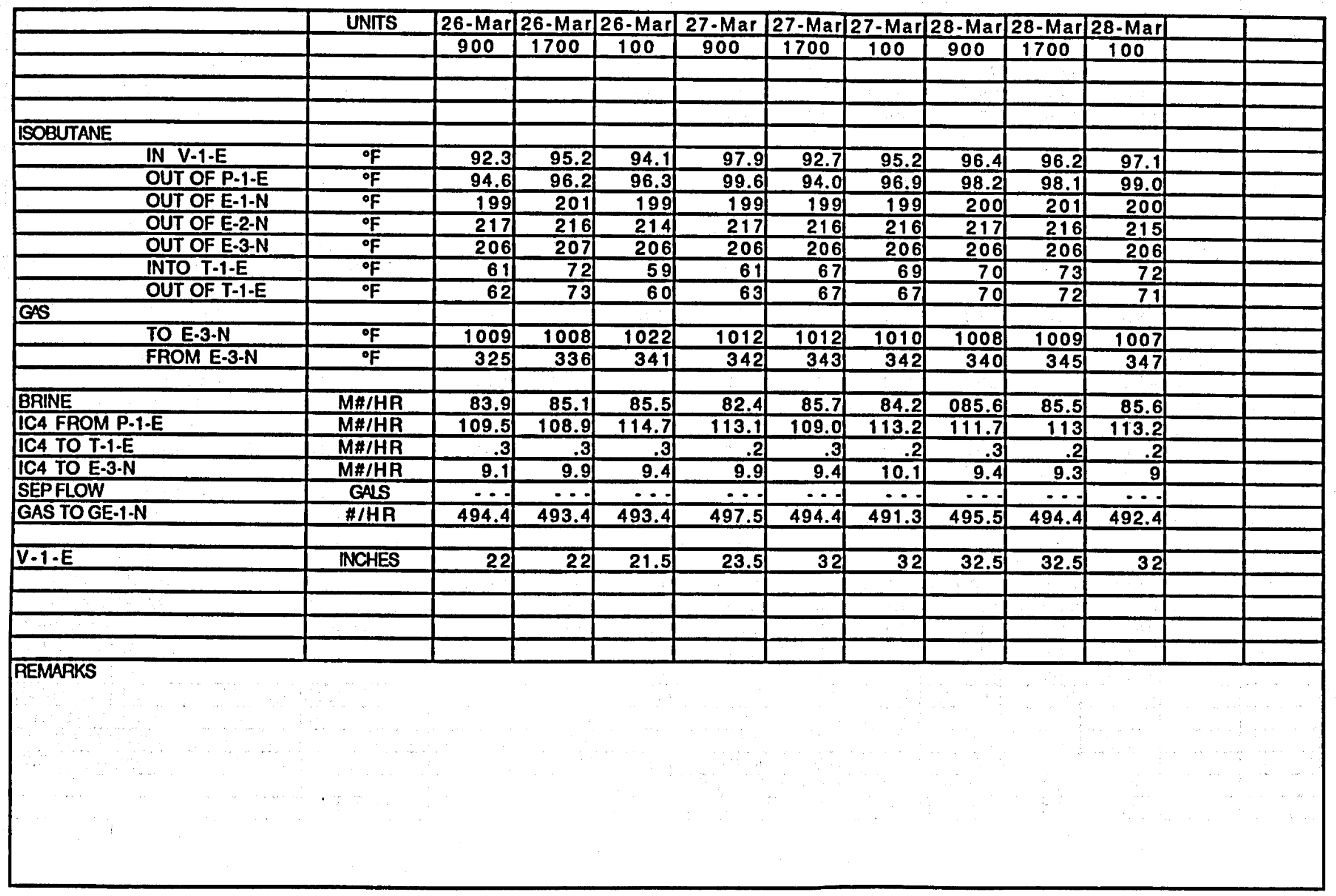


March 29-April 1, 1990

\begin{tabular}{|c|c|c|c|c|c|c|c|c|c|c|c|c|c|c|}
\hline & UNITS & INST.\# & 29-Mar & 29-Mar & 29-Mar & 30-Mar & 30-Mar & 30-Mar & 31-Mar & 31-Mar & 31-Mar & 1-Apr & 1-Apr & 1-Apr \\
\hline & & & 900 & 1700 & 100 & 900 & 1700 & 100 & 900 & 1700 & 100 & 900 & 1700 & 100 \\
\hline AMBIENT TEMP & of & & 70 & 60 & 63 & 65 & 73 & 64 & 65 & 74 & 67 & 69 & 68 & 67 \\
\hline WET BULB TEMP & ${ }^{\circ} \mathrm{F}$ & & 69 & 60 & 63 & 65 & 68 & 62 & 61 & 69 & 66 & 67 & 68 & 66 \\
\hline \multicolumn{15}{|l|}{ POWER } \\
\hline T-1-N GROSS & KW & $\mathrm{Jl} .100$ & 1 & 449 & 515 & 508 & 0 & 518 & 514 & 504 & 516 & 508 & 495 & 512 \\
\hline GE-1-N GROSS & KW & & 335 & 325 & 325 & 335 & 320 & 340 & 335 & 335 & 335 & 325 & 320 & 320 \\
\hline GE-2-N GROSS & KW & & 345 & 335 & 340 & 345 & 340 & 340 & 345 & 345 & 340 & 345 & 340 & 340 \\
\hline PARASITIC & KW & J1.101 & 203 & 239 & 240 & 249 & 130 & 252 & 251 & 249 & 251 & 240 & 239 & 252 \\
\hline NET SALE TO HL\&P & $\overline{K W}$ & & 490 & 830 & 880 & 895 & 500 & 900 & 900 & 885 & 902 & 885 & 880 & 875 \\
\hline \multirow[t]{3}{*}{ NET SALETO HL $8 P$} & PDG & & 2332 & 2337 & 2344 & 2352 & 2356 & 2362 & 2370 & 2378 & 2386 & 2392 & 2400 & 2408 \\
\hline & $\overline{K W-H A}$ & & & & $\ldots$ & & 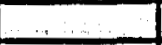 & & 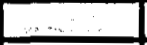 & $\ldots$ & $\ldots$ & $\pi$ & $\cdots$ & $\ldots$ \\
\hline & & & & & 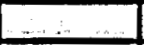 & & & & & & & & $\ldots$ & \\
\hline BRINE & & & & & & & & & & & & & & 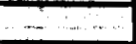 \\
\hline TO E-2.N & PSIG & PI.606 & 503 & 505 & 490 & 501 & 530 & 500 & 500 & 500 & 500 & 501 & 500 & 500 \\
\hline TO E-1.N & PSIG & $\mathrm{PI.611}$ & 505 & 500 & 490 & 491 & 530 & 490 & 489 & 484 & 485 & 490 & 490 & 490 \\
\hline FROM E-1-N & PSIG & $\mathrm{PI}-106$ & 494 & 500 & 475 & 477 & 529 & 480 & 479 & 475 & 478 & 477 & 475 & 475 \\
\hline \multicolumn{15}{|l|}{ ISOBUTANE } \\
\hline IN V-1.E & PSIG & PG-130 & 60 & 52 & 57 & 59 & 36 & 60 & 59 & 63 & 60 & 62 & 62 & 61 \\
\hline OUT OF P-1-E & PSIG & P1. 105 & 363 & 308 & 352 & 352 & 39 & 345 & 341 & 358 & 353 & 352 & 360 & 359 \\
\hline OUT OFE-1-N & PSIG & $\mathrm{P} 1.602$ & 275 & 285 & 297 & 297 & 110 & 300 & 296 & 300 & 300 & 299 & 300 & 300 \\
\hline OUT OF E-2.N & PSIG & PG.114 & 264 & 270 & 276 & 277 & 35 & 280 & 278 & 281 & 280 & 280 & 280 & 280 \\
\hline OUT OF E-3-N & PSIG & $P \mid \cdot 615$ & 270 & 280 & 300 & 282 & 160 & 290 & 285 & 287 & 290 & 286 & 282 & 290 \\
\hline INTO T-1.E & PSIG & PI.107 & $\cdots$ & 268 & 264 & 264 & 33 & 265 & 263 & 267 & 268 & 267 & 265 & 267 \\
\hline OUT OF T-1-E & PSIG & PI.108 & $\ldots$ & 54 & 60 & 62 & 36 & 62 & 61 & 65 & 64 & 65 & 66 & 64 \\
\hline TO OR 102 & PSIG & PG-104 & 328 & 290 & 55 & 59 & 35 & 60 & 59 & 62 & 60 & 61 & 60 & 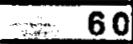 \\
\hline FROM OA 102 & PSIG & PG-105 & 142 & 130 & 55 & 59 & 35 & 60 & 59 & 61 & 60 & 61 & 60 & $-\infty$ \\
\hline \multicolumn{15}{|l|}{ GAS } \\
\hline TO E-3-N & PSIG & PI.614 & & .2 & .2 & .2 & .0 & .2 & .2 & 2 & .2 & .2 & .2 & 2 \\
\hline &. & & & & & & $\cdots$ & & & & & & & \\
\hline \multicolumn{15}{|l|}{ BRINE } \\
\hline TO E-2-N & ${ }^{\circ} \mathrm{F}$ & $T \mid-101$ & 297 & 297 & 297 & 298 & 291 & 298 & 298 & 297 & 298 & 298 & 298 & 298 \\
\hline TO E-1-N & OF & TA-113 & 209 & 212 & 215 & 214 & 074 & 215 & 214 & 215 & 215 & 214 & 214 & 215 \\
\hline FAOM E-1-N & $\circ \mathrm{F}$ & TR-102 & 154 & 143 & 147 & 148 & 103 & 143 & 143 & 143 & 145 & 146 & 145 & 145 \\
\hline & & & & & & & & & & & & & & \\
\hline & & & $=$ & & & & & & & & & & & \\
\hline & & & & & & & & & & & & & & \\
\hline & & & & & & & & & & & & & & \\
\hline & & & & & & & & & & & & & & \\
\hline
\end{tabular}


GEOPRESSUAED HYBRID POWER SYSTEM - MANAGEMENT REPORT DATA

March 29-April 1, 1990

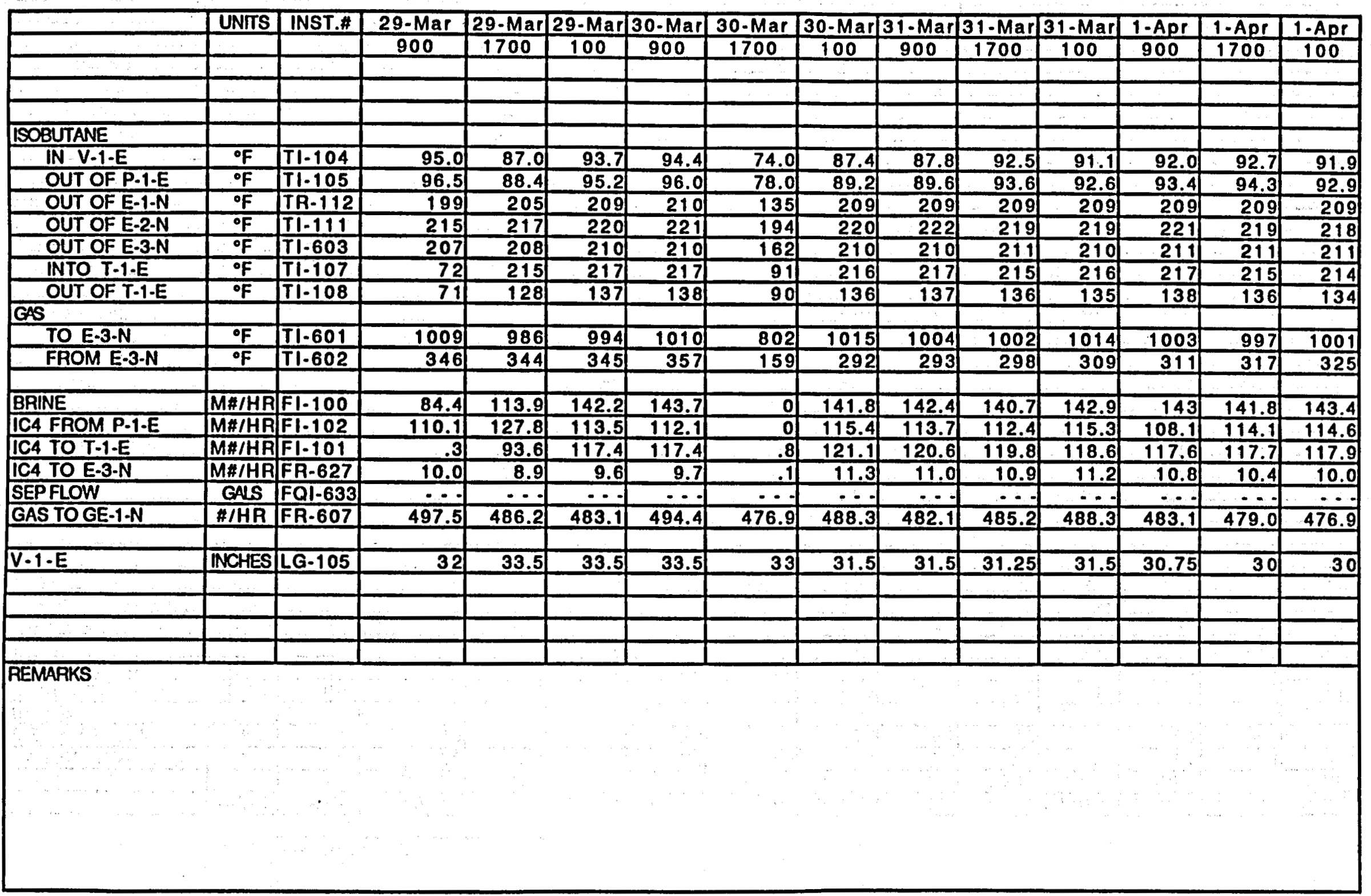


April 2-4, 1990

\begin{tabular}{|c|c|c|c|c|c|c|c|c|c|c|c|c|}
\hline & INST.\# & 2-Apr & 2-Apr & 2-Apr & 3-Apr & 3.Apr & 3.Apr & 4-Apr & 4-Apr & 4-Apr & & \\
\hline & & 900 & 1700 & 100 & 900 & 1700 & 100 & 900 & 1700 & 100 & & \\
\hline AMBIENT TEMP & & 70 & 76 & 64 & 60 & 73 & 57 & 64 & 76 & 60 & & \\
\hline WET BULB TEMP & & 69 & 69 & 60 & 53 & 62 & 55 & 60 & 66 & 59 & & \\
\hline POWER & & & & & & & & & & & & \\
\hline T-1-N GROSS & $J 1.100$ & 511 & 498 & 524 & 544 & 535 & 544 & 0 & 509 & 525 & & \\
\hline GE-1-N GROSS & & 325 & 120 & 330 & 330 & 335 & 330 & 335 & 330 & 330 & & \\
\hline GE-2-N GROSS & & 345 & 333 & 340 & 345 & 340 & 340 & 340 & 345 & 340 & & \\
\hline PARASTIC & J1.101 & 250 & 250 & 242 & 253 & 240 & 254 & 238 & 239 & 243 & & \\
\hline NET SALE TO HL\&P & & 895 & 650 & 900 & 922 & 920 & 900 & 450 & 900 & 900 & & \\
\hline NET SALE TO HL \&P & & 2415 & 2423 & 2428 & 2436 & 2444 & 2452 & 2456 & 2462 & 2470 & & \\
\hline & & & & & & & & & & & & \\
\hline BRINE & & & & & & & & & & & & \\
\hline TO E-2-N & PI.606 & 500 & 504 & 500 & 501 & 500 & 500 & 505 & 510 & 500 & & \\
\hline TO E-1-N & $\mathrm{P} \mid-611$ & 490 & 492 & 490 & 491 & 490 & 490 & 509 & 495 & 490 & & \\
\hline FROM E-1-N & PI.106 & 475 & 478 & 477 & 479 & 405 & 483 & 493 & 478 & 483 & & \\
\hline ISOBUTANE & & & & & & & & & & & & \\
\hline IN $V-1-E$ & PG.130 & 63 & 64 & 59 & 55 & 57 & 58 & 48 & 58 & 56 & & \\
\hline OUT OF P-1-E & $P 1-105$ & 351 & 372 & 349 & 365 & 363 & 343 & 348 & 375 & 334 & & \\
\hline OUT OF E-1.N & $P \mid-602$ & 304 & 300 & 300 & 299 & 300 & 300 & 278 & 295 & 300 & & \\
\hline OUT OFE-2.N & $P G-114$ & 284 & 280 & 280 & 281 & 280 & 280 & 270 & 275 & 280 & & \\
\hline OUT OFE-3-N & PI-615 & 289 & 290 & 290 & 284 & 290 & 290 & 278 & 285 & 290 & & \\
\hline INTO T-1-E & $\mathrm{P} \mid \cdot 107$ & 268 & 269 & 268 & 263 & 266 & 267 & 49 & 260 & 264 & & \\
\hline OUT OF T-1-E & $P \mid-108$ & 65 & 65 & 60 & 55 & 58 & 56 & 51 & 60 & 59 & & \\
\hline TO OR 102 & PG-104 & 61 & 61 & 60 & 61 & 55 & 55 & 325 & 60 & 55 & & \\
\hline FROM OA 102 & PG.105 & 61 & 60 & 60 & 61 & 55 & 55 & 135 & 60 & 55 & & \\
\hline GAS & & & & & & & & & & & & \\
\hline TO E-3-N & PI-614 & .2 & .2 & .2 & .2 & .2 & .2 & .2 & .1 & .2 & & \\
\hline & & & & & & & & & & & & \\
\hline BAINE & & & & & & & & & & & & \\
\hline TOE-2-N & $T 1.101$ & 298 & 298 & 298 & 298 & 298 & 298 & 298 & 298 & 298 & & \\
\hline TO E-1.N & TR.113 & 215 & 215 & 215 & 214 & 215 & 215 & 210 & 214 & 214 & & \\
\hline FROM E-1-N & TR.102 & 145 & 146 & 145 & 144 & 144 & 144 & 138 & 143 & 159 & & \\
\hline & & & & & & & & & & & & \\
\hline & & & & & & & & & & & & \\
\hline & & & & & & & & & & & & \\
\hline & & & & & & & & & & & & \\
\hline & & & & & & & & & & & & \\
\hline
\end{tabular}


April 2.4, 1990

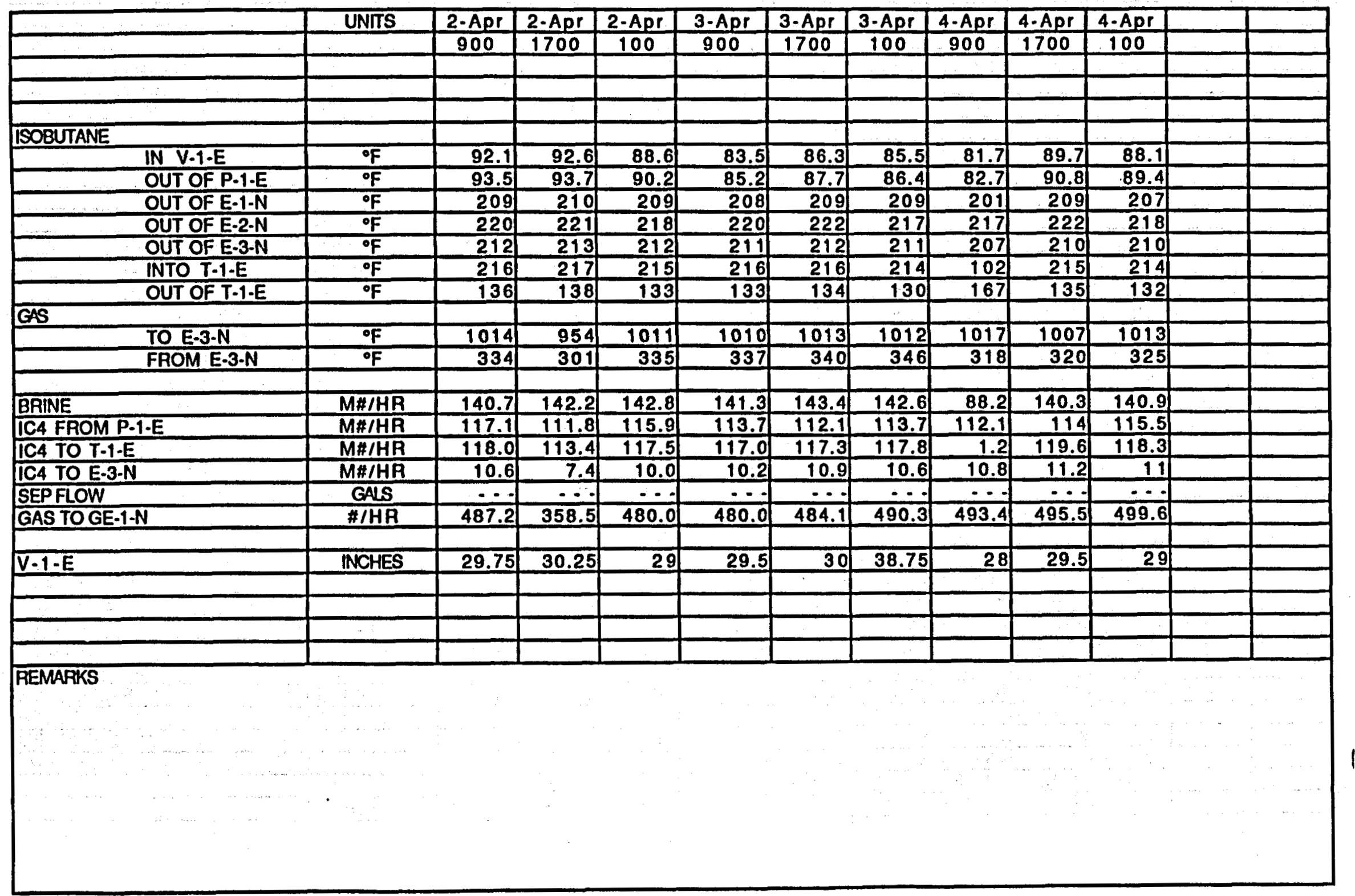


April 5-8, 1990

\begin{tabular}{|c|c|c|c|c|c|c|c|c|c|c|c|c|c|c|}
\hline & UNTS & INST.\# & 5-ApP & 5-Apr & 5-Apr & 6-Apr & 6-Apr & 6-Apr & 7-Aprl & 7.Aprl & 7-Aprl & 8-Apr & B.Apr & 8-Apr \\
\hline & & & 900 & 1700 & 100 & 900 & 1700 & 100 & 900 & 1700 & 100 & 900 & 1700 & 100 \\
\hline AMBIENT TEMP & ${ }^{\circ} \mathrm{F}$ & & 72 & 77 & 68 & 65 & 61 & 50 & 53 & 62 & 54 & 60 & 67 & 65 \\
\hline WET BULB B TEMP & of & & 68 & 71 & 67 & 64 & 56 & 49 & 53 & 51 & 41 & 56 & 62 & 62 \\
\hline POWER & & & & & & & & & & & & & & \\
\hline T-1-N GROSS & $\mathrm{KW}$ & $\$ 1-100$ & 482 & 507 & 513 & 517 & 0 & 550 & 555 & 540 & 553 & 536 & 523 & 521 \\
\hline GE-1.N GROSS & $\mathrm{KW}$ & & $\cdots$ & $\cdots$ & $\ldots$ & 0 & 340 & 340 & 340 & 340 & 340 & 340 & 340 & 340 \\
\hline GE-2-NGROSS & $\mathrm{KW}$ & & 340 & 340 & 340 & 340 & 340 & 340 & 340 & 340 & 340 & 340 & 340 & 340 \\
\hline PARASTIC & $\mathrm{KW}$ & $J 1.101$ & 244 & 250 & 250 & 252 & 248 & 255 & 243 & 252 & 261 & 252 & 251 & 251 \\
\hline NET SALE TO HL\&P & $\mathrm{KW}$ & & 570 & 580 & 580 & 590 & 450 & 920 & 950 & 940 & 940 & $\because 930$ & 915 & 920 \\
\hline NET SALE TO HL\&P & FDG & & 2477 & 2482 & 2486 & 2492 & 2495 & 2503 & 2512 & 2520 & 2528 & 2537 & 2542 & 2550 \\
\hline & KW-HA & & & & & & & & & & & & & \\
\hline & & & & & & & & & & & & & & \\
\hline BRINE & & & & & & & & & & & & & & \\
\hline TO E-2-N & PSIG & P1.606 & 505 & 505 & 505 & 500 & 505 & 500 & 500 & 505 & 500 & 500 & 505 & 500 \\
\hline TO E-1-N & PSIG & PI.611 & 495 & 490 & 490 & 490 & 512 & 490 & 495 & 490 & 490 & 490 & 490 & 490 \\
\hline FROM E.1-N & PSIG & PI.106 & 476 & 475 & 479 & 475 & 503 & 484 & 482 & 481 & 479 & 480 & 480 & 480 \\
\hline ISOBUTANE & & & & & & & & & & & & & & \\
\hline IN $V-1-E$ & PSIG & PG.130 & 61 & 63 & 60 & 60 & 52 & 52 & 48 & 53 & 52 & 55 & 58 & 60 \\
\hline OUT OF P-1-E & PSIG & $\mathrm{Pl}-105$ & 366 & 363 & 350 & 349 & 385 & 344 & 360 & 368 & 331 & 325 & 357 & 355 \\
\hline OUT OF E-1-N & PSIG & PI.602 & 295 & 300 & 300 & 305 & 185 & 300 & 295 & 295 & 295 & 298 & 295 & 300 \\
\hline OUT OF E-2-N & PSIG & $\mathrm{PG}-114$ & 275 & 280 & 280 & 285 & 180 & 275 & 280 & 280 & 280 & 280 & 280 & 280 \\
\hline OUT OF E-3-N & PSIG & $\mathrm{PI.615}$ & 285 & 290 & 290 & 290 & 170 & 290 & 285 & 285 & 290 & 295 & 290 & 290 \\
\hline INTO T-1-E & PSIG & $\mathrm{P} 1.107$ & 260 & 266 & 267 & 268 & 48 & 265 & 263 & 266 & 264 & 265 & 264 & 265 \\
\hline OUT OF T-1-E & PSIG & $\mathrm{PI.108}$ & 64 & 64 & 64 & 61 & 57 & 53 & 51 & 54 & 55 & 58 & 62 & 63 \\
\hline TO OR 102 & PSIG & PG.104 & 60 & 61 & 60 & 60 & 150 & 55 & 45 & 50 & 50 & 50 & 5.5 & \\
\hline FROM OA 102 & PSIG & PG-105 & 60 & 60 & 60 & 60 & 360 & 55 & 45 & 50 & 50 & 50 & 55 & 55 \\
\hline GAS & & & & & & & & & & & & & & \\
\hline TO E-3-N & PSIG & PI.614 & .2 & .2 & .2 & .2 & .1 & .2 & .2 & .2 & .2 & .2 & .2 & .2 \\
\hline & & & & & & & & & & & & & & \\
\hline BRINE & & & & & & & & & & & & & & \\
\hline TO E-2-N & ${ }^{\circ} \mathrm{F}$ & $T 1.101$ & 298 & 297 & 298 & 298 & 297 & 298 & 297 & 298 & 298 & 298 & 297 & 298 \\
\hline TOE-1-N & ${ }^{\circ} \mathrm{F}$ & TR.113 & 213 & 215 & 216 & 217 & 177 & 215 & 215 & 214 & 215 & 216 & 216 & 216 \\
\hline FROM E-1-N & ${ }^{\circ} \mathrm{F}$ & TR-102 & 149 & 148 & 190 & 149 & 114 & 142 & 142 & 143 & 143 & 147 & $\cdots$ & 14.7 \\
\hline & & & & & & & & & & & & & & \\
\hline & & & & & & & & & & & & & & \\
\hline & & & & & & & & & & & & & & \\
\hline & & & & & & & & & & & & & & \\
\hline & & & & & & & & & & & & & & \\
\hline
\end{tabular}


GEOPRESSURED HYBRID POWER SYSTEM - MANAGEMENT REPORT DATA

April $5-8,1990$

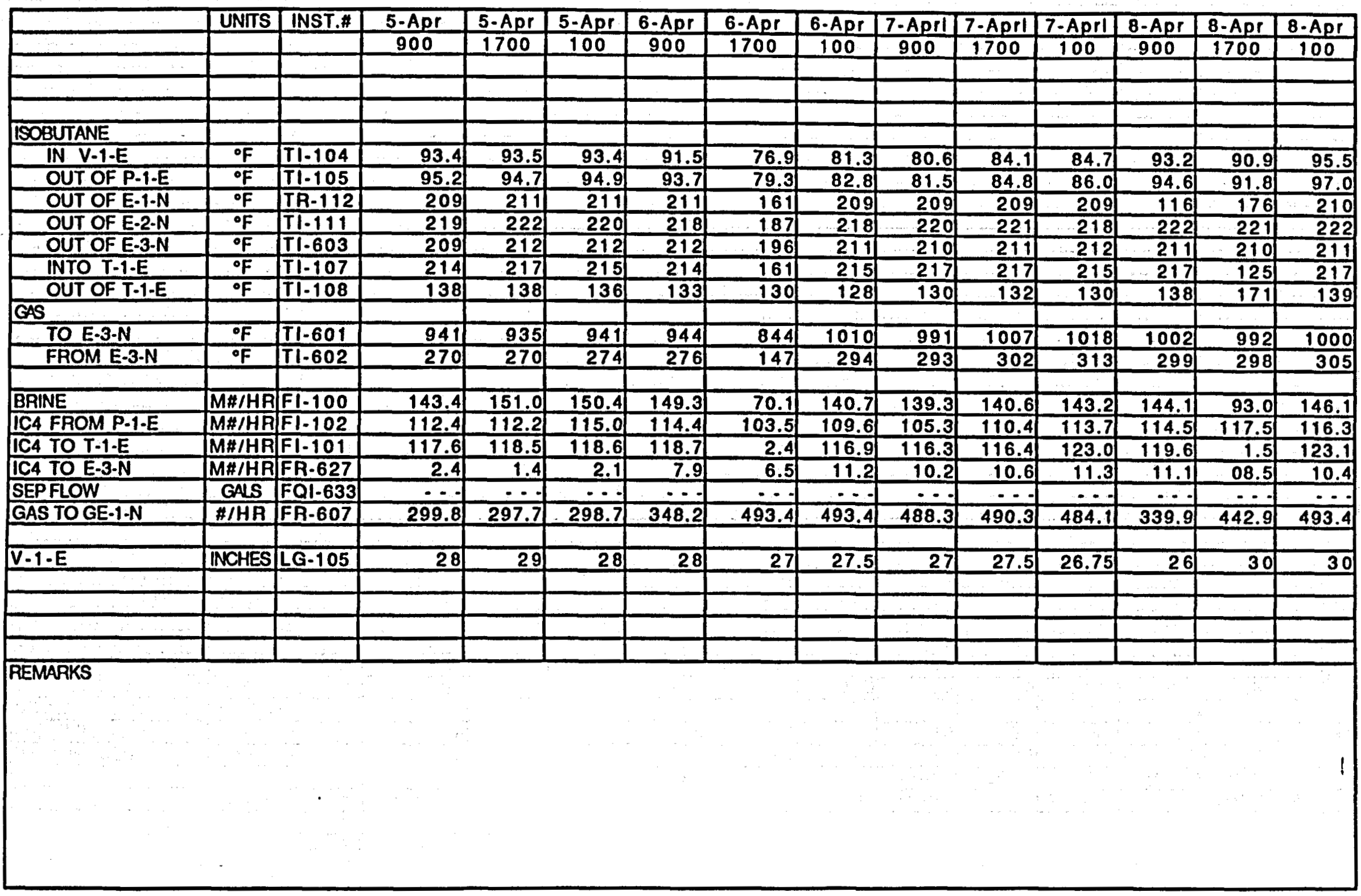


April 9-11, 1990

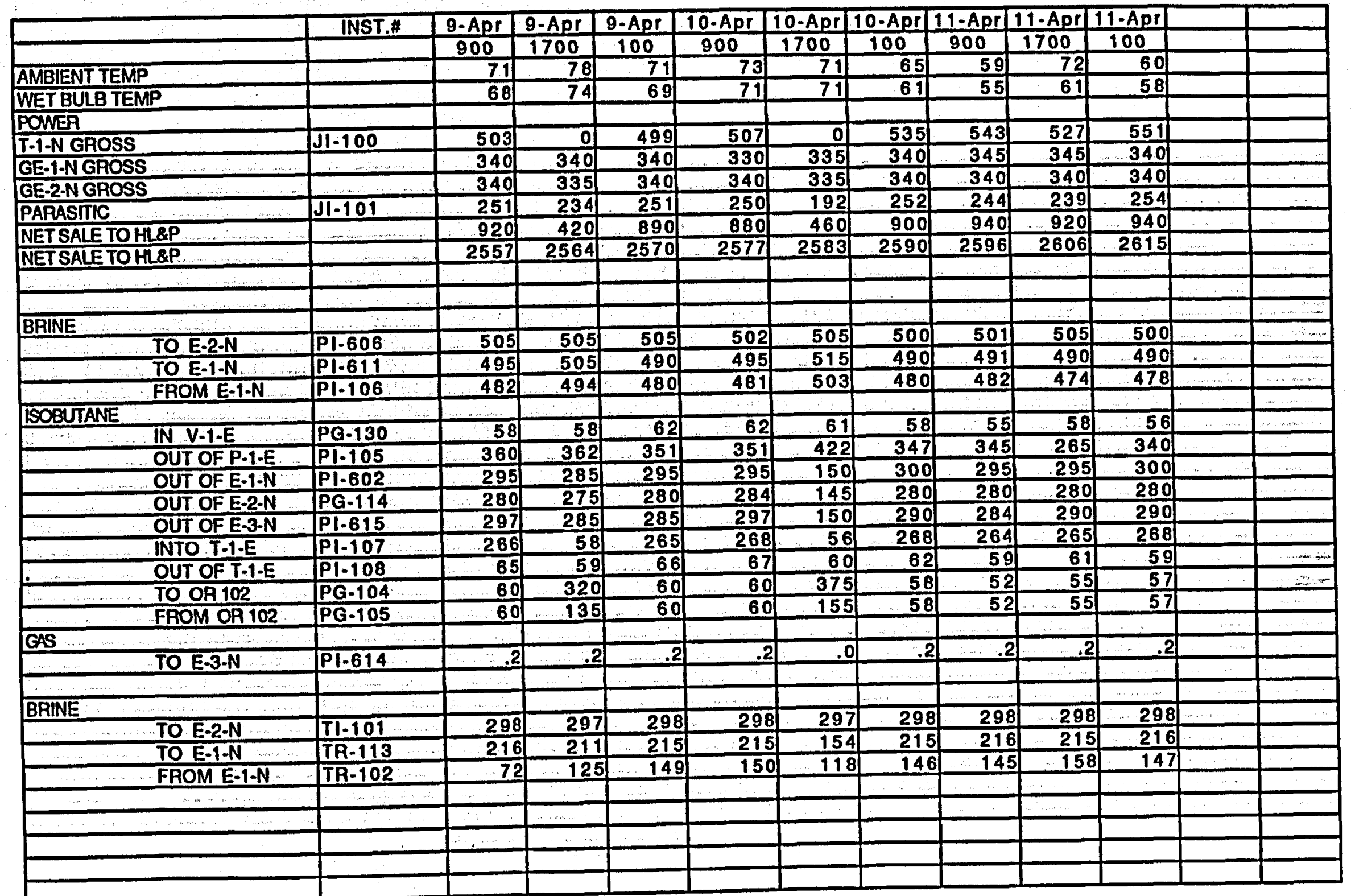


April 9-11, 1990

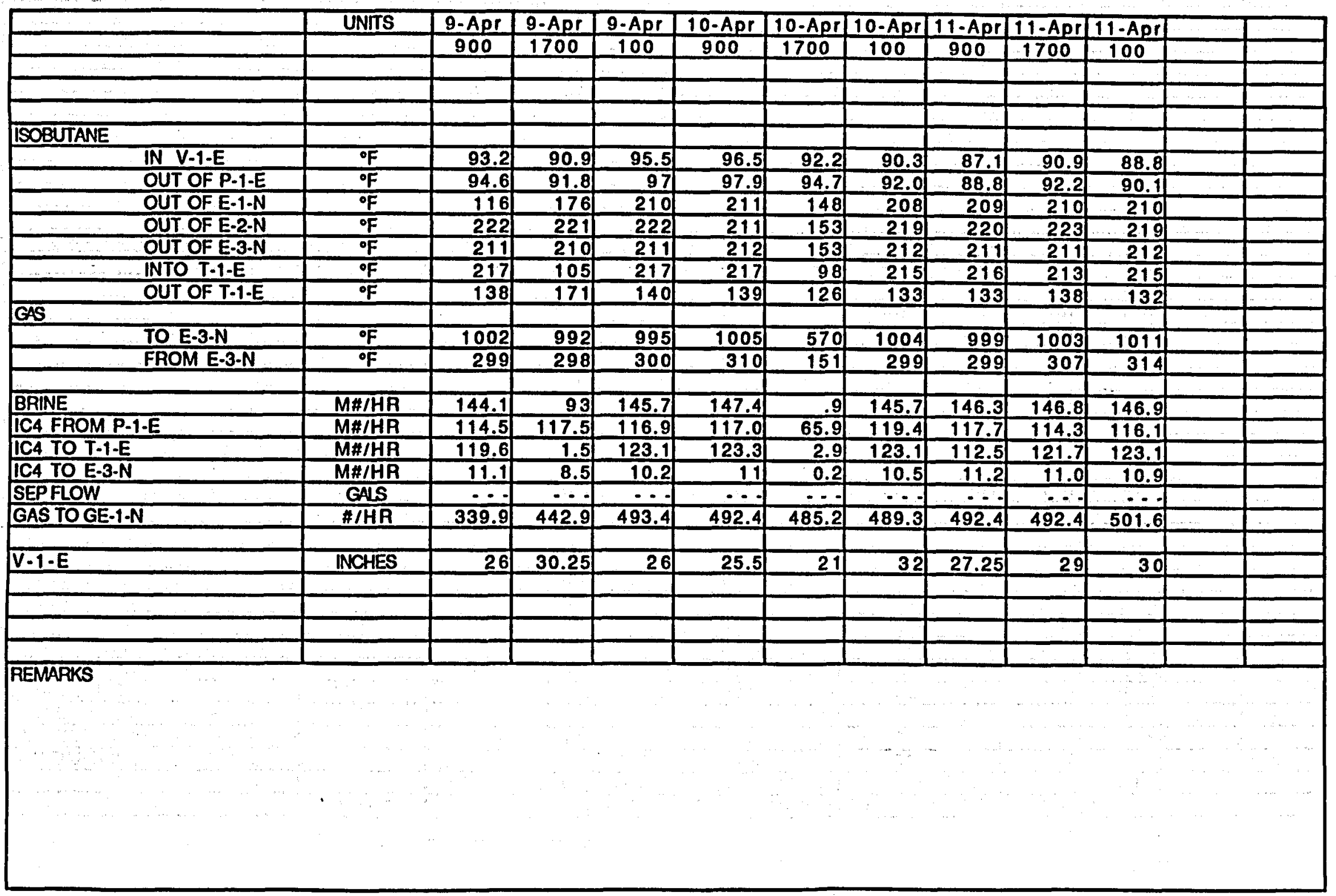


April 12-15, 1990

\begin{tabular}{|c|c|c|c|c|c|c|c|c|c|c|c|c|c|c|}
\hline & UNTSI & INST.\# & 12-Apr & 12-Apr & 12-Apr & 13-Apr & 13-Apr & 13-Apr & 14-Apr & 14-Apr & 14-Apr & 15-Apr & 15-Apr & 15-Apr \\
\hline & & & 900 & 1700 & 100 & 900 & 1700 & 100 & 900 & 1700 & 100 & 900 & 1700 & 100 \\
\hline AMBIENT TEMP & ${ }^{\circ} \mathrm{F}$ & & 62 & 70 & 62 & 72 & 70 & 66 & 74 & 74 & 61 & 77 & 79 & 64 \\
\hline WET BULB TEMP & $\circ^{\circ} \mathrm{F}$ & & 58 & 60 & 57 & 65 & 60 & 64 & 70 & 66 & 60 & 68 & 71 & 62 \\
\hline POWER & & & & & & & & & & & & & & \\
\hline$T-1-N$ GAOSS & $\overline{K W}$ & $J 1-100$ & 535 & 528 & 529 & 516 & 519 & 516 & 523 & 518 & 535 & 526 & 516 & 526 \\
\hline GE-1-N GROSS & $\overline{K W}$ & & 340 & 345 & 335 & 345 & 340 & 340 & 340 & 340 & 340 & 335 & 335 & 335 \\
\hline GE-2-NGROSS & $\overline{K W}$ & & 340 & 345 & 340 & 345 & 340 & 340 & 340 & 340 & 340 & 335 & 340 & 340 \\
\hline PARASITIC & $\mathrm{KW}$ & J1-101 & 253 & 252 & 252 & 240 & 251 & 251 & 241 & 249 & 242 & 251 & 250 & 253 \\
\hline NET SALE TO HL\&P & $\overline{K W}$ & & 940 & 920 & 920 & 910 & 910 & 900 & 905 & 900 & 920 & 900 & 900 & 900 \\
\hline NET SALE TO HL\&P & $\mathbf{F O G}$ & & 2623 & 2631 & 2639 & 2647 & 2655 & 2663 & 2671 & 2679 & 2687 & 2695 & 2703 & 2711 \\
\hline & $\mathrm{KW}-\mathrm{HA}$ & & & & & -1 & & & & & & & 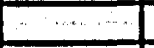 & \\
\hline & & & & & & & & & & & & & & \\
\hline BRINE & & & & & & & & & & & & & & \\
\hline TO E-2-N & PSIG & PI-606 & 500 & 505 & 500 & 505 & 505 & 505 & 503 & 509 & 510 & 503 & 510 & 505 \\
\hline TOE-1-N & PSIG & PI-611 & 490 & 490 & 490 & 491 & 490 & 490 & 492 & 490 & 500 & 494 & 495 & 495 \\
\hline FROM E-1-N & PSIG & PI.106 & 490 & 481 & 479 & 482 & 479 & 481 & 477 & 478 & 483 & 480 & 477 & 480 \\
\hline ISOBUTANE & & & & & & & & & & & & & & \\
\hline IN V-1-E & PSIG & PG-130 & 56 & 55 & 57 & 59 & 60 & 60 & 61 & 59 & 58 & 59 & 60 & 60 \\
\hline OUT OF P.1-E & PSIG & $P \mid-105$ & 313 & 350 & 353 & 354 & 350 & 358 & 357 & 330 & 340 & 353 & 360 & 331 \\
\hline OUT OF E-1-N & PSIG & $\mathrm{PI}-602$ & 295 & 295 & 295 & 294 & 298 & 300 & 299 & 301 & 300 & 289 & 300 & 300 \\
\hline OUT OF E-2-N & PSIG & $P G-114$ & 280 & 278 & 275 & 277 & 280 & 280 & 282 & 285 & 280 & 283 & 280 & 280 \\
\hline OUT OFE-3-N & PSIG & P1.615 & 290 & 285 & 285 & 280 & 290 & 290 & 289 & 290 & 290 & 289 & 290 & 290 \\
\hline INTO T-1-E & PSIG & $P \mid-107$ & 264 & 263 & 263 & 262 & 265 & 266 & 267 & 266 & 269 & 265 & 265 & 266 \\
\hline OUT OF T-1-E & PSIG & $\mathrm{PI}-108$ & 60 & 60 & 60 & 62 & 64 & 64 & 65 & 63 & 62 & 62 & 6.4 & 62 \\
\hline TO OA 102 & PSIG & PG.104 & 50 & 55 & 55 & 58 & 60 & 60 & 60 & 60 & 60 & 58 & 60 & 60 \\
\hline FROM OR 102 & PSIG & PG-105 & 50 & 55 & 55 & 57 & 60 & 60 & 60 & 60 & 60 & 58 & 60 & 60 \\
\hline GAS & & & & & & & & & & & & & & \\
\hline TO E.3.N & PSIG & $\overline{P I .614}$ & .2 & .2 & .2 & .2 & .2 & .2 & .2 & .2 & .2 & .2 & .2 & 2 \\
\hline & & & & 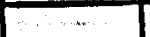 & & & & & & & & & & \\
\hline BRINE & & & & & & & & & & & & & & \\
\hline TO E-2-N & ${ }^{\circ} \mathrm{F}$ & $T 1-101$ & 298 & 297 & 298 & 298 & 298 & 298 & 298 & 298 & 298 & 298 & 298 & 298 \\
\hline TO E-1-N & $\circ \mathrm{F}$ & TR.113 & 215 & 215 & 215 & 215 & 216 & 216 & 216 & 216 & 216 & 216 & 216 & $\cdots$ \\
\hline FROM E-1-N & $\circ \mathrm{F}$ & TR.102 & 148 & 146 & 147 & 147 & 173 & 151 & 151 & 201 & 150 & 201 & 220 & $\ldots$ \\
\hline & & & & & & & & & & & & & & \\
\hline & & & & & & & & & & & & & & \\
\hline & & & & & & & & & & & & & & \\
\hline & & & & & & & & & & & & & & \\
\hline & & & & & & & & & & & & & & \\
\hline
\end{tabular}


GEOPRESSURED HYBRID POWER SYSTEM - MANAGEMENT REPORT DATA

April 12-15, 1990

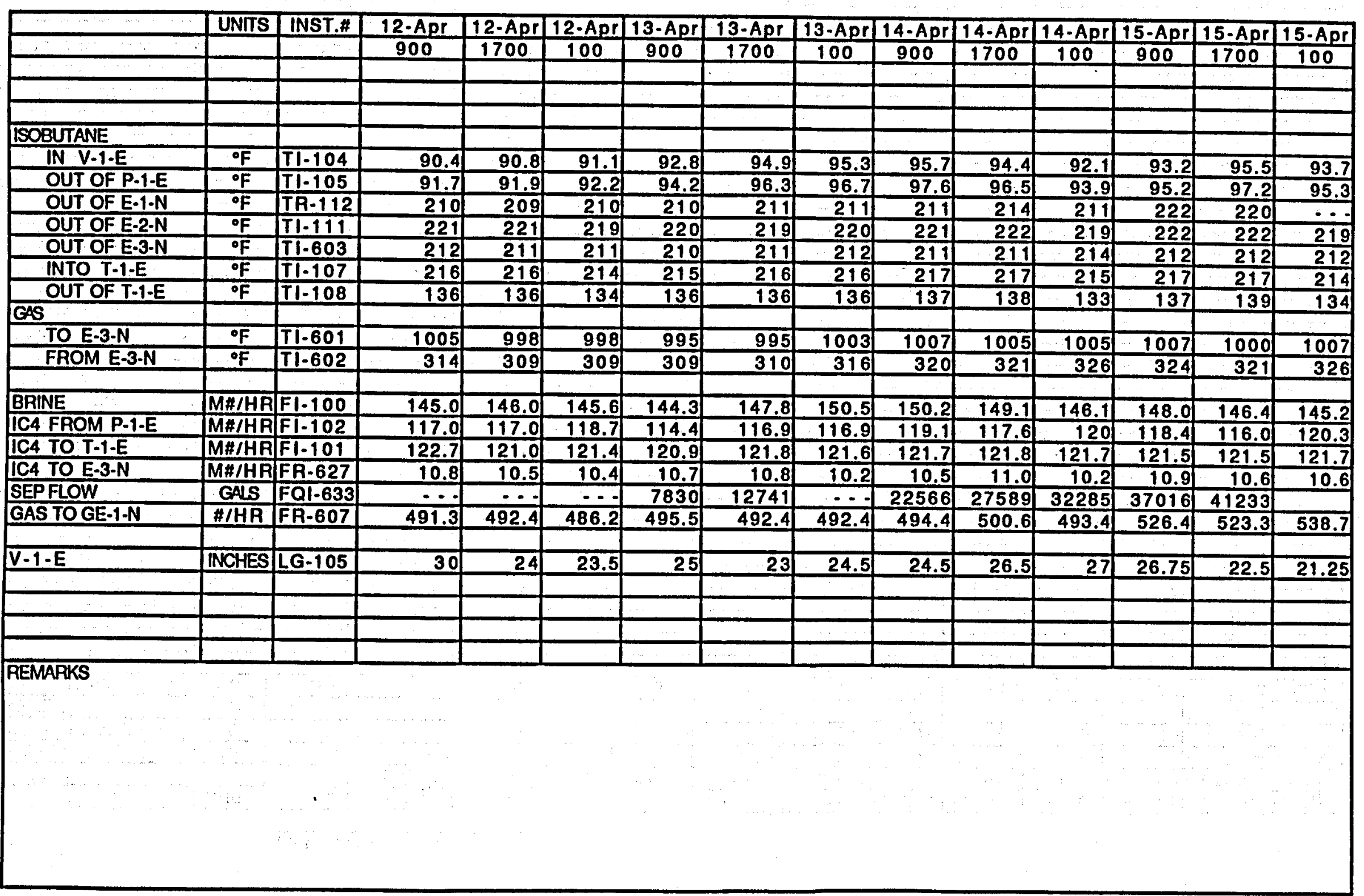


April 16-18, 1990

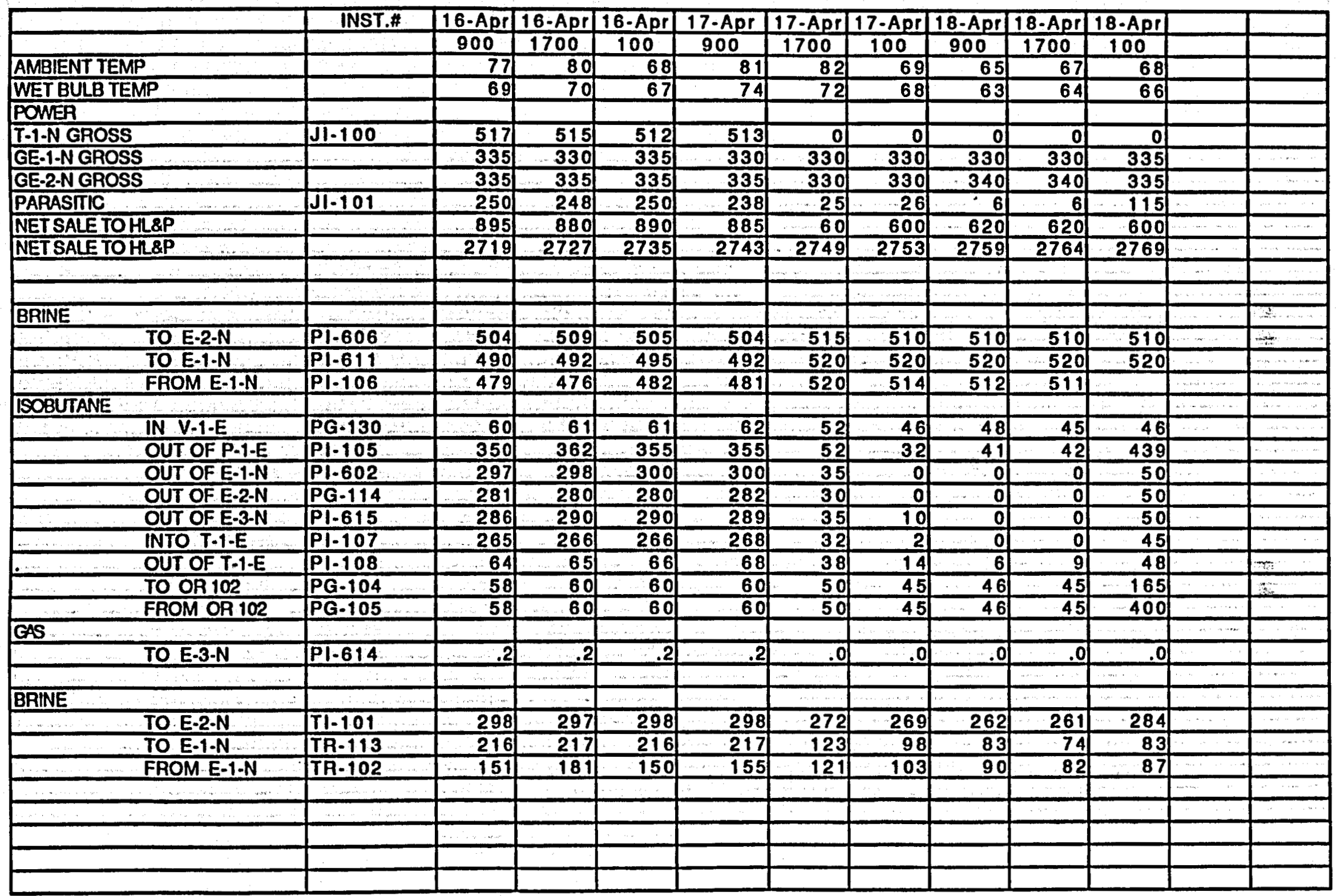


April 16-18, 1990

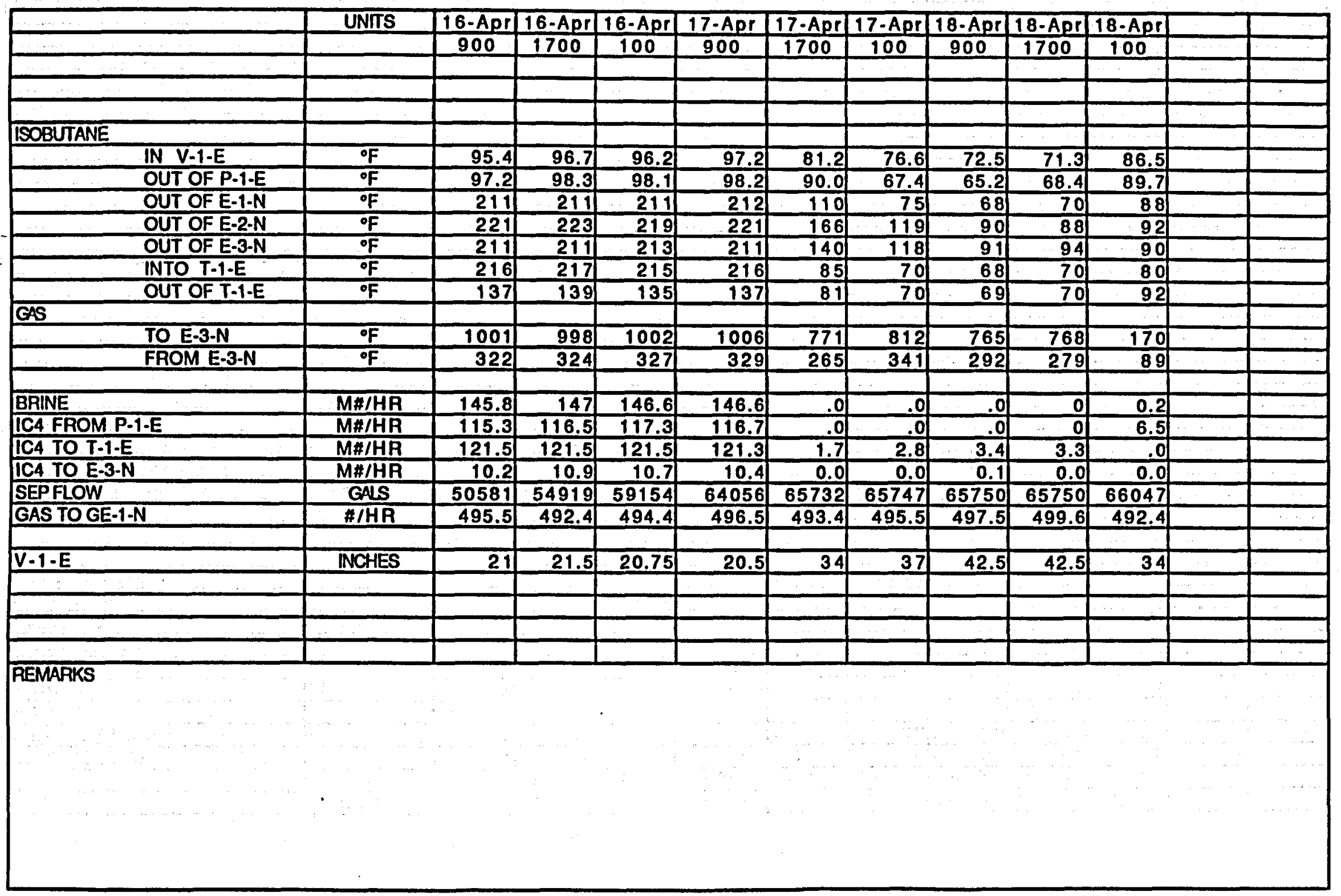


April 19-22, 1990

\begin{tabular}{|c|c|c|c|c|c|c|c|c|c|c|c|c|c|c|}
\hline & UNTS & INST.\# & 19-Apr & 19-Apr & 19-Apr & 20-Apr & 20.Apr & 20-Apr & 21-Apr & 21-Apr 2 & 21-Apr & 22-Apr & 22-Apr & 22-Apr \\
\hline & & & 900 & 1700 & 100 & 900 & 1700 & 100 & 900 & 1700 & 100 & 900 & 1700 & 100 \\
\hline AMBIENT TEMP & of & & 76 & 79 & 71 & 75 & 83 & 72 & 76 & 87 & 72 & 77 & 82 & 70 \\
\hline WET BULB TEMP & of & & 72 & 73 & 70 & 72 & 76 & 70 & 73 & 77 & 70 & 73 & 75 & 69 \\
\hline POWEA & & & & & & & & & & & & & & \\
\hline$T-1-N$ GROSS & $\overline{\mathrm{KW}}$ & J1-100 & 490 & 492 & 494 & 492 & 490 & 495 & 499 & 494 & 499 & 493 & 492 & 501 \\
\hline GE-1-NGROSS & KW & & 330 & 335 & 340 & 540 & 340 & 340 & 335 & 330 & 335 & 335 & 335 & 335 \\
\hline GE-2-N GROSS & $\overline{K W}$ & & 340 & 340 & 340 & 340 & 340 & 340 & 340 & 340 & 340 & 340 & 335 & 335 \\
\hline PARASITIC & $\mathbf{K W}$ & J1.101 & 239 & 251 & 250 & 250 & 249 & 240 & 250 & 249 & 250 & 239 & 248 & 250 \\
\hline NET SALE TOHL\&P & KW & & 890 & 875 & 890 & 880 & 875 & 890 & 880 & 860 & 880 & 870 & 880 & 870 \\
\hline NET SALE TOHL\&P & FDG & & 2774 & 2782 & 2790 & 2798 & 2805 & 2813 & 2821 & 2828 & 2836 & 2844 & 2852 & 2860 \\
\hline & KW-HR & & & & & & & & & & & 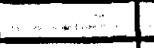 & $\cdots$ & \\
\hline & & & & & & & & & & & & & & \\
\hline BAINE & & & & & & & & & & & & & & \\
\hline TO E-2-N & PSIG & $P \mid-606$ & 500 & 504 & 505 & 505 & 506 & 509 & 505 & 503 & 505 & 505 & 506 & 505 \\
\hline TO E-1.N & PSIG & $P \mid-611$ & 490 & 490 & 490 & 490 & 491 & 493 & 490 & 489 & 495 & 490 & 490 & 495 \\
\hline FROM E-1-N & PSIG & $P 1.106$ & 480 & 479 & 482 & 482 & 477 & 481 & 481 & 476 & 481 & 481 & 479 & 481 \\
\hline ISOSUTANE & & & & & & & & & & & & & & \\
\hline IN V.1.E & PSIG & PG-130 & 63 & 65 & 64 & 64 & 66 & 63 & 64 & 64 & 63 & 64 & 67 & 62 \\
\hline OUT OF P.1-E & PSIG & P1.105 & 360 & 358 & 362 & 366 & 351 & 358 & 359 & 365 & 376 & 365 & 363 & 345 \\
\hline OUT OF E-1-N & PSIG & $\mathrm{P} \mid-602$ & 305 & 305 & 305 & 305 & 305 & 305 & 310 & 308 & 310 & 310 & 309 & 310 \\
\hline OUT OF E-2-N & PSIG & PG-114 & 275 & 278 & 280 & 280 & 278 & 280 & 280 & 280 & 280 & 280 & 280 & 280 \\
\hline OUT OF E-3-N & PSIG & $\mathrm{P} 1.615$ & 285 & 282 & 288 & 285 & 284 & 285 & 290 & 287 & 290 & 290 & 287 & 290 \\
\hline INTO T.I-E & PSIG & $P \mid=107$ & 263 & 263 & 263 & 263 & 264 & 264 & 265 & 264 & 265 & 265 & 265 & 265 \\
\hline OUT OF T-1-E & PSIG & $\mathrm{PI} 1.108$ & 67 & 68 & 68 & 69 & 69 & 69 & 68 & 69 & 65 & 363 & 63 & 63 \\
\hline TO OR 102 & PSIG & PG-104 & 65 & 63 & 60 & 65 & 66 & 65 & 65 & 64 & 65 & 65 & 65 & 65 \\
\hline FROM OR 102 & PSIG & PG.105 & 65 & 63 & 60 & 65 & 65 & 65 & 65 & 64 & 65 & 65 & 65 & 65 \\
\hline GAS & & & & & & & & & & & & & & \\
\hline TO E-3-N & PSIG & $\overline{P I-614}$ & .2 & .2 & .2 & .2 & .2 & .2 & .2 & .2 & .2 & .2 & 2 & .2 \\
\hline & & & & & & & & & & & & & & \\
\hline BAINE & & & & & & & & & & & & & & \\
\hline TO E-2-N & ${ }^{\circ} \mathrm{F}$ & TI-101 & 297 & 297 & 297 & 297 & 292 & 298 & 298 & 297 & 298 & 298 & 297 & 298 \\
\hline TO E-1-N & ${ }^{\circ} \mathrm{F}$ & TR.113 & 215 & 215 & 215 & 215 & 215 & 215 & 216 & 216 & 216 & 216 & 216 & 216 \\
\hline FROM E-1-N & of & TR.102 & 149 & 149 & 149 & 158 & 149 & 207 & 192 & 149 & 224 & 149 & 149 & 149 \\
\hline & & & & & & & & & & & & & & \\
\hline & & & & & & & & & & & & & & \\
\hline & & & & & & & & & & & & & & \\
\hline & & & & & & & & & & & & & & \\
\hline & & & & & & & & & & & & & & \\
\hline
\end{tabular}




\section{GEOPRESSURED HYBRID POWER SYSTEM - MANAGEMENT REPORT DATA}

April 19-22, 1990

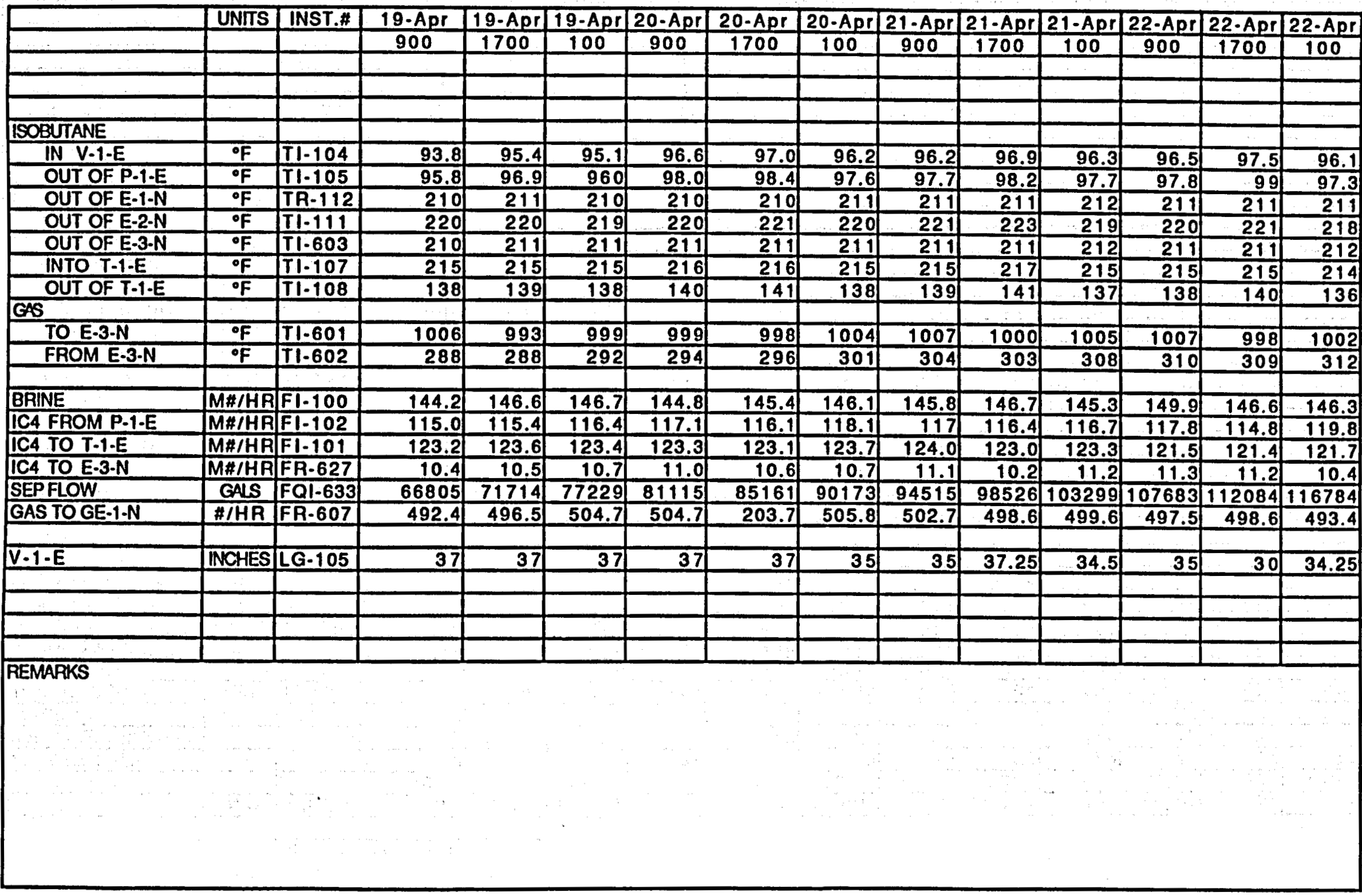


April 23-25, 1990

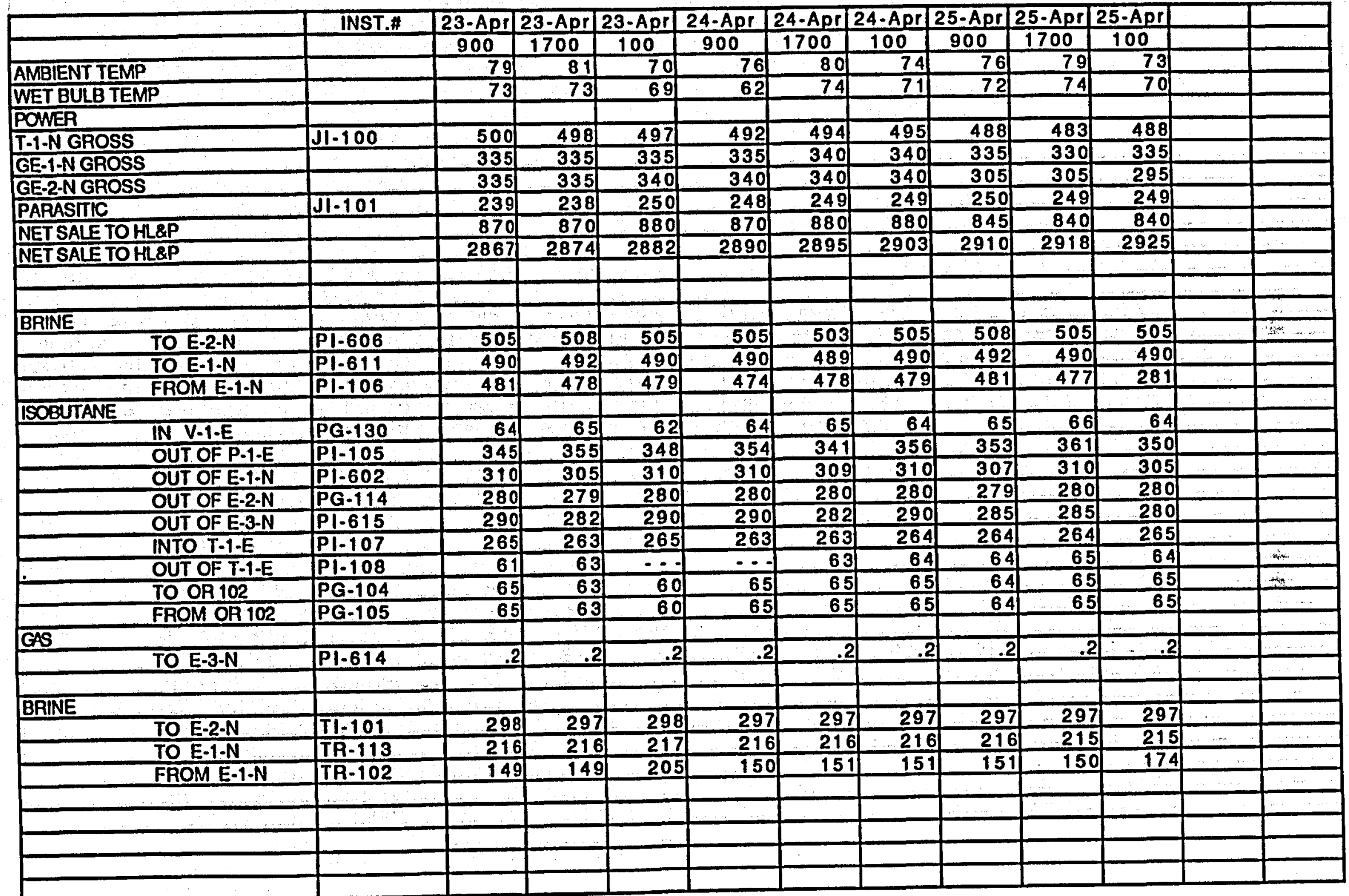


April 23.25, 1990

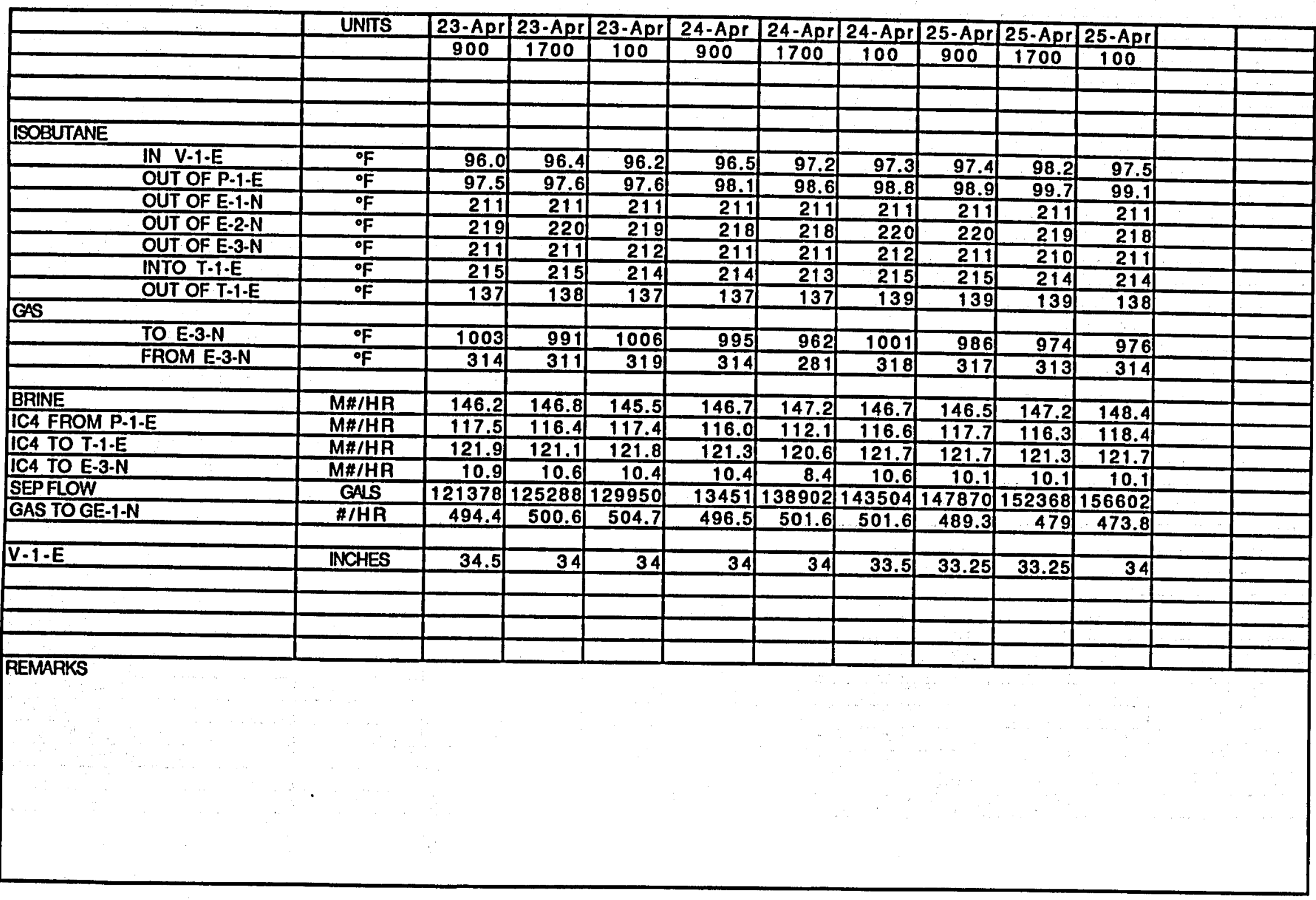


April 26-29, 1990

\begin{tabular}{|c|c|c|c|c|c|c|c|c|c|c|c|c|c|c|}
\hline & UNITS & INST.\# & 26-Apr & 26-Apr & 26-Apr & 27-Apr & 27-Apr & 27-Ape: & 28-Apr & 28-Apr & 28-Apr & 29-Apr & 29.Apr & 29-Apr \\
\hline & & & 900 & 1700 & 100 & 900 & 1700 & \begin{tabular}{|l|}
100 \\
\end{tabular} & 900 & 1700 & 100 & 900 & 1700 & 100 \\
\hline AMBIENT TEMP & $\circ \mathrm{F}$ & & 76 & 62 & 68 & 73 & 82 & 64 & 67 & 82 & 74 & 79 & 80 & 76 \\
\hline WET BULB TEMP & ${ }^{\circ} \mathrm{F}$ & & 72 & 62 & 65 & 69 & 76 & 63 & 60 & 76 & 71 & 77 & 76 & 62 \\
\hline \multicolumn{15}{|l|}{ POWEA } \\
\hline T-1.N GROSS & KW & $J 1-100$ & 494 & 525 & 515 & 504 & 476 & 533 & 533 & 493 & 493 & 497 & 479 & 491 \\
\hline GE-1-N GROSS & $\mathrm{KW}$ & & 335 & 335 & 340 & 340 & 335 & 0 & 0 & 0 & 0 & 0 & 0 & 0 \\
\hline GE-2-N GROSS & $\overline{K W}$ & & 315 & 305 & 320 & 280 & 0 & 340 & 345 & 340 & 330 & 340 & 330 & 335 \\
\hline PARASTIC & $\mathrm{KW}$ & J1.101 & 248 & 251 & 238 & 237 & 248 & 252 & 249 & 248 & 248 & 239 & 247 & 247 \\
\hline NET SALE TOHLAP & $\mathrm{KW}$ & & 855 & 870 & 880 & 835 & 560 & 600 & 615 & 580 & 580 & 585 & 570 & 560 \\
\hline \multirow{2}{*}{ NET SALE TOHLEP } & $F D G$ & & 2932 & 2940 & 2947 & 2957 & 2962 & 2968 & 2972 & 2978 & 2982 & 298 & 2992 & 2997 \\
\hline & $\mathrm{KW}-\mathrm{HA}$ & & & & & & & & & & & & & \\
\hline \multirow{2}{*}{\multicolumn{15}{|c|}{ BRINE }} \\
\hline & & & & & & & & & & & & & & \\
\hline TO E-2-N & PSIG & $\mathrm{Pl.606}$ & 508 & 505 & 505 & 505 & 505 & 505 & 500 & 505 & 505 & 503 & 508 & 505 \\
\hline TO E-1-N & PSIG & PI.611 & 492 & 490 & 492 & 492 & 490 & 490 & 488 & 470 & 490 & 490 & 490 & 490 \\
\hline FROM E-1-N & PSIG & $P 1.106$ & 481 & 478 & 479 & 480 & 478 & 477 & 480 & 475 & 477 & 476 & 477 & 477 \\
\hline ISOBUTANE & & & & & & & & & & & & & & \\
\hline IN $V-1 . E$ & PSIG & PG-130 & 65 & 62 & 62 & 63 & 65 & 58 & 57 & 64 & 64 & 66 & 67 & 66 \\
\hline OUT OF P.1.E & PSIG & PI.105 & 359 & 347 & 343 & 358 & 350 & 333 & 344 & 364 & 351 & 354 & 357) & 357 \\
\hline OUT OF E-1-N & PSIG & $\mathrm{PI} 1.602$ & 307 & 310 & 310 & 307 & 305 & 310 & 309 & 310 & 310 & 312 & 310 & 310 \\
\hline OUT OF E-2-N & PSIG & $\overline{P G-114}$ & 279 & 280 & 280 & 281 & 275 & 280 & 282 & 280 & 280 & 283 & 280 & 280 \\
\hline OUT OF E-3-N & PSIG & P1.615 & 285 & 285 & 290 & 287 & 282 & 290 & 289 & 285 & 290 & 289 & 290 & 290 \\
\hline INTO T.1-E & PSIG & $\mathrm{P} \mid-107$ & 264 & 266 & 267 & 264 & 259 & 270 & 264 & 265 & 265 & 265 & 265 & 265 \\
\hline OUT OF T-1-E & PSIG & $\mathrm{PI}-108$ & 64 & 61 & 62 & 62 & 64 & 62 & 68 & 64 & 65 & 65 & 66 & 66 \\
\hline TO OR 102 & PSIG & PG-104 & 63 & 65 & 60 & 61 & 65 & 60 & 52 & 65 & 65 & 62 & 65 & 65 \\
\hline FROM OR 102 & PSIG & PG-105 & 62 & 65 & 60 & 61 & 65 & 60 & 52 & 65 & 65 & 62 & 65 & 65 \\
\hline \multicolumn{15}{|l|}{ GAS } \\
\hline TO E-3.N & PSIG & $\mathrm{P} 1-614$ & .2 & .2 & .2 & .2 & .2 & .2 & .2 & .2 & .2 & .2 & .2 & .2 \\
\hline \multirow{2}{*}{\multicolumn{15}{|c|}{ BRINE }} \\
\hline & & & & & & & & & & & & & & \\
\hline TO E-2-N & of & $\mathrm{TI}-101$ & 297 & 297 & 297 & 297 & 297 & 297 & 298 & 297 & 297 & 297 & 297 & 297 \\
\hline TO E-1-N & of & TA-113 & 216 & 217 & 218 & 216 & 214 & 216 & 218 & 217 & 217 & 217 & 216 & 216 \\
\hline FAOM E-1-N & $\circ \mathrm{F}$ & TR-102 & 151 & 149 & 150 & 151 & 151 & 151 & 149 & 152 & 154 & 153 & 153 & 153 \\
\hline & & & & & & & & & & & & & & \\
\hline & & & & & & & & & & & & & & \\
\hline & & & & & & & & & & & & & & \\
\hline & & & & & & & & & & & & & & \\
\hline & & & & & & & & & & & & & & \\
\hline
\end{tabular}


GEOPRESSURED HYBRID POWER SYSTEM - MANAGEMENT REPORT DATA

April $26-29, \quad 1990$

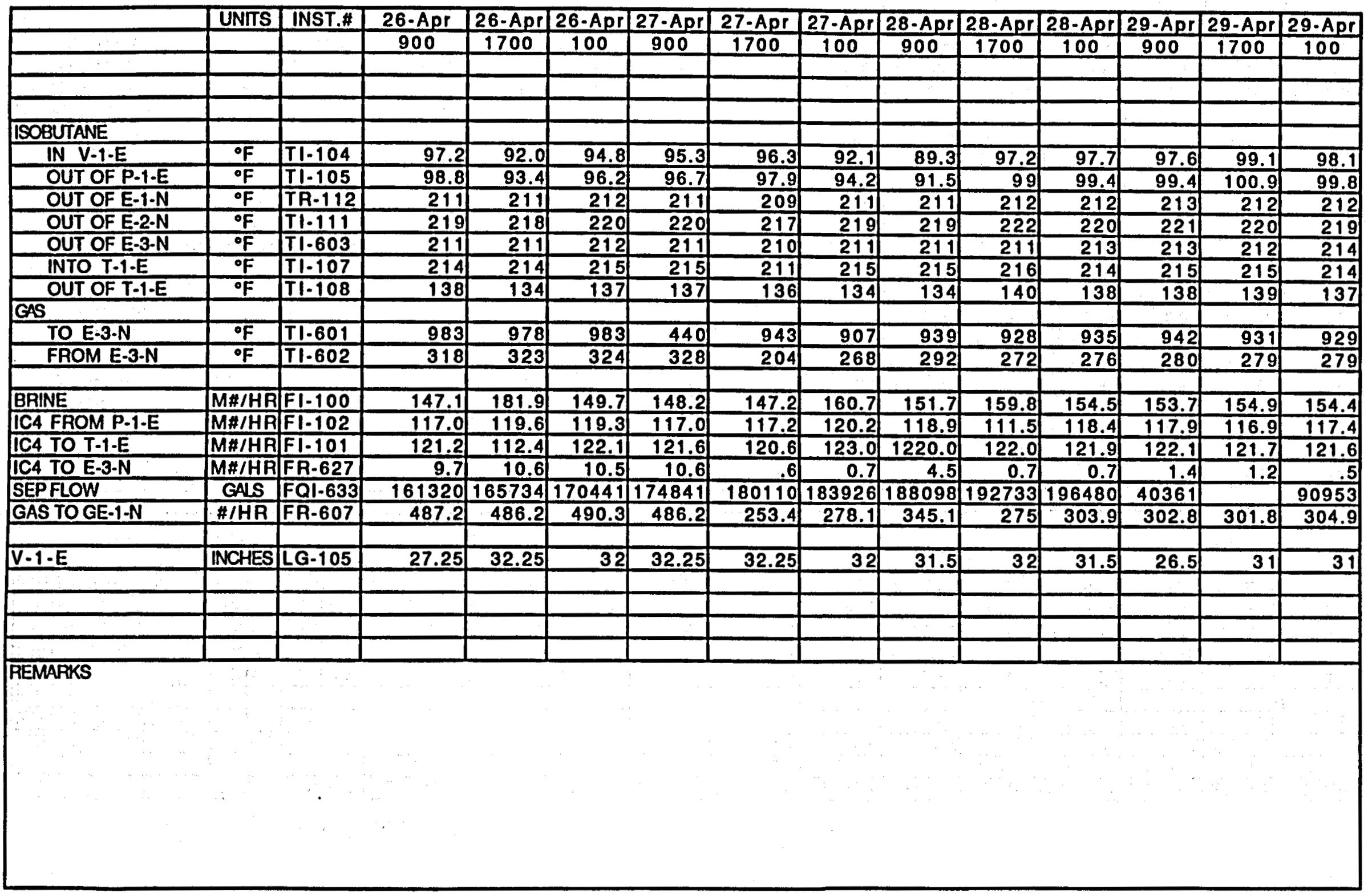


April 30-May 2, 1990

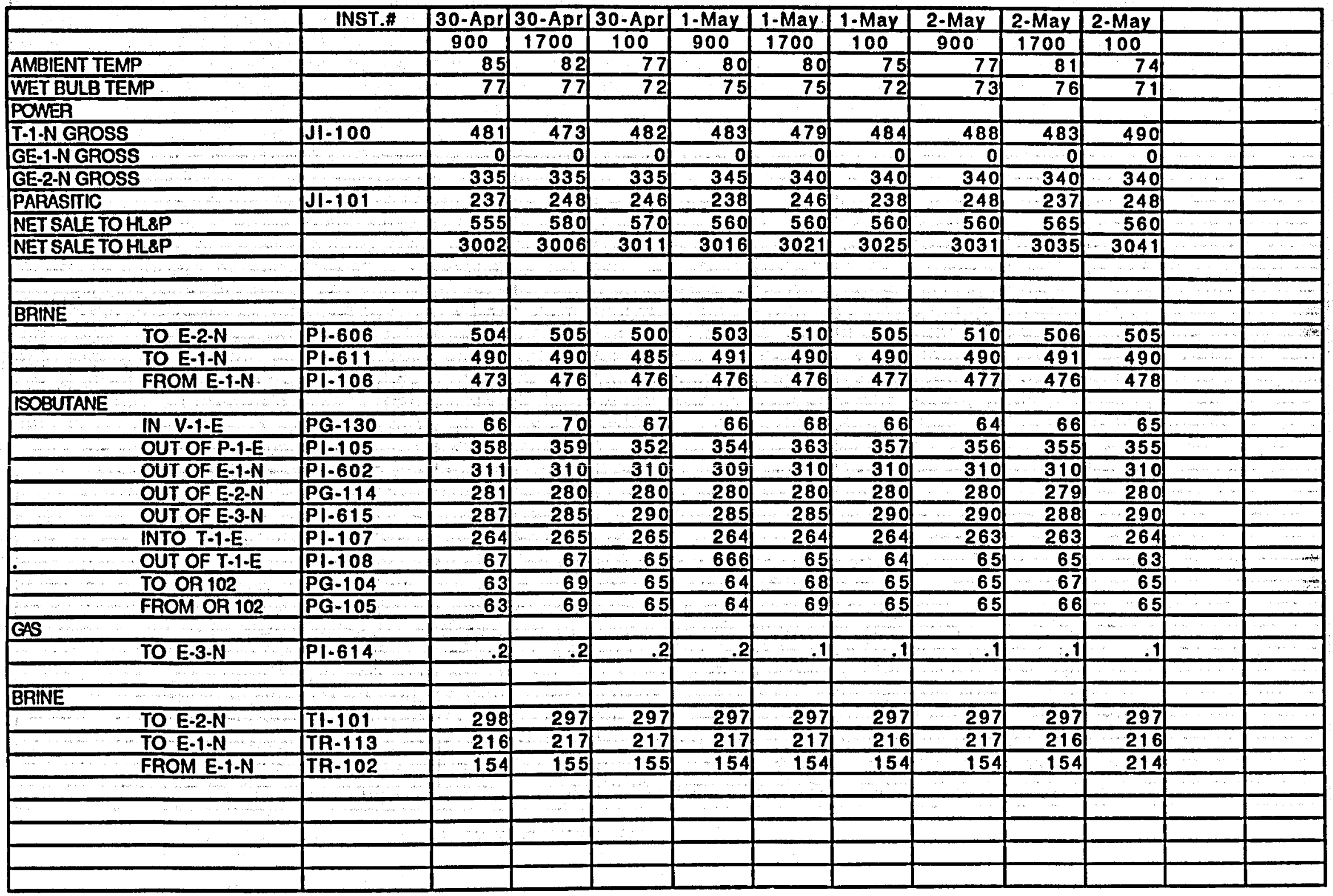


April 30-May 2, 1990

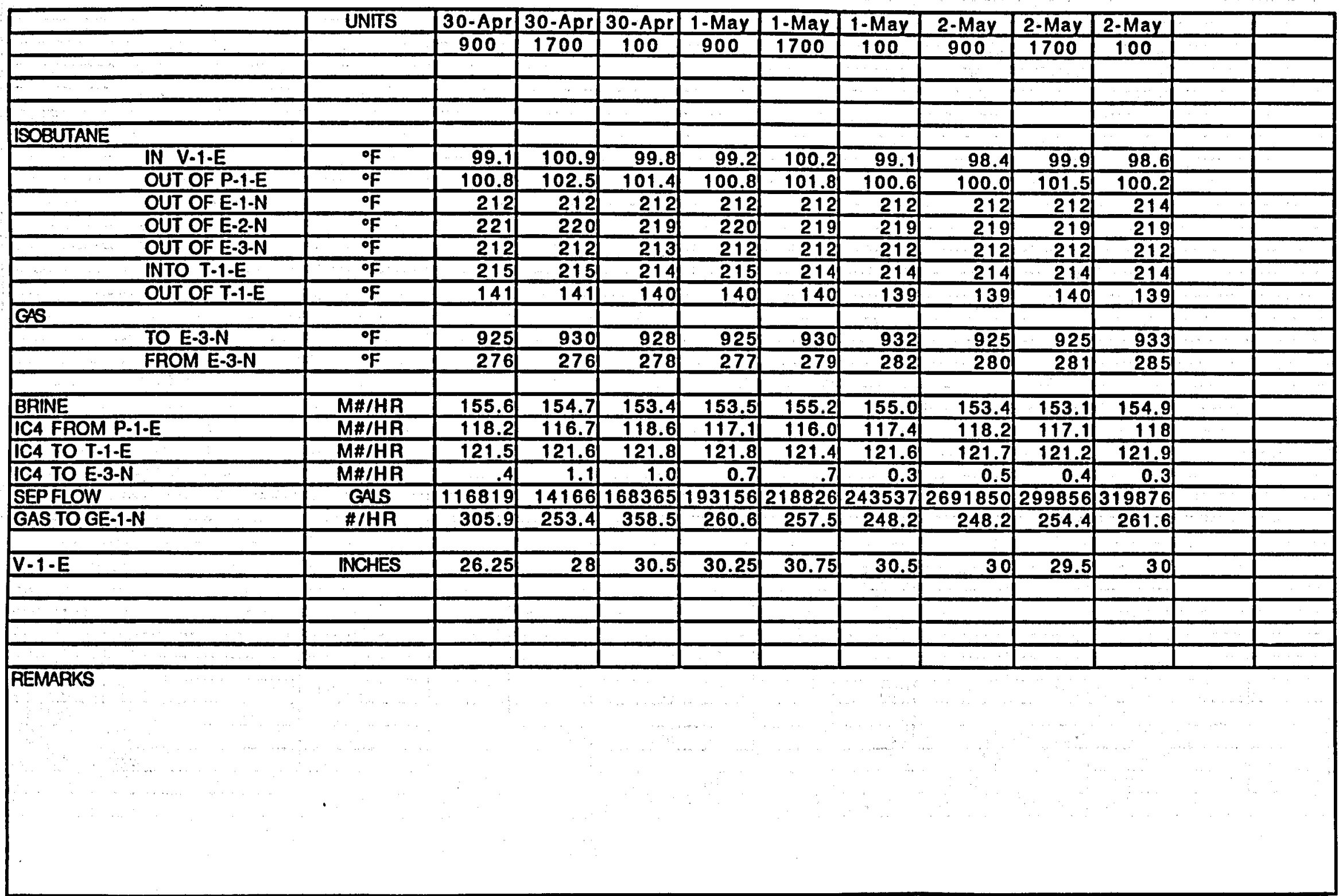


May 3-6, 1990

\begin{tabular}{|c|c|c|c|c|c|c|c|c|c|c|c|c|c|c|}
\hline & UNITS & INST.\# & 3-May & 3.May & 3.May & 4-May & 4-May & 4-May & 5-May & 5-May & 5.May & 6-May & 6-May & 6-May \\
\hline & & & 900 & 1700 & 100 & 900 & 1700 & 100 & 900 & 1700 & 100 & 900 & 1700 & 100 \\
\hline AMBIENT TEMP & ${ }^{\circ} \mathrm{F}$ & & 77 & 75 & 68 & 71 & 73 & 68 & 61 & 67 & 64 & 70 & 76 & 64 \\
\hline WET BULB TEMP & of & & 74 & 75 & 68 & 67 & 65 & 61 & 53 & 62 & 61 & 60 & 61 & 59 \\
\hline \multicolumn{15}{|l|}{ POWER } \\
\hline T-1-N GROSS & KW & $J 1-100$ & 485 & 484 & 499 & 501 & 503 & 535 & 545 & 533 & 535 & 538 & 523 & 531 \\
\hline GE-1-N GROSS & $\mathrm{KW}$ & & 0 & 0 & 0 & of & 0 & 320 & 320 & 320 & 320 & 310 & 300 & 310 \\
\hline GE-2-N GROSS & $\overline{\mathrm{KW}}$ & & 340 & 340 & 340 & 340 & 345 & 340 & 340 & 340 & 340 & 340 & 340 & 340 \\
\hline PARASITIC & $\overline{K W}$ & J1-101 & 238 & 238 & 249 & 246 & 250 & 253 & 252 & 250 & 253 & 253 & 251 & 252 \\
\hline NET SALE TOHL\&P & KW & & 560 & 570 & 580 & 580 & 600 & 905 & 900 & 905 & 905 & 890 & 880 & 900 \\
\hline \multirow[t]{2}{*}{ NET SALE TO HL\&P } & $\mathbf{F D G}$ & & 3045 & 3050 & 3055 & 3060 & 3065 & 3073 & 3081 & 3089 & 3097 & 3105 & 3111 & 3119 \\
\hline & KW-HR & & & & & & & & & & & & & \\
\hline & 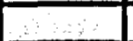 & & & & & & & & & & & & & \\
\hline \multicolumn{15}{|l|}{ BRINE } \\
\hline TO E-2-N & PSIG & PI.606 & 505 & 505 & 505 & 505 & 510 & 505 & 505 & 510 & 505 & 505 & 510 & 505 \\
\hline TO E-1-N & PSIG & PI-611 & 490 & 490 & 490 & 495 & 495 & 490 & 490 & 495 & 495 & 490 & 495 & 495 \\
\hline FROM E-1-N & PSIG & $\mathrm{PI} .106$ & 477 & 478 & 479 & 475 & 478 & 478 & 481 & 475 & 481 & 480 & 476 & 480 \\
\hline ISOBUTANE & & & & & & & & & & & & & & \\
\hline IN V-1.E & PSIG & PG-130 & 65 & 65 & 64 & 62 & 62 & 58 & 55 & 58 & 58 & 55 & 58 & 57 \\
\hline OUT OF P-1-E & PSIG & PI.105 & 360 & 363 & 341 & 339 & 352 & 339 & 348 & 349 & 340 & 342 & 354 & 340 \\
\hline OUT OF E-1-N & PSIG & PI-602 & 310 & 310 & 310 & 310 & 310 & 310 & 305 & 310 & 310 & 310 & 307 & 310 \\
\hline OUT OF E-2-N & PSIG & $P G-114$ & 280 & 278 & 280 & 280 & 280 & 280 & 280 & 280 & 280 & 280 & 280 & 280 \\
\hline OUT OF E-3-N & PSIG & $P \mid-615$ & 290 & 285 & 290 & 290 & 285 & 290 & 290 & 290 & 290 & 290 & 285 & 290 \\
\hline INTO T-I.E & PSIG & PI-107 & 264 & 263 & 265 & 265 & 265 & 267 & 263 & 266 & 268 & 267 & 264 & 263 \\
\hline OUT OF T-1-E & PSIG & PI.108 & 64 & 62 & 59 & 58 & 56 & 54 & 51 & 56 & 54 & 53 & 51 & 50 \\
\hline TO OA 102 & PSIG & PG-104 & 65 & 65 & 65 & 65 & 60 & 60 & 55 & 55 & 55 & 55 & 55 & 60 \\
\hline FROM OA 102 & PSIG & PG.105 & 56 & 65 & 65 & 65 & 60 & 60 & 55 & 55 & 55 & 55 & 55 & 60 \\
\hline \multicolumn{15}{|l|}{ GAS } \\
\hline TO E-3-N & PSIG & $P 1.614$ & .2 & .2 & .1 & .1 & .1 & .2 & 2 & .2 & .2 & 2 & .2 & .2 \\
\hline & & & & & & & & & & & & & & \\
\hline \multicolumn{15}{|l|}{ BRINE } \\
\hline TO E-2-N & ${ }^{\circ} \mathrm{F}$ & $T 1.101$ & 297 & 297 & 298 & 298 & 298 & 298 & 297 & 298 & 298 & 298 & 298 & 298 \\
\hline TO E-1-N & ${ }^{\circ} \mathrm{F}$ & TA-113 & 217 & 217 & 218 & 216 & 214 & 217 & 217 & 217 & 217 & 219 & 217 & 217 \\
\hline FROM E-1.N & o $F$ & TA-102 & 155 & 158 & 153 & 153 & 152 & 149 & 148 & 149 & 149 & 149 & 148 & 148 \\
\hline & & & & & & & & $\therefore$ & & & & & & \\
\hline & & & & & & & & & & & & & & \\
\hline & & & & & & & & & & & & & & \\
\hline & & & & & & & & & & & & & & \\
\hline & & & & & & & & & & & & & & \\
\hline
\end{tabular}


GEOPRESSURED HYBRID POWER SYSTEM - MANAGEMENT REPORT DATA

May 3-6, 1990

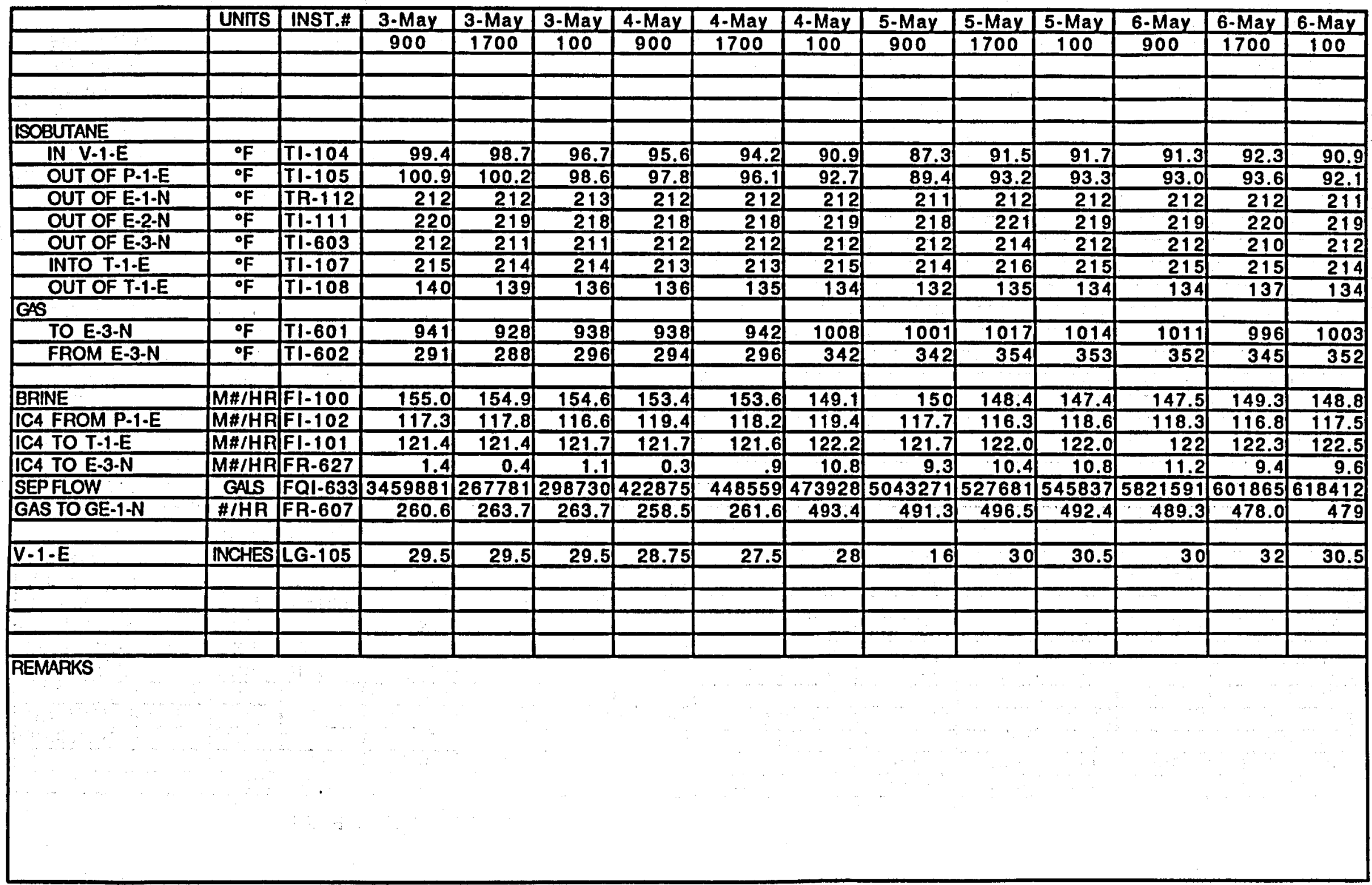


May 7.9, 1990

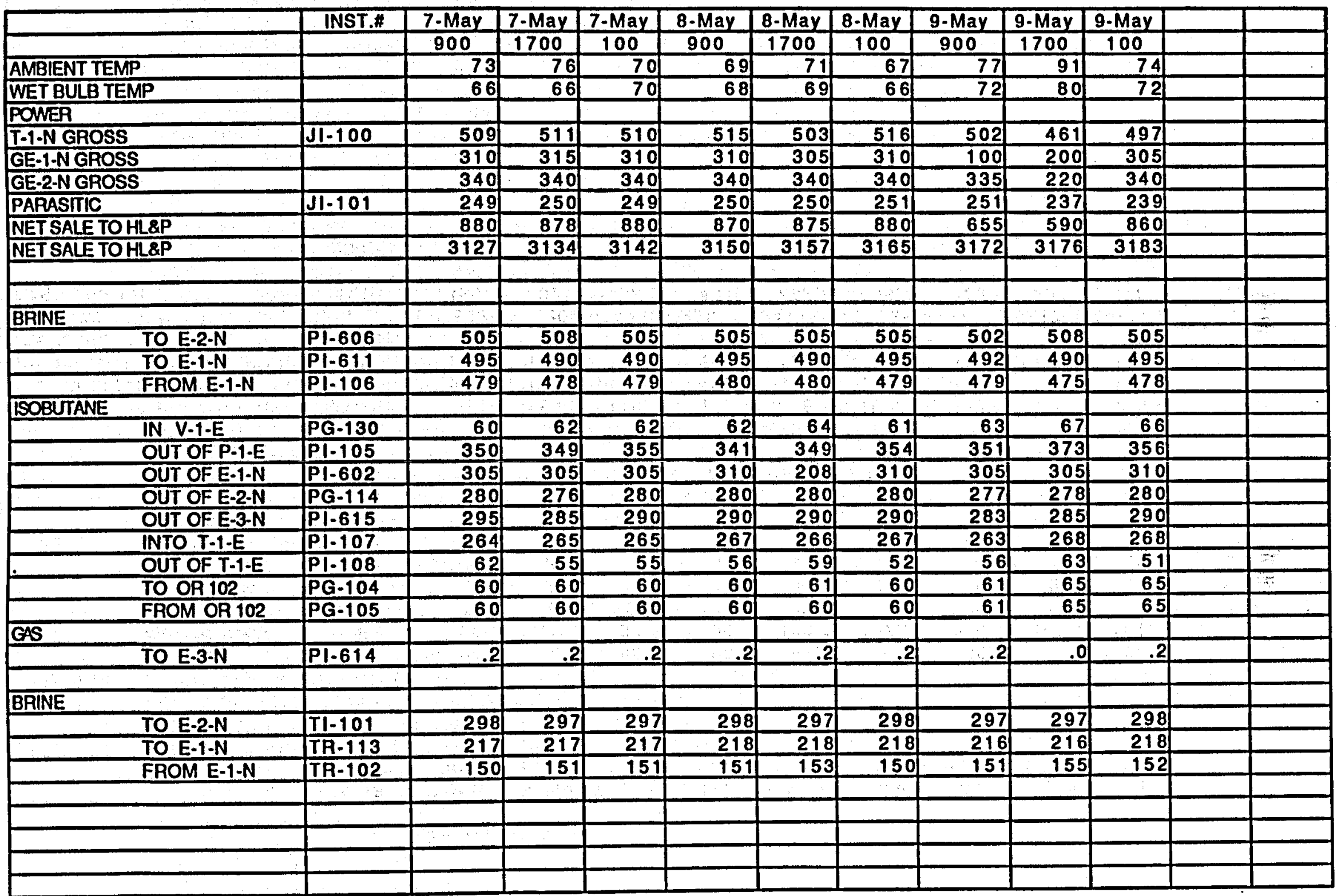


May 7-9, 1990

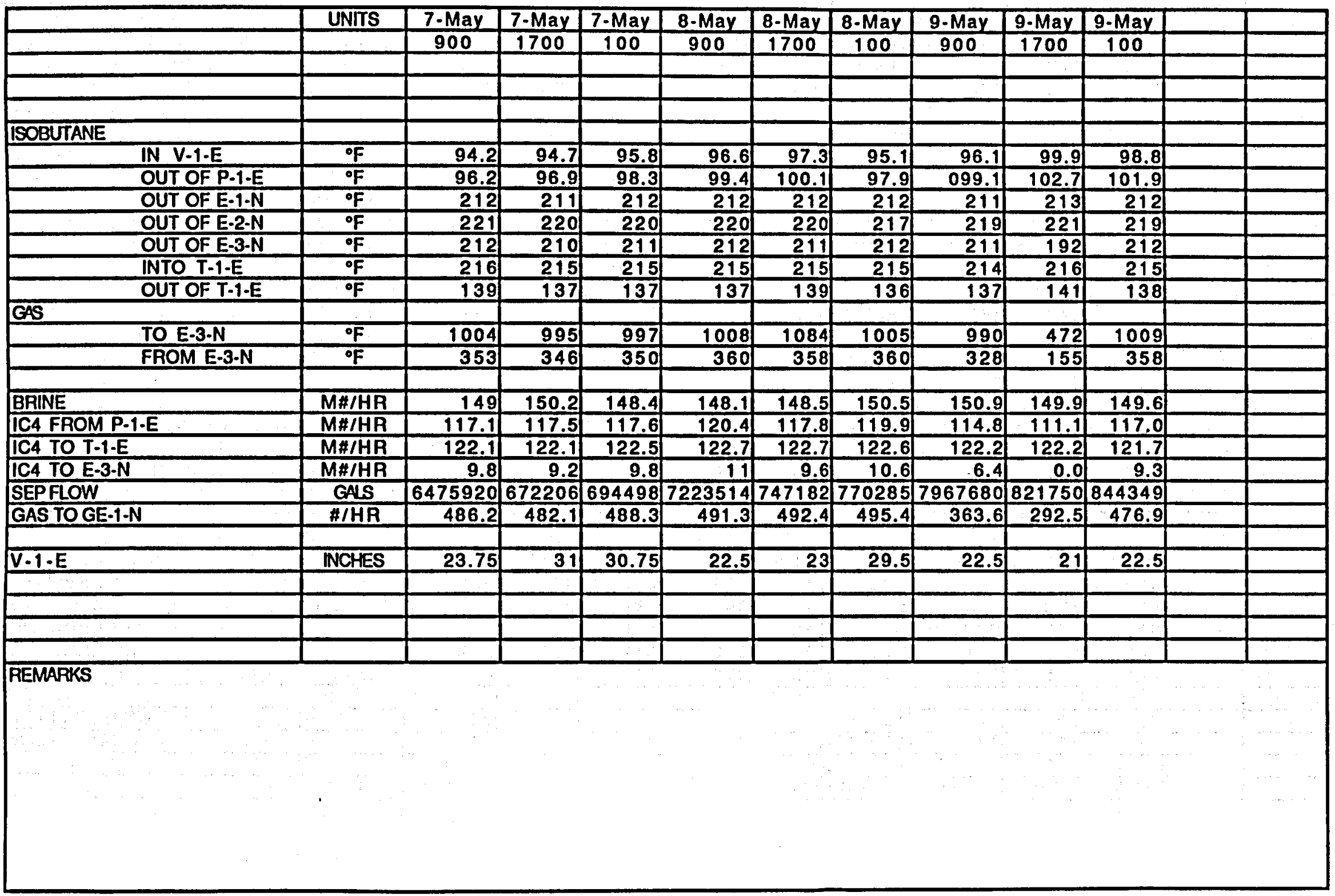


May $10-13,1990$

\begin{tabular}{|c|c|c|c|c|c|c|c|c|c|c|c|c|c|c|}
\hline & UNITS & INST.\# & 10.May & 10-May & 10-May & 11-May & 11-May & 11-May & 12-May & 12-May & 12-May & 13.May & 13-May & 13-May \\
\hline & & & 900 & 1700 & 100 & 900 & 1700 & 100 & 900 & 1700 & 100 & 900 & 1700 & 100 \\
\hline AMBIENT TEMP & of & & 69 & 80 & 70 & 79 & 80 & 76 & 77 & 82 & 77 & 77 & 88 & 77 \\
\hline WET BULB TEMP & OF & & 59 & 66 & 68 & 76 & 75 & 74 & 74 & 76 & 75 & 76 & 79 & 75 \\
\hline \multicolumn{15}{|l|}{ POWEA } \\
\hline T-1-N GROSS & $\overline{K W}$ & $J 1.100$ & 527 & 526 & 517 & 4911 & 4911 & 486 & 490 & 484 & 486 & 495 & 482 & 486 \\
\hline GE-1-N GROSS & $\mathrm{KW}$ & & 325 & 325 & 325 & 320 & 320 & 320 & 320 & 310 & 300 & 316 & 315 & 300 \\
\hline GE-2-N GROSS & $\mathrm{KW}$ & & 345 & 345 & 340 & 345 & 335 & 340 & 335 & 320 & 320 & 325 & 320 & 330 \\
\hline PARASITIC & $\overline{\mathrm{KW}}$ & $J 1-101$ & 251 & 249 & 250 & 250 & 249 & 238 & 249 & 239 & 248 & 238 & 250 & 250 \\
\hline NET SALE TO HL\&P & $\mathrm{KW}$ & & 905 & 900 & 900 & 860 & 850 & 860 & 845 & 820 & 810 & 840 & 840 & 830 \\
\hline \multirow{3}{*}{ NET SALE TO HL\&P } & $\overline{A D G}$ & & 3191 & 3198 & 3207 & 3214 & 3222 & 3230 & 3237 & 3244 & 3251 & 3259 & 3266 & 3272 \\
\hline & $\mathrm{KW}-\mathrm{HR}$ & & & & & & & & & & & & & \\
\hline & + & & & & & & & & & & & & & \\
\hline \multicolumn{15}{|l|}{ BAINE } \\
\hline TO E-2.N & PSIG & $P \mid-606$ & 503 & 510 & 505 & 502 & 505 & 505 & 505 & 505 & 510 & 507 & 510 & 510 \\
\hline TOE-1-N & PSIG & $\overline{P I .611}$ & 492 & 490 & 490 & 489 & 490 & 490 & 492 & 490 & 495 & 494 & 490 & 490 \\
\hline FROM E-1-N & PSIG & $P 1.106$ & 480 & 475 & 480 & 479 & 477 & 477 & 476 & 478 & 480 & 479 & 480 & 479 \\
\hline \multicolumn{15}{|l|}{ ISOBUTANE } \\
\hline IN $V-1-E$ & PSIG & PG.130 & 57 & 58 & 60 & 65 & 65 & 66 & 66 & 68 & 68 & 67 & 69 & 68 \\
\hline OUT OF P-1-E & PSIG & $P \mid-105$ & 346 & 348 & 348 & 347 & 355 & 359 & 358 & 358 & 356 & 347 & 361 & 359 \\
\hline OUT OF E-1-N & PSIG & $\mathrm{PI} .602$ & 306 & 307 & 310 & 309 & 310 & 308 & 309 & 310 & 312 & 311 & 310 & 310 \\
\hline OUT OF E-2-N & PSIG & PG.114 & 279 & 280 & 280 & 281 & 280 & 280 & 280 & 280 & 283 & 284 & 284 & 280 \\
\hline OUT OF E-3-N & PSIG & P1-615 & 284 & 289 & 290 & 287 & 290 & 285 & 285 & 290 & 290 & 291 & 290 & 290 \\
\hline INTO T-1-E & PSIG & $P 1-107$ & 263 & 266 & 265 & 265 & 266 & 265 & 265 & 261 & 268 & 270 & 268 & 267 \\
\hline OUT OF T-1-E & PSIG & $P \mid-108$ & 52 & 55 & 53 & 59 & 59 & 58 & 68 & 60 & 61 & 63 & 62 & 59 \\
\hline TO OA 102 & PSIG & PG-104 & 52 & 60 & 60 & 64 & 65 & 65 & 65 & 68 & 68 & 68 & -69 & 69 \\
\hline FROM OA 102 & PSIG & PG.105 & 53 & 60 & 60 & 347 & 65 & 65 & 65 & 68 & 68 & 68 & 69 & 69 \\
\hline \multicolumn{15}{|l|}{ GAS } \\
\hline TO E-3-N & PSIG & $\mathrm{PI} \cdot 614$ & .2 & .2 & .2 & .3 & .2 & .2 & .3 & .3 & .2 & .3 & 2 & .3 \\
\hline \multirow{2}{*}{\multicolumn{15}{|c|}{ BRINE }} \\
\hline & & & & & & & & & & & & & & \\
\hline TO E-2-N & ${ }^{\circ} \mathrm{F}$ & $T 1-101$ & 297 & 297 & 297 & 297 & 297 & 297 & 297 & 297 & 297 & 298 & 297 & 297 \\
\hline TOE-1-N & of & TR-113 & 217 & 219 & 218 & 219 & 219 & 219 & 219 & 219 & 219 & 220 & 219 & 218 \\
\hline FROM E-1-N & ${ }^{\circ} \mathrm{F}$ & TR-102 & 149 & 151 & 152 & 155 & 155 & 155 & 155 & 157 & 155 & 155 & 181 & 156 \\
\hline & & & & & & & & & & & & & & \\
\hline & & & & & & & & & & & & & & \\
\hline & & & & & & & & & & & & & & \\
\hline & & & & & & & & & & & & & & \\
\hline & & & & & & & & & & & & & & \\
\hline
\end{tabular}


GEOPRESSURED HYBRID POWER SYSTEM - MANAGEMENT REPORT DATA

May 10-13, 1990

\begin{tabular}{|c|c|c|c|c|c|c|c|c|c|c|c|c|c|c|}
\hline & UNITS & INST.\# & 10-May & 10. May & 10-May & 11-May & 11-May & 11-May & 12-May & 12-May & 12-May & 13-May & 13-May & 13-May \\
\hline & & & 900 & 1700 & 100 & 900 & 1700 & 100 & 900 & 1700 & 100 & 900 & 1700 & 100 \\
\hline & & & & & & & & & & & & & & \\
\hline & & & & & & & & & & & & & & \\
\hline & & & & & & & & & & & & & & \\
\hline \multicolumn{15}{|l|}{ ISOBUTANE } \\
\hline IN V.1.E & ${ }^{\circ} \mathrm{F}$ & $T 1.104$ & 91.1 & 93.5 & 94.8 & 99.4 & 100.3 & 100.4 & 87.3 & 101.6 & 101.6 & 101.2 & 103.3 & 101.5 \\
\hline OUT OF P.1.E & ${ }^{\circ} \mathrm{F}$ & TI.105 & 93.9 & 95.4 & 97.3 & 102.3 & 103.1 & 103.4 & 89.4 & 104.7 & 104.5 & 104.1 & 104.9 & 104.6 \\
\hline OUT OF E-1-N & •F & TR-112 & 212 & 213 & 213 & 213 & 213 & 210 & 211 & 214 & 214 & 214 & 214 & 214 \\
\hline OUT OF E-2-N & of & Tl.111 & 220 & 221 & 220 & 222 & 221 & 221 & 218 & 222 & 222 & 223 & 223 & 222 \\
\hline OUT OF E-3-N & of & TI-603 & 211 & 211 & 212 & 212 & 211 & 211 & 212 & 213 & 214 & 213 & 212 & 212 \\
\hline INTO T-1-E & ${ }^{\circ} \mathrm{F}$ & TI.107 & 215 & 218 & 216 & 217 & 216 & 216 & 214 & 216 & 216 & 217 & 217 & 216 \\
\hline OUT OF T-1-E & ${ }^{\circ} \mathrm{F}$ & $\mathrm{TI}-108$ & 136 & 139 & 138 & 142 & 142 & 141 & 132 & 0 & 142 & 143 & 144 & 142 \\
\hline \multicolumn{15}{|l|}{ GAS } \\
\hline TO E-3-N & \% & $T 1.601$ & 1004 & 998 & 1003 & 998 & 994 & 990 & 1001 & 1001 & 993 & 999 & 996 & 1000 \\
\hline FROM E-3-N & of & $\mathrm{TI}-602$ & 356 & 355 & 356 & 355 & 355 & 356 & 342 & 367 & 367 & 368 & 364 & 366 \\
\hline BRINE & M\#/HR & FI.100 & 148.1 & 150.4 & 152.2 & 151.3 & 151.7 & 1530.0 & & & & & & \\
\hline ICAIFE FOM P-1-E & $\frac{\mathrm{M} \# / \mathrm{HA}}{\mathrm{M} \# / \mathrm{HA}}$ & $\frac{1-1.100}{F 1.102}$ & $\frac{148.1}{116.2}$ & $\frac{150.4}{115.4}$ & $\frac{152.2}{116.1}$ & $\frac{151.3}{118.1}$ & $\frac{151.7}{117.3}$ & $\frac{1530.0}{117.4}$ & $\begin{array}{r}150 \\
117.7\end{array}$ & $\frac{152.4}{118.3}$ & $\frac{150.3}{18.4}$ & $\frac{152.9}{118.9}$ & $\frac{151.1}{115.6}$ & $\frac{153}{1167}$ \\
\hline IC4 TO T-1-E & $\overline{M \# / H R}$ & $F \mid-101$ & 121.3 & 121.8 & 121.7 & 121.7 & 121.8 & 121.5 & 121.7 & 121.4 & $\frac{10.4}{121.8}$ & $\frac{110.9}{122.6}$ & $\frac{115.6}{121.8}$ & $\frac{116.8}{122.0}$ \\
\hline IC4 TO E-3-N & $\overline{M \# / H R}$ & FR.627 & 10.4 & 10.5 & 10.4 & 9.5 & 9.3 & 9.9 & 9.3 & 10.4 & 10 & 10.2 & 10.7 & 94.0 \\
\hline SEPFLOW & GAS & FQl-633 & 8699760 & 8944536 & 918579 & 9433790 & 9674541 & 991334 & 5043271 & 406140 & 655255 & 8960337 & & \\
\hline GAS TO GE-1-N & \#/HR & FR-607 & 496.2 & $\begin{array}{r}542.8 \\
\end{array}$ & 547 & \begin{tabular}{|l}
569.6 \\
\end{tabular} & \begin{tabular}{|r|}
570.7 \\
\end{tabular} & 576.8 & $\begin{array}{r}491.3 \\
\end{array}$ & $\begin{array}{r}530.5 \\
\end{array}$ & \begin{tabular}{|l|}
525.3 \\
\end{tabular} & 543.9 & 508.9 & 557.3 \\
\hline \multirow{4}{*}{$V \cdot 1-E$} & INCHES & LG.105 & 21.75 & 22.5 & 27 & 21.5 & 21.5 & 21.25 & 21 & 20.5 & 20.25 & 20 & 21 & 195 \\
\hline & & & & & & & & & & & & & & \\
\hline & & & & & & & & & & & & & & \\
\hline & & & & & & & & & & & & & & \\
\hline & & & & & & & & & & & & & & \\
\hline \multicolumn{15}{|l|}{ REMARKS } \\
\hline & & & & & & & & & & & & & & \\
\hline & & & & & & & & & & & & & & \\
\hline & & & & & & & & & & & & & & \\
\hline
\end{tabular}


May 14-16, 1990

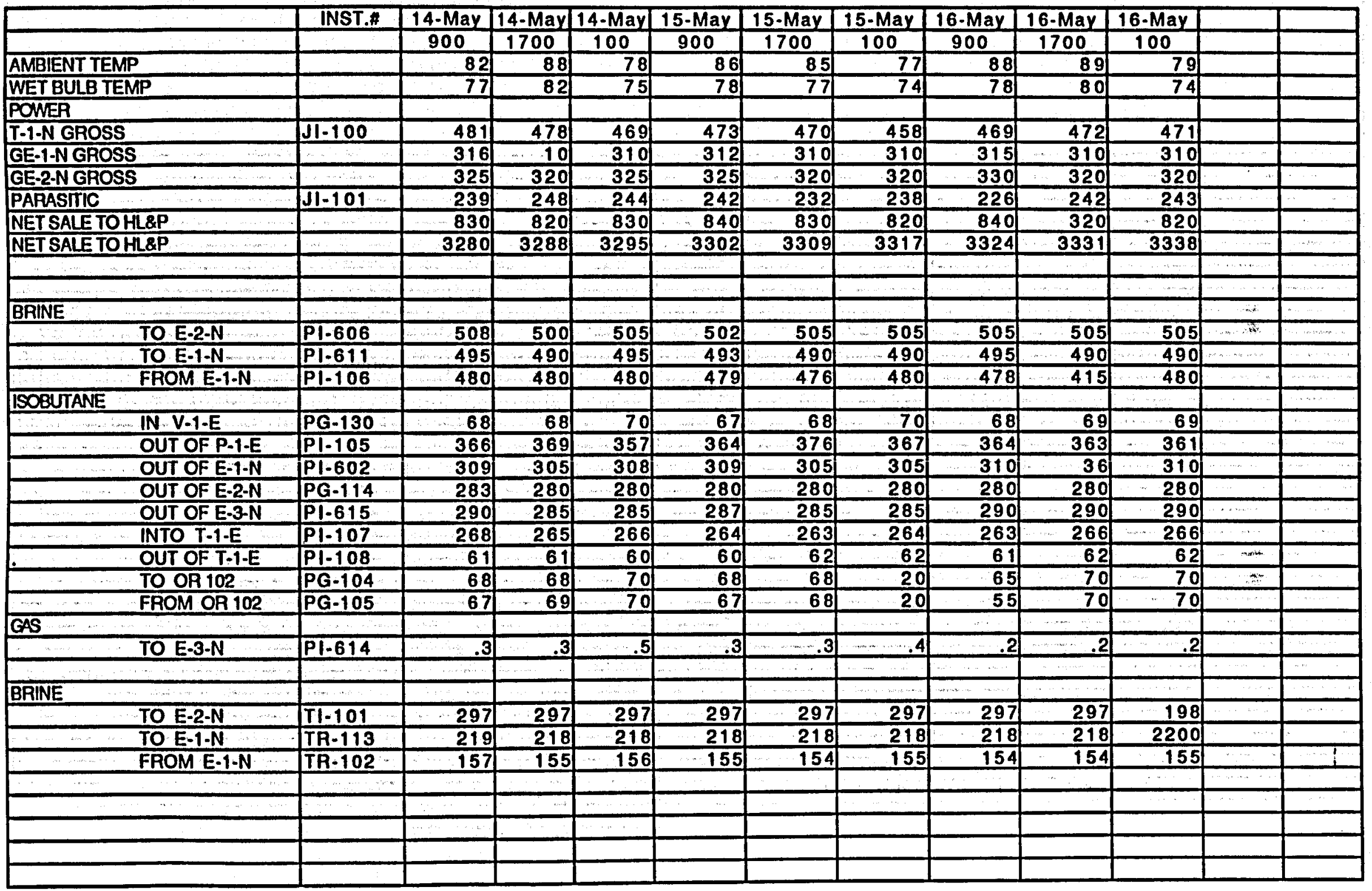


May 14.16, 1990

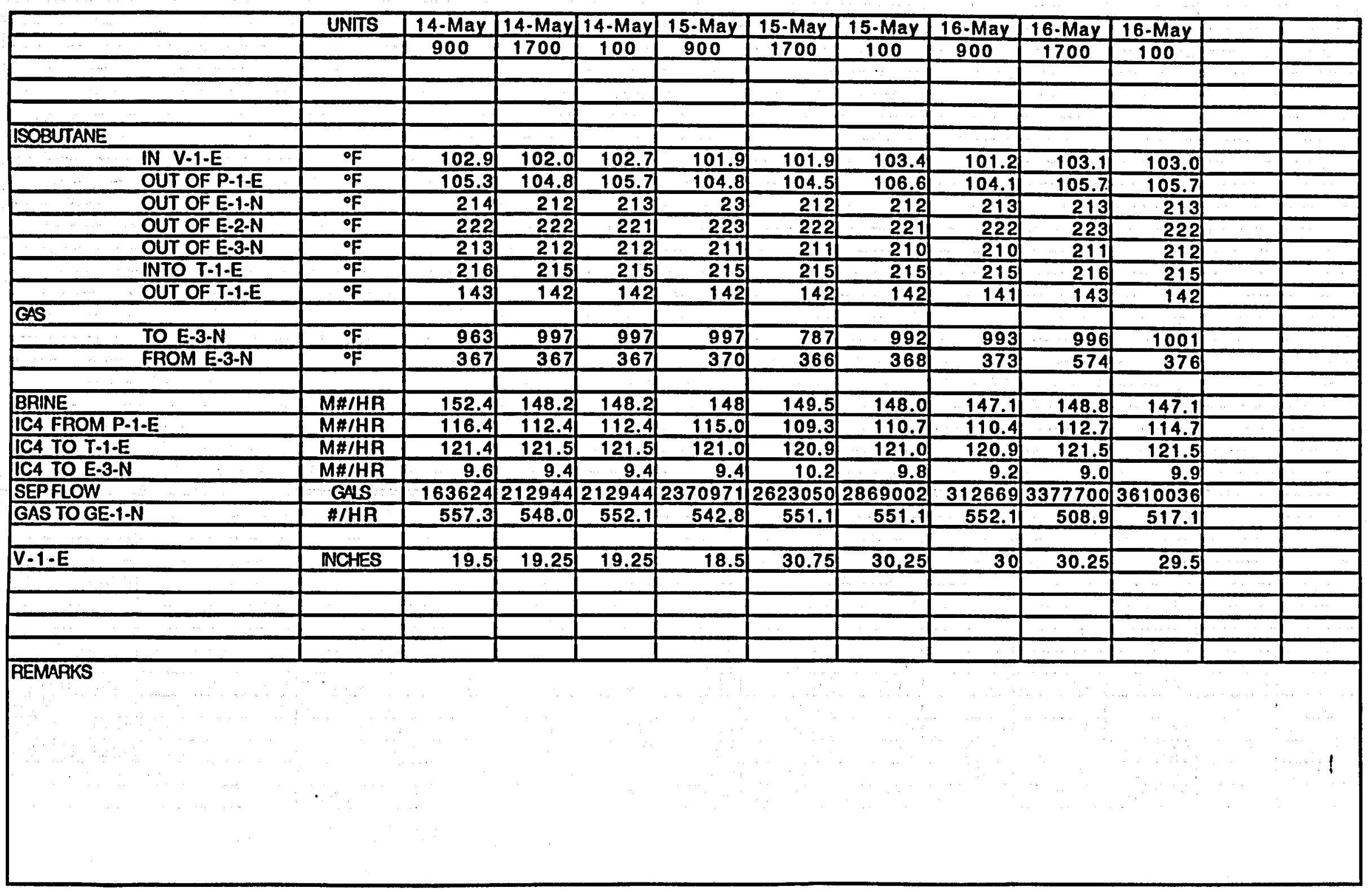


May $17-23,1990$

\begin{tabular}{|c|c|c|c|c|c|c|c|c|c|c|c|c|c|c|}
\hline & UNITS & INST.\# & 17-May & 17-May & 17-May & 18-May & 18-May & 18-May & 19-May & 19-May & 19-May & 20-May & 20.May & 20-May \\
\hline & & & 900 & 1700 & 100 & 900 & 1700 & 100 & 900 & 1700 & 100 & 900 & 1700 & 100 \\
\hline AMBIENT TEMP & ${ }^{\circ} \mathrm{F}$ & & 81 & 83 & 77 & 80 & 87 & 77 & 82 & 90 & 78 & 86 & 87 & 79 \\
\hline WET BULB TEMP & of & & 76) & 77 & 74 & 75 & 79 & 74 & 76 & 81 & 75 & 78 & 80 & 75 \\
\hline \multicolumn{15}{|l|}{ POWEA } \\
\hline T-1-N GROSS & KW & $J 1.100$ & 473 & 462 & 465 & 468 & 463 & 473 & 470 & 475 & 470 & 472 & 464 & 471 \\
\hline GE-1-NGROSS & KW & & 310 & 315 & 315 & 320 & 315 & 320 & 310 & 310 & 310 & 315 & 315 & 320 \\
\hline GE-2-NGROSS & $\overline{K W}$ & & 320 & 325 & 325 & 320 & 325 & 335 & 330 & 320 & 320 & 325 & 325 & 330 \\
\hline PARASITIC & $\mathbf{K W}$ & $J 1-101$ & 243 & 242 & 241 & 233 & 244 & 244 & 242 & 232 & 244 & 244 & 242 & 233 \\
\hline NET SALE TOHL\&P & KW & & 820 & 830 & 830 & 840 & 815 & 840 & 840 & 820 & 830 & 830 & 820 & 825 \\
\hline \multirow{3}{*}{ NET SALE TOHLAP } & $F D G$ & & 3346 & 3353 & 3361 & 3368 & 3374 & 3382 & 3390 & 3397 & 3404 & 3411 & 3419 & 3427 \\
\hline & $\mathrm{KW} \cdot \mathrm{HA}$ & & & & & & & & & & & & & \\
\hline & & & & & & & & & & & & & & \\
\hline \multicolumn{15}{|l|}{ BAINE. } \\
\hline TO E-2-N & PSIG & $P 1.606$ & 5.10 & 502 & 505 & 505 & 502 & 504 & 510 & 505 & 505 & 505 & 502 & 505 \\
\hline TO E-1.N & PSTQ & $P 1.611$ & 500 & & 490 & 485 & 480 & 482 & 485 & 480 & 485 & 480 & 478 & 485 \\
\hline FROM E-1-N & PSTG & $P 1.106$ & 485 & 477 & 480 & 485 & 477 & 480 & 476 & 475 & 478 & 477 & 473 & 476 \\
\hline \multicolumn{15}{|l|}{ BSOBUTANE } \\
\hline IN V.1.E & PST & PG-130 & 70 & 69 & 68 & 68 & 69 & 68 & 68 & 69 & 68 & $\begin{array}{r}468 \\
\end{array}$ & 69 & 69 \\
\hline OUT OF P-1-E & PSIG & PI-105 & 360 & 355 & 365 & 358 & 357 & 359 & 363 & 356 & 361 & 356 & 351 & 358 \\
\hline OUT OF E-1-N & PS1G & $\mathrm{PI}-602$ & 310 & 305 & 310 & 305 & 306 & 306 & 305 & 305 & 305 & 310 & 306 & 310 \\
\hline OUT OF E-2-N & PSG & $P G-114$ & 275 & 278 & 280 & 275 & 276 & 278 & 279 & 280 & 280 & 280 & 279 & 280 \\
\hline OUT OF E-3-N & PSIG & $P \mid .615$ & 285 & 278 & 290 & 280 & 277 & 278 & 279 & 280 & 285 & 282 & 280 & 285 \\
\hline INTO T-1-E & PSIG & PI.107 & 269 & 263 & 264 & 263 & 264 & 264 & 264 & 266 & 266 & 265 & 265 & 266 \\
\hline OUT OF T-1.E & PSIG & P1.108 & 67 & .62 & 64 & 61 & 62 & 60 & 77 & 78 & 79 & 79 & 80 & 80 \\
\hline TO OA 102 & PSTG & PG-104 & 70 & 69 & 70 & 70 & 69 & 69 & 68 & 69 & 70 & 68 & 70 & 70 \\
\hline FROM OA 102 & PSIG & PQ-105 & 70 & 69 & 70 & 69 & 69 & 69 & 68 & 69 & 70 & 68 & -270 & 70 \\
\hline \multicolumn{15}{|l|}{ GS } \\
\hline TOE-3-N & PSTG & $P \mid-614$ & .2 & .2 & .2 & .3 & .2 & .2 & .2 & .2 & .2 & .2 & .2 & .2 \\
\hline & & & & & & & & & & & & & & \\
\hline \multicolumn{15}{|l|}{ BRINE } \\
\hline TO E-2-N & ${ }^{\circ} \mathrm{F}$ & $T I .101$ & 298 & 298 & 298 & 297 & 297 & 297 & 297 & 297 & 297 & 297 & 297 & 297 \\
\hline TO E-1-N & OF & TR-113 & 217 & 217 & 219 & 217 & 218 & 218 & 218 & 218 & 218 & 219 & 218 & 219 \\
\hline FROM E-1-N & of & TR-102 & 154 & 155 & 155 & 155 & 156 & 155 & 155 & 155 & 156 & 155 & 156 & 156 \\
\hline & & & & & & & & & & & & & & \\
\hline & & & & & & & & & & & & & & \\
\hline & & & & & & & & & & & & & & \\
\hline & & & & & & & & & & & & & & \\
\hline & & & & & & & & & & & & & & \\
\hline
\end{tabular}


GEOPRESSURED HYBAID POWER SYSTEM - MANAGEMENT REPOAT DATA

May 17-23, 1990

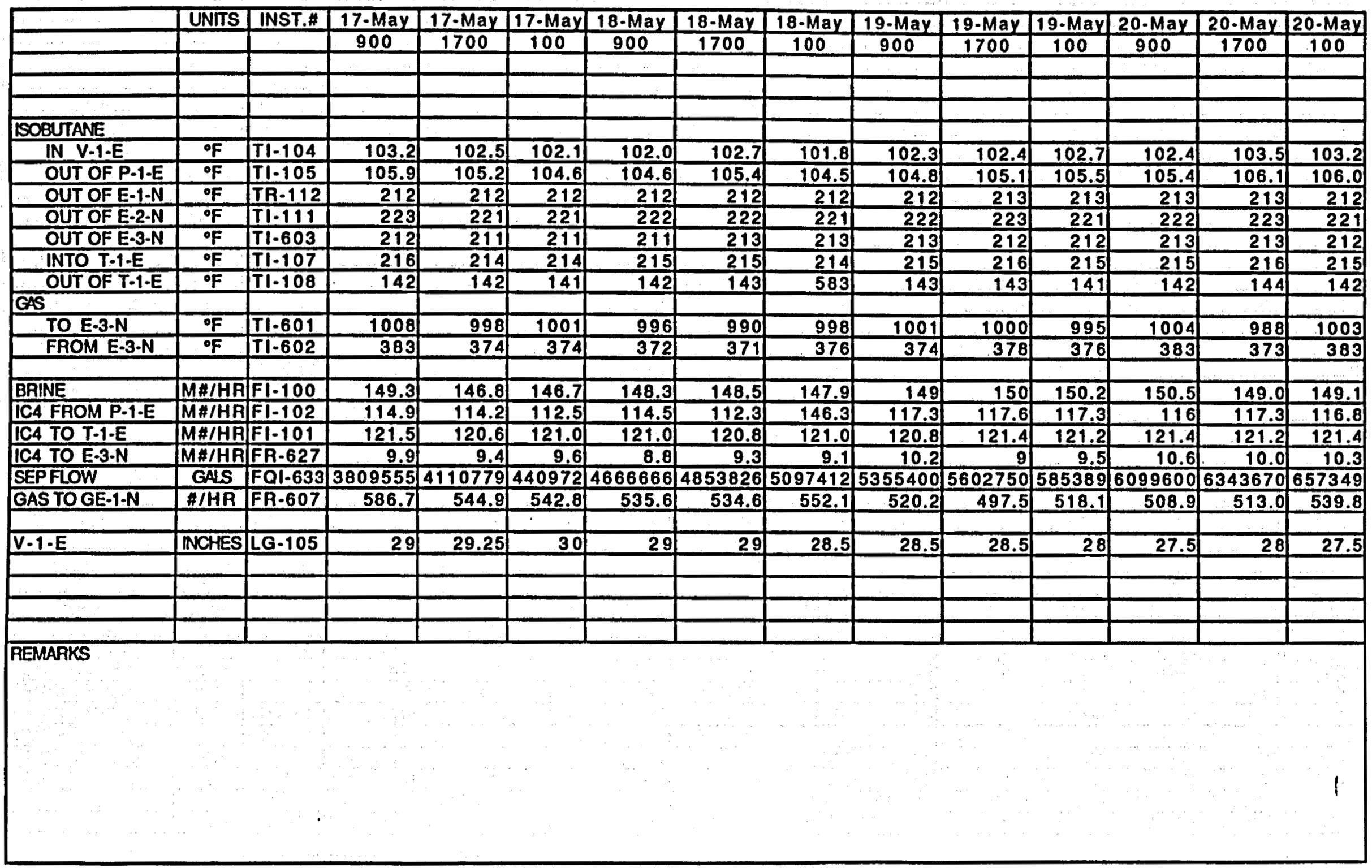


May 21-23, 1990

\begin{tabular}{|c|c|c|c|c|c|c|c|c|c|c|c|c|}
\hline & INST.\# & 21-May & 21-May & 21-May & 22-May & 22-May & 22-May & 23-May & 23-May & 23-May & & \\
\hline & & 900 & 1700 & 100 & 900 & 1700 & 100 & 900 & 1700 & 100 & & \\
\hline AMBIENT TEMP & & 86 & 93 & 78 & 92 & 70 & 72 & 73 & 85 & 74 & & \\
\hline WET BULB TEMP & & 79 & 82 & 75 & 80 & 69 & 68 & 67 & 75 & 70 & & \\
\hline POWER & & & & & & & & & & & & \\
\hline T-1-N GROSS & J1-100 & 470 & 455 & 467 & 457 & 485 & 482 & 497 & 488 & 483 & & \\
\hline GE-1-NGROSS & & 310 & 312 & 315 & 300 & 325 & 300 & 0 & 0 & 0 & & \\
\hline GE-2-NGROSS & & 320 & 315 & 325 & 330 & 340 & 300 & 345 & 340 & 340 & & \\
\hline PARASITIC & $\mathrm{J1}-101$ & 242 & 242 & 244 & 241 & 245 & 243 & 243 & 242 & 243 & & \\
\hline NET SALE TOHL\&P & & 820 & 790 & 810 & 800 & 860 & 800 & 595 & 575 & 570 & & \\
\hline NET SALE TO HL\&P & & 3433 & 3940 & 3448 & 3454 & 3460 & 3468 & 3474 & 3479 & 3484 & & \\
\hline & & & & & & & & & & & & \\
\hline & & & & & & & & & & & & \\
\hline BAINE & & & & & & & & & & & & \\
\hline TO E-2-N & $\mathrm{PI} .606$ & 505 & 502 & 510 & 503 & 509 & 505 & 504 & 505 & 505 & & \\
\hline TO E-1-N & $\mathrm{P} \mid-611$ & 485 & 478 & 485 & 482 & 488 & 485 & 479 & 478 & 480 & & \\
\hline FROM E-1.N & $P \mid-106$ & 480 & 475 & 477 & 478 & 480 & 480 & 476 & 473 & 480 & & \\
\hline ISOBUTANE & & & & & & & & & & & & \\
\hline IN V.1-E & $P G=130$ & 70 & 71 & 69 & 69 & 64 & 66 & 64 & 67 & 68 & & \\
\hline OUT OF P.1.E & $\mathrm{PI}-105$ & 356 & 355 & 363 & 371 & 361 & 364 & 359 & 351 & 356 & & \\
\hline OUT OF E-1-N & $P !-602$ & 305 & 305 & 310 & 310 & 277 & 305 & 310 & 317 & 310 & & \\
\hline OUT OF E-2-N & $\overline{P G-114}$ & 280 & 269 & 280 & 280 & 305 & 275 & 278 & 280 & 280 & & \\
\hline OUT OF E-3-N & PI.615 & 280 & 278 & 285 & 285 & 278 & 280 & 278 & 283 & 280 & & \\
\hline INTO T-1.E & $\mathrm{P} \mid \cdot 107$ & 266 & 266 & 265 & 264 & 265 & 263 & 264 & 267 & 266 & & \\
\hline OUT OF T-1-E & $\mathrm{PI}-108$ & 81 & 78 & 77 & 82 & 72 & 75 & 75 & 77 & 77 & & \\
\hline TO OR 102 & PG-104 & 69 & 70 & 70 & 69 & 62 & 65 & 61 & 65 & 65 & & \\
\hline FAOM OA 102 & PG.105 & 65 & 70 & 70 & 69 & 61 & 60 & 60 & 65 & 62 & 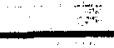 & \\
\hline GAS & & & & & & & & & & & & \\
\hline TO E-3-N & $\mathrm{Pl} .614$ & .2 & .2 & .2 & .2 & .2 & .2 & .2 & .2 & .2 & & \\
\hline & 5 & & & & & & & & & & & \\
\hline BRINE & & & & & & & & & & & & \\
\hline TO E-2-N & $\mathrm{TI}-101$ & 297 & 298 & 298 & 298 & 297 & 298 & 297 & 298 & 298 & & \\
\hline TO E-1-N & TR-113 & 219 & 217 & 218 & 218 & 218 & 218 & 220 & 219 & 218 & & \\
\hline FROM E-1-N & TR-102 & 155 & 156 & 154 & 155 & 154 & 154 & 155 & 157 & 157 & & \\
\hline & & & & & & & & & & & & \\
\hline & & & & & & & & & & & & \\
\hline & & & & & & & & & & & & \\
\hline & & & & & & & & & & & & \\
\hline
\end{tabular}


May 21-23, 1990

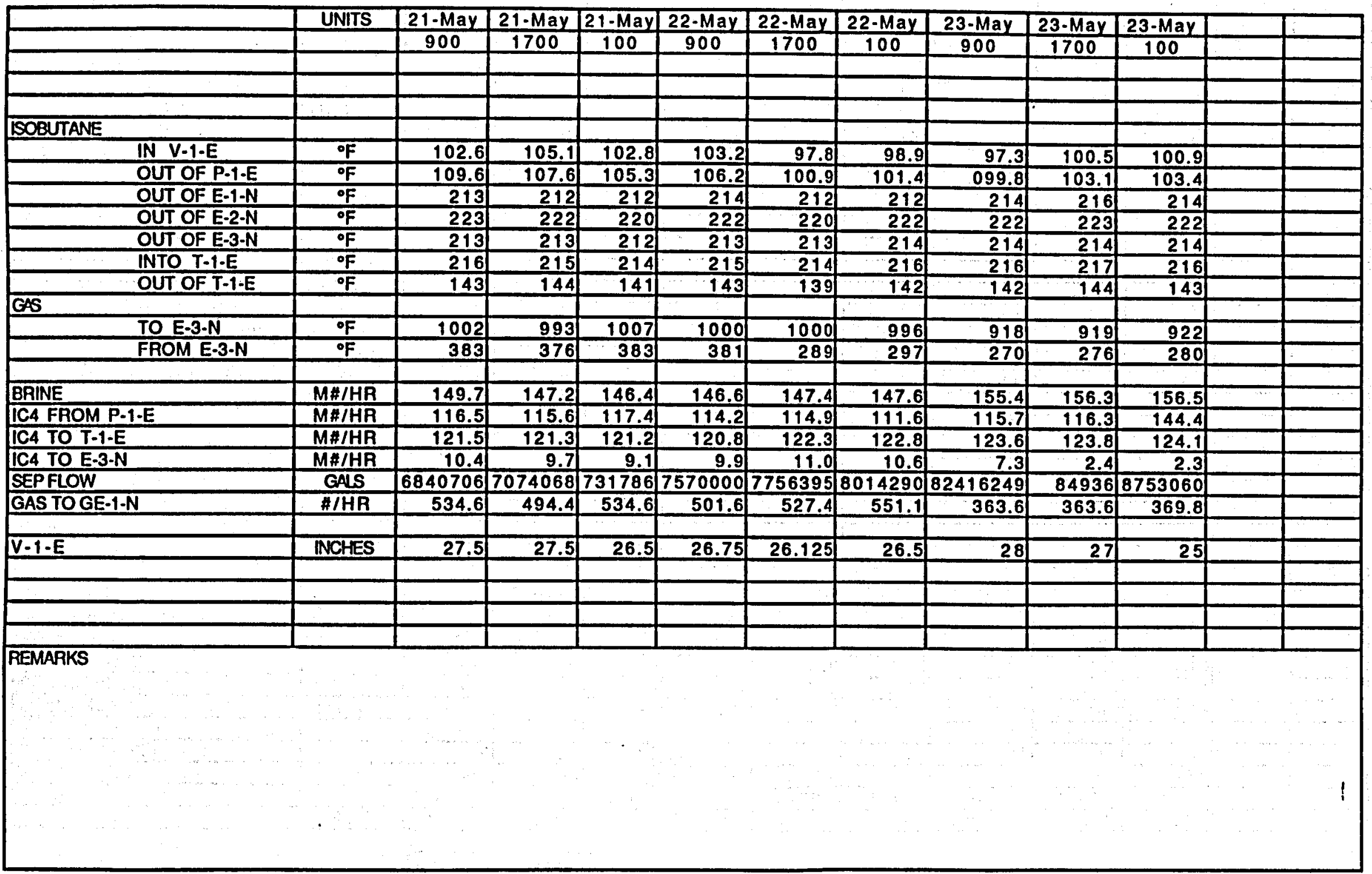


May 24-27, 1990

\begin{tabular}{|c|c|c|c|c|c|c|c|c|c|c|c|c|c|c|}
\hline & UNITS & INST.\# & 24-May & 24-May & 24-May & 25-May & 25-May & 25.May & 26-May & 26-Mays: & 26-May & 27-May & 27-May & 27-May \\
\hline & & & 900 & 1700 & 100 & 900 & 1700 & 100 & 900 & \begin{tabular}{|l|}
1700 \\
\end{tabular} & 100 & 900 & 1700 & 100 \\
\hline AMBIENT TEMP & ${ }^{\circ} \mathrm{F}$ & & 80 & 90 & 75 & 91 & 90 & 76 & 92 & 93 & 77 & 80 & 99 & 79 \\
\hline WET BULB TEMP & oF & & 72 & 82 & 73 & 79 & 80 & 72 & 811 & 89 & 74 & 80 & 89 & 75 \\
\hline \multicolumn{15}{|l|}{ POWER } \\
\hline T-1.NGROSS & KW & J1.100 & 474 & 465 & 480 & 465 & 470 & 482 & 474 & 461 & 0 & 0 & 0 & 0 \\
\hline GE-1.NGROSS & $\overline{K W}$ & & 0 & 225 & 330 & 330 & 330 & 330 & 330 & 330 & 330 & 335 & 330 & 330 \\
\hline GE-2-NGROSS & KW & & 340 & 325 & 330 & 335 & 330 & 330 & 330 & 330 & 330 & 330 & 330 & 330 \\
\hline PARASITIC & $\mathbf{K W}$ & J1-101 & 242 & 230 & 244 & 244 & 243 & 244 & 243 & 241 & 16 & 23 & 24 & 24 \\
\hline NET SALE TO HLAP & $\mathrm{KW}$ & & 565 & 840 & 860 & 845 & 840 & 860 & 855 & 830 & 580 & 275 & 570 & 580 \\
\hline \multirow{2}{*}{ NET SALE TO HLQP } & $F D G$ & & 3489 & 3495 & 3502 & 3511 & 3517 & 3525 & 3532 & 3539 & 3545 & 3550 & 3554 & 3558 \\
\hline & KW-HA & & & & & & & & & & & & & \\
\hline & & \\
\hline BRINE & & & & & & & & & & & & & & \\
\hline TO E-2-N & PSIG & $\mathrm{P} 1.606$ & 502 & 505 & 510 & 502 & 505 & 505 & 503 & 504 & 0 & 0 & 0 & 0 \\
\hline TO E.1.N & PSIG & PI.611 & 480 & 480 & 485 & 482 & 480 & 485 & 482 & 480 & 0 & 0 & 0 & $\underline{0}$ \\
\hline FROM E-1-N & PSIG & $\mathrm{Pl}-106$ & 476 & 480 & 480 & 478 & 480 & 478 & 475 & 474 & 5 & 2 & 1 & 0 \\
\hline \multicolumn{15}{|l|}{ SOBSUTANE } \\
\hline IN V.1.E & PSIG & $P G-130$ & 70 & 70 & 69 & 69 & 69 & 68 & 69 & 72 & 49 & 59 & 68 & 60 \\
\hline OUT OF P.1-E & PSTG & $P 1.105$ & 359 & 367 & 354 & 361 & 356 & 354 & 362 & 347 & 48 & 60 & 69 & 58 \\
\hline OUT OF E-1-N & PSIG & P1-602 & 311 & 305 & 315 & 313 & 315 & 315 & 309 & 311 & 50 & 59 & 30 & 0 \\
\hline OUT OF E-2-N & PSIG & PG-114 & 281 & 275 & 280 & 280 & 280 & 280 & 280 & 280 & 50 & 39 & 30 & 0 \\
\hline OUT OF E-3-N & PSTG & $\mathrm{P} 1.615$ & 281 & 280 & 280 & 278 & 280 & 280 & 280 & 280 & 50 & 57 & 65 & 55 \\
\hline INTO T-1-E & PSTG & $\mathrm{P} \mid \cdot 107$ & 267 & 263 & 267 & 263 & 264 & 266 & 265 & 267 & 49 & 40 & 44 & 37 \\
\hline OUT OF T-1-E & PSIG & $\mathrm{PI}-108$ & 80 & .79 & 77 & 81 & 78 & 77 & 82 & 82 & 49 & 41 & 41 & 36 \\
\hline TO OA 102 & PSTG & PG-104 & 68 & 65 & 65 & 68 & 70 & 70 & 68 & 72 & 50 & 0 & 70 & 60 \\
\hline FROM OR 102 & PSIG & PG-105 & 67 & 70 & 70 & 65 & 65 & 70 & 64 & 72 & 50 & 의 & 6,5 & 55 \\
\hline \multicolumn{15}{|l|}{ GAS } \\
\hline TO E-3-N & PSIG & PI-614 & .0 & .2 & .2 & .2 & .2 & .2 & .2 & .2 & .0 & .0 & .0 & .0 \\
\hline \multirow{2}{*}{\multicolumn{15}{|c|}{ BRINE }} \\
\hline & & & & & & & & & & & & & & \\
\hline TO E-2-N & of & $T 1.101$ & 298 & 297 & 298 & 298 & 297 & 298 & 298 & 297 & 249 & 244 & 239 & 223 \\
\hline TO E-1.N & ${ }^{\circ} \mathrm{F}$ & $T \mathrm{TR}-113$ & 219 & 218 & 219 & 217 & 218 & 218 & 218 & 219 & 236 & 228 & 222 & 166 \\
\hline FROM E-1-N & ${ }^{\circ} \mathrm{F}$ & TR-102 & 157 & 154 & 155 & 154 & 153 & 152 & 154 & 153 & 112 & 203 & 199 & 149 \\
\hline & & & & & & & & & & & & & & \\
\hline & & & & & & & & & & & & & & \\
\hline & & & & & & & & & & & & & & \\
\hline & & & & & & & & & & & & & & \\
\hline & & & & & & & & & & & & & & \\
\hline
\end{tabular}




\section{GEOPRESSURED HYBRID POWER SYSTEM - MANAGEMENT REPORT DATA}

May 24-27, 1990

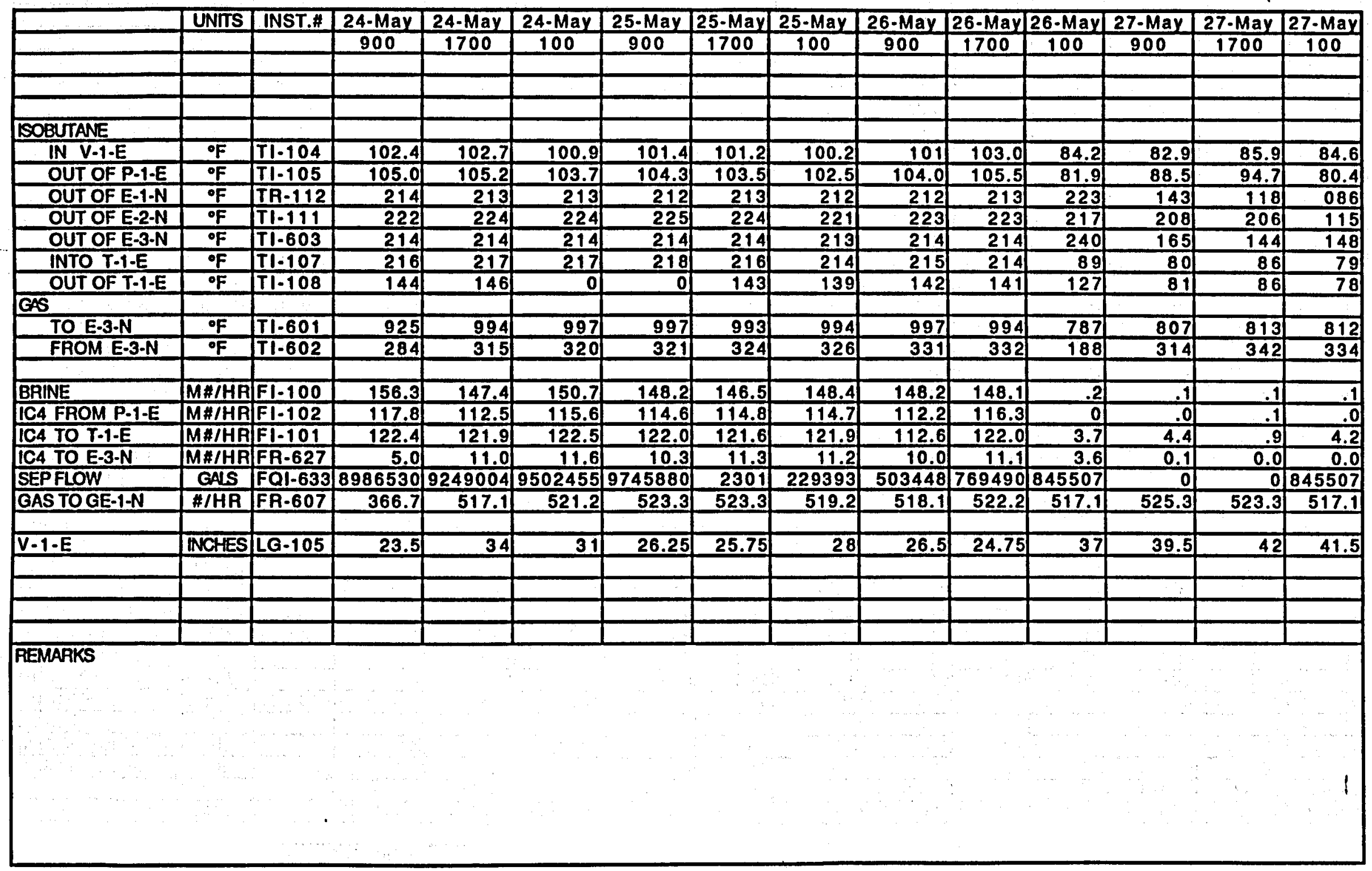


May 28-30, 1990

\begin{tabular}{|c|c|c|c|c|c|c|c|c|c|c|c|c|}
\hline & INST.\# & 28-May & 28-May & 28-May & 29-May & 29-May & 29.May & 30-MaY & 30-May & 30-May & & \\
\hline & & 900 & 1700 & 100 & 900 & 1700 & 100 & 900 & 1700 & 100 & & \\
\hline AMBIENT TEMP & & 86 & 95 & 75 & 80 & 85 & 78 & & & & & \\
\hline WET BULB TEMP. & & 78 & 86 & 72 & 75 & 78 & 74 & & & & & \\
\hline POWEA & & & & & & & & & & & & \\
\hline T-1-N GROSS & J1.100 & 0 & 아 & 0 & 0 & o & of & & & & & \\
\hline GE-1-NGROSS & & 335 & 320 & 320 & 335 & 335 & 330 & & & & & \\
\hline GE-2-N GROSS & & 335 & 330 & 320 & 335 & 335 & 330 & & & & & \\
\hline PARASITIC & $J 1-101$ & 34 & 19 & 19 & 6 & 29 & 29 & & & & & \\
\hline NET SALE TO HL\&P & & 565 & 550 & 575 & 610 & 580 & 590 & & & & & \\
\hline NET SALETOHL\&P & & 3565 & 3570 & 3574 & 3580 & 3585 & 3589 & & & & & \\
\hline & & & & & & & & & & & & \\
\hline & & & & & & & & & & & & \\
\hline BRINE & & & & & & $\therefore$ & & & & & & \\
\hline TO E-2-N & PI-606 & 0 & (0) & 525 & 530 & (0) & 이 & & & & & \\
\hline TO E-1-N & PI.611 & 0 & of & 525 & 530 & 0 & 0 & & & & 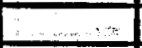 & \\
\hline FROM E-1-N & PI-106 & 0 & 0) & 529 & 529 & 17 & 535 & & & & & \\
\hline SOBUTANE & 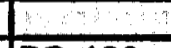 & & & & $\therefore$ & 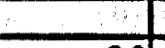 & & & & & & \\
\hline IN $V-1-E$ & $P G-130$ & 57 & 63 & 54 & 47 & 60 & 60 & & & & & \\
\hline OUT OF P-1.E & $P /-105$ & 56 & 61 & 53 & 47 & 62 & 60 & & & & & \\
\hline OUT OF E-1-N & Pl-602 & 0 & 0 & 40 & 40 & 60 & 0 & & & & & \\
\hline OUT OF E-2-N & PG.114 & 0 & 0 & 40 & 40 & 45 & o & & & & & \\
\hline OUT OF E-3-N & $\mathrm{P} \mid-615$ & 59 & 60 & 60 & 50 & 0 & 50 & & & & & \\
\hline INTO T-1-E & $P \mid-107$ & 36 & 44 & 21 & 45 & 45 & 25 & & & & & \\
\hline OUT OF T-1-E & $P \mid-108$ & 37 & 38 & 21 & 44 & 41 & 23 & & & & & \\
\hline TO OR 102 & PG.104 & 54 & 60 & 60 & 50 & 60 & 55 & & & & $\therefore$ & \\
\hline FROM OR 102 & PG.105 & 57 & 55 & 55 & 50 & 60 & 55 & & & & & \\
\hline GAS & & & & & & & & & & & & \\
\hline TO E-3-N & $\mathrm{P} / \mathrm{-614}$ & .0 & .0 & .0 & .0 & .0 & .0 & & & & & \\
\hline & & & & & & & & & & & & \\
\hline BRINE & & & & & & & & & & & & \\
\hline TO E-2-N & Tl.101 & 208 & 118 & 222 & 206 & 274 & 270 & & & & & \\
\hline TOE-1-N & TR-113 & 124 & 97 & 86 & 85 & 97 & 103 & & & & & \\
\hline FROM E-1-N & TR-102 & 96 & 113 & 102 & 92 & 94 & 92 & & & & & \\
\hline & & & & & & & & & & & & \\
\hline & & & & & & & & & & & & \\
\hline & & & & & & & & & & & & \\
\hline & & & & & & & & & & & & \\
\hline
\end{tabular}


May $28-30,1990$

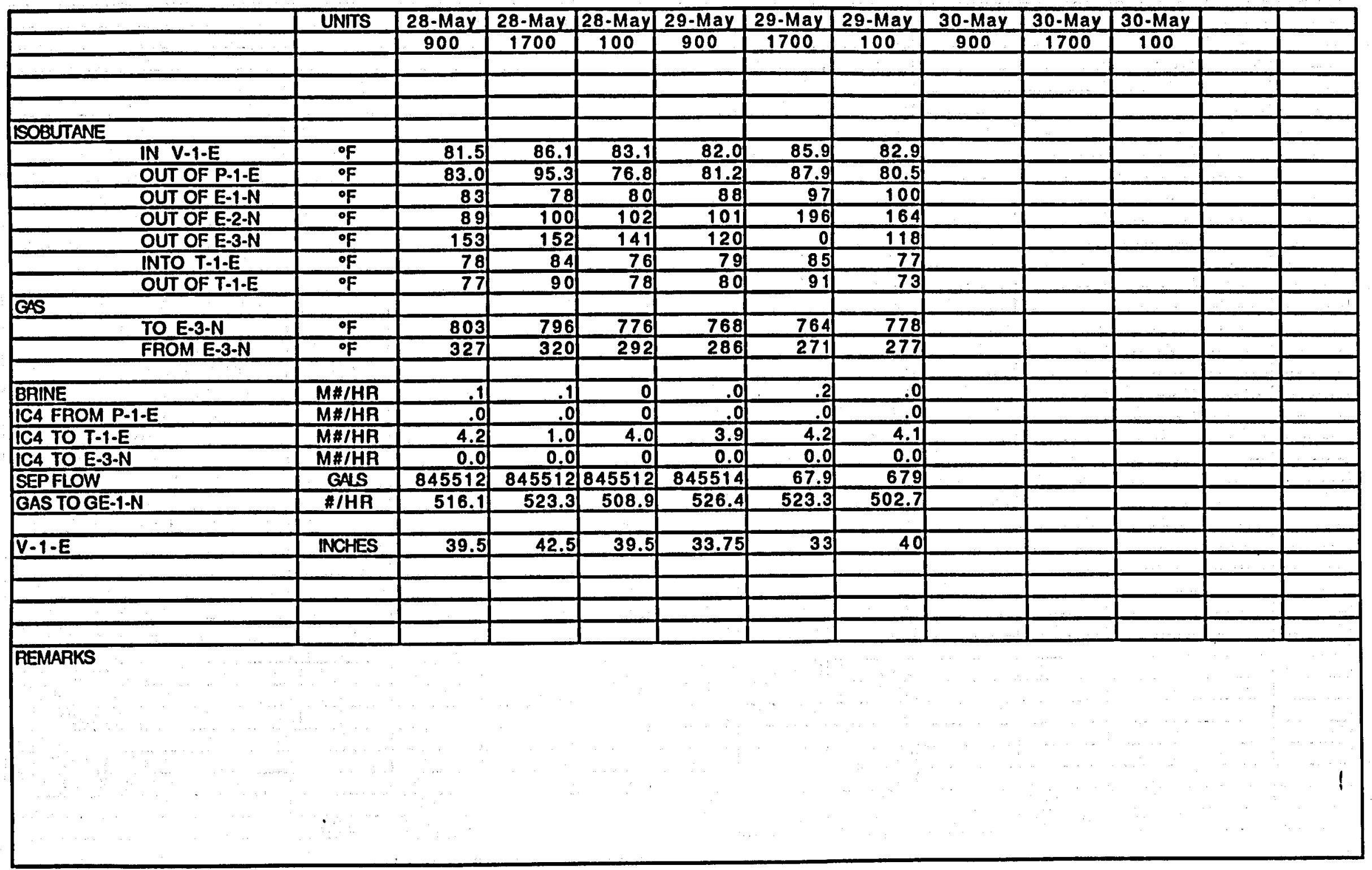

